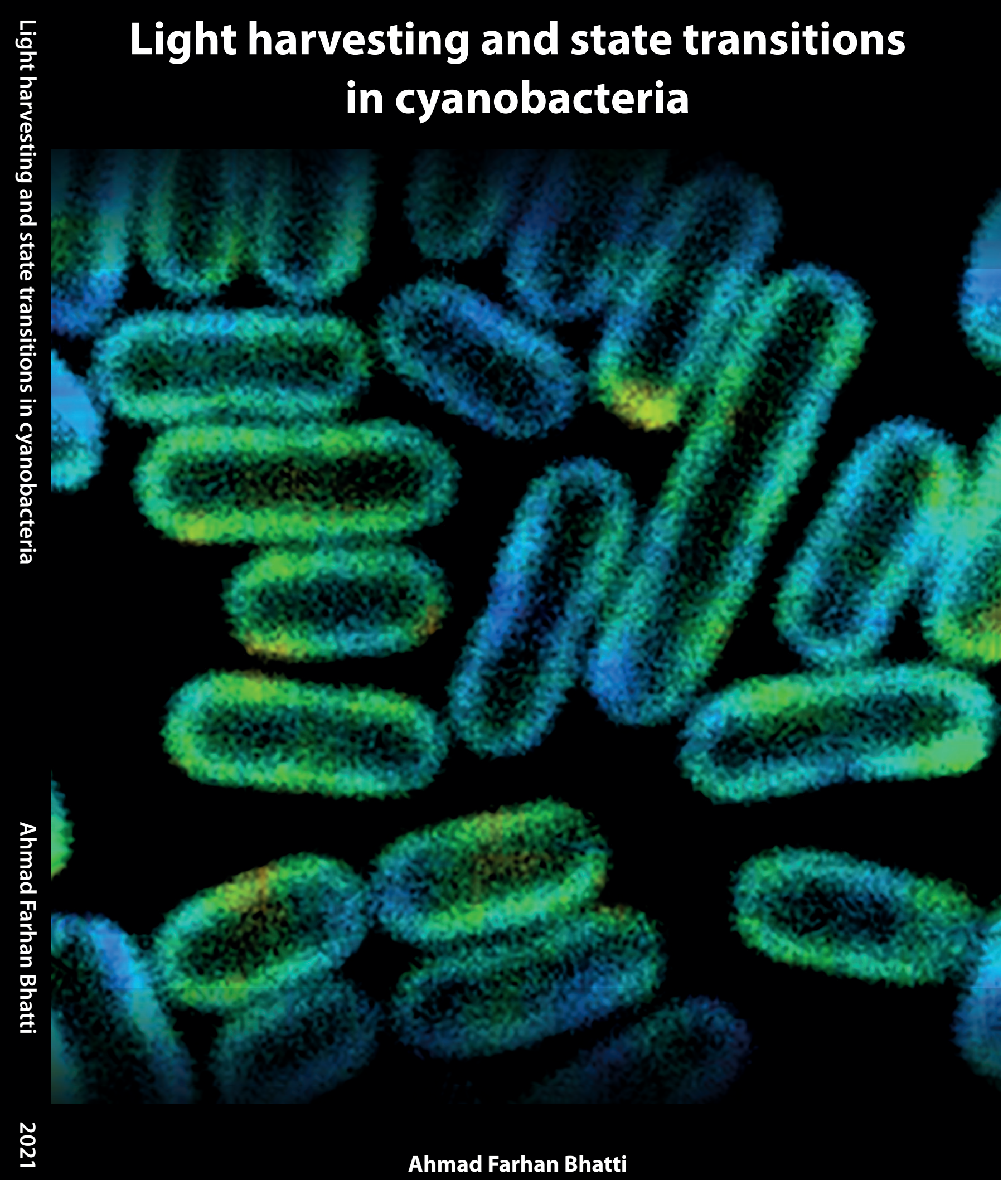




\section{Propositions}

1. The mechanisms of state transitions and OCP-induced non-photochemical quenching in cyanobacteria interfere with each other.

(this thesis)

2. The role of the thylakoid membrane in the regulation of light harvesting is underexplored in cyanobacteria.

(this thesis)

3. Progress in science and technology has increased the life expectancy of human individuals, whereas it has decreased the expectancy of human life on Earth.

4. Many scientific achievements have fixed the focus of mankind on exploration of the universe around, while an equally great and complex universe within ourselves remains underexplored.

5. Modern technologies of communication, media and virtual reality provide so many ways of distraction that they pose a significant challenge to the quality of real life.

6. Pursuing incremental progress in a very specialized research topic may come at the cost of the personal growth of a researcher.

7. The variety of available food at the cafeterias of our university does not reflect the amazing international diversity this institute offers.

Propositions belonging to the thesis, entitled

Light harvesting and state transitions in cyanobacteria

Ahmad Farhan Bhatti

Wageningen, 19 November 2021 


\section{Light harvesting and state transitions in cyanobacteria}

Ahmad Farhan Bhatti 


\section{Thesis committee}

\section{Promotor}

Prof. Dr Herbert van Amerongen

Professor of Biophysics

Wageningen University \& Research

\section{Co-promotor}

Dr Emilie Wientjes

Assistant professor, Laboratory of Biophysics

Wageningen University \& Research

\section{Other members}

Prof. Dr Paul C. Struik, Wageningen University \& Research

Prof. Dr Conrad Mullineaux, Queen Mary University of London, UK

Dr Volha U. Chukhutsina, Imperial College London, UK

Dr Nicoletta Liguori, VU, Amsterdam

This research was conducted under the auspices of the Graduate School EPS (Experimental Plant Sciences). 


\title{
Light harvesting and state transitions in cyanobacteria
}

\author{
Ahmad Farhan Bhatti
}

Thesis

submitted in fulfilment of the requirement for the degree of doctor at Wageningen University

by the authority of the Rector Magnificus,

Prof. Dr A.P.J. Mol,

in the presence of the

Thesis Committee appointed by the Academic Board

to be defended in public

on Friday 19 November 2021

at 4 p.m. in the Aula. 
Ahmad Farhan Bhatti

Light harvesting and state transitions in cyanobacteria 162 pages.

PhD thesis, Wageningen University, Wageningen, the Netherlands (2021)

ISBN: $\quad 978-94-6395-292-7$

DOI: https://doi.org/10.18174/554555 
Content

$\begin{array}{lll}\text { Chapter } 1 & \text { General introduction } & 7\end{array}$

Chapter 2 State transitions in cyanobacteria studied with picosecond 33 fluorescence at room temperature

Chapter 3 Structural changes in PBS-PSIl complexes modulate the excited-state 59 dynamics of PSII during state transitions in cyanobacteria

Chapter 4 State transitions and photosystems spatially resolved in individual 79 cells of the cyanobacterium Synechococcus elongatus

Chapter 5 Far-red allophycocyanin increases both the absorption cross107 section and photochemical efficiency of chlorophyll f-containing photosystem II

Chapter 6 General Discussion 
CHAPTER

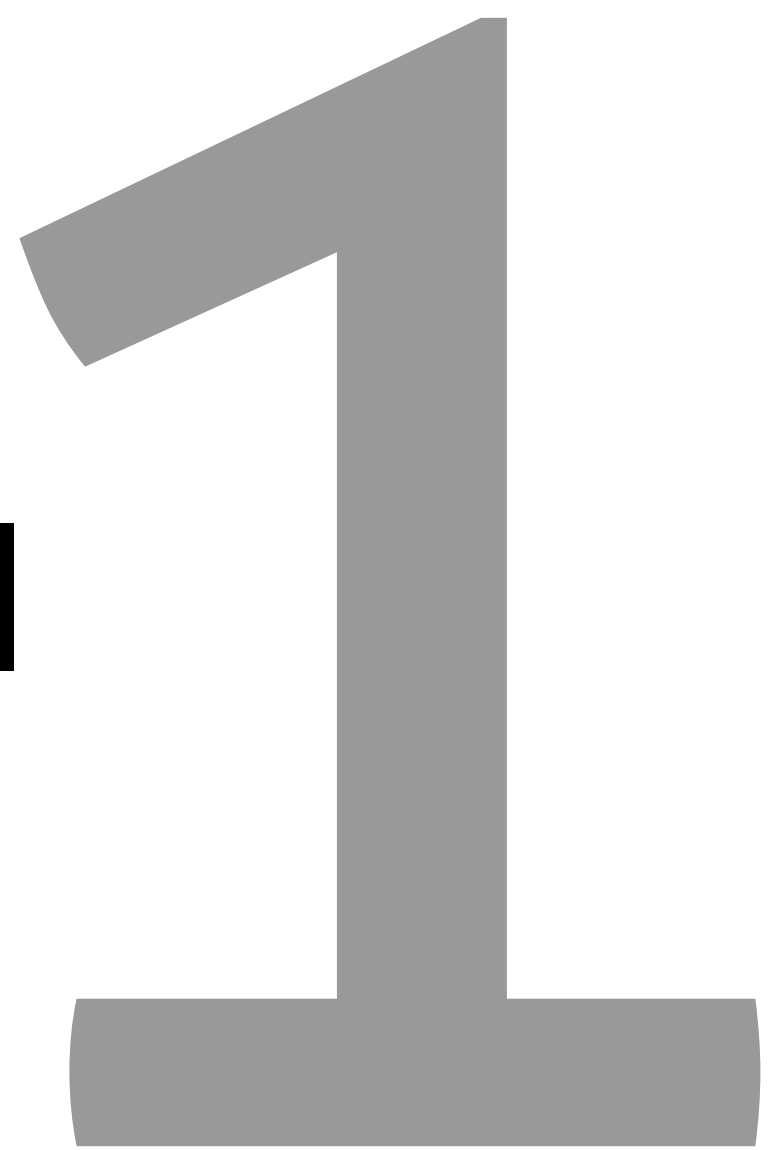


General introduction 



\section{Background and Scope}

Oxygenic photosynthesis is an abundant process on Earth, responsible for biological conversion of the free energy of sunlight into storable chemical energy of organic compounds ${ }^{[1]}$. While the origins of oxygenic photosynthesis still remain ill-determined $[2,3]$, the significance of this process for life in our biosphere cannot be overstated. Once responsible for setting the stage for the evolution of unicellular life to incredibly complex and diverse life forms, the process still remains the main source of its sustenance ${ }^{[4,5]}$. In fact, the amount of organic matter produced by photosynthesis sets an upper limit on the quantity of living organisms. In aquatic habitats the bulk of photosynthesis is carried out by cyanobacteria, algae and diatoms. In non-aquatic environments plants are the main organisms contributing to photosynthesis.

Solar radiation, which is a prerequisite for photosynthesis, under various circumstances also poses various challenges to the existence and efficiency of the photosynthetic organisms. These challenges appear due to changes in the quality and intensity of light reaching the photosynthetic apparatus ${ }^{[6,7]}$. In aquatic environments, light fluctuations are accentuated due to factors such as varying degree of water opacity, shade and depth. To meet with the challenge of a rapidly varying light environment, aquatic photosynthetic organisms have developed strategies allowing their photosynthetic apparatus to adjust to the light environment on the time scale of seconds to minutes ${ }^{[8]}$. These strategies can be broadly categorized into high-light and low-light acclimation responses. At high light intensities, organisms use non-photochemical quenching (NPQ) processes as photoprotection mechanisms at the levels of light harvesting and photosystems complexes ${ }^{[9-14]}$. State transitions form a low-light and light-quality acclimation response ${ }^{[15,16]}$, which is partly understood in plants ${ }^{[16,17]}$ and green algae ${ }^{[18-20]}$, but remains largely mysterious in cyanobacteria.

Chromatic adaptation is another strategy by means of which, photosynthetic organisms tailor the composition of the photosynthetic pigments in response to the dominant color of the ambient light ${ }^{[21-24]}$. Red and far-red photons can sometimes comprise a significant portion of the sunlight in microbial mats, aquatic habitats ${ }^{[25,26]}$ and in the shades of the canopy ${ }^{[26,27]}$, but the far-red photons are poorly absorbed by $\mathrm{Chl}$ a ${ }^{[28]}$, a pigment on which oxygenic photosynthesis mainly relies. The creative potential of light and photosynthetic organisms is manifested in an increasingly large group of cyanobacteria strains, where the long-term red-shift of the light environment induces the creation of a new photosynthetic apparatus (PSI, PSII, light-harvesting antenna), incorporating the red-light absorbing ChI $d$ and $\mathrm{Chl} f$ pigments ${ }^{[29-35]}$. Harvesting of sunlight $>700 \mathrm{~nm}$ also appears promising for the increased productivity of the crops and the biomass production. However, Mascoli et al ${ }^{[36]}$ have pointed out the fundamental challenge regarding the prospects of introducing 
far-red light absorbing pigments for increased biomass productivity by showing that the insertion of $\mathrm{Chl} f$ significantly increases light absorption but slows down primary photochemistry and subsequently the photosynthetic yield in cyanobacteria.

Two different acclimation strategies of cyanobacteria to the changes in light quality, i.e., state transitions and far-red light acclimation are studied in this work. The focus of this thesis is to investigate state transitions in cyanobacteria, with the purpose to elucidate the underlying mechanism. It encompasses the understanding of light harvesting under changing light conditions, interaction of phycobilisomes (PBSs) with photosystems and possible changes in the thylakoid membrane ultrastructure. Two model organisms Synechocystis 6803 (hereafter Synechocystis) and Synechococcus 7942 (hereafter Synechococcus) are used to investigate state transitions. In a collaboration work, with the Biophysics of Photosynthesis group at the Vrije Universiteit Amsterdam, remodeling of the photosynthetic machinery of two cyanobacteria species, namely, Chlorogloepsis fritschi PCC 6912 (CF) and Chroococcidiopsis thermalis PCC 7203 (CT) as a result of acclimation to the far-red light is investigated. Picosecond time-resolved fluorescence spectroscopy and fluorescence lifetime imaging microscopy are the main techniques used in this work.

\section{Cyanobacteria and oxygenic photosynthesis}

Cyanobacteria, also known as blue green algae, are the only phototrophic prokaryotes capable of performing oxygenic photosynthesis ${ }^{[37]}$. Large scale emergence of cyanobacteria gave sharp rise to the levels of oxygen in the atmosphere and oceans ${ }^{[37-40]}$, changed the character of life from unicellular anaerobic to predominantly multicellular and aerobic ${ }^{[41-43]}$, shaped the geographical and geochemical features of the Earth ${ }^{[37,44,45]}$ and in an endosymbiotic event cyanobacteria presumably became integrated into the chloroplasts of ancestral algae and plants ${ }^{[46-53]}$. To date, cyanobacteria are major players with regard to the carbon cycle in the oceans ${ }^{[54-57]}$ and release a significant amount of oxygen ${ }^{[58]}$. Some species of cyanobacteria are known to fix atmospheric nitrogen ${ }^{[57,59]}$. Cyanobacteria, generally grow much faster than plants, show a high degree of tolerance to extreme environments allowing them to flourish anywhere where at least some water is found ranging from volcanic ashes to polar environments ${ }^{[60-62]}$ and can rapidly adjust their photosynthetic pigment content to the growth conditions. These characteristics make cyanobacteria the organisms of choice for numerous industrial applications ${ }^{[63]}$.

Although many steps in photosynthesis are rather well understood from the light absorption to the reduction of $\mathrm{NADP}^{+}$and synthesis of ATP, the regulatory mechanisms which ensure the safety of photosynthetic apparatus and optimal photosynthetic efficiency still demand considerable investigation. Understanding of regulatory mechanisms in photosynthetic organisms is of great significance to truly appreciate the working dynamics of the 
photosynthetic system as a whole in response to the environmental stimuli. Moreover, a deeper insight into the working mechanisms of the photosynthetic apparatus might enable us to improve the productivity of the natural systems and design better photosynthetic systems.

Cyanobacteria, due to their relative simplicity and ease of genetic manipulation as compared to higher plants, are very suitable model organisms for exploring the molecular basis of the regulatory mechanisms in photosynthetic organisms ${ }^{[64,65]}$.

\section{Photosynthesis in cyanobacteria}

In oxygenic photosynthesis, a set of redox chemical reactions powered by solar radiation assimilate $\mathrm{CO}_{2}$ and hydrogen extracted from water into organic matter, while molecular oxygen is released. A schematic representation of the main components of the photosynthetic machinery involved in light-dependent reactions are shown embedded in the thylakoid membrane of a cyanobacterium in Fig. 1. The thylakoid membrane of a cyanobacterium is a lipid-bilayer of $\sim 5 \mathrm{~nm}$ thick and it is the site for all light reactions of photosynthesis and respiratory processes.

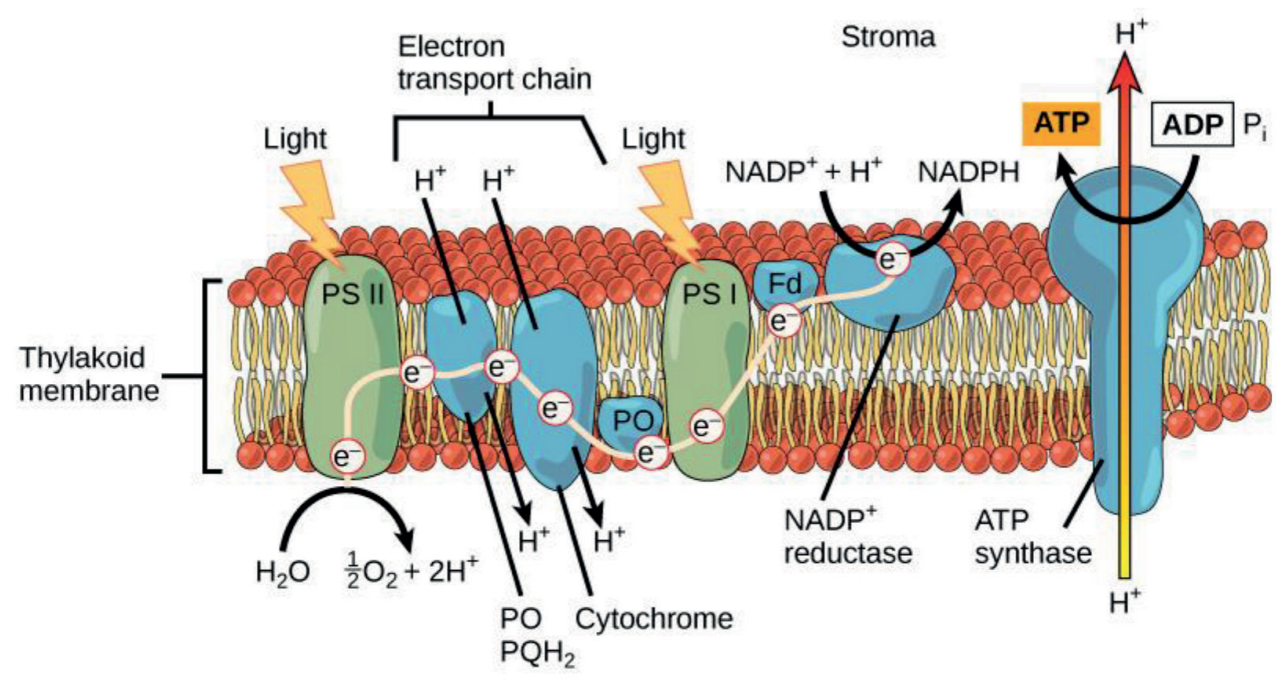

Thylakoid space

Fig. 1. Schematics of the light-induced electron transfer process and the components involved in the cyanobacterial thylakoid membrane. Figure reproduced from the source: https://opentextbc.ca/biology/ chapter/5-2-the-light-dependent-reactions-of-photosynthesis/.

The fundamental step in the conversion of solar energy into chemical energy is the light-induced charge separation (CS) in the reaction centers of photosystem I (PSI) and 
photosystem II (PSII) ${ }^{[6]}$. Direct/indirect excitation of a primary electron donor, presumably P680 in the PSII reaction center converts P680 into the highly reducing agent P680* and consequently an electron is transferred from this primary electron donor to a nearby pheophytin molecule. Charge transfer from the PSII RC Chl is stabilized by the rapid transfer of the electron from pheophytin to the electron transport chain spanning between PSII and PSI. Meanwhile, electrons released from the oxidation of water in the oxygen evolving complex (OEC) of PSIl continuously re-reduce the oxidized $\mathrm{P} 60^{+}$, thereby facilitating a linear flow of electrons from PSII to PSI. At PSI level due the photoexcitation an electron is released, presumably from P700, finally converting $\mathrm{P} 700$ into oxidizing agent $\mathrm{P} 700^{+}$. An electron from PSII, moving from molecule to molecule on its way to PSI keeps ending up at a lower energy level at each transfer step until it reduces the $\mathrm{P} \mathrm{O0}^{+}$in PSI. The electron which is released from P700 due to the photoexcitation in the end reduces $\mathrm{NADP}^{+}$to $\mathrm{NADPH}$. Therefore, for the flow of electrons from water to the final accepter NADPH, both photosystems must work in series. Splitting of two water molecules in the OEC releases molecular oxygen into the atmosphere and the transport of protons and electrons over the membrane by plastoquinol adds up to the proton gradient across the thylakoid membrane. This gradient of protons, or proton motive force, drives the ATP synthase to synthesize ATP from ADP and inorganic-phosphate and during this process protons move from the lumenal side to the stromal side of the membrane. ATP and NADPH produced in the light-dependent redox reactions are later used in light-independent reactions to produce the hydrocarbons. A brief description of the photosystems, cytochrome $b_{6} f$ and ATP synthase is provided in the sections below.

\section{Photosystems}

The photosystems are indispensable for photosynthesis. These complexes form the sites of light harvesting and primary photochemistry, maintain a flow of electrons and gradient of protons across the thylakoid membrane, dynamically adjust the rate of photosynthesis according to cellular needs under stress conditions and have mechanisms to protect and to some extent recover from photodamage. To perform all these tasks, photosystems have necessarily evolved to complex multi-unit systems of protein matrices and associated cofactors. The unique arrangement of protein subunits and cofactors in a photosystem (also regarding the lumenal and stromal side of the membrane) makes the successful functioning of the whole complex as a functionally and physically stable unit in the photosynthesis process possible. Therefore, there is a long-standing interest in photosynthesis research to resolve the number, arrangement, composition and functioning of the protein subunits and their associated cofactors in the photosystems. Structure determinations from Thermosynechococcus elongatus of PSI at $2.5 \AA$ by Jordan

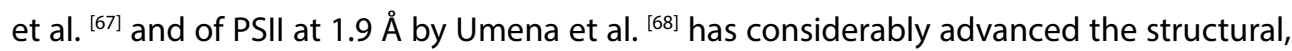
functional and compositional understanding of the complexes and confirmed many interpretations of existing biochemical and biophysical data. 


\section{PSI trimer}

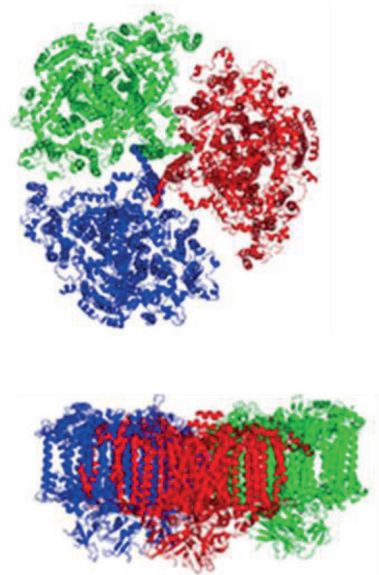

\section{PSI monomer}

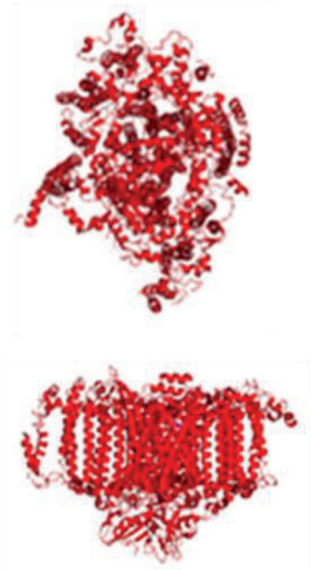

Fig. 2. The crystal structures of PS I trimer (left) and monomer (right) of T. elongatus. Upper panels: top views from the luminal side. Lower panels: lateral views. Different colors represent different monomers of one trimer. The figures are adopted from the crystal structure obtained by X-ray diffraction (Jordan et al. 2001).

The PSI core is reported to be in monomeric, trimeric and tetrameric forms in different cyanobacteria (Fig. 2) ${ }^{[69]}$. PSI of Thermosynechococcus elongatus is comprised of 12 major and minor protein subunits and 127 cofactors, namely, $96 \mathrm{Chl} a, 22 \beta$-carotenes, two phylloquinones, three iron-sulfur clusters $\left(\mathrm{F}_{\mathrm{x}^{\prime}} \mathrm{F}_{\mathrm{A}}\right.$ and, $\left.\mathrm{F}_{\mathrm{B}}\right)$ and four lipids per monomer ${ }^{[58,67] \text {. }}$ The two largest protein subunits of PSI, PsaA and PsaB, coordinating most of the PSI Chls (79 out of 90 antenna Chls $a$ ) and components of the electron transfer chain (ETC), form the heterodimeric core of PSI [58]. At the heart of the ETC is the primary electron donor P700, a chlorophyll dimer, with its absorption maximum at $700 \mathrm{~nm}$. Other components of the ETC chain are a Chl $a$ molecule, a phylloquinone and three iron-sulfide clusters ${ }^{[58]}$. Iron-sulfide cluster $F_{x}$ also has an important role in the assembly and structural integrity of PSI. For $\beta$-carotenes several functions have been determined, ranging from fine-tuning of absorption spectra and photoprotection to structure stabilization ${ }^{[58,70]}$.

PSIl exists in a dimeric form ${ }^{[71-73]}$, where each monomer is comprised of 20 protein subunits, $35 \mathrm{Chl} a$ and $12 \beta$-carotenes (Fig. 3a) ${ }^{[74,75]}$. The most important functional components of the PSII monomers are the RC and the oxygen- evolving/water oxidizing complex (Fig. 3b). At the center of the PSIl complex, D1 and D2 polypeptides comprising the RC proteins are flanked on both sides by one of the core antenna complexes CP43 and CP47 ${ }^{[76]}$. D1 and D2 polypeptides bind and coordinate other RC components, which include six Chls (including P680), two pheophytin molecules ${ }^{[77]}$, cytochrome b559 ${ }^{[78]}$ and a Psbl gene product ${ }^{[79]}$. The light-induced $\mathrm{P} 680^{+}$is a strong oxidizing agent, and via a redox-active tyrosine intermediate it causes the oxidation of water in the oxygen-evolving complex of PSII where the $\mathrm{Mn}_{4} \mathrm{CaO}_{5}$ cluster plays an important role as a catalyst ${ }^{[80,81]}$. PSII is the only 
known biological system that is capable of oxidizing oxygen during the water splitting process.

PSII dimer

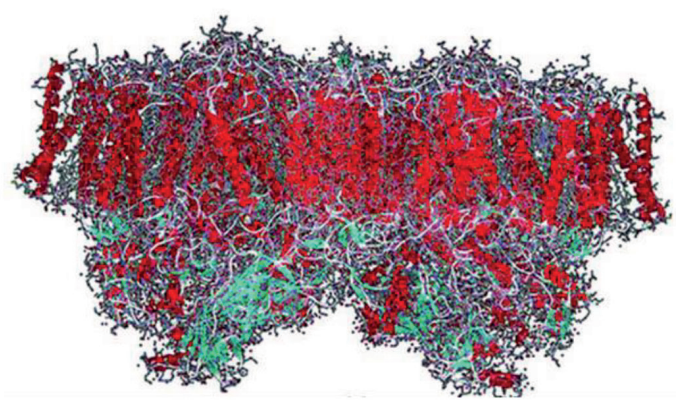

(a)
OEC

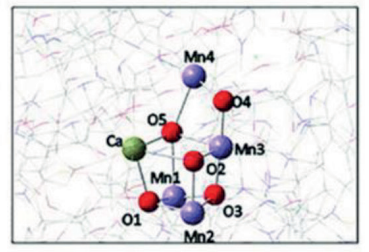

(b)

Fig. 3. General structure of PSII dimer from Thermosynechococcus vulcanus cyanobacteria at 1.9 $\AA$ (PDB: 3ARC) (a) and the OEC (b) present in this protein complex ${ }^{[82]}$.

\section{Cytochrome bf}

The electron transport between the photosystems and the transmembrane transport of protons are at the basis of the photosynthetic light-dependent reactions. The cytochrome $b_{6} f$ complex provides an electronic connection between the photosystems and the transport of the electrons through this complex is coupled with the migration of protons from the stromal to the lumenal side of the membrane ${ }^{[83,84]}$. The structural determination at $3.0 \AA$ A resolution of cytochrome $b_{6} f$ from the thermophilic cyanobacterium Mastigocladus laminsus ${ }^{[85]}$ has revealed the likely pathways for linear electron transport and proton translocation ${ }^{[86]}$. A dimeric structure of the complex from the cyanobacterium Nostoc sp. PCC 7120 is shown in Fig. 4 A.

\section{ATP synthase}

ATP is a universal biological fuel which is synthesized in cells ${ }^{[87,88]}$ and chloroplasts by a large membrane bound enzyme complex called ATP synthase (Fig. 4) ${ }^{[88]}$. The working principle of ATP synthase is of a generator, where the torque generated by the first molecular motor $F_{0}$ driven by the proton motive force drives the second motor $F_{1}$ through a shaft/rotor in the complex to produce ATP from ADP and inorganic phosphate ${ }^{[89,}{ }^{90]}$. In photosynthetic organisms the transmembrane proton gradient generated by the photosystems and maintained by cytochrome $b_{6} f$ drives the ATP-synthase enzyme ${ }^{[88]}$. 
A

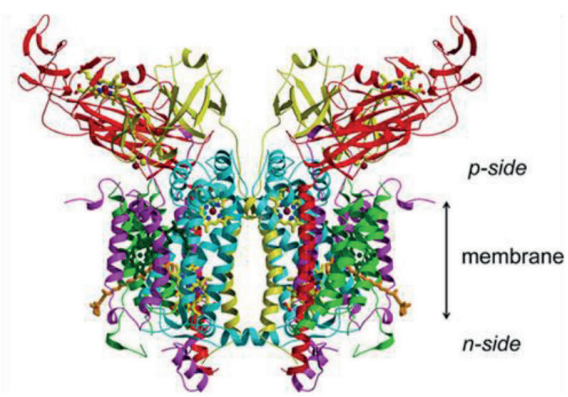

B

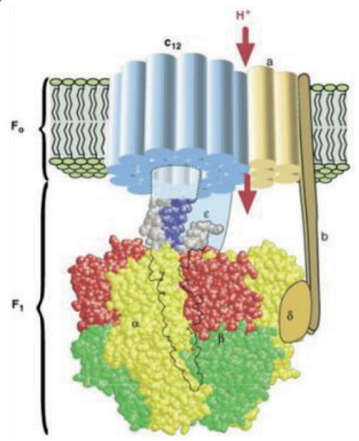

Fig. 4. (A) Crystal structure of cytochrome $b_{6} f$ dimer at $3.0 \AA$ resolution from cyanobacterium Nostoc sp. PCC 7120 adapted from Baniulis et al. (B) a cartoon of ATP synthase adopted from ${ }^{[91]}$.

\section{Light harvesting and energy transfers in cyanobacteria}

Primary photochemistry is an efficient process and consumes fast (within tens to hundreds of picoseconds) the energy of the harvested light. Solar irradiance, despite its abundance, is a dilute source of energy per chlorophyll molecule in view of the fast photochemistry of the photosystems. To keep up with the metabolic demands, cyanobacteria must harvest a many time more light than the Chls in the RCs are capable of. Cyanobacteria, increase their light harvesting capacity by using membrane-embedded and membrane external photosynthetic pigments at the level of both photosystems ${ }^{[66]}$.

\section{Membrane-embedded antenna}

Both PSI and PSII contain a large amount of Chls $a$ which are not part of the photosynthetic electron transfer chain and surround the RC proteins. These pools of energetically interconnected Chls function as light-harvesting antennae and enhance the light-harvesting capacity of the RC Chls ${ }^{[76]}$. The PSII core has two distinct pigment protein complexes, namely, CP43 containing $13 \mathrm{Chls}$ and, CP47 containing $16 \mathrm{Chls}$. The arrangement of pigments is such that the EET from the antenna Chls to the RC Chls is efficient whereas the oxidation of the antenna Chls due to the charge separation in the $\mathrm{RC}$ is not possible ${ }^{[92]}$.

The PSI core harbors $\sim 100$ Chls (including 6 RC Chls) and a small quantity of red-shifted Chls a (absorption maxima above P700) is a conserved feature of this photosystem ${ }^{[93]}$. The presence of low-energy Chls necessitates uphill energy transfer and the organisms with the highest amount of low-energy Chls have the PSI trapping rate of $\sim 50$ ps as compared to $\sim 20$ ps in those organisms which have very low amount of low-energy Chls ${ }^{[94,95]}$. In the last decade some species of cyanobacteria have been shown to be able to remodel their photosynthetic apparatus, incorporating the most red-shifted pigments, $\mathrm{Chl} d$ and $\mathrm{Chl} f$ in their photosystems (PSI and PSII) which are synthesized in a far-red light environment 
[29-35]. Determining the sites of the low-energy Chls $a$ in PSI and Chl $d$ and $\mathrm{Chl} f$ in the photosystems cores of far-red light adapted strains is challenging due to the fast (subpicoseconds) equilibrium of the excitation energy in the large pool of antenna pigments. Therefore, there is still some uncertainty particularly about the incorporation of $\mathrm{Chl} f$ in the RCs.

\section{Phycobilisome (PBS) complexes}

In the photosynthetically active region of the light spectrum, there is a large quantity of photons in the $500-600 \mathrm{~nm}$ range which is only weakly absorbed by the Chls ${ }^{[96]}$. Cyanobacteria increase their light harvesting in this range by using the phycocyanobilin pigments embedded in protein structures, called phycobilisomes (PBSs) ${ }^{[96]}$, externally attached to the stromal side of the thylakoid membrane. The PBS antenna was first

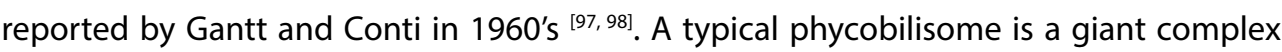
and the structural determination of the whole intact complex with good resolution of its components has been a daunting task. A complete structure of a PBS from the red alga Griffithsia pacifica has recently been resolved ${ }^{[99]}$. A classic model of a PBS has two main substructures, a core composed of 2-5 cylinders and rods, typically up to six, radiating outwards from the core. A schematic of phycobilisome structure of Synechocystis adopted from Dartnell et al ${ }^{[106]}$ is shown in Fig.5. Synechococcus and Synechocystis have bi-and tricylindrical cores, respectively ${ }^{[100-103]}$. At the basis of the PBS self-assembly is the stable complex formation of an $a$ - and a $\beta$-phycobiliprotein (PBP). Three such PBPs form a trimer, forming the building blocks of the rods and core of the PBS antenna. The PBP trimers of PBP join to form hexamers and the stacking of the hexamers determines the length of the PBS rods ${ }^{[101]}$. In the case of Synechococcus and Synechocystis rods contain phycocyanin pigments (PC). Typically, four discs of allophycocyanin (APC) trimers join to make a cylinder in the PBS core. Non-pigmented polypeptides termed as linkers, hold the subunits together, join the rods with the core and provide a link for the interaction of PBS core with the thylakoid membrane and the photosystems ${ }^{[104,105]}$. In the basal cylinders, in one trimer an $\alpha^{A P C}$ subunit is replaced with an ApcD gene product, in another trimer a $\beta^{A P C}$ subunit is replaced with an ApcE gene product and another $\mathrm{a}^{\mathrm{APC}}$ subunit is replaced with an ApcF gene product. These gene products are linker polypeptides and the gene product of ApcE is found essential for the assembly of the PBS core and linking the PBS core with the membrane. ApcD, ApcE and ApcF gene products are the most red-shifted trimers of the APC core emitting at $\sim 680 \mathrm{~nm}$ and are also called terminal emitters. In this work wildtype (WT) strains of Synechococcus and Synechocystis and the strains lacking either the ApcD or ApcF subunits are used. 


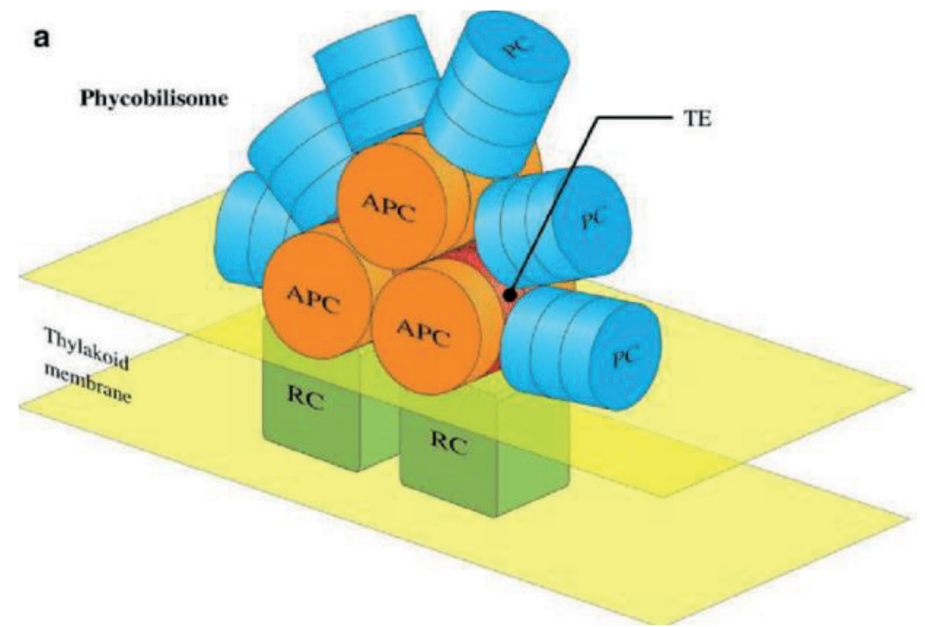

b

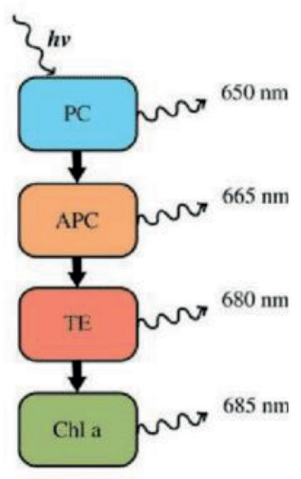

Fig. 5. A schematic of phycobilisome structure of Synechocystis adopted from Dartnell et al [106]. (a) Six rods of phycocyanin (PC) form a hemidiscoidal array and attach to the three cylinders of allophycocyanin core (APC). Two basal cylinders of core bind with the PSII dimer in thylakoid membrane. In (b) excitation of PC and a stepwise excitation-energy transfer from excited-state PC pigment to $\mathrm{Chl} a$ is shown. Fluorescence emission wavelengths from $\mathrm{PC}, \mathrm{APC}$, terminal emitter (TE) and $\mathrm{Chl}$ a are also shown.

The PBS antenna is a marvelous example where the architecture and design confer a layer of tunable photophysical functionalities to the complex. Phycobilin pigments are open chain tetrapyrroles, that tend to fold in a cyclohelical way with generally poor conjugation between adjacent double bonds, and as such free phycobilins generally show strong absorption in the UV and very little absorption in the visible range. However, inside the PBS antenna, the covalently attached phycobilins are held open by the protein structure allowing them to strongly absorb the visible light ${ }^{[107]}$. The optical properties of the bilins are further tuned in the rods and core by the protein environment, linker polypeptides and the assembly of the subunits ${ }^{[96]}$. The ability of PBPs to quickly self-assemble and disassemble give the PBSs the functionality to quickly respond to the environmental changes ${ }^{[108,109]}$ and metabolic stress ${ }^{[110]}$. PBSs can adapt to the environmental conditions and keeping the same architectural principle can increase/decrease the length of the rods and pigment composition ${ }^{[96,111]}$. Due to these tunable photophysical properties, PBSs are important for many regulatory mechanisms.

\section{Excitation energy transfers in cyanobacteria}

An incident photon with the energy in resonance with the bandgap energy of the pigment molecules, can be absorbed and brings the molecule from the singlet ground state $\mathrm{S}_{0}$ to an allowed excited state $S_{n}$. The higher singlet states $\left(S_{2} \ldots S_{n}\right)$ are extremely short lived and through the internal conversion process the pigments relax to the lowest excited state $S_{1}$. From $\mathrm{S}_{1}$ state the molecule transitions to the ground state, either directly or indirectly, via one of the following processes: (i) emitting a photon (fluorescence), (ii) forming a $T_{1}$ triplet 
state due to intersystem crossing (ISC), or (iii) by producing heat (internal conversion). In antenna systems of photosynthetic organisms the presence of several pigments in the vicinity of the excited pigments creates another pathway for depopulation of the excited state, namely excitation-energy transfer (EET) from the excited-state pigment to a neighboring pigment. This non-radiative energy transfer process is called resonance energy transfer from the donor $(D)$ to the acceptor $(A)$ molecule. The distance between the $D$ and $A$ and relative orientations of these pigments is extremely important for the efficiency of the energy transfer. The dipole-dipole interaction $\left(V_{D A}\right)$ between donor $D$ and acceptor $A$ is a coulombic interaction and can be written as:

$$
V_{D A}=\left(\frac{1}{4 \pi \varepsilon \varepsilon_{0}}\right) \cdot \frac{\boldsymbol{\mu}_{D} \cdot \boldsymbol{\mu}_{A}-3\left(\boldsymbol{\mu}_{D} \cdot \boldsymbol{R}\right)\left(\boldsymbol{\mu}_{A} \cdot \boldsymbol{R}\right)}{R_{D A}^{3}}
$$

Here $\boldsymbol{\mu}_{\boldsymbol{D}}$ and $\boldsymbol{\mu}_{\boldsymbol{A}}$ are the transition dipole moments of the donor and acceptor, respectively. $\mathrm{R}_{\mathrm{DA}}$ is the center-to-center distance between $D$ and $A$. $\varepsilon$ is the dielectric constant, $\varepsilon_{O}$ is the vacuum permittivity.

\section{Energy regulatory mechanisms}

The presence of a functional photosynthesis machinery is necessary but not sufficient for continuous and optimal/efficient photosynthesis, required for the sustenance and growth of the organism in various environments and conditions. Light harvesting and excitation energy regulatory mechanisms in photosynthetic organism ensure the much-needed governance for the safety of the photosynthetic apparatus and optimal/ efficient photosynthesis. The need for excitation-energy regulation arises when the light conditions cause an imbalance of excitation between the two photosystems working in series, or when the light intensity exceeds the energy consumption of the organism in nutrient-deficient conditions or, the light intensity is too high such that it leads to the formation of reactive oxygen species. Most of such light conditions can and do occur in a matter of seconds to minutes. Photosynthetic organisms face the unique challenge to operate a light-harvesting apparatus with sufficient absorption cross-section to drive the necessary amount of photosynthesis in low-light conditions, whereas they need to protect themselves when the absorbed light in the next moments becomes too high. At high light intensities, various fast non-photochemical quenching (NPQ) processes convert the excited-state energy into heat to protect the organisms from high light induced damages. One particularly important NPQ process in most cyanobacteria is the reversible blue-green light induced orange carotenoid protein (OCP) quenching. This NPQ process occurs in Synechocystis but not in Synechococcus. It is of potential interest that (see chapter 6) Synechococcus shows a much larger amount of state transitions as compared to Synechocystis. State transitions, the regulatory mechanism that is focused on in this thesis, ensures the balance of photochemistry between the two photosystems. 


\section{Fluorescence and experimental techniques}

Direct/indirect excitation of the chlorophyll molecule stores the solar energy in the form of excitations for a few nanoseconds and facilitate the start of the photochemistry needed for photosynthesis. The fate of the excited primary electron donors of the RCs (P680/P700) is determined by several competing de-excitation processes, namely, photochemistry in the RCs, photon emission(fluorescence), non-photochemical quenching and nonradiative decay to the ground state (internal conversion) or a triplet state (intersystem crossing). Of all these processes, fluorescence is directly observable and reports on the presence and efficiency of the rest of the competing de-excitation pathways. Therefore, the dynamics of the chlorophyll fluorescence is extensively studied, to quantify the light harvesting and photosynthetic efficiency, to identify the initial steps of the photochemistry and to disentangle and determine the nature of the various regulatory and feedback mechanisms. For this thesis the following fluorescence techniques are used: pulse amplitude modulated fluorometry (PAM), time-resolved fluorescence spectroscopy and fluorescence-lifetime-imaging-microscopy (FLIM). A brief discussion about these methods in the context of this thesis is provided below.

\section{Pulse-amplitude-modulated fluorometry (PAM)}

The $\mathrm{Chl} a$ fluorescence signal is rich in information about the photosynthetic performance and physiological state of the organism ${ }^{[12,113]}$. The pulse-amplitude-modulated fluorometry method with the integration of fast electronics and suitable optics in one setup, has made it possible to induce fluorescence under different dark-light conditions and interpret the relative fluorescence changes with great accuracy. The fundamental principle of this method is the use of amplitude-modulated measuring light of a certain frequency which probes the state of the sample and results in the amplitude-modulated fluorescence which is separable from the fluorescence resulting due to the ambient light. In this method three different light sources, namely, the measuring/probe light, the actinic light and saturating light/saturation pulse are used ${ }^{[114,115]}$. The intensity of the measuring light is very low, in order to probe the fluorescence yield of the Chls in dark. The actinic light is used to drive photosynthesis. The saturation pulse of high intensity is used to estimate the maximal and minimal fluorescence yield but also NPQ ${ }^{[16]}$. Analysis of the PAM fluorescence signal allows to obtain information about the kinetics of the electron transport in PSII (photosynthetic activity), the buildup of the transmembrane $\Delta \mathrm{pH}$ due to the photosynthetic activity ${ }^{[117]}$, kinetics of regulatory mechanisms such as state transitions, NPQ and relaxation from the quenched state under different dark-light treatments.

In this work, we have used the PAM for the initial in vivo characterization of the physiological condition of cyanobacteria with regard to their state transitions. The kinetics and the extent of the transition to state $1 /$ II were estimated from the fluorescence traces using 
saturation pulses and the samples in the right physiological condition were later used for the detailed characterization with time-resolved fluorescence spectroscopy.

\section{Time-resolved fluorescence spectroscopy}

Time-resolved fluorescence spectroscopy is a non-invasive method that we used to study the ultrafast processes of light harvesting and regulation in photosynthetic organisms. It provides a window to the functional state of the system by means of its ability to resolve the fluorescence changes with (in our case) picosecond time resolution across the complete emission spectrum. Furthermore, the steady-state fluorescence spectra which describes the structural state of the system, can be reconstructed from the analysis of the time-resolved fluorescence spectra.

Changes in the quality or the intensity of the ambient light often result in changes in the fluorescence emission of the photosynthetic organism. These fluorescence changes can be the result of $i$ ) the modulation of the antenna size/absorption cross-section of the photosystems and/or ii) the modulation of the excited-state dynamics of the photosystems and/or light-harvesting complexes. Fluorescence from a sample is the product of the fluorescence amplitude and lifetime of the excited state. The fluorescence amplitude corresponds to the absorption cross-section/antenna size of the photosynthetic unit. Therefore, with steady-state fluorescence spectroscopy it can be challenging and, in some cases, impossible to distinguish between the scenarios for the observed fluorescence changes. Time-resolved fluorescence spectroscopy, due to its simultaneous access to the information about both the fluorescence amplitude and the excited-state lifetime, can in principle resolve the nature of the mechanism underlying the fluorescence changes observed in changing light conditions. Excited-state dynamics can be probed with other techniques as well, such as, transient absorption spectroscopy. However, timeresolved fluorescence requires far lower light intensities, which makes it suitable to study cyanobacteria in vivo with both open and closed RCs. For these reasons, time-resolved fluorescence spectroscopy was our main technique of choice to investigate the state transitions in cyanobacteria.

To perform the time-resolved fluorescence measurements, a train of short pulses of light (femto/picosecond duration) are used to excite the sample. The interval between the pulses is long enough for most pigments to relax back to the ground state, before the sample is hit again by the next excitation pulse. The resulting fluorescence is gathered and dispersed spatially both with respect to wavelength and time in a well-defined way. We used a synchroscan streak-camera for our measurements. The working principles of excitation pulse generation and synchronized detection of the fluorescence using the streak-camera method are described elsewhere in detail ${ }^{[118]}$. 
Figure 6 shows a streak-camera image of the signature time-resolved fluorescence profile of Synechococcus upon selective excitation of PBSs using $577 \mathrm{~nm}$ pulses. The fluorescence as a function of wavelength and time is shown along the horizontal and vertical axis, respectively. The fluorescence intensity is represented with the color scale provided next to the streak image.

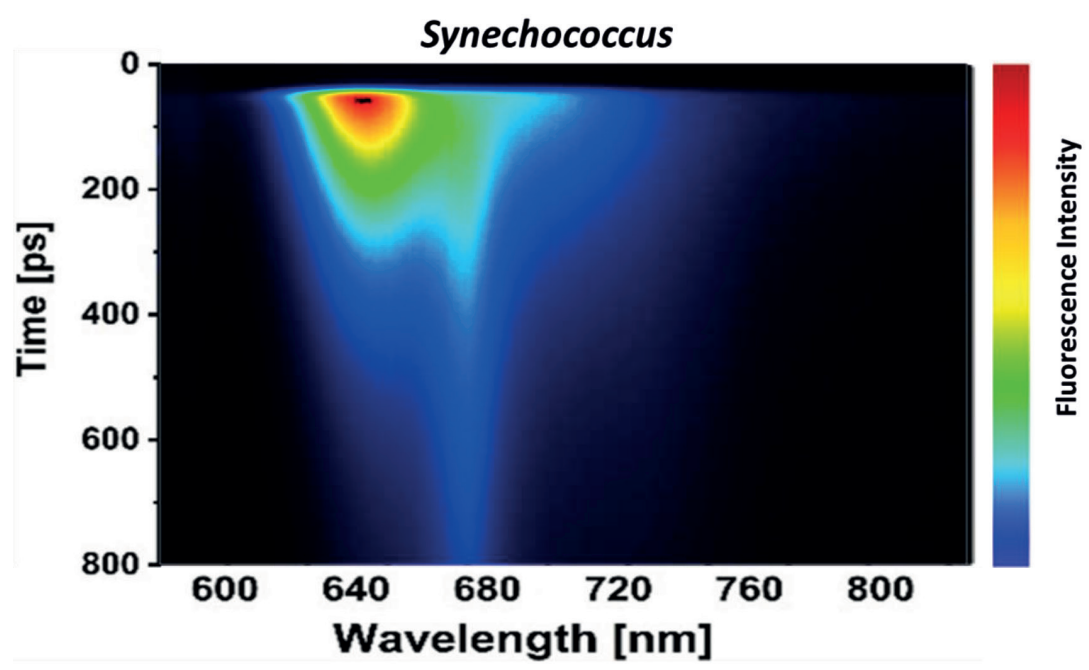

Fig.6. Streak-camera image of time-resolved fluorescence spectra of Synechococcus upon PBS excitation.

The streak-camera detected fluorescence response of the sample (Fig. 6) is the convolution of the time-and wavelength-dependent fluorescence with the time-and wavelength dependent instrument response function (IRF).

The fluorescence trace at each wavelength can be approximated by sum of exponentials:

$$
F(t)=\sum_{1,2 \ldots}^{n} A_{i} \cdot \exp \left(-\frac{t}{\tau_{i}}\right)
$$

Here $\boldsymbol{\tau}_{\mathrm{i}}$ are the fluorescence decay times, $\boldsymbol{A}_{\boldsymbol{i}}$ the corresponding amplitudes and $\boldsymbol{n}$ represents the total number of decay components. The global analysis method has been used throughout this thesis to model the time-resolved fluorescence data. In this method the sample is considered to contain a set of identical fluorophores and the decay curves are fitted simultaneously across the whole emission spectrum by forcing either $\boldsymbol{\tau}_{\mathrm{i}}$ or $\boldsymbol{A}_{\boldsymbol{i}}$ to be identical. Assuming that the fluorescence lifetimes $\boldsymbol{\tau}_{\mathrm{i}}$ are identical across the whole emission spectrum (or forced to be the same), the recorded time-resolved fluorescence can be fitted with the following expression.

$$
F(\lambda, t)=\sum_{1,2 \ldots}^{n} A_{i}(\lambda) \cdot \exp \left(-\frac{t}{\tau_{i}}\right) \otimes \operatorname{IRF}(\lambda, t)
$$

Throughout the thesis $\boldsymbol{A}_{\boldsymbol{i}}(\lambda)$ are called decay associated spectra (DAS). 


\section{Fluorescence lifetime imaging microscopy}

In cyanobacteria the mechanism of state transitions balances the photochemistry of photosystem I (PSI) and photosystem II (PSII) when one photosystem is overexcited relative to the other. If PSI is preferentially excited, cells go to state I whereas state II is induced either after preferential excitation of PSII or after dark adaptation. The streak-camera method allows to probe the excited-state dynamics for an ensemble of cyanobacteria in state I and II with excellent temporal and spectral resolution. However, it does not allow to map the fluorescence kinetics at the cellular level. Fluorescence lifetime imaging microscopy (FLIM) is used in this work to complement the investigation of state transitions at the ensemble level with that at the cellular level.

With this FLIM method, the image of the specimen is constructed from the fluorescence lifetime contrast instead of the contrast in intensity. The lifetime of the excited state is a parameter that is independent of factors such as probelight and fluorophore concentrations and photobleaching (under right scanning conditions). On the other hand, the excitedstate dynamics of a fluorophore is sensitive to the local environment, for instance the local $\mathrm{pH}$ and the energetic coupling to neighboring complexes. Furthermore, fluorescent species with overlapping emission spectra like PSI and PSII can be distinguished based on their distinct lifetimes. Therefore, FLIM is an excellent tool to image the functional, structural, compositional and distributional inhomogeneities at the (sub)-cellular level, in contrast to the ensemble spectroscopy studies but also intensity-based fluorescence microscopy. It is this strength of the FLIM which is exploited in this work to image the distribution of photosystems and state transitions in individual cells of Synechococcus.

FLIM data can in general be acquired in two different ways, either the time-domain or the frequency-domain method. Both methods differ in their hardware but are mathematically equivalent and the data acquired in one domain can be converted to the other. In this work, the time-correlated single photon counting method (TCSPC) is used to perform fluorescence lifetime imaging. The fundamentals of this method are explained below. Picosecond laser pulses with a certain repetition rate are used to excite the sample and the arrival time of the emitted photon at the detector is registered with respect to the trigger time of the excitation pulse. The difference between the timings of the trigger signal and detector signal is calculated. The measurement is repeated until the histogram containing the time information of the arrival of photons has sufficient number of counts for the estimation of the fluorescence decay. The schematics of the TCSPC principle for the estimation of the fluorescence decay is shown in the Fig.7 ${ }^{[119]}$. 

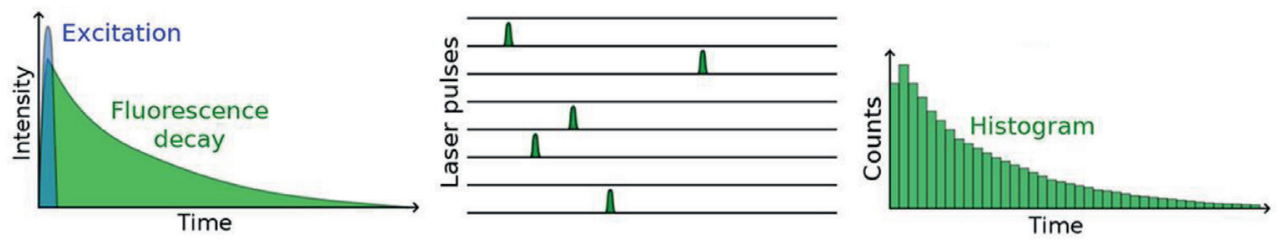

Fig.7. The schematics of the TCSPC principle for the estimation of fluorescence decay from a sample upon excitation by laser pulses. The figure is reproduced from the work of Hirvonen and Suhling ${ }^{[119]}$.

In FLIM the fluorescence decay is measured for each pixel of the image as shown in Fig. $8^{[119]}$. The fluorescence decay acquired in FLIM data is often fitted with multi exponential decays for each pixel. The important consideration in this method is to avoid the detection of more than one photon per excitation pulse period. If the intensity is high, such that more than one photon often arrives at the detector during one signal period, it results in an erroneous histogram of the arrival time of the first photon, leading to an inaccurate estimation of the fluorescence decay.
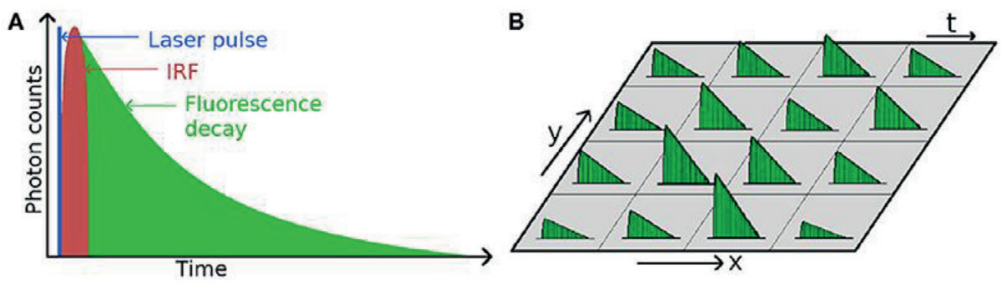

Fig.8. Estimation of fluorescence lifetime in each pixel with TCSPC principle. The figure is reproduced from the work in ${ }^{[119]}$.

\section{Outline of this thesis}

Chapter 2: In this chapter state transitions in wild-type Synechococcus and Synechocystis and mutants lacking $A p c D$ and $A p c F$ proteins are characterized based upon their picosecond fluorescence kinetics at room temperature. The purpose of this work is to remove the existing controversies about the difference in the redistribution of PBS- and Chl-a-absorbed excitation energy during state transitions.

Chapter 3: In this chapter the involvement of PBS complexes in the process of state transitions is investigated by using high osmotic strength buffers which were shown in literature to be able to control PBS diffusion. State transitions kinetics are monitored as a function of buffer strength (which correlates with PBS mobility) both upon PBS and $\mathrm{Chl} a$ excitation to rule out/confirm the presence of more than one process involved in state transitions. Furthermore, insight is provided in the interaction of PBSs with the photosystems. An active role of the thylakoid membrane during state transitions is also discussed. A model for state transitions in cyanobacteria is provided to explain the results shown in chapters 2 and 3. 
Chapter 4: In this chapter fluorescence lifetime imaging microscopy is used to map the global (re-) distribution of photosystems before and after state transitions process at the cellular level. FLIM is used to test whether i) photosystems show different distributions in the dark- and light-adapted cells and ii) membrane changes during state transitions as proposed in chapter 3 can be observed.

Chapter 5: In this chapter time-resolved fluorescence spectroscopy is used to i) quantify the efficiency of light conversion after selective excitation of far-red light PBSs (FRL-PBSs) and far-red light photosystems (FRL-PS) and ii) probe the connectivity of FRL-PBSs with the FRL-PS photosystems in two FaRLip acclimated strains.

Chapter 6: The work presented in chapters 2-5 is broadly discussed.

Chapter 7: A summary of the thesis is provided. 


\section{References}

1. Croce, R. and H. van Amerongen, Light harvesting in oxygenic photosynthesis: Structural biology meets spectroscopy. Science, 2020. 369(6506): p. eaay2058.

2. Sánchez-Baracaldo, P. and T. Cardona, On the origin of oxygenic photosynthesis and Cyanobacteria. New Phytologist, 2020. 225(4): p. 1440-1446.

3. Schirrmeister, B.E., P. Sanchez-Baracaldo, and D. Wacey, Cyanobacterial evolution during the Precambrian. International Journal of Astrobiology, 2016. 15: p. 187.

4. Dismukes, G.C., et al., The origin of atmospheric oxygen on Earth: the innovation of oxygenic photosynthesis. Proceedings of the National Academy of Sciences of the United States of America, 2001. 98(5): p. 2170-2175.

5. Hartman, H., Photosynthesis and the origin of life. Orig Life Evol Biosph, 1998. 28(4-6): p. 515-21.

6. Li, Z., et al., Sensing and Responding to Excess Light. Annual Review of Plant Biology, 2009. 60(1): p. 239-260.

7. Graham, P.J., et al., A penalty on photosynthetic growth in fluctuating light. Scientific Reports, 2017. 7(1): p. 12513.

8. Schumann, T., et al., Plant Growth under Natural Light Conditions Provides Highly Flexible ShortTerm Acclimation Properties toward High Light Stress. Frontiers in Plant Science, 2017. 8(681).

9. Müller, P., X.-P. Li, and K.K. Niyogi, Non-Photochemical Quenching. A Response to Excess Light Energy1. Plant Physiology, 2001. 125(4): p. 1558-1566.

10. Jahns, P. and A.R. Holzwarth, The role of the xanthophyll cycle and of lutein in photoprotection of photosystem II. Biochimica et Biophysica Acta (BBA) - Bioenergetics, 2012. 1817(1): p. 182-193.

11. Ruban, A.V., M.P. Johnson, and C.D.P. Duffy, The photoprotective molecular switch in the photosystem Il antenna. Biochimica et Biophysica Acta (BBA) - Bioenergetics, 2012. 1817(1): p. 167-181.

12. Derks, A., K. Schaven, and D. Bruce, Diverse mechanisms for photoprotection in photosynthesis. Dynamic regulation of photosystem II excitation in response to rapid environmental change. Biochimica et Biophysica Acta (BBA) - Bioenergetics, 2015. 1847(4): p. 468-485.

13. Goss, R. and B. Lepetit, Biodiversity of NPQ. Journal of Plant Physiology, 2015. 172: p. 13-32.

14. Horton, P., M. Wentworth, and A. Ruban, Control of the light harvesting function of chloroplast membranes: The LHCII-aggregation model for non-photochemical quenching. FEBS Letters, 2005. 579(20): p. 4201-4206.

15. Allen, J.F., Protein phosphorylation in regulation of photosynthesis. Biochimica et Biophysica Acta (BBA) - Bioenergetics, 1992. 1098(3): p. 275-335.

16. Bellafiore, S., et al., State transitions and light adaptation require chloroplast thylakoid protein kinase STN7. Nature, 2005. 433(7028): p. 892-895.

17. Minagawa, J., State transitions-The molecular remodeling of photosynthetic supercomplexes that controls energy flow in the chloroplast. Biochimica et Biophysica Acta (BBA) - Bioenergetics, 2011. 1807(8): p. 897-905. 
18. Ünlü, C., et al., State transitions in \&lt;em\&gt;Chlamydomonas reinhardtii\&lt;/em\&gt; strongly modulate the functional size of photosystem II but not of photosystem I. Proceedings of the National Academy of Sciences, 2014. 111(9): p. 3460.

19. Wendler, J. and A.R. Holzwarth, State Transitions in the Green Alga Scenedesmus Obliquus Probed by Time-Resolved Chlorophyll Fluorescence Spectroscopy and Global Data Analysis. Biophysical Journal, 1987. 52(5): p. 717-728.

20. Drop, B., et al., Consequences of state transitions on the structural and functional organization of Photosystem I in the green alga Chlamydomonas reinhardtii. The Plant Journal, 2014. 78(2): p. 181-191.

21. Scheuring, S. and J.N. Sturgis, Chromatic Adaptation of Photosynthetic Membranes. Science, 2005. 309(5733): p. 484.

22. Kehoe, D.M., Chromatic adaptation and the evolution of light color sensing in cyanobacteria. Proceedings of the National Academy of Sciences, 2010. 107(20): p. 9029.

23. Dring, M.J., Chromatic adaptation of photosynthesis in benthic marine algae: An examination of its ecological significance using a theoretical model. Limnology and Oceanography, 1981. 26(2): p. 271-284.

24. Lopez-Figueroa, F., R. Perez, and F.X. Niell, Effects of red and far-red light pulses on the chlorophyll and biliprotein accumulation in the red alga Corallina elongata. Journal of Photochemistry and Photobiology B: Biology, 1989. 4(2): p. 185-193.

25. Ragni, M. and M. Ribera D'Alcalà, Light as an information carrier underwater. Journal of Plankton Research, 2004. 26(4): p. 433-443.

26. Wolf, B.M. and R.E. Blankenship, Far-red light acclimation in diverse oxygenic photosynthetic organisms. Photosynth Res, 2019. 142(3): p. 349-359.

27. Zhen, S. and B. Bugbee, Substituting Far-Red for Traditionally Defined Photosynthetic Photons Results in Equal Canopy Quantum Yield for CO(2) Fixation and Increased Photon Capture During Long-Term Studies: Implications for Re-Defining PAR. Frontiers in plant science, 2020. 11: p. 581156-581156.

28. Sirohiwal, A., et al., Accurate Computation of the Absorption Spectrum of Chlorophyll a with Pair Natural Orbital Coupled Cluster Methods. The Journal of Physical Chemistry B, 2020. 124(40): p. 8761-8771.

29. Gan, F., et al., Extensive remodeling of a cyanobacterial photosynthetic apparatus in far-red light. Science, 2014. 345(6202): p. 1312.

30. Gan, F. and D.A. Bryant, Adaptive and acclimative responses of cyanobacteria to far-red light. Environmental Microbiology, 2015. 17(10): p. 3450-3465.

31. Gan, F., G. Shen, and D.A. Bryant, Occurrence of Far-Red Light Photoacclimation (FaRLiP) in Diverse Cyanobacteria. Life, 2015. 5(1).

32. Kühl, M., et al., Substantial near-infrared radiation-driven photosynthesis of chlorophyll f-containing cyanobacteria in a natural habitat. eLife, 2020. 9: p. e50871. 
33. Zhang, Z.-C., et al., Widespread occurrence and unexpected diversity of red-shifted chlorophyll producing cyanobacteria in humid subtropical forest ecosystems. Environmental Microbiology, 2019. 21(4): p. 1497-1510.

34. Miyashita, $\mathrm{H}_{\text {., }}$ et al., Discovery of chlorophyll $d$ in Acaryochloris marina and chlorophyll $f$ in a unicellular cyanobacterium, strain KC1, isolated from Lake Biwa. Journal of Physical Chemistry \& Biophysics, 2014. 4(4): p. 1.

35. Behrendt, L., et al., Endolithic chlorophyll d-containing phototrophs. The ISME journal, 2011. 5(6): p. 1072-1076.

36. Mascoli, V., L. Bersanini, and R. Croce, Far-red absorption and light-use efficiency trade-offs in chlorophyll f photosynthesis. Nature Plants, 2020. 6(8): p. 1044-1053.

37. Demoulin, C.F., et al., Cyanobacteria evolution: Insight from the fossil record. Free Radical Biology and Medicine, 2019. 140: p. 206-223.

38. Peschek, G.A., Photosynthesis and Respiration of Cyanobacteria, in The Phototrophic Prokaryotes, G.A. Peschek, W. Löffelhardt, and G. Schmetterer, Editors. 1999, Springer US: Boston, MA. p. 201-209.

39. Bekker, A., et al., Dating the rise of atmospheric oxygen. Nature, 2004. 427(6970): p. 117-120.

40. Hannah, J.L., et al., Primitive Os and 2316Ma age formarine shale: implications for Paleoproterozoic glacial events and the rise of atmospheric oxygen. Earth and Planetary Science Letters, 2004. 225(1-2): p. 43-52.

41. Soo, R.M., et al., On the origins ofoxygenic photosynthesis and aerobic respiration in Cyanobacteria. Science, 2017. 355(6332): p. 1436.

42. Dismukes, G.C., et al., The origin of atmospheric oxygen on Earth: the innovation of oxygenic photosynthesis. Proceedings of the National Academy of Sciences, 2001. 98(5): p. 2170-2175.

43. Falkowski, P.G., Tracing oxygen's imprint on Earth's metabolic evolution. Science, 2006. 311 (5768): p. 1724-1725.

44. Sánchez-Baracaldo, P., Origin of marine planktonic cyanobacteria. Scientific Reports, 2015. 5(1): p. 17418.

45. Lucht, W., Earth systems: Shaped by life. Nature, 2011. 470(7335): p. 460-461.

46. Bhattacharya, D. and L. Medlin, The phylogeny of plastids: a review based on comparisons of small-subunit ribosomal RNA coding regions. Journal of Phycology, 1995. 31(4): p. 489-498.

47. DELwicHE, C.F., M. KUHsEL, and J.D. Palmer, Phylogenetic analysis of tufA sequences indicates a cyanobacterial origin of all plastids. Molecular phylogenetics and evolution, 1995. 4(2): p. 110128.

48. Douglas, S.E., Plastid evolution: origins, diversity, trends. Current opinion in genetics \& development, 1998. 8(6): p. 655-661.

49. Hackett, J.D., et al., Plastid endosymbiosis: sources and timing of the major events. Evolution of primary producers in the sea, 2007: p. 109-132.

50. Rodríguez-Ezpeleta, N., et al., Monophyly of primary photosynthetic eukaryotes: green plants, red algae, and glaucophytes. Current biology, 2005. 15(14): p. 1325-1330. 
51. Falcón, L.I., S. Magallón, and A. Castillo, Dating the cyanobacterial ancestor of the chloroplast. The ISME Journal, 2010. 4(6): p. 777-783.

52. Raven, J.A. and J.F. Allen, Genomics and chloroplast evolution: what did cyanobacteria do for plants? Genome biology, 2003. 4(3): p. 1-5.

53. Martin, W., et al., Evolutionary analysis of Arabidopsis, cyanobacterial, and chloroplast genomes reveals plastid phylogeny and thousands of cyanobacterial genes in the nucleus. Proceedings of the National Academy of Sciences, 2002. 99(19): p. 12246-12251.

54. Berla, B.M., et al., Synthetic biology of cyanobacteria: unique challenges and opportunities. Frontiers in microbiology, 2013. 4: p. 246.

55. Case, A.E. and S. Atsumi, Cyanobacterial chemical production. Journal of biotechnology, 2016. 231: p. 106-114.

56. Nozzi, N.E., J.W. Oliver, and S. Atsumi, Cyanobacteria as a platform for biofuel production. Frontiers in bioengineering and biotechnology, 2013. 1: p. 7.

57. Fuchsman, C.A., et al., Cyanobacteria and cyanophage contributions to carbon and nitrogen cycling in an oligotrophic oxygen-deficient zone. The ISME Journal, 2019. 13(11): p. 2714-2726.

58. Fromme, P., P. Jordan, and N. Krauß, Structure of photosystem I. Biochimica et Biophysica Acta (BBA) - Bioenergetics, 2001. 1507(1): p. 5-31.

59. Paerl, H., The cyanobacterial nitrogen fixation paradox in natural waters. F1000Research, 2017. 6: p. 244-244.

60. Schirrmeister, B.E., et al., Evolution of multicellularity coincided with increased diversification of cyanobacteria and the Great Oxidation Event. Proceedings of the National Academy of Sciences, 2013. 110(5): p. 1791.

61. Sanchez-Baracaldo, P., P. Hayes, and C.E. BLANK, Morphological and habitat evolution in the Cyanobacteria using a compartmentalization approach. Geobiology, 2005. 3(3): p. 145-165.

62. Gerasimenko, L.M., et al., Interaction of cyanobacteria with volcanic ashes. Microbiology, 2013. 82(1): p. 111-118.

63. Garlapati, D., et al., Role of cyanobacteria in agricultural and industrial sectors: an outlook on economically important byproducts. Applied Microbiology and Biotechnology, 2019. 103.

64. Ikeuchi, M. and S. Tabata, Synechocystis sp. PCC 6803 - a useful tool in the study of the genetics of cyanobacteria. Photosynthesis Research, 2001. 70(1): p. 73-83.

65. Jensen, P.E. and D. Leister, Cyanobacteria as an Experimental Platform for Modifying Bacterial and Plant Photosynthesis. Frontiers in Bioengineering and Biotechnology, 2014. 2(7).

66. Blankenship, R.E., Molecular mechanisms of photosynthesis. 2021: John Wiley \& Sons.

67. Jordan, P., et al., Three-dimensional structure of cyanobacterial photosystem I at 2.5 Å resolution. Nature, 2001. 411(6840): p. 909-917.

68. Umena, Y., et al., Crystal structure of oxygen-evolving photosystem II at a resolution of $1.9 \AA$. Nature, 2011. 473(7345): p. 55-60.

69. Kato, K., et al., Structure of a cyanobacterial photosystem I tetramer revealed by cryo-electron microscopy. Nature Communications, 2019. 10(1): p. 4929. 
70. Tóth, T.N., et al., Carotenoids are essential for the assembly of cyanobacterial photosynthetic complexes. Biochimica et Biophysica Acta (BBA) - Bioenergetics, 2015. 1847(10): p. 1153-1165.

71. Nelson, N. and A. Ben-Shem, The complex architecture of oxygenic photosynthesis. Nat Rev Mol Cell Biol, 2004. 5(12): p. 971-82.

72. Nickelsen, J. and B. Rengstl, Photosystem II Assembly: From Cyanobacteria to Plants. Annual Review of Plant Biology, 2013. 64(1): p. 609-635.

73. Zlenko, D.V., et al., Coupled rows of PBS cores and PSII dimers in cyanobacteria: symmetry and structure. Photosynth Res, 2017. 133(1-3): p. 245-260.

74. Heinz, S., et al., Analysis of photosystem Il biogenesis in cyanobacteria. Biochimica et Biophysica Acta (BBA) - Bioenergetics, 2016. 1857(3): p. 274-287.

75. Müh, F., T. Renger, and A. Zouni, Crystal structure of cyanobacterial photosystem II at $3.0 \AA$ resolution: $A$ closer look at the antenna system and the small membrane-intrinsic subunits. Plant Physiology and Biochemistry, 2008. 46(3): p. 238-264.

76. Gao, J., et al., Structure and Function of the Photosystem Supercomplexes. Frontiers in Plant Science, 2018. 9(357).

77. Nelson, N. and A. Ben-Shem, The complex architecture of oxygenic photosynthesis. Nature Reviews Molecular Cell Biology, 2004. 5(12): p. 971-982.

78. Guerrero, F., et al., The role of the high potential form of the cytochrome b559: Study of Thermosynechococcus elongatus mutants. Biochimica et Biophysica Acta (BBA) - Bioenergetics, 2014. 1837(6): p. 908-919.

79. Dobáková, M., M. Tichy, and J. Komenda, Role of the Psbl protein in photosystem II assembly and repair in the cyanobacterium Synechocystis sp. PCC 6803. Plant physiology, 2007. 145(4): p. 1681-1691.

80. Glöckner, C., et al., Structural changes of the oxygen-evolving complex in photosystem II during the catalytic cycle. The Journal of biological chemistry, 2013. 288(31): p. 22607-22620.

81. Najafpour, M.M. and Govindjee, Oxygen evolving complex in Photosystem II:Better than excellent. Dalton Transactions, 2011. 40(36): p. 9076-9084.

82. Castilho-Almeida, E.W., et al., Structural Analysis of High-Spin States of SO-S4 at OEC Complex: A Theoretical Approach of Small Models. Journal of the Brazilian Chemical Society, 2017. 28: $p$. 242-249.

83. Baniulis, D., et al., Structure-Function of the Cytochrome b6f Complext. Photochemistry and Photobiology, 2008. 84(6): p. 1349-1358.

84. Baniulis, D., et al., Structure-Function, Stability, and Chemical Modification of the Cyanobacterial Cytochrome b6f Complex from Nostoc sp. PCC 7120. The Journal of biological chemistry, 2009. 284(15): p. 9861-9869.

85. Kurisu, G., et al., Structure of the cytochrome b6f complex of oxygenic photosynthesis: tuning the cavity. Science, 2003. 302(5647): p. 1009-14.

86. Hasan, S.S., et al., Quinone-dependent proton transfer pathways in the photosynthetic cytochrome \&lt;em\&gt;b\&lt;/em\&gt;\&lt;sub\&gt;6\&lt;/sub\&gt;\&lt;em\&gt;f\&lt;/em\&gt; complex. Proceedings of the National Academy of Sciences, 2013. 110(11): p. 4297. 
87. Khakh, B.S. and G. Burnstock, The double life of ATP. Scientific American, 2009. 301(6): p. 84-92.

88. van Walraven, H.S. and R.H.A. Bakels, Function, structure and regulation of cyanobacterial and chloroplast ATP synthase. Physiologia Plantarum, 1996. 96(3): p. 526-532.

89. Okuno, D., R. lino, and H. Noji, Rotation and structure of FoF1-ATP synthase. The Journal of Biochemistry, 2011. 149(6): p. 655-664.

90. Stewart, A.G., et al., Rotary ATPases: models, machine elements and technical specifications. Bioarchitecture, 2013. 3(1): p. 2-12.

91. Oster, G. and H. Wang, ATP synthase: two motors, two fuels. Structure, 1999. 7(4): p. R67-R72.

92. van Amerongen, H. and R. Croce, Light harvesting in photosystem II. Photosynthesis Research, 2013. 116(2): p. 251-263.

93. Toporik, H., et al., The structure of a red-shifted photosystem I reveals a red site in the core antenna. Nature Communications, 2020. 11(1): p. 5279.

94. Gobets, B., et al., Time-Resolved Fluorescence Emission Measurements of Photosystem I Particles of Various Cyanobacteria: A Unified Compartmental Model. Biophysical Journal, 2001. 81(1): p. 407-424.

95. Ihalainen, J.A., et al., Kinetics of excitation trapping in intact Photosystem I of Chlamydomonas reinhardtii and Arabidopsis thaliana. Biochimica et Biophysica Acta (BBA) - Bioenergetics, 2005. 1706(3): p. 267-275.

96. Adir, N., S. Bar-Zvi, and D. Harris, The amazing phycobilisome. Biochimica et Biophysica Acta (BBA) - Bioenergetics, 2020. 1861(4): p. 148047.

97. Gantt, E. and S. Conti. Phycobiliprotein localization in algae. in Brookhaven symposia in biology. 1966.

98. Gantt, E. and S. Conti, Granules associated with the chloroplast lamellae of Porphyridium cruentum. The Journal of cell biology, 1966. 29(3): p. 423-434.

99. Zhang, J., et al., Structure of phycobilisome from the red alga Griffithsia pacifica. Nature, 2017. 551 (7678): p. 57-63.

100. Zilinskas, B.A. and L.S. Greenwald, Phycobilisome structure and function. Photosynthesis Research, 1986. 10(1): p. 7-35.

101. Watanabe, M. and M. Ikeuchi, Phycobilisome: architecture of a light-harvesting supercomplex. Photosynthesis Research, 2013. 116(2): p. 265-276.

102. Adir, N., Elucidation of the molecular structures of components of the phycobilisome: reconstructing a giant. Photosynthesis Research, 2005. 85(1): p. 15-32.

103. Marx, A., L. David, and N. Adir, Piecing together the phycobilisome. The Structural Basis of Biological Energy Generation, 2014: p. 59-76.

104. Yu, M.H. and A.N. Glazer, Cyanobacterial phycobilisomes. Role of the linker polypeptides in the assembly of phycocyanin. J Biol Chem, 1982. 257(7): p. 3429-33.

105. Liu, L.-N., et al., Characterization, structure and function of linker polypeptides in phycobilisomes of cyanobacteria and red algae: An overview. Biochimica et Biophysica Acta (BBA) - Bioenergetics, 2005. 1708(2): p. 133-142. 
106. Dartnell, L., et al., Degradation of Cyanobacterial Biosignatures by Ionizing Radiation. Astrobiology, 2011. 11: p. 997-1016.

107. Harris, D., et al., The Structural Basis for the Extraordinary Energy-Transfer Capabilities of the Phycobilisome, in Membrane Protein Complexes: Structure and Function, J.R. Harris and E.J. Boekema, Editors. 2018, Springer Singapore: Singapore. p. 57-82.

108. Tamary, E., et al., Structural and functional alterations of cyanobacterial phycobilisomes induced by high-light stress. Biochimica et Biophysica Acta (BBA) - Bioenergetics, 2012. 1817(2): p. 319327.

109. Bar Eyal, L., et al., Changes in aggregation states of light-harvesting complexes as a mechanism for modulating energy transfer in desert crust cyanobacteria. Proceedings of the National Academy of Sciences, 2017. 114(35): p. 9481.

110. Piven, I., G. Ajlani, and A. Sokolenko, Phycobilisome linker proteins are phosphorylated in Synechocystis sp. PCC 6803. J Biol Chem, 2005. 280(22): p. 21667-72.

111. Chenu, A., et al., Light Adaptation in Phycobilisome Antennas: Influence on the Rod Length and Structural Arrangement. J Phys Chem B, 2017. 121(39): p. 9196-9202.

112. Porcar-Castell, A., et al., Linking chlorophyll a fluorescence to photosynthesis for remote sensing applications: mechanisms and challenges. Journal of Experimental Botany, 2014. 65(15): p. 4065-4095.

113. Campbell, D., et al., Chlorophyll fluorescence analysis of cyanobacterial photosynthesis and acclimation. Microbiology and molecular biology reviews : MMBR, 1998. 62(3): p. 667-683.

114. Schreiber, U., Pulse-Amplitude-Modulation (PAM) Fluorometry and Saturation Pulse Method: An Overview, in Chlorophyll a Fluorescence: A Signature of Photosynthesis, G.C. Papageorgiou and Govindjee, Editors. 2004, Springer Netherlands: Dordrecht. p. 279-319.

115. Suggett, D.J., et al., Fast repetition rate and pulse amplitude modulation chlorophyll a fluorescence measurements for assessment of photosynthetic electron transport in marine phytoplankton. European Journal of Phycology, 2003. 38(4): p. 371-384.

116. Ruban, A.V., Nonphotochemical Chlorophyll Fluorescence Quenching: Mechanism and Effectiveness in Protecting Plants from Photodamage. Plant physiology, 2016. 170(4): p. 19031916.

117. Tian, L., et al., pH dependence, kinetics and light-harvesting regulation of nonphotochemical quenching in \&lt;em\&gt;Chlamydomonas\&lt;/em\&gt. Proceedings of the National Academy of Sciences, 2019. 116(17): p. 8320.

118. Van Stokkum, I.H.M., et al., (Sub)-Picosecond Spectral Evolution of Fluorescence Studied with a Synchroscan Streak-Camera System and Target Analysis, in Biophysical Techniques in Photosynthesis, T.J. Aartsma and J. Matysik, Editors. 2008, Springer Netherlands: Dordrecht. p. 223-240.

119. Hirvonen, L.M. and K. Suhling, Fast Timing Techniques in FLIM Applications. Frontiers in Physics, 2020. 8(161). 
CHAPTER

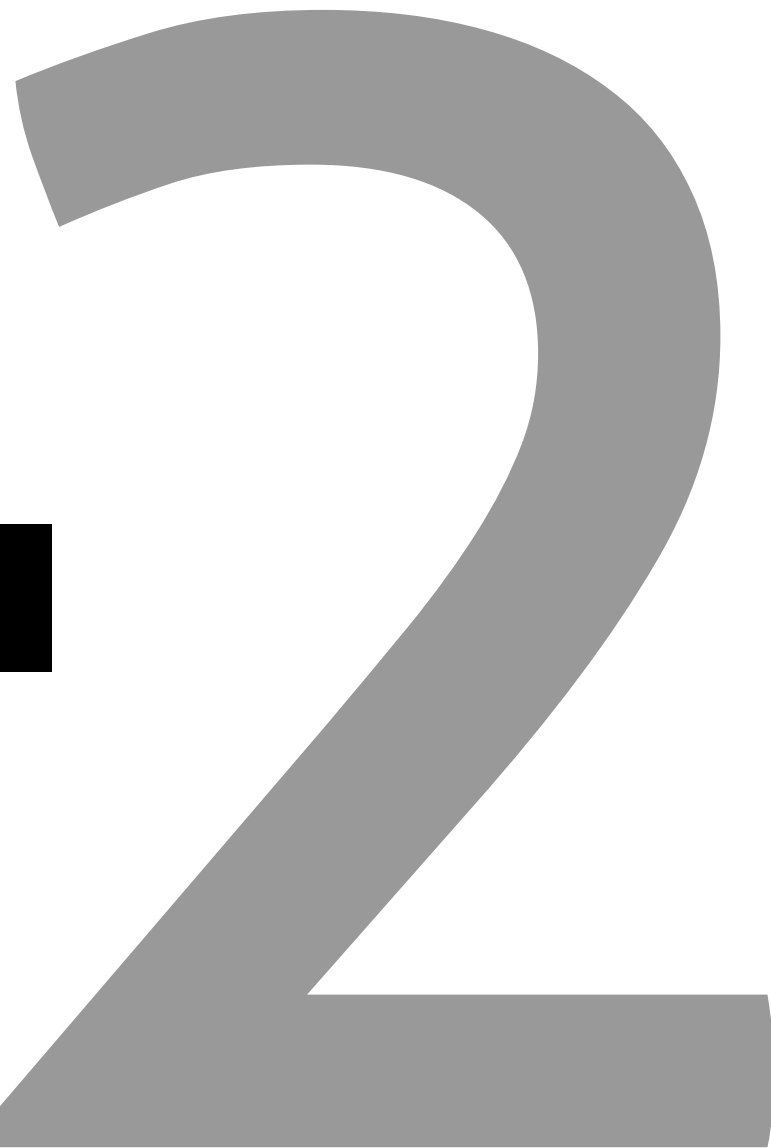




\title{
State transitions in cyanobacteria studied with picosecond fluorescence at room temperature
}

\author{
Ahmad Farhan Bhatti, Reza Ranjbar Choubeh, Diana Kirilovsky, \\ Emilie Wientjes, Herbert van Amerongen
}


Cyanobacteria can rapidly regulate the relative activity of their photosynthetic complexes photosystem I and II (PSI and PSII) in response to changes in the illumination conditions. This process is known as state transitions. If PSI is preferentially excited, they go to state I whereas state II is induced either after preferential excitation of PSII or after dark adaptation. Different underlying mechanisms have been proposed in literature, in particular i) reversible shuttling of the external antenna complexes, the phycobilisomes, between PSI and PSII, ii) reversible spillover of excitation energy from PSII to PSI and iii) a combination of both, iv) increased excited-state quenching of the PSII core in state II. Here we investigated wild-type and mutant strains of Synechococcus sp. PCC 7942 and Synechocystis sp. PCC 6803 using timeresolved fluorescence spectroscopy at room temperature. Our observations support model iv, meaning that increased excited-state quenching of the PSII core occurs in state II thereby balancing the photochemistry of photosystems I and II. 


\section{Introduction}

In photosynthetic organisms harvesting of sunlight is the initial step in the process of converting solar energy to chemical energy ${ }^{[1,2]}$. Photon absorption in cyanobacteria takes place in membrane-external pigment-protein antenna complexes (phycobilisomes) and membrane-embedded chlorophyll (Chl) $a$ antenna complexes ${ }^{[3]}$. Harvested photon energy is transferred to the reaction centre complexes of photosystems I and II (PSI and PSII) through a series of (down-hill) excitation-energy transfer (EET) steps ${ }^{[4]}$. The amount of excitation energy arriving at the reaction centre of a photosystem is proportional to the absorption cross section of the associated antenna complexes and the efficiency of the EET process.

Cyanobacteria can rapidly regulate the relative activity of PSI and PSII in response to changes in the illumination conditions ${ }^{[5]}$ including dark-light transitions ${ }^{[6,7]}$. This regulatory process for optimal photosynthesis under low-light conditions is known as state transitions ${ }^{[2,8-10]}$. The trigger for state transitions is the redox state of the plastoquinone pool between PSI \& PSII ${ }^{[6]}$. Oxidation of the plastoquinone pool by light which preferentially excites PSI induces a transition to state I whereas reduction of the plastoquinone pool by the preferential excitation of PSII induces a transition to state II. State II can also be induced by respiratory electron flow into the plastoquinone pool in the dark ${ }^{[6]}$. State transitions are characterized by a relative decrease of PSII fluorescence in state II as compared to state $1^{[11]}$.

In green plants, the redistribution of light-harvesting complex II (LHCII) between PSII and PSI is part of the physiological mechanism accompanying state transitions ${ }^{[12-14]}$. Despite the discovery of state transitions in cyanobacteria long ago ${ }^{[9]}$, a comprehensive understanding of the process is still evasive. The phycobilisome (PBS) antenna in cyanobacteria is the functional equivalent of the LHCII antenna in plants. The (re)distribution of the excitation energy to the photosystems through reversible migration of PBSs between PSI and PSII during state transitions, was proposed in some earlier studies ${ }^{[15-18]}$. Rapid diffusion of the PBSs along the surface of thylakoid membranes leading to transient interactions (association/disassociation) with the less mobile photosystems is at the basis of the mobile PBS model ${ }^{[19]}$. However, the PBS mobility model fails to explain the change in ratio of PSI/PSII fluorescence upon $\mathrm{Chl} a$ excitation ${ }^{[20]}$. Another model for state transitions in cyanobacteria, the one of direct "spillover" of excitation energy from PSII to PSI was proposed earlier for Anacystis nidulans ${ }^{[21]}$ and for Synechocystis PCC $6803^{[22]}$ to explain the fluorescence changes during state transitions upon $\mathrm{Chl} a$ excitation. According to the spillover model, the excess excitation energy available at PSII in state II (absorbed by the PBS antenna or Chl $a$ inner antenna of PSII) spills over from PSII to PSI. The spillover model was supported by the observation that in state I the PSII complexes are arranged in a row while in state II the arrangement of PSII complexes becomes more random, which may 
result in a decreased distance between PSII and PSI, thereby facilitating direct excitation energy transfer (EET) from PSII to PSI ${ }^{[23]}$. A functional megacomplex consisting of PBSPSII-PSI was isolated from Synechocystis PCC $6803^{[24]}$ and was considered to support this spillover model. Time-resolved fluorescence characterization of the megacomplex revealed that one PBS could transfer the excitation energy to both photosystems and energy transfer to PSI was found to be less efficient than to PSII ${ }^{[24]}$, although direct evidence for this spillover was not provided. In addition, the evidence for the presence of megacomplexes in intact cells and their characterization in state transitions is to date not available ${ }^{[7]}$. Incompleteness of the spillover model became explicit in a number of studies in which fluorescence changes, characteristic for state transitions, were less pronounced upon $\mathrm{Chl} a$ excitation as compared to PBS excitation ${ }^{[20]}$. To account for these differences, a comprehensive model (mixed model), combining the features of PBS mobility and spillover was proposed for Synechococcus PCC $7942^{[20,25] .}$

In the cyanobacteria Synechocystis PCC 6803, Synechococcus PCC 7002, and Anabaena PCC 7120, mutants lacking the ApcD gene product were reported to be impaired in performing state transitions ${ }^{[26-29]}$. Emlyn-Jones et al. ${ }^{[30]}$ observed that the Synechocystis $s p$. PCC $6803 \mathrm{rpaC}^{-}$mutant did not show any fluorescence changes related to state transitions upon PBS excitation although they were observed upon $\mathrm{Chl} a$ excitation. This observation clearly revealed that both a spillover as well as a combination model are insufficient to fully describe the presence/absence of fluorescence changes during state transitions. McConnell et al. ${ }^{[20]}$ put forward a modified version of the spillover model to account for this experimental observation. They suggested that excitations created in the PBSs are directly transferred to the PSII reaction centres and cannot be lost via spillover to PSI in state II. In the wild-type cyanobacteria, the rate of EET from PBS to PSI was proposed to increase in state II, whereas it did not increase in the Synechocystis rpaC- ${ }^{-}$and Synechococcus 7002 ApcD mutants ${ }^{[20]}$. On the other hand, it was proposed that excitations in the core antenna complexes (CP43 and CP47) of PSII can spill over to PSI in state II, both in the wild-type and mutants. However, experimental evidence for changes in EET from PSII to PSI and from PBS to PSI in state II during state transitions is lacking so far ${ }^{[7,31]}$. McConnell et al. ${ }^{[20]}$ concluded that their model, which suggests only slight changes in the association of the PBS-PSII supercomplex with PSI is not compatible with the FRAP data, which indicates a higher lateral mobility of the PBSs as compared to the PSIl-associated $\mathrm{Chl} a$ molecules after photobleaching with a laser flash ${ }^{[19]}$. In a 77K time-resolved fluorescence study of state transitions in the cyanobacterium Synechococcus elongatus no evidence was found neither for the migration of PBSs from PSII to PSI in state II nor for the spillover of energy from PSII to PSI ${ }^{[31]}$ and both processes were ruled out [31]. Instead the observed decrease in PSII fluorescence in state II was ascribed to direct quenching of PSII complexes ${ }^{[31]}$. 
Which model or combination of models presented above, would best describe the state transitions in cyanobacteria at physiologically relevant conditions? Is there some common explanation for all or most of the experimental observations reported in literature regarding state transitions in cyanobacteria? Do PBSs and PSIl complexes have independent roles during state transitions? Is the PBS core involved in the physical mechanism required for the state transitions in cyanobacteria?

To find answers to these questions we investigated strains of the cyanobacteria Synechococcus sp. PCC 7942 (hereafter Synechococcus) and Synechocystis sp. PCC 6803 (hereafter Synechocystis) using time-resolved fluorescence spectroscopy. Light harvesting in cyanobacteria is primarily accomplished by the PBS antenna ${ }^{[22-34]}$. Structurally, a PBS is composed of fan-like phycocyanin (PC) rods which radiate from a central core. The core attaches to the stromal side of the photosynthetic membrane and is comprised of cylinders (number of cylinders is specific to the organism) which are composed of trimeric discs of allophycocyanin (APC) ${ }^{[34]}$. In Synechococcus and Synechocystis the PBS core is comprised of bi-and tri-cylindrical APC trimeric discs, respectively. In the case of Synechocystis only two basal cylinders of the core bind to the thylakoid membrane ${ }^{[29]}$. Three important components of this core are the phycobiliproteins ApcD, ApcE and ApcF. ApcD and ApcE are the final acceptors of PBS-absorbed excitation energy, meaning that their lowest excited states are lower in energy than those of the other pigments in the PBS. ApcF on the other hand influences the optical properties of ApcE and could modify the energy transfer to one or both photosystems depending on the strain ${ }^{[27,28,33]}$. These three components of the PBS core transfer the PBS-absorbed excitation energy to the photosystems ${ }^{[28,29]}$. To characterize the role of the PBS core and its low-energy pigments during state transitions, the mutants lacking the ApcD and ApcF components in both strains were also studied. The lack of ApcD and ApcF was concluded to inhibit/decrease state transitions in Synechocystis ${ }^{[28,29]}$ but not in S. elongatus. A mutant lacking ApcE component was not used in this work, because the PBSs are no longer stably attached; the ApcE subunit, also known as $L_{C M}$ is essential for the PBS assembly and is also a structural component of the PBS core and involved in thylakoid membrane interaction ${ }^{[29,35-37]}$. All of the experiments in the present study were performed at room temperature (RT) and states I and II were induced by keeping the cells in blue light and darkness, respectively.

\section{Materials and Methods}

\subsection{Strains and Growth Conditions}

Synechocystis and Synechococcus, WT, $\triangle \mathrm{ApcD}$ and $\triangle \mathrm{ApcF}$ strains were grown in BG11 medium ( $20 \mathrm{ml} /$ liter) buffered with sodium bicarbonate $(0.85 \mathrm{~g} /$ liter), and sodium nitrate (1.75 g/liter) at $\mathrm{pH} \sim 8.0$. Medium for mutants deficient in $\triangle \mathrm{ApcF}$ was complemented with spectinomycin $(25 \mu \mathrm{g} / \mathrm{ml})$ and streptomycin $(10 \mu \mathrm{g} / \mathrm{ml})$. Media for Synechocystis $\Delta$ ApcD 
and Synechococcus $\triangle \mathrm{ApcD}$ were complemented with chloramphenicol $(20 \mu \mathrm{g} / \mathrm{ml})$ and kanamycin $(40 \mu \mathrm{g} / \mathrm{ml})$, respectively. All strains were grown at $30^{\circ} \mathrm{C}$ under white light illumination at $50 \mu \mathrm{mol}$ photons $\mathrm{m}^{-2} \mathrm{~s}^{-1}$ in $250 \mathrm{ml}$ flasks shaken at $100 \mathrm{rpm}$, containing culture volumes of $60 \mathrm{ml}$ as described in ${ }^{[31]}$. The construction of Synechocystis $\triangle A p c D$ and $\triangle \mathrm{ApcF}$ was described in ${ }^{[35]}$ and that of Synechococcus $\triangle \mathrm{ApcD}$ and $\triangle \mathrm{ApcF}$ in ${ }^{[29]}$.

\subsection{Sample Preparation}

All the WT and mutant cells were harvested during their logarithmic growth phase at optical density $\sim 0.6-0.7$ at $800 \mathrm{~nm}\left(O D_{800}\right.$ for a path length of $1 \mathrm{~cm}$. Cells were diluted with their respective fresh medium to $O D_{630} \sim 0.35$ as measured with a Cary 4000 UV-Vis spectrophotometer with integrating sphere. To drive the cells to state I and II, they were kept in blue light (of $452 \pm 40 \mathrm{~nm}$ at $\sim 40 \mu \mathrm{mol}$ photons $\mathrm{m}^{-2} \mathrm{~s}^{-1}$ intensity) and darkness, respectively, for about 15 minutes. Using a pulse-amplitude modulated fluorometer (PAM101; Walz, Effeltrich, Germany) state transitions were monitored. Measurements were performed in a $1 \mathrm{~cm} \times 1 \mathrm{~cm}$ stirred quartz cuvette.

\subsection{Replicates}

All time-resolved fluorescence experiments were performed at least twice on each individual sample on the same day (technical replicates) while biologically independent experiments (biological replicates) were performed on different samples on different days separated by a few weeks up to several months. Representative data from each strain is presented in this work and all changes/differences that are relevant for our conclusions are fully reproducible. Standard deviations are given for the decrease in PSII fluorescence upon State I to State II transition.

\subsection{Time-resolved fluorescence spectroscopy}

Time-resolved fluorescence measurements were performed at room temperature with a synchro scan streak camera ${ }^{[38]}$ as described in ${ }^{[7,31]}$. Sub-picosecond pulses of $577 \mathrm{~nm}$ and $430 \mathrm{~nm}$ wavelength with repetition rate of $3.8 \mathrm{MHz}$ were used to preferentially excite the PBSs and photosystems, respectively. The laser power was $\sim 20 \mu \mathrm{W}$ for PBS excitation and $\sim 60 \mu \mathrm{W}$ for $\mathrm{Chl} a$ excitation. The laser spot size was $\sim 0.1 \mathrm{~mm}$ in both cases. Timeresolved emission spectra were recorded with a time window of 800 ps for resolving the fast components and 2000 ps for estimation of the slowest component. Sixty streak images were recorded, where each image was an analogue integration of 20 exposures with exposure time of 1112 ms each. Streak images were corrected for background and for wavelength-dependent sensitivity of the detector. Processed streak images were then globally analysed using the Glotaran ${ }^{[39]}$ and TIMP package for $\mathrm{R}^{\left[{ }^{[0]}\right.}$ to determine the fluorescence lifetimes and decay-associated spectra (DAS) as described in [7, 31]. For all of our measurements the time-resolved fluorescence is described with five components after $577 \mathrm{~nm}$ excitation and four components after $430 \mathrm{~nm}$ excitation. For each measurement 
the obtained DAS were summed, which provided the estimated fluorescence spectrum at $\mathrm{t}=0$, i.e. immediately upon excitation and this $\mathrm{t}=0$ spectrum was normalized to a value of 1 in the maximum. All the individual DAS were corrected with the same scaling factor. Steady-state fluorescence spectra were subsequently calculated as a sum of the products of amplitudes (DAS) and their respective decay times and below they are referred to as reconstructed (steady-state) spectra. For all streak experiments cells adapted to state I were kept under the same light condition for the duration of the measurement. To verify the reversibility of the state transitions in our samples, cells measured in state I were adapted first to darkness for $\sim 15$ minutes and then again measured in state I. Timeresolved emission of both state I measurements were compared and were found to be virtually identical. Measurements were performed in a $1 \mathrm{~cm} \times 1 \mathrm{~cm}$ stirred quartz cuvette.

\section{Results}

\subsection{Pulse-amplitude modulated fluorometry}

In cyanobacteria, state transitions balance the relative amount of PSI and PSII excitations, which is reflected in the (relative) amount of PSI and PSII fluorescence. Therefore, state transitions can readily be monitored by measuring the level of PSI and PSII fluorescence at $77 \mathrm{~K}{ }^{[41-44]}$. At room temperature the fluorescence decay of PSI is extremely fast $(\sim 20$ ps) and therefore has a very small contribution to the steady-state fluorescence which is dominated by PSII emission ${ }^{[45]}$.

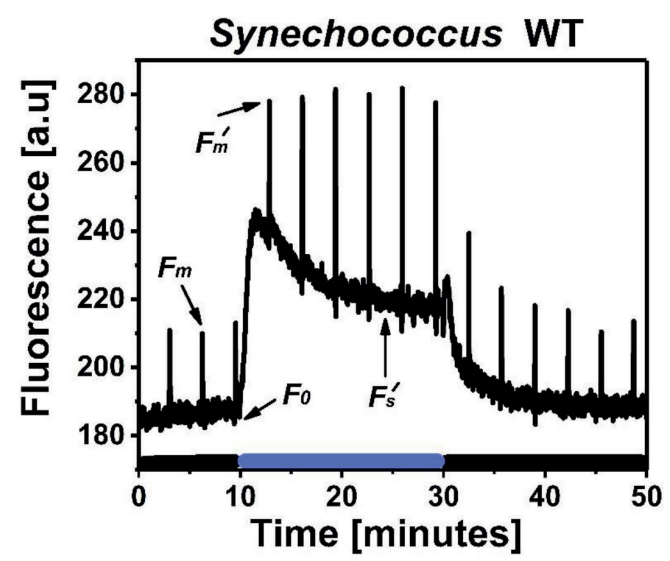

Figure 1. Time traces of variable fluorescence in wild-type Synechococcus 7942 measured with the pulseamplitude modulated fluorescence technique. The fluorescence detection window was $>700 \mathrm{~nm}$. Black and blue thick solid lines along the time-axis represent the time periods of cells in darkness and blue light respectively. Blue light with central wavelength $452 \mathrm{~nm}$ and $\sim 40 \mu \mathrm{mol}$ photons $\mathrm{m}^{-2} \mathrm{~s}^{-1}$ was used to bring the cells to state I. Blue-light-adapted cells were subsequently put in darkness to bring the cells to state II. $F_{0}, F_{m}$ represent minimum and maximum fluorescence, respectively, for dark-adapted cells. $F_{S}$ and $F_{m}$ are steady-state and maximum fluorescence, respectively, for cells in blue light. 
To estimate the time it takes to induce state I and state II in cyanobacteria, use was made of pulse-amplitude modulated (PAM) fluorometry. With this method, state transitions in photosynthetic organisms can be followed at room temperature, since they are characterized by changes in the levels of PSII fluorescence as a function of the intensity and spectrum of the actinic light, which is used to drive photosynthesis ${ }^{[46]}$. In Figure 1 a fluorescence time trace is shown for Synechococcus WT cells in different illumination conditions as recorded with a PAM fluorometer.

The PAM fluorescence trace contains some contribution from free phycobilisomes (PBS) and PSI but the major contribution stems from PSII and PBS complexes connected to the photosystems ${ }^{[47]} . F_{0}$ and $F_{S}$ represent the minimum fluorescence for cells kept in darkness and the steady-state fluorescence in the (blue) light, respectively. Minimum fluorescence was measured with non-actinic $1 \mu \mathrm{sec}$ pulses of measuring light with 1.6 $\mathrm{kHz}$ frequency. The minimum fluorescence represents the fluorescence of cells with mainly open reaction centres. Periodic saturating pulses of white light closed the PSII RCs, causing the fluorescence to reach the maximum level, i.e. $F_{m}$ for cells in darkness and $F_{m}$, for cells in the light. The extent of state transitions is proportional to the relative change in the maximum fluorescence.

A sharp rise in fluorescence for $F_{0}, F_{S}$ ' and $F_{m}$ levels was seen when dark-adapted cells were exposed to blue light to bring them into state I. A decrease was observed when light-adapted cells were again put in darkness to drive the cells to state II. Periods of 20 minutes were found to be sufficient to bring the majority of the cells to state I/II from state II/I, respectively.

\subsection{Time-resolved fluorescence spectroscopy results}

Use was made of the time-resolved fluorescence spectroscopy to study the state transitions in both wild-type species of Synechococcus, Synechocystis and their $\Delta A p c D$ and $\Delta$ ApcF mutants.

In time-resolved fluorescence spectroscopy an accurate interpretation of the fluorescence decay lifetimes and their associated wavelength-dependent amplitudes is crucial for the understanding of the phenomena under investigation. To be sure that the fluorescence changes observed in our measurements are represantative of state transitions only, all the measurements were done at the $F_{m}$ (closed PSII reaction centres) level of the cells. Figure 2 shows the fluorescence decay traces at $\sim 680 \mathrm{~nm}$ (PSII and PBS emission wavelengths) for Synechococcus cells, both in state I and state II at various powers of the $577 \mathrm{~nm}$ excitation pulses. The fluorescence decay traces measured with weak-intensity pulses (average laser output $0.1 \mu \mathrm{W}-0.15 \mu \mathrm{W}$ (black curves)) correspond to the situation in which the PSII reaction centres were predominantly open. 


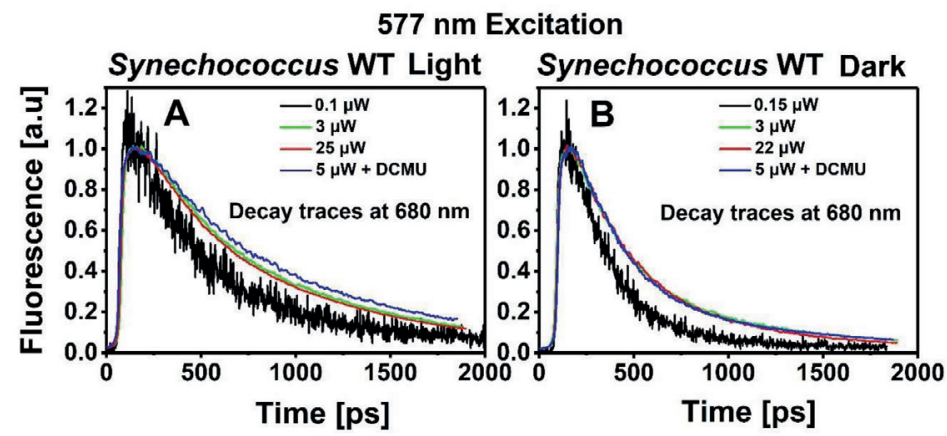

Figure 2. Fluorescence decay traces of Synechococcus cells at $680 \mathrm{~nm}$ obtained with various laser powers after $577 \mathrm{~nm}$ excitation (left) light-adapted cells (state I) and (right) dark-adapted cells (state II). Black, green and red traces correspond to cells without DCMU and the blue trace to DCMU-treated cells. All traces were normalized to each other at their maximum emission. Measurements were performed at room temperature.

Raising the power to $3 \mu \mathrm{W}$ led to fluorescence with closed PSIl reaction centres. Increasing the power further $(22 \mu \mathrm{W}-25 \mu \mathrm{W})$ did not cause an additional slowing down of the kinetics and the kinetics were similar/identical to those with the reaction centres closed by the use of DCMU. The similarity of the fluorescence decay kinetics in the range of 3-25 $\mu \mathrm{W}$ also demonstrates that the fluorescence kinetics were not affected by singlet-singlet or singlet-triplet annihilation which would have shortened the excited-state lifetime at higher excitation powers ${ }^{[46]}$.

In Figure 3 characteristic fluorescence decay traces at the maximum emission ( 680 nm) of PBS terminal emitters and PSII, for Synechococcus and Synechocystis cells in state I and II are shown upon $577 \mathrm{~nm}$ excitation. The fluorescence kinetics at $680 \mathrm{~nm}$ show a slower decay for the cells in state I as compared to the cells in state II for both strains, which is characteristic for state transitions in cyanocteria. The fluorescence changes are much more pronounced for Synechococcus (Figure 3A) than for Synechocystis (Figure 3B).

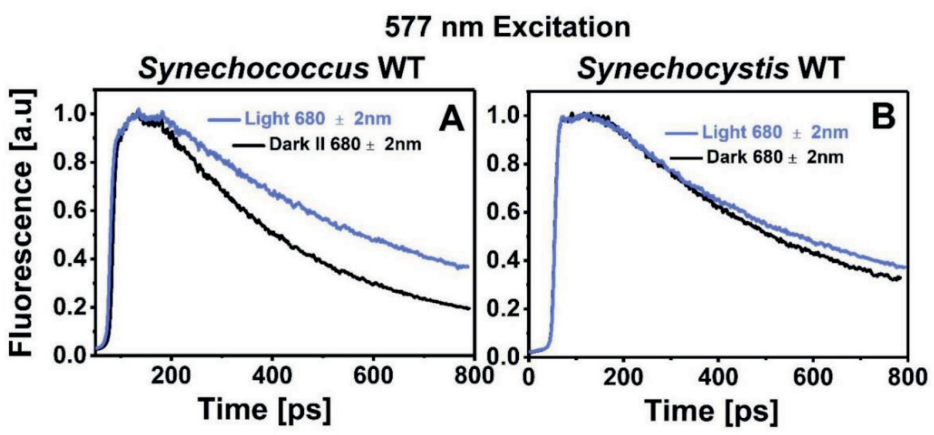

Figure 3. Normalized decay traces at $680 \mathrm{~nm}$ (PBS terminal emitter $\left(A P C_{680}\right)$ and PSII) for dark-adapted (black lines) and light-adapted cells (blue lines) of (A) Synechococcus and (B) Synechocystis. Excitation wavelength was $577 \mathrm{~nm}$ and measurements were done at room temperature. 


\subsection{State transitions in Synechococcus WT, $\triangle A p c D$ and $\triangle A p c F$}

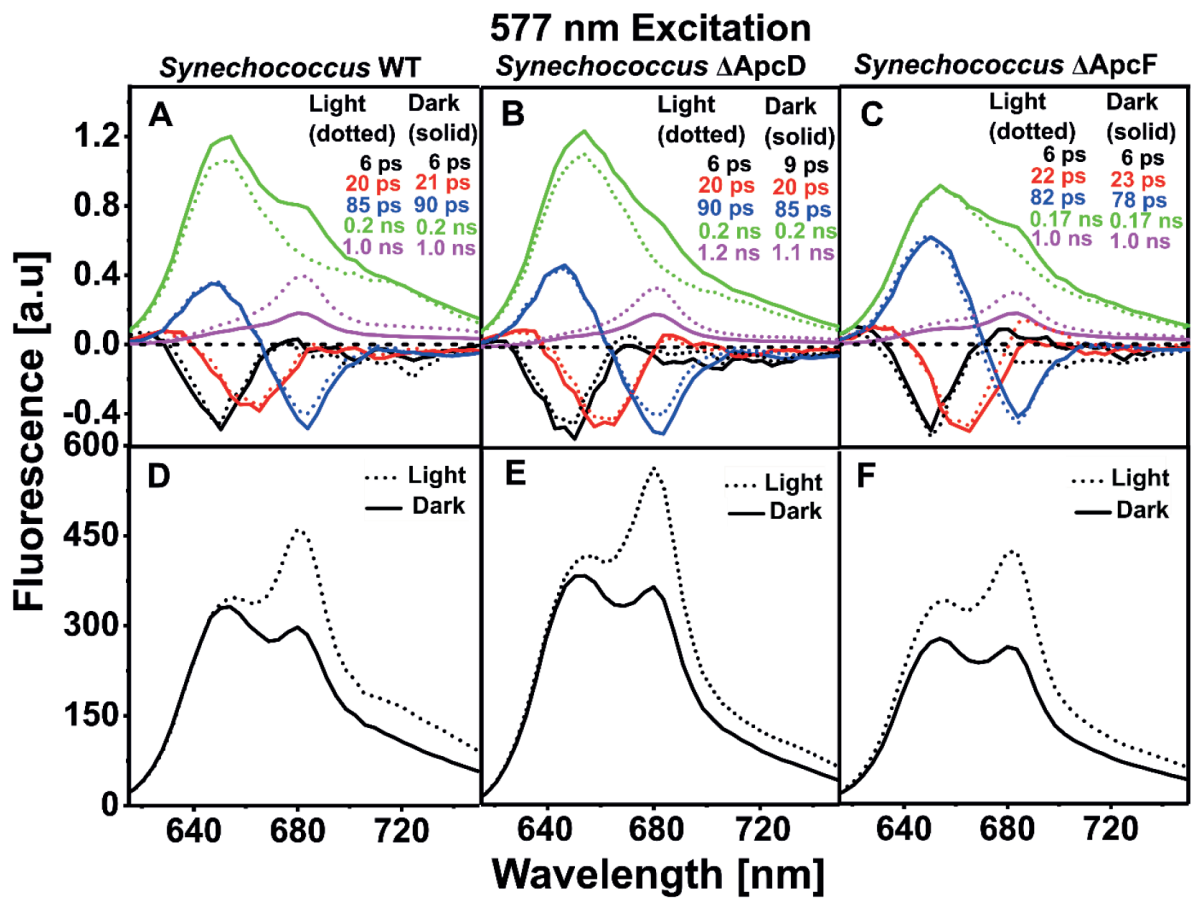

Figure 4. Decay-associated spectra obtained from global fitting of time-resolved fluorescence results after 577 $n m$ excitation. $(A, B, C)$ five-components DAS of Synechococcus WT, Synechococcus $\triangle A p c D$ and Synechococcus $\triangle A p c F$, in state I (dotted lines) and state II (solid lines). DAS-associated lifetimes are presented in the insets with corresponding colours. In $A$ and $C$ the slowest lifetime is fixed to $1.0 \mathrm{~ns}$. (D,E,F) reconstructed steady-state spectra for Synechococcus WT, Synechococcus $\triangle \mathrm{ApcD}$ and Synechococcus $\triangle \mathrm{ApcF}$, respectively, in state I (dotted line) and state II (solid line). All spectra are scaled as described in Materials and Methods.

Measurements were performed both with selective excitation of the PBSs (577 nm excitation pulses) and the photosystems (430 nm excitation pulses) to determine the individual roles of the phycobilisomes and photosystems during state transitions. The recorded time-resolved fluorescence spectra in each case were analysed with the globalanalysis method to obtain the DAS.

Five-components DAS for WT, $\triangle \mathrm{ApcD}$, and $\triangle \mathrm{ApcF}$ Synechococcus cells are shown in Figure 4 for cells in state I (dotted lines) and II (solid lines). The $\leq 10$ ps DAS with negative peak at $650 \mathrm{~nm}$ reflect EET within phycocyanin (PC) discs ${ }^{[31,48,49]}$. EET occurs predominantly from higher-energy to lower-energy pigments. The $\sim 20$ ps DAS with positive peak at $630 \mathrm{~nm}$ and negative peak at $660 \mathrm{~nm}$ describe down-hill EET from PC rods to the $A P C_{660}$ core. The 80-90 ps DAS describe EET from higher-energy pigments in the PC rods ( $655 \mathrm{~nm}$ peak) to the lower-energy pigments in $A P C_{680}(\sim 680 \mathrm{~nm})$ and Chls. The first three DAS are very similar for each type of cells in state I and II and demonstrate that EET within PBSs is not substantially affected during state transitions. 
Transitioning of the WT and mutant cells from state I to state II (light to dark) was accompanied with: (i) a decrease in the amplitude of the (slowest) $\tau=\sim 1.0$ ns component and (ii) a concomitant rise of the amplitude of the $\tau=\sim 0.17-0.2 \mathrm{~ns}$ component at $680 \mathrm{~nm}$. In an earlier study of Synechococcus 6301 the $\sim 0.15$ ns component with the emission band around $\sim 680 \mathrm{~nm}$ (corresponding to $\sim 0.17-0.2 \mathrm{~ns}$ component in the present work) was assigned to the PBS terminal emitters ${ }^{[44]}$.

However, in our present work we also observe this $\sim 0.2 \mathrm{~ns}$ component with emission band around $680 \mathrm{~nm}$ upon selective excitation of $\mathrm{Chl} a$ (Figures 5 and 7), strongly suggesting that it originates from PSII. Tian et al. in ${ }^{[45]}$ probed the picosecond fluorescence kinetics of the PSIl complexes (with closed reaction centres) using the PAL (PBS-less) mutant of Synechocystis 6803 . Similar kinetic components ( $\sim .2 \mathrm{~ns}$ and $\sim 1.0 \mathrm{~ns})$ were resolved in their work and show that the PSII fluorescence decay in cyanobacteria is inherently heterogenous (biphasic/multiphasic) and is not the result of a mixed status of the reaction centres (partially closed). However, upon PBS excitation, the presence of this particular $\sim 0.2 \mathrm{~ns}$ decay component with its maximum emission at $680 \mathrm{~nm}$ is not readily observable in the five component global analysis of the time-resolved fluorescence of light-adapted (state I) cells (Figure 4). It overlaps with the $\sim 0.17-0.2$ ns DAS in state I (Figure 4A) with a broad positive peak at $\sim 655 \mathrm{~nm}$ and a depression around $\sim 680 \mathrm{~nm}$ which is due to EET from phycocyanins and allophycocyanins to the photosystems. This makes it difficult to reliably separate them in our global analysis. The $\sim 1.0 \mathrm{~ns}$ DAS with the maximum emission around $\sim 680 \mathrm{~nm}$ is assigned to the PSIl complexes with closed reaction centres and PBS terminal emitters (free and partially connected PBSs). From now on we will mainly discuss the $\sim 0.17-0.2 \mathrm{~ns}$ and $\sim 1.0 \mathrm{~ns}$ components with their emission maxima at $\sim 680 \mathrm{~nm}$ and refer to them as the slow ( 0.17-0.2 ns) and the slowest ( $1.0 \mathrm{~ns})$ component. The value of the lifetime of the slowest component is not extremely accurate and was found to be in the range of $\sim 0.8-1.0$ ns. Cells in state II had consistently slightly higher values for the slowest component (Figure S2) as observed in ${ }^{[44]}$.

Reconstructed steady-state fluorescence spectra of Synechococcus WT, $\triangle A p c D$ and, $\triangle \mathrm{ApcF}$ mutants in Figure 4 show a $\sim 35.8 \pm 2.5 \%, 35.0 \pm 0.1 \%$ and, $39.4 \pm 4.9 \%$ decrease of PSII fluorescence in state II, respectively. The ApcD and ApcF components are therefore concluded not to be required for the state transitions in Synechococcus, in agreement with the conclusions in ${ }^{[29]}$.

Figure 5 presents the four DAS components for Synechococcus WT and the $\triangle A p c D$ and, $\triangle \mathrm{ApcF}$ mutants, obtained upon selective $\mathrm{Chl} a$ excitation at $430 \mathrm{~nm}$. Due to the higher $\mathrm{Chl} a$ content in PSI, the fluorescence kinetics are dominated by the $\sim 20 \mathrm{ps}$ component $(\lambda$ $=690 \mathrm{~nm}$ ), representing the kinetics of PSI excitation trapping. The fast component ( 68 ps) with a broad positive peak around $680-690 \mathrm{~nm}$ corresponds to excitation-energy 
equilibration in the bulk chlorophylls of PSI and PSII. The negative peaks in the same DAS around $\sim 645 \mathrm{~nm}$ and above $700 \mathrm{~nm}$ indicate EET from the bulk Chls to the PBSs and to the lower-energy Chls, respectively.

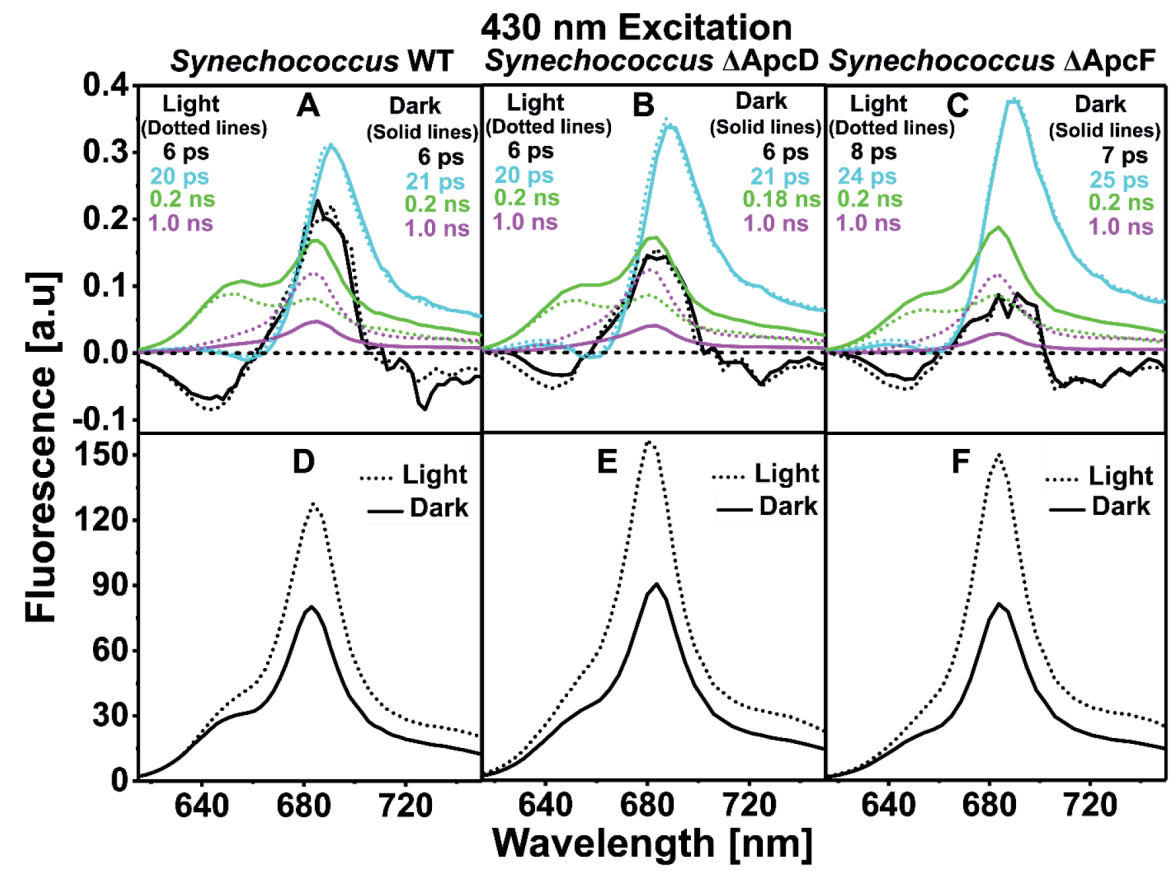

Figure 5. Decay-associated spectra (DAS) obtained from global fitting of time-resolved fluorescence results upon $430 \mathrm{~nm}$ excitation. $(\mathrm{A}, \mathrm{B}, \mathrm{C})$ four-components DAS of Synechococcus WT, Synechococcus $\triangle \mathrm{ApcD}$ and Synechococcus $\triangle A p c F$, respectively in state I (dotted lines) and state II (solid lines). The slowest lifetime in each case was fixed to 1.0 ns. The $\sim 20$ ps DAS in each case is multiplied with 0.5 to make the two slowest DAS better visible. DASassociated lifetimes are shown in the legend with corresponding colours. $(D, E, F)$ reconstructed steady-state spectra for Synechococcus WT, Synechococcus $\triangle$ ApcD and Synechococcus $\Delta$ ApcF, respectively in state I (dotted line) and state II (solid line). All spectra are scaled as described in Materials and Methods.

Excitation at $430 \mathrm{~nm}$ is at the absorption maximum of $\mathrm{Chl} a$, where only negligible absorption is due to PBSs ${ }^{[31]}$. Therefore, the slow component $(\sim 0.2 \mathrm{~ns})$ with a band around $680 \mathrm{~nm}$ is assigned to the PSII complexes. Similar to the $577 \mathrm{~nm}$ excitation case (Figure 4), reciprocal differences in amplitudes of the slow and the slowest components are observed for cells in state I and state II, both for the WT and mutant cells (Figure 5A-C). The value of the slowest component upon $430 \mathrm{~nm}$ excitation was typically found between $\sim 0.7 \mathrm{~ns}-1.0$ ns. Reconstructed steady-state emission spectra for Synechococcus WT, $\triangle \mathrm{ApcD}$, and $\triangle \mathrm{ApcF}$ (Figure 5D-F) show a pronounced decrease of the room-temperature PSII fluorescence in state II, as was observed before at $77 \mathrm{~K}{ }^{[29,31]}$. From the reconstructed steady-state fluorescence spectra, the fluorescence decrease in state II was found to be $39.6 \pm 1.1 \%$, $42.7 \pm 1.2 \%$ and $47.3 \pm 1.1 \%$ for Synechococcus $W T, \triangle A p c D$, and $\Delta A p c F$, respectively. 


\subsection{State transitions in Synechocystis WT, $\triangle A p c D$ and $\triangle A p c F$}

In this section, state transitions in Synechocystis WT and its $\triangle \mathrm{ApcD}$ and $\triangle \mathrm{ApcF}$ mutants are compared to those of Synechococcus as described above, regarding their fluorescence properties. It turns out that the overall kinetics are rather similar for Synechococcus and Synechocystis.

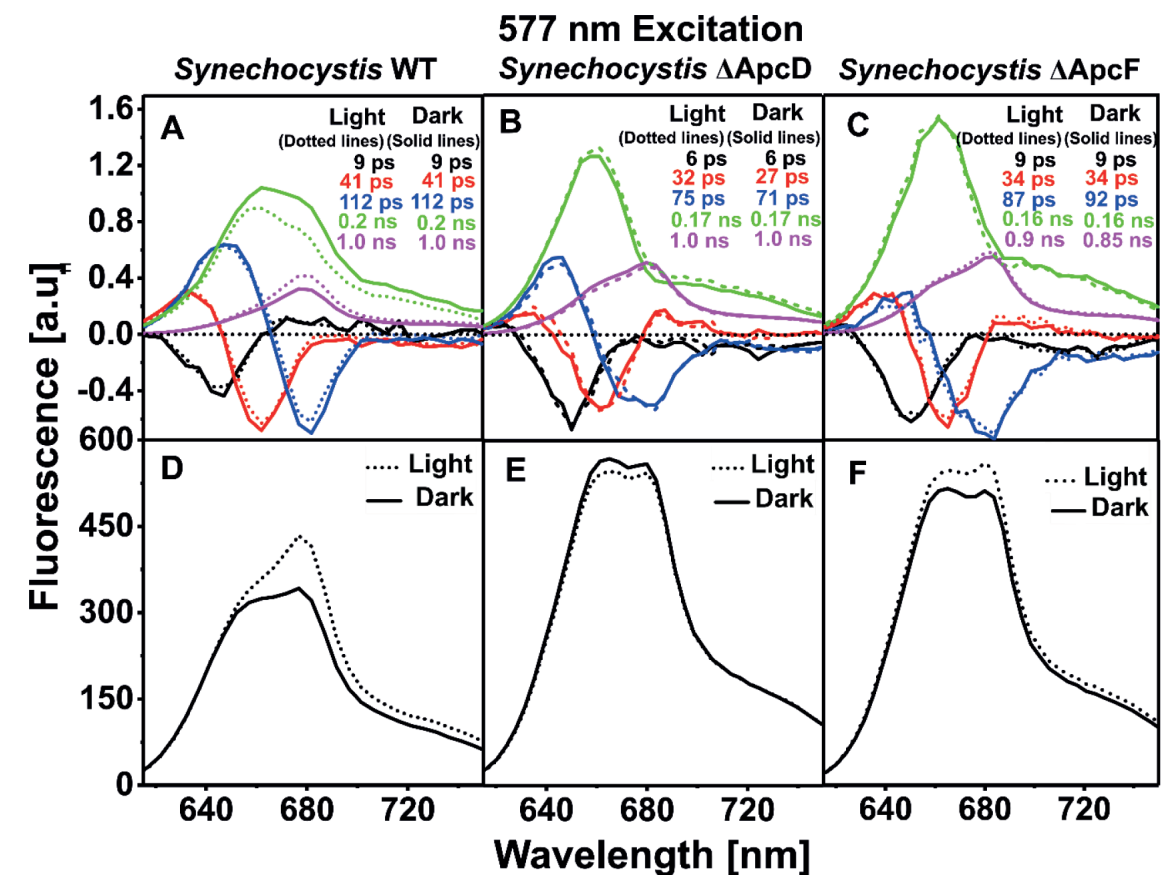

Figure 6. $(A, B, C)$ five-components DAS of Synechocystis WT, Synechocystis $\triangle A p c D$ and Synechocystis $\Delta$ ApcF, respectively in state I (dotted lines) and state II (solid lines). DAS-associated lifetimes are presented at the top with corresponding colours. In $A$ and $B$ the slowest lifetime was fixed to $1.0 \mathrm{~ns}$. (D,E,F) reconstructed steady-state spectra for Synechococcus WT, Synechococcus $\triangle A p c D$ and Synechococcus $\triangle A p c F$, respectively in state I (dotted lines) and state II (solid lines). Excitation was at $577 \mathrm{~nm}$.

Upon $577 \mathrm{~nm}$ excitation, state II is again characterized by a decrease in amplitude of the slowest component and a concomitant rise of the amplitude of the slow component (Figure $6 \mathrm{~A})$. However, these characteristic changes are completely absent in Synechocystis $\triangle \mathrm{ApCD}$ (Figure $6 \mathrm{~B}$ ) and are largely diminished in Synechocystis $\triangle \mathrm{ApcF}$ (Figure $6 \mathrm{C}$ ). The slowest component in the case of Synechocystis $\triangle \mathrm{ApcD}$ and Synechocystis $\triangle \mathrm{ApcF}$ has considerable emission at $650-660 \mathrm{~nm}$, indicating that EET from the corresponding pigments is rather inefficient, possibly due to disconnection of some PBSs as was concluded for Synechocystis $\triangle \mathrm{apcF}$ in ${ }^{[29]}$.

In Figures 6D-F reconstructed steady-state emission spectra of dark-and light-adapted Synechocystis WT, $\triangle \mathrm{ApcD}$ and $\triangle \mathrm{ApcF}$ cells are presented. From the reconstructed steadystate fluorescence spectra, the fluorescence decrease in state ll was found to be $19.2 \pm 1.0 \%$, 
$-3.3 \pm 1.0 \%$ and $10.9 \pm 2.9 \%$ for Synechocystis $\mathrm{WT}, \triangle \mathrm{ApcD}$, and $\triangle \mathrm{ApcF}$, respectively. From a comparison of the time-resolved and reconstructed steady-state emission spectra of the Synechocystis WT and its $\triangle \mathrm{ApcD}$ mutant, it seems that the $\triangle \mathrm{ApcD}$ cells are fixed in state I as was observed before ${ }^{[28]}$. The fluorescence ratio $F_{660} / F_{680}$ in the $\Delta \mathrm{ApcD}$ mutant is higher as compared to the one observed for state I in WT cells, indicating that energy transfer from the PBSs to one or both photosystems is less efficient. In the absence of PSIl some loss of energy transfer to PSI was found at room temperature in Synechocystis $6803 \triangle A p C D$ [28]. However, in ${ }^{[29]}$ it was indicated that the absence of $\triangle A p c D$ does not affect the energy transfer from PBSs to the photosystems in Synechocystis 6803.

The higher $F_{660} / F_{680}$ ratio in the $\triangle \mathrm{ApcF}$ mutant signifies inefficient EET from high-energy pigments in the PBSs to either low-energy pigments in the PBSs or the photosystems. ApcF has been found essential for energy transfer to both photosystems in Synechocystis ${ }^{[28,29]}$. Due to the inefficient EET to the photosystems in Synechocystis $\triangle \mathrm{ApcF}$, the slowest component in figure $6 \mathrm{C}$ might contain a considerable contribution from the PBS terminal emitters. The diminished characteristic fluorescence changes in the dark-and light-adapted Synechocystis $\triangle A$ ApcF cells as compared to WT cells are concluded to be due to less efficient EET from the PBS core to either PSII or to both photosystems.

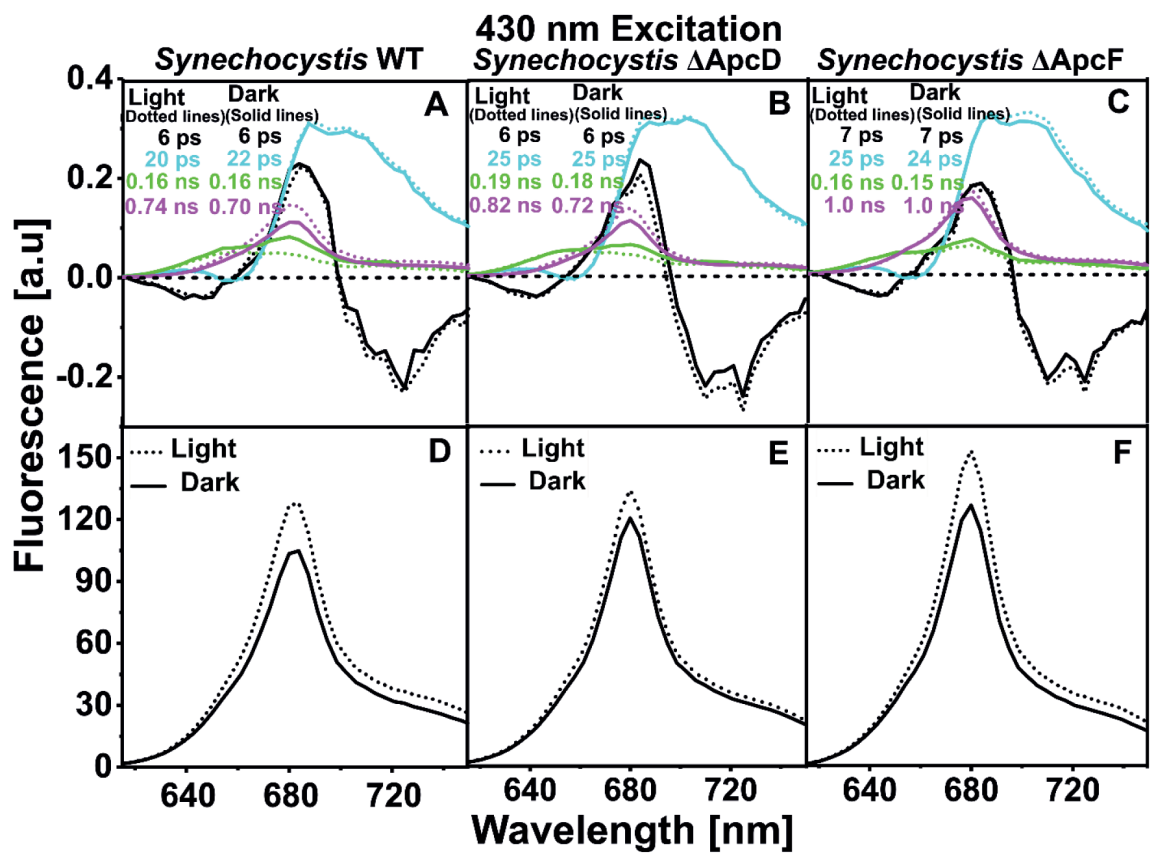

Figure 7. $(A, B, C)$ four-components DAS of Synechocystis WT, Synechocystis $\triangle A p c D$ and Synechocystis $\Delta$ ApcF, respectively in state I (dotted lines) and state II (solid lines). The 20 ps DAS in each case is multiplied by 0.5 to make the two slowest DAS better visible. DAS-associated lifetimes are presented at the top with corresponding colours. In figure $C$ the lifetime of the slowest component was fixed to $1.0 \mathrm{~ns}$. (D,E,F) reconstructed steady-state spectra for Synechococcus WT, Synechococcus $\triangle A p c D$ and Synechococcus $\Delta A p c F$, respectively in state I (dotted lines) and state II (solid lines). 
Upon $430 \mathrm{~nm}$ excitation, the resulting four DAS components for Synechocystis WT, $\triangle A p C D$ and $\triangle \mathrm{ApcF}$ are shown in figure $7 \mathrm{~A}-\mathrm{C}$ and the reconstructed steady-state fluorescence spectra in Figure 7D-F. From the reconstructed steady-state fluorescence spectra, the fluorescence decrease in state II was found to be $20.9 \pm 1.6 \%, 9.4 \pm 1.1 \%$ and $12.7 \pm 3.9 \%$ for Synechocystis WT, $\triangle \mathrm{ApcD}$, and $\triangle \mathrm{ApcF}$, respectively. The fluorescence changes, characteristic for state transitions are observable both for WT and mutant cells but they are far less outspoken than for Synechococcus. However, for mutant cells (especially for $\triangle \mathrm{ApcD}$ ) the differences are smaller than for WT cells.

\section{Discussion}

In this work we applied time-resolved fluorescence spectroscopy to study state transitions in the cyanobacteria Synechococcus sp. PCC 7942, Synechocystis sp. PCC 6803 and their $\triangle \mathrm{ApcD}$ and $\triangle \mathrm{ApcF}$ mutants at room temperature. The most important observation is the decrease in amplitude of the slowest decay component of $\sim 1 \mathrm{~ns}$ with a peak around 680 $\mathrm{nm}$ in state II, and the concomitant increase of the amplitude of the $\sim 0.2$-ns component, upon $\mathrm{Chl} a$ excitation. This implies that there is a significant shortening of the average lifetime of the PSII fluorescence, which corresponds to a decrease of PSII fluorescence in the (reconstructed) steady-state spectra. Such an increase of the $\sim 0.2 \mathrm{~ns}$ component was also observed in an earlier study performed at room temperature but in that case $\mathrm{Chl} a$ excitation was not selective and the increase was ascribed to PBS fluorescence ${ }^{[44]}$. However, when we selectively excite PBSs we observe a similar increase of the $\sim 0.2 \mathrm{~ns}$ component as observed for selective $\mathrm{Chl} a$ excitation. Therefore, in agreement with the $77 \mathrm{~K}$ results reported in ${ }^{[31]}$, we conclude that reversible quenching of PSII is the main mechanism to balance the photochemistry of the two photosystems during state transitions. This quenching mechanism is apparently not affected by a change from room temperature to $77 \mathrm{~K}$.

In the case of PBS excitation, the decrease of PSII fluorescence in state II (Figures 4 and 6) can in principle be due to (i) a decrease of the absorption cross-section/functional antenna size of PSII and/or (ii) quenching of (a fraction of) of the PSII complexes. A decrease of the absorption cross-section/functional antenna size of PSII in state II was ascribed earlier to migration of PBSs from PSII to PSI in the mobile antenna model ${ }^{[15-18]}$. The spillover model ${ }^{[20,50-52]}$ on the other hand, ascribed quenching of PSII in state II to EET to PSI, whereas also a combination of both models was proposed ${ }^{[20,25]}$. All these models predict an increase of EET to PSI in state II at the expense of excitations in PSII. In principle this should lead to an increase of PSI fluorescence in state II. At room temperature such an increase in PSI fluorescence might be difficult to observe due to the relatively slow EET from PBS to PSI ( 0.15-0.17 ns) followed by fast excitation trapping in PSI ( 20 ps). However, also at 77K where the PSI fluorescence kinetics is far slower ( 1.0 ns) no increase could be observed 
for state II, both for wild-type Synechococcus and Synechocystis ${ }^{[7,31]}$. Therefore, Ranjbar Choubeh et al. concluded that neither migration of PBSs from PSII to PSI, nor spillover of excitation energy from PSII to PSI is involved in the transition from state I to state II and fluorescence changes were ascribed to reversible direct quenching of the PSII core ${ }^{[31]}$. In our room-temperature measurements on WT cells upon PBS excitation a fluorescence decrease of $35.8 \pm 2.5 \%$ and $19.2 \pm 1.0 \%$ in state II was observed, for S. elongatus and Synechocystis, respectively. Upon $\mathrm{Chl} a$ excitation, the decrease in PSII fluorescence was $39.6 \pm 1.1 \%$ for S. elongatus and $20.9 \pm 1.6 \%$ for Synechocystis. The similar decrease in the amount of PSII fluorescence in state II, both upon PBS and Chl $a$ excitation, supports the conclusion in ${ }^{[31]}$ that the PSII core is partially quenched. Although such a difference is also observed for dark-and light-adapted Synechocystis $\triangle A p c D$ (Figure $7(B, E)$ ) upon Chl $a$ excitation at $430 \mathrm{~nm}$, albeit to a lesser extent, it is totally absent upon PBS excitation at $577 \mathrm{~nm}$ (Figure 6(B,E)). This observation may cast some doubt on the implicit conclusion in ${ }^{[31]}$ that PBSs play no role in state II. Instead PBSs might show reduced EET to PSII in state II in WT cells, either by disconnecting from PSII or by quenching excitations. This role appears to be disabled with the deletion of ApcD in Synechocystis. The disabled role of PBSs alone, however, does not explain why PBS-absorbed energy is not quenched the PSIl core after PBS excitation as is the case for $\mathrm{Chl} a$ absorbed excitations in this mutant. A solution to this problem was suggested by McConnell et al. ${ }^{[20]}$. They proposed that small scale membrane changes in state II bring PSII and PSI closer together, such that the rate of excitation transfer from PBSs (shared by both photosystems) to PSI increases, whereas also spillover of excitation energy from PSII to PSI occurs. At the same time it was proposed that the PBS-absorbed excitations are transferred directly to the PSII reaction centres and cannot spill over to PSI via CP43 and CP47 antennas, whereas excitations created in CP43 or CP47 upon $\mathrm{Chl} a$ excitation can spill over to PSI.

However, the proposal of McConnell et al. cannot explain our data. The excited-state lifetime of the PSIl core with closed RCs is of the order of $1 \mathrm{~ns}$ and there is plenty of time for excitations to equilibrate over the entire core, irrespective whether they originate from the PBSs or from the core itself. Therefore, if the core would be quenched due to spillover this should also be observed after PBS excitation, followed by transfer to the PSII core. We therefore believe that a more likely explanation is that PSII is directly quenched in state II leading to less PSII fluorescence while in the absence of ApcD EET from the PBS to PSII is less efficient and therefore leads to more PBS fluorescence, thereby compensating for the loss of PSII fluorescence.

\subsection{Possible mechanisms of PSII quenching in state II}

Because the PSII core is a delicate piece of equipment that can easily be damaged in case of overexcitation, oxygenic photosynthetic organisms have developed photoprotective mechanisms that lead to excitation quenching such that excess excitations are safely 
dissipated as heat. A well-known example is the occurrence of non-photochemical quenching in e.g. plants, green algae, cyanobacteria and diatoms, where the organisms can rapidly switch on and off antenna quenching mechanisms that protect PSII from overexcitation ${ }^{[53]}$. In many cases this requires the involvement of carotenoids in the outer antenna system that function as excitation quenchers in NPQ conditions (see e.g. [54-56]). This heat dissipation occurs very rapidly ( $<1 \mathrm{~ns}$ after excitation) and with the use of optoacoustic experiment extra heat dissipation was indeed observed for thylakoid membranes in NPQ conditions within $1.4 \mu$ s after excitation with a laser flash ${ }^{[57]}$.

On the other hand, quenching can also occur in the PSII RC. This has been suggested as an effective protective mechanism against photodamage in several cyanobacteria ${ }^{[58,59]}$ and other organisms ${ }^{[60,61]}$. Also in a number of studies on desiccated lichens it was concluded that (auxiliary) quenching occurs within the PSII reaction center ${ }^{[62-66]}$ and this mechanism was recently also proposed for the desert cyanobacterium Leptolyngbya ohadii [67]. The underlying molecular mechanism of $\mathrm{RC}$ quenching might be due to radiationless charge recombination in the $\mathrm{RC}$, a process that can occur on a time scale of hundreds of microseconds or longer ${ }^{[57]}$. Such a mechanism might also be responsible for the PSII quenching in state II, rather than energy dissipation via one of the carotenoids in the PSII core. Such an explanation might be supported by the fact that independent optoacoustic studies of state transitions in the cyanobacteria Synechococcus $6301^{[68,69]}$ were not able to detect extra heat release in state II within 1.4-2.0 $\mu$ s after excitation. However, we cannot rule out that other mechanisms might be responsible, for instance the formation of quenchers in CP43 or CP47.

\section{Conclusions}

In this work we show that at physiological relevant temperatures the average excited-state lifetime of PSII in state II becomes shorter as compared to state I due to partial quenching of the PSII excitations, balancing the photochemistry of the two photosystems. This was proposed before based on fluorescence measurements at $77 \mathrm{~K}$ but now we obtained for the first time room-temperature picosecond fluorescence data that are fully consistent with that proposal for Synechocystis and Synechococcus, WT, $\triangle A p c D$ and $\triangle A p c F$ strains. One possible explanation for this quenching might be radiationless charge recombination in the RC of PSII in state II but we can certainly not rule out other quenching mechanisms, for instance in CP43 or CP47. Synechocystis $\triangle \mathrm{ApCD}$ spectra in state I and II differ upon ChI $a$ excitation but show hardly any difference upon PBS excitation. We demonstrate that an earlier model ${ }^{[20]}$ is insufficient to explain the phenomenon and we provide an alternative explanation. 


\section{References}

1. Croce, R., et al., Light Harvesting in Photosynthesis. 2018, CRC Press: Boca Raton, FL.

2. Croce, R. and H. van Amerongen, Natural strategies for photosynthetic light harvesting. Nature Chemical Biology, 2014. 10: p. 492.

3. P. Horton, a. A. V. Ruban, and R.G. Walters, REGULATION OF LIGHTHARVESTING IN GREEN PLANTS. Annual Review of Plant Physiology and Plant Molecular Biology, 1996. 47(1): p. 655-684.

4. Clayton, R.K., Biophysical problems of photosyntheis, in Science. 1965. p. 1346-1354.

5. Satoh, K. and D.C. Fork, The relationship between state II to state I transitions and cyclic electron flow around photosystem I. Photosynthesis Research, 1983. 4(1): p. 245-256.

6. Mullineaux, C.W. and J.F. Allen, State 1-State 2 transitions in the cyanobacterium Synechococcus 6301 are controlled by the redox state of electron carriers between Photosystems I and II. Photosynthesis Research, 1990. 23(3): p. 297-311.

7. Chukhutsina, V., et al., Cyanobacterial Light-Harvesting Phycobilisomes Uncouple From Photosystem I During Dark-To-Light Transitions. Scientific reports, 2015. 5: p. 14193-14193.

8. Murata, N., Control of excitation transfer in photosynthesis I. Light-induced change of chlorophyll a fluoresence in Porphyridium cruentum. Biochimica et Biophysica Acta (BBA) - Bioenergetics, 1969. 172(2): p. 242-251.

9. Bonaventura, C. and J. Myers, Fluorescence and oxygen evolution from Chlorella pyrenoidosa. Biochimica et Biophysica Acta (BBA) - Bioenergetics, 1969. 189(3): p. 366-383.

10. Mullineaux, C.W. and D. Emlyn-Jones, State transitions: an example of acclimation to low-light stress. Journal of Experimental Botany, 2004. 56(411): p. 389-393.

11. Mullineaux, C.W., Excitation energy transfer from phycobilisomes to Photosystem $I$ in a cyanobacterium. Biochimica et Biophysica Acta (BBA) - Bioenergetics, 1992. 1100(3): p. 285292.

12. Staehelin, L.A. and C.J. Arntzen, Regulation of chloroplast membrane function: protein phosphorylation changes the spatial organization of membrane components. The Journal of cell biology, 1983. 97(5 Pt 1): p. 1327-1337.

13. Allen, J.F., et al., Chloroplast protein phosphorylation couples plastoquinone redox state to distribution of excitation energy between photosystems. Nature, 1981. 291 (5810): p. 25-29.

14. Wientjes, E., H. van Amerongen, and R. Croce, LHCII is an antenna of both photosystems after long-term acclimation. Biochimica et Biophysica Acta (BBA) - Bioenergetics, 2013. 1827(3): p. 420-426.

15. Allen, J.F., C.E. Sanders, and N.G. Holmes, Correlation of membrane protein phosphorylation with excitation energy distribution in the cyanobacterium Synechococcus 6301. FEBS Letters, 1985. 193(2): p. 271-275.

16. Mullineaux, C.W. and J.F. Allen, Fluorescence induction transients indicate dissociation of Photosystem II from the phycobilisome during the State-2 transition in the cyanobacterium Synechococcus 6301. Biochimica et Biophysica Acta (BBA) - Bioenergetics, 1988. 934(1): p. 96107. 
17. E. Sanders, C. and J. Allen, The $18.5 \mathrm{kDa}$ Phosphoprotein of the Cyanobacterium Synechococcus 6301 : A Component of the Phycobilisome. 1987. p. 761-764.

18. Allen, J.F. and N.G. Holmes, A general model for regulation of photosynthetic unit function by protein phosphorylation. FEBS Letters, 1986. 202(2): p. 175-181.

19. Mullineaux, C.W., M.J. Tobin, and G.R. Jones, Mobility of photosynthetic complexes in thylakoid membranes. Nature, 1997. 390(6658): p. 421-424.

20. McConnell, M.D., et al., Regulation of the distribution of chlorophyll and phycobilin-absorbed excitation energy in cyanobacteria. A structure-based model for the light state transition. Plant physiology, 2002. 130(3): p. 1201-1212.

21. Bruce, D., et al., Mechanism of the light state transition in photosynthesis. IV. Picosecond fluorescence spectroscopy of Anacystis nidulans and Porphyridium cruentum in state 1 and state 2 at 77 K. Biochimica et Biophysica Acta (BBA) - Bioenergetics, 1985. 806(2): p. 237-246.

22. Olive, J., et al., Ultrastructure and light adaptation of phycobilisome mutants of Synechocystis PCC 6803. Biochimica et Biophysica Acta (BBA) - Bioenergetics, 1997. 1319(2): p. 275-282.

23. Olive, J., et al., Randomization of the EF particles in thylakoid membranes of synechocystis 6714 upon transition from state I to state II. FEBS Letters, 1986. 208(2): p. 308-312.

24. Liu, H., et al., Phycobilisomes supply excitations to both photosystems in a megacomplex in cyanobacteria. Science (New York, N.Y.), 2013. 342(6162): p. 1104-1107.

25. Koblizek, M., J. Komenda, and J. Masojídek, State Transitions in the Cyanobacterium Synechococcus PCC 7942. Mobile Antenna or Spillover? 1998. p. 213-216.

26. Dong, C. and J. Zhao, $A p C D$ is required for state transition but not involved in blue-light induced quenching in the cyanobacterium Anabaena sp. PCC7120. Vol. 53. 2008. 3422-3424.

27. Dong, C., et al., ApcD is necessary for efficient energy transfer from phycobilisomes to photosystem I and helps to prevent photoinhibition in the cyanobacterium Synechococcus sp. PCC 7002. Biochimica et Biophysica Acta (BBA) - Bioenergetics, 2009. 1787(9): p. 1122-1128.

28. Ashby, M.K. and C.W.Mullineaux, The role of ApcD and ApcFinenergy transfer from phycobilisomes to PS I and PS II in a cyanobacterium. Photosynthesis Research, 1999. 61(2): p. 169-179.

29. Calzadilla, P.I., et al., Different roles for ApCD and ApcF in Synechococcus elongatus and Synechocystis sp. PCC 6803 phycobilisomes. Biochimica et Biophysica Acta (BBA) - Bioenergetics, 2019.

30. Emlyn-Jones, D., M.K. Ashby, and C.W. Mullineaux, A gene required for the regulation of photosynthetic light harvesting in the cyanobacterium Synechocystis 6803. Molecular Microbiology, 1999. 33(5): p. 1050-1058.

31. Ranjbar Choubeh, R., et al., State transitions in the cyanobacterium Synechococcus elongatus 7942 involve reversible quenching of the photosystem II core. Biochimica et Biophysica Acta (BBA) - Bioenergetics, 2018. 1859(10): p. 1059-1066.

32. Adir, N., Elucidation of the molecularstructures of components of the phycobilisome: reconstructing a giant. Photosynthesis Research, 2005. 85(1): p. 15-32.

33. Glazer, A.N., et al., The structure of a «simple phycobilisome. Annales de I'Institut Pasteur / Microbiologie, 1983. 134(1, Supplement B): p. 159-180. 
34. Arteni, A.A., G. Ajlani, and E.J. Boekema, Structural organisation of phycobilisomes from Synechocystis sp. strain PCC6803 and their interaction with the membrane. Biochimica et Biophysica Acta (BBA) - Bioenergetics, 2009. 1787(4): p. 272-279.

35. Jallet, D., M. Gwizdala, and D. Kirilovsky, $A p c D, A p c F$ and $A p c E$ are not required for the Orange Carotenoid Protein related phycobilisome fluorescence quenching in the cyanobacterium Synechocystis PCC 6803. Biochimica et Biophysica Acta (BBA) - Bioenergetics, 2012. 1817(8): p. 1418-1427.

36. Ajlani, G., et al., Phycobilisome core mutants of Synechocystis PCC 6803. Biochimica et Biophysica Acta (BBA) - Bioenergetics, 1995. 1231(2): p. 189-196.

37. Capuano, V., et al., The "anchor polypeptide" of cyanobacterial phycobilisomes. Molecular characterization of the Synechococcus sp. PCC 6301 apce gene. The Journal of biological chemistry, 1991. 266(11): p. 7239-7247.

38. Van Stokkum, I.H.M., et al., (Sub)-Picosecond Spectral Evolution of Fluorescence Studied with a Synchroscan Streak-Camera System and Target Analysis, in Biophysical Techniques in Photosynthesis, T.J. Aartsma and J. Matysik, Editors. 2008, Springer Netherlands: Dordrecht. p. 223-240.

39. Snellenburg, J., J., et al., Glotaran: A Java-based graphical user interface for the R package TIMP. Journal of Statistical Software, 2012. 49(3): p. -.

40. Van Stokkum, I. and K. M. Mullen, TIMP: An R Package for Modeling Multi-way Spectroscopic Measurements. 2007.

41. Kirilovsky, D., Modulating energy arriving at photochemical reaction centers: Orange carotenoid protein-related photoprotection and state transitions. Photosynthesis research, 2014. 126.

42. Calzadilla, P.I. and D. Kirilovsky, Revisiting cyanobacterial state transitions. Photochemical \& Photobiological Sciences, 2020. 19(5): p. 585-603.

43. Calzadilla, P.I., et al., The Cytochrome $b$ (6) $f$ Complex Is Not Involved in Cyanobacterial State Transitions. The Plant cell, 2019. 31(4): p. 911-931.

44. Mullineaux, C.W., et al., Picosecond time-resolved fluorescenceemission spectra indicate decreased energy transfer from the phycobilisome to Photosystem II in light-state 2 in the cyanobacterium Synechococcus 6301. Biochimica et Biophysica Acta (BBA) - Bioenergetics, 1990. 1015(2): p. 231-242.

45. Tian, L., S. Farooq, and H. Van Amerongen, Probing the picosecond kinetics of the photosystem II core complex in vivo. Physical chemistry chemical physics : PCCP, 2013. 15.

46. van J. J, T., et al., Light Harvesting and State Transitions in Cyanobacteria. Vol. 111. 1998.

47. Acuña, A.M., et al., Resolving the contribution of the uncoupled phycobilisomes to cyanobacterial pulse-amplitude modulated (PAM) fluorometry signals. Photosynthesis research, 2016. 127(1): p. 91-102.

48. Tian, L., et al., Picosecond kinetics of light harvesting and photoprotective quenching in wildtype and mutant phycobilisomes isolated from the cyanobacterium Synechocystis PCC 6803. Biophysical journal, 2012. 102(7): p. 1692-1700. 
49. Tian, L., et al., Site, Rate, and Mechanism of Photoprotective Quenching in Cyanobacteria. Journal of the American Chemical Society, 2011. 133(45): p. 18304-18311.

50. Biggins, J. and D. Bruce, Regulation of excitation energy transfer in organisms containing phycobilins. Photosynthesis Research, 1989. 20(1): p. 1-34.

51. Bruce, D., S. Brimble, and D.A. Bryant, State transitions in a phycobilisome-less mutant of the cyanobacterium Synechococcus sp. PCC 7002. Biochimica et Biophysica Acta (BBA) Bioenergetics, 1989. 974(1): p. 66-73.

52. Salehian, O. and D. Bruce, Distribution of excitation energy in photosynthesis: quantification of fluorescence yields from intact cyanobacteria. Journal of Luminescence, 1992. 51(1): p. 91-98.

53. Ruban, A.V., Nonphotochemical Chlorophyll Fluorescence Quenching: Mechanism and Effectiveness in Protecting Plants from Photodamage. Plant physiology, 2016. 170(4): p. 19031916.

54. Ruban, A.V., et al., Identification of a mechanism of photoprotective energy dissipation in higher plants. Nature, 2007. 450(7169): p. 575-578.

55. Holt, N.E., et al., Carotenoid Cation Formation and the Regulation of Photosynthetic Light Harvesting. Science, 2005. 307(5708): p. 433.

56. Mascoli, V., et al., Capturing the Quenching Mechanism of Light-Harvesting Complexes of Plants by Zooming in on the Ensemble. Chem, 2019. 5(11): p. 2900-2912.

57. Mullineaux, C.W., A.V. Ruban, and P. Horton, Prompt heat release associated with $\triangle \mathrm{pH}$ dependent quenching in spinach thylakoid membranes. Biochimica et Biophysica Acta (BBA) Bioenergetics, 1994. 1185(1): p. 119-123.

58. Sane, P., et al., A Transient Exchange of the Photosystem II Reaction Center Protein D1:1 with D1:2 during Low Temperature Stress ofSynechococcus sp. PCC 7942 in the Light Lowers the Redox Potential of QB. The Journal of biological chemistry, 2002. 277: p. 32739-45.

59. Cser, K. and I. Vass, Radiative and non-radiative charge recombination pathways in Photosystem II studied by thermoluminescence and chlorophyll fluorescence in the cyanobacterium Synechocystis 6803. Biochimica et Biophysica Acta (BBA) - Bioenergetics, 2007. 1767(3): p. 233243.

60. Ivanov, A.G., et al., Low-temperature modulation of the redox properties of the acceptor side of photosystem II: photoprotection through reaction centre quenching of excess energy. Physiologia Plantarum, 2003. 119(3): p. 376-383.

61. Ivanov, A.G., et al., Photosystem II reaction centre quenching: mechanisms and physiological role. Photosynthesis Research, 2008. 98(1): p. 565.

62. Heber, U., Photoprotection of green plants: a mechanism of ultra-fast thermal energy dissipation in desiccated lichens. Planta, 2008. 228(4): p. 641-650.

63. Heber, U., W. Bilger, and V.A. Shuvalov, Thermal energy dissipation in reaction centres and in the antenna of photosystem II protects desiccated poikilohydric mosses against photo-oxidation. Journal of Experimental Botany, 2006. 57(12): p. 2993-3006. 
64. Heber, U., O.L. Lange, and V.A. Shuvalov, Conservation and dissipation of light energy as complementary processes: homoiohydric and poikilohydric autotrophs. Journal of Experimental Botany, 2006. 57(6): p. 1211-1223.

65. Heber, U., M. Azarkovich, and V. Shuvalov, Activation of mechanisms of photoprotection by desiccation and by light: poikilohydric photoautotrophs*. Journal of Experimental Botany, 2007. 58(11): p. 2745-2759.

66. Heber, U., V. Soni, and R.J. Strasser, Photoprotection of reaction centers: thermal dissipation of absorbed light energy vs charge separation in lichensł. Physiologia Plantarum, 2011. 142(1): p. 65-78.

67. Ranjbar Choubeh, R., et al., Photosystem II core quenching in desiccated Leptolyngbya ohadii. Photosynthesis Research, 2020. 143(1): p. 13-18.

68. Mullineaux, C.W., S. Griebenow, and S.E. Braslavsky, Photosynthetic energy storage in cyanobacterial cells adapted to light-states 1 and 2. A laser-induced optoacoustic study. Biochimica et Biophysica Acta (BBA) - Bioenergetics, 1991. 1060(3): p. 315-318.

69. Bruce, D. and O. Salehian, Laser-induced optoacoustic calorimetry of cyanobacteria. The efficiency of primary photosynthetic processes in state 1 and state 2. Biochimica et Biophysica Acta (BBA) Bioenergetics, 1992. 1100(3): p. 242-250. 


\section{Supplementary Information}

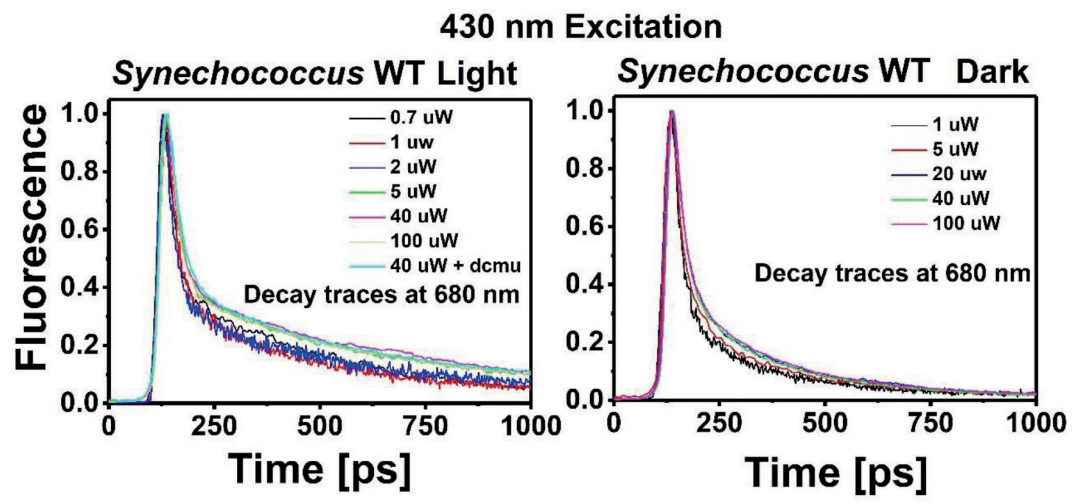

Figure S1. Fluorescence decay traces of Synechococcus cells at $680 \mathrm{~nm}$ obtained with various laser powers after $430 \mathrm{~nm}$ excitation (left) light-adapted cells (state I) and (right) dark-adapted cells (state II). All traces were normalized to each other at their maximum emission. Measurements were performed at room temperature.

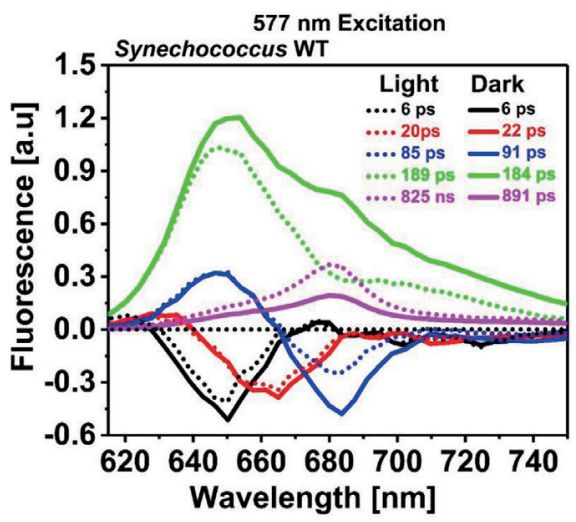

Figure S2. Decay-associated spectra obtained from global fitting of time-resolved fluorescence results upon $577 \mathrm{~nm}$ excitation of Synechococcus WT. Time-resolved fluorescence data was analysed without restraining the slowest lifetime. Cells in state II (solid lines) have a slightly higher value for the slowest component than the cells in state I (dotted lines). 


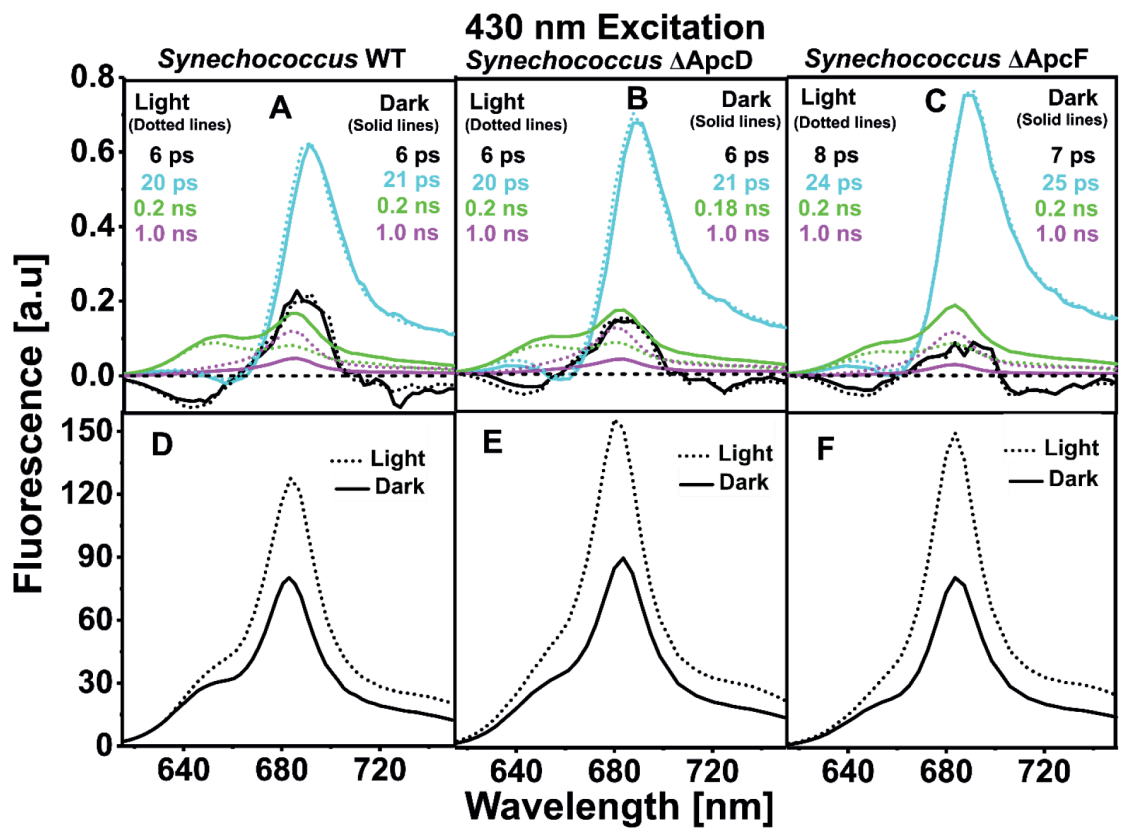

Figure S3. Analysis shown in figure 5 is reproduced here without multiplying the $\sim 20$ ps DAS with 0.5 .

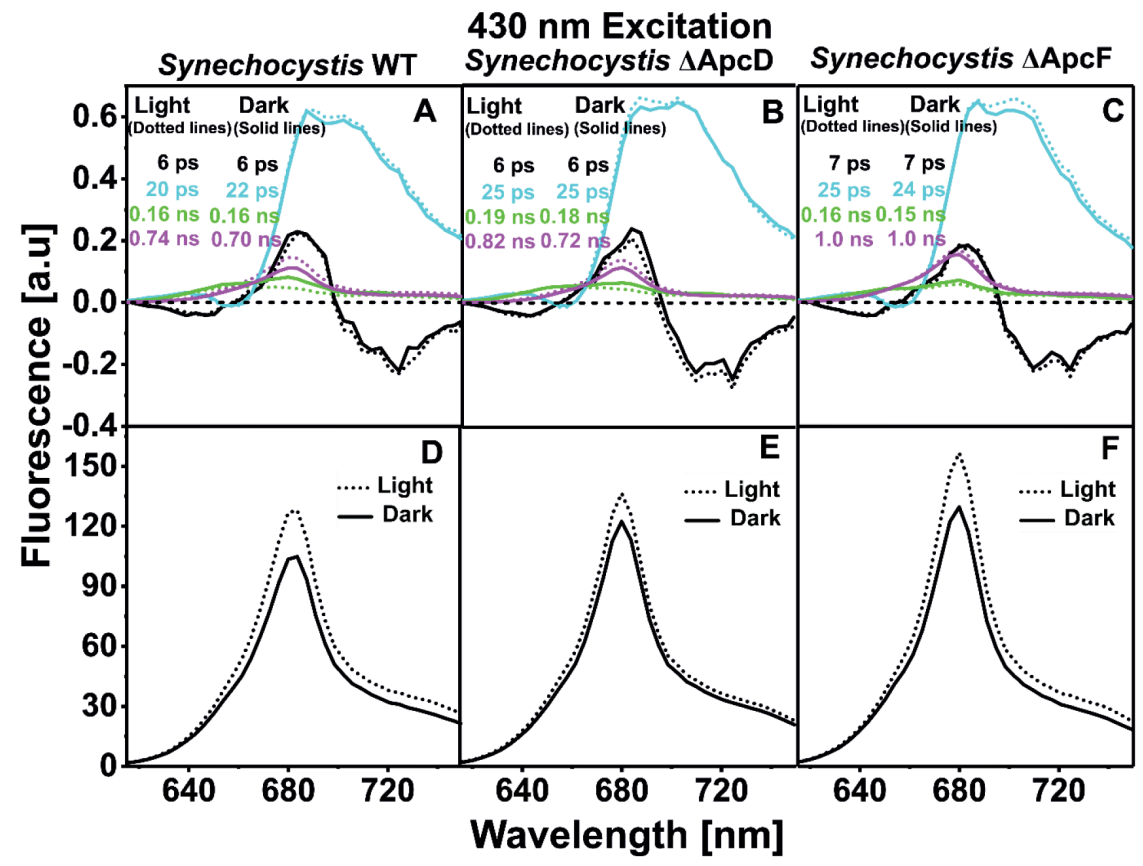

Figure S4. Analysis shown in figure 7 is reproduced here without multiplying the $\sim 20$ ps DAS with 0.5 . 
CHAPTER

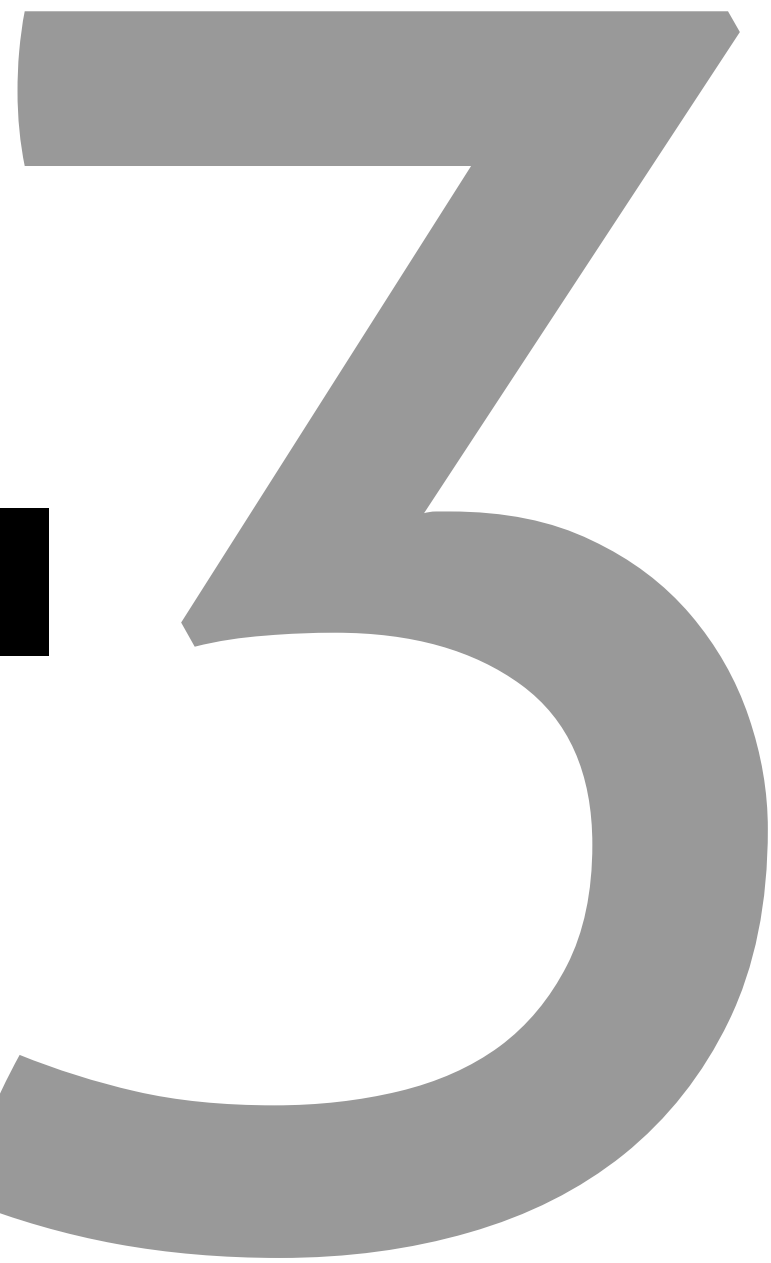




\section{Structural changes in PBS-PSII complexes modulate the excited-state dynamics of PSII during state transitions in cyanobacteria}

Ahmad Farhan Bhatti, Emilie Wientjes, Herbert van Amerongen 
In oxygenic photosynthetic organisms the mechanism of state transitions balances the photochemistry of photosystem I (PSI) and photosystem II (PSII) when one photosystem is overexcited relative to the other. In cyanobacteria there is an increased quenching of PSII complexes in state II. Here, the mechanism of PSIl quenching and the role of phycobilisomes (PBSs) during state transitions is investigated in Synechococcus and Synechocystis. It is shown that a conformational switching of PSIl complexes from an unquenched conformation in state I to the quenched conformation in state II occurs. It is concluded that the interaction of PBSs and PSII complexes is different between Synechococcus and Synechococcus which explains the difference in state transitions between the Synechococcus and Synechocystis $\triangle A p c D$ mutants. It is also concluded that a reversible change in PBS-PSII complexes takes place during state transitions. 


\section{Introduction}

In chapter 2 it was concluded that in cyanobacteria, when the ambient light increases the turnover of PSII photochemistry relative to PSI, the balance between the PSI and PSII photochemistry is restored by the increased quenching of PSII complexes. This conclusion was drawn by comparing the fluorescence kinetics of PSII in state I and state II, both upon direct excitation of the photosystems and the excitation of the photosystems via the phycobilisomes (PBSs). Implicit in this conclusion is that the PBSs are not involved in the light-harvesting regulation mechanism of state transitions in cyanobacteria. In contrast, it is known that in green plants the reshuffling of some LHCIl antennae between the photosystems redistributes the excitation energy (EE) between the photosystems during state transitions ${ }^{[1-4]}$. In green algae, the disconnection of some LHCII complexes from PSII reduces the excitation-energy transfer (EET) to PSII in state II. A large proportion of the disconnected LHCII complexes, aggregates to become quenched, presumably for photoprotection ${ }^{[5]}$.

The mechanism that leads to the quenching of PSII in state II is not known yet for cyanobacteria. At the moment it is difficult to explain the following observations: i) the reduced quenching of PSII in state II in the Synechocystis $\triangle A P C D$ mutant upon direct excitation of PSII together with ii) the apparent absence of PSII quenching in state II upon PBS excitation (chapter 2). This Synechocystis $\triangle A p C D$ mutant is missing one subunit in the PBS core and the fact that this leads to reduced PSIl quenching even upon direct excitation of PSII clearly shows that, at least there must be some involvement of PBSs in the state-transitions mechanism. iii) In contrast in the Synechococcus $\triangle A p c D$ mutant, during state transitions pronounced changes in the fluorescence, similar to the ones in Synechococcus WT are observed, both upon PBS and photosystem excitation. These three experimental observations will be further addressed in the present chapter, trying to answer the question whether PSII quenching is sufficient to explain the PSII fluorescence decrease in state II or whether more is needed. iv) Finally, a strong correlation between state transitions and PBS diffusion was reported for Synechococcus ${ }^{[6]}$, thereby challenging the conclusions that state transitions should entirely be explained by PSIl quenching. On the flip side, these observations may help to provide further insight into the origin of theexcited state quenching of PSII and the mechanism of state transitions.

\section{A hypothesis for the state-transitions mechanism}

\section{The nature of the PSII fluorescence decrease}

From the time-resolved fluorescence data shown and discussed in chapter 2, it is clear that the nature of the PSII fluorescence decrease in state II is due to the quenching of PSII complexes rather than the decrease of the PSII antenna size. 


\section{The origin of PSII quenching}

\section{PBS Excitation (577 $\mathrm{nm})$}
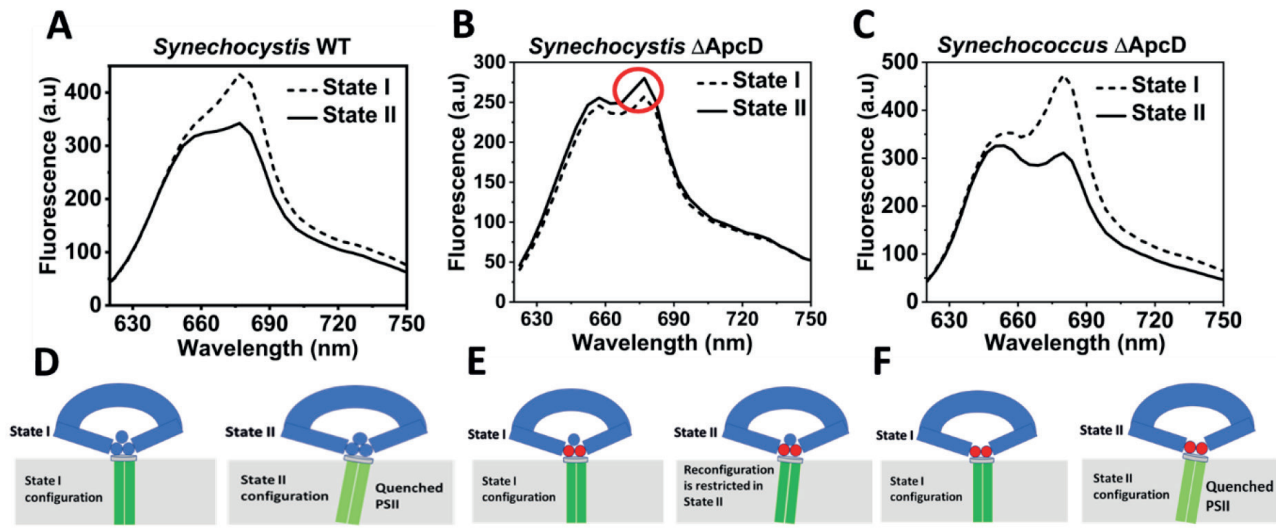

Fig. 1. (A, B, C) Upon PBS excitation $(577 \mathrm{~nm})$, steady-state fluorescence spectra reconstructed from the analysis of time-resolved fluorescence data (see chapter 2, Fig. 4 and Materials and Methods below for explanation) of cells in state I (broken line) and state II (solid line) for (A) Synechocystis WT, (B) Synechocystis $\triangle A p C D$ and (C) Synechococcus $\triangle A p c D$. Measurements were performed at RT. Proposed PBS-PSIl configurations for cells in state I and state II for (D) Synechocystis WT, (E) Synechocystis $\triangle$ ApcD and (C) Synechococcus $\triangle$ ApcD. PBSs with red basal cylinders represent $\triangle A P C D$ mutant PBSs. PSIl dimers with dark green and light green colors, represent unquenched and quenched PSII, respectively. Note that the cartoon diagrams in D, E and F are mere conceptual representations and the sizes of PBSs and PSII complexes are not according to their real scale.

Electron microscopy data shows ${ }^{[7-9]}$ that in cyanobacteria the PBSs are aligned on top of the PSII particles. A PBS that is energetically and physically coupled to PSII(s) can be imagined as a one photosynthetic unit (PBS-PSII) as represented in Fig. 1(D,E,F). In principle, two different propositions can be made to explain the quenching of PSII observed in state II in Synechocystis WT (Fig. 1A). First, PSII complexes become quenched without involving any physical change/reorientation between PBS and PSII. In this case, PBSs have no possibility to influence the quenching process in state II and a comparable quenching of PSII should be observed both in the Synechocystis WT and Synechocystis $\triangle \mathrm{ApcD}$ cells. However, what is shown in Fig. 1B and Fig. S1 is the opposite. The pronounced PSIl quenching, which is observed in Synechocystis WT (Fig. 1A) is inhibited with the deletion of the ApcD protein subunit from the trimer of the basal cylinder of the PBS core (Fig. 1B). This observation leads to the second proposition that a structural (conformational) change in PSII complexes upon transitioning from state I to state II, involving a reorganization of PBS-PSII unit (Fig. 1D) is at the basis of the excited-state quenching observed in Synechocystis WT (Fig.1 A). Using this proposition, the results of Synechocystis $\triangle \mathrm{ApCD}$ (Fig. 1B) and Synechococcus $\triangle A p c D$ (Fig. 1C) can be explained as follows: The deletion of the ApcD unit in Synechocystis alters the binding/interaction of the PBS core with the PSII complexes in such a way that, the reorganization of the PBS-PSII unit and thereby the quenched conformation of PSII in state II is inhibited. Consequently, the majority of PSII complexes remain structurally locked 
in state I as observed upon PBS excitation in Fig. 1B. The inhibition of the reorganization of the PBS-PSII unit in state II in Synechocystis $\triangle \mathrm{ApcD}$ leads to some disconnection of PBSs from PSIl complexes as observed by the slightly higher fluorescence at $\sim 680 \mathrm{~nm}$ from state II cells as compared to state I cells (Fig. 1B). However, in a small fraction of the PBSPSII units of Synechocystis $\triangle A p c D$, reorganization and consequently the quenching of PSII is successful, as $~ 10 \%$ fluorescence decrease from PSII in state II is observed upon $\mathrm{Chl} a$ excitation (Fig. S1). Upon PBS excitation (Fig. 1B), the higher fluorescence from disconnected PBSs masks the small fluorescence decrease due to the quenching of some PSIl complexes in Synechocystis $\triangle \mathrm{ApcD}$.

Contrary to Synechocystis, the deletion of the ApcD unit from Synechococcus does not inhibit PSII quenching in state II (Fig. 1C), showing that the structural/conformational change of the PSII complexes upon transitioning to state II is not inhibited in Synechococcus $\triangle A p c D$. The different effects that the deletion of ApcD unit has in both species, suggest that the PBS cores of Synechococcus and Synechocystis couple/interact slightly differently with PSII in both species. In other words, the PBS-PSII unit in Synechococcus is (possibly in a subtle way) different from the one in Synechocystis.

To summarize the hypothesis, i) EET from PBSs to PSII is similar in both states and only one process, i.e., the increased excited-state quenching of PSII in state II is sufficient to explain the fluorescence decrease from PSII for both, PBS-absorbed and, Chl-a-absorbed $E E$. Therefore, two different EE redistribution processes during state transitions, one for the redistribution of PBS absorbed EE and the other for the redistribution of the Chl $a$ absorbed EE are not necessary to explain the fluorescence decrease. ii) A reversible physical change in the PSII complexes involving an organizational/structural change in PBS-PSII unit is responsible for PSII quenching in state II. iii) The deletion of the ApcD unit stabilizes the PBS-PSII complex and structurally locks the majority of PSII complexes in the state-I configuration in Synechocystis but not in Synechococcus.

\section{Experimental design}

The first important thing is to determine experimentally the number of processes involved in the redistribution of EE during state transitions. In the hypothesis section it is proposed that all the fluorescence changes at PSIl emission during state transitions both in WT and mutant cells can be explained by the presence or inhibition of reversible quenching of PSII. However, two processes for the EE redistribution during state transitions have been proposed in the literature. One process is dependent on the mobility/involvement of PBSs and is responsible for the redistribution of the PBS-absorbed $E E^{[3,6,10-16]}$ and the second process which depends on the diffusion/reorganization of the one or both photosystems in the thylakoid membrane and is considered to be responsible for the redistribution of 
the $\mathrm{EE}$ absorbed by $\mathrm{Chl} a^{[17-24]}$. An experiment is designed to probe the potential presence of the two processes and disentangle them (if both are present) based upon their different kinetics.

PBSs, externally attached to the thylakoid membrane and the photosystems embedded in the densely packed lipid-bilayer, experience very different environments. PBSs are watersoluble proteins and their diffusion along the thylakoid membrane is reported to be much more rapid as compared to the diffusion of the photosystems in the membrane ${ }^{[6]}$. Assuming that there are two processes for the redistribution of EE during state transitions, then in principle the EE regulation process which might depend on the diffusion of the PBSs ${ }^{[6,13,14]}$ might have different kinetics as compared to the one dependent on the diffusion/ reorganization of the photosystems ${ }^{[9,17,20,23]}$. However, in the pure WT cells, the proposed two processes might not be kinetically separable because the change in fluorescence kinetics will be limited by the rate of oxidation/reduction of the plastoquinone pool. Therefore, to separate the two processes, it is necessary to make the kinetics of at least one of the two processes (PBS dependent or membrane dependent) significantly slower than the redox kinetics of plastoquinone pool. The kinetics of the PBS-dependent process can then be probed with the selective excitation of PBSs and compared with the kinetics of a membrane-dependent process using selective $\mathrm{Chl} a$ excitation. It was already shown that the diffusion of the PBSs can be slowed down and completely stopped with high osmotic strength buffers, like $\mathrm{KH}_{2} \mathrm{PO}_{4}{ }^{[6]}$. A strong correlation was found between PBS diffusion and the state-transitions-associated fluorescence changes probed upon PBS excitation. The same amount (0.4-0.5 M) of $\mathrm{KH}_{2} \mathrm{PO}_{4}$ was found to be required to fully inhibit both PBS mobility and state transitions in Synechococcus [6]. Therefore, we have used $\mathrm{KH}_{2} \mathrm{PO}_{4}$ in this work to control the PBS mobility.

The kinetics of state transitions are often monitored by using the pulse-amplitudefluorimetry (PAM) method. With PAM (PAM 101-103) the Chl $a$ fluorescence kinetics can typically be measured using probe light centered at $650 \mathrm{~nm}{ }^{[25]}$. However, in the case of cyanobacteria, $650 \mathrm{~nm}$ is not very selective for the Chls and significant fluorescence is observed also from the PBSs. Furthermore, with the PAM method the fluorescence is collected above $700 \mathrm{~nm}$, which makes it very difficult to disentangle the relative contributions of PBSs and PSII ${ }^{[25]}$ and thus also potential PBS-dependent and membranedependent state transitions processes. In this work, those technical limitation are circumvented by measuring ultrafast fluorescence with a streak camera using excitation light, that selectively excites the PBSs and $\mathrm{Chl} a$. 


\section{Materials and Methods}

\section{Strains and Growth Conditions}

Synechococcus WT, Synechocystis WT and Synechocystis $\triangle$ ApcD strains of cyanobacteria grown in BG-11medium ( $20 \mathrm{ml} / \mathrm{l})$ buffered with sodium bicarbonate $(0.85 \mathrm{~g} / \mathrm{litre})$, and sodium nitrate ( $1.75 \mathrm{~g} /$ litre) at $\mathrm{pH} \sim 8.0$ were used in this work. Medium for Synechocystis $\triangle \mathrm{ApcD}$ was complemented with chloramphenicol $(20 \mu \mathrm{g} / \mathrm{ml})$. All strains were grown at $30^{\circ} \mathrm{C}$ under white light illumination at $50 \mu \mathrm{mol}$ photons $\mathrm{m}^{-2} \mathrm{~s}^{-1}$ in $250 \mathrm{ml}$ flasks shaken at $100 \mathrm{rpm}$, containing culture volumes of $60 \mathrm{ml}$.

\section{Sample Preparation}

All the WT and mutant cells were harvested during their logarithmic growth phase at optical density $\sim 0.6-0.7$ at $800 \mathrm{~nm}\left(O D_{800}\right.$ for a path length of $\left.1 \mathrm{~cm}\right)$. Cells were diluted with their respective fresh medium to $O D_{630} \sim 0.35$ as measured with a Cary 4000 UVVis spectrophotometer with integrating sphere. Potassium-phosphate $\left(\mathrm{KH}_{2} \mathrm{PO}_{4}\right)$ buffer (1 M) was prepared in growth media. To drive the cells to state I and II, they were kept in blue light (of $452 \pm 40 \mathrm{~nm}$ at $\sim 40 \mu \mathrm{mol}$ photons $\mathrm{m}^{-2} \mathrm{~s}^{-1}$ intensity) and darkness, respectively, for about 30 minutes. Measurements were performed on both pure cells and potassiumphosphate-incubated (0.1-0.4 M) cells from the same biological sample. For both pure cells and potassium-phosphate-incubated cells, the cell density of the sample was kept approximately the same. To prepare $\mathrm{KH}_{2} \mathrm{PO}_{4}$-incubated (0.1-0.4 M) cells, $10 \mathrm{ml}$ volume of pure cells was centrifuged (at 1,500 $\mathrm{g}$ for 10 minutes), and 1-4 $\mathrm{ml}$ supernatant was replaced with the same volume of $1 \mathrm{M} \mathrm{KH}_{2} \mathrm{PO}_{4}$. The $\mathrm{KH}_{2} \mathrm{PO} 4$-incubation time prior to the measurements was $\sim 30$ minutes for Synechococcus and 60-75 minutes for Synechocystis WT and Synechocystis $\triangle \mathrm{ApcD}$. The $\mathrm{pH}$ in all cases was adjusted to $\sim 7.5$ prior to adapting or fixing the cells in state I or state II.

\section{Streak-Camera Measurements}

To perform picosecond fluorescence measurements, cells were injected between the two glass plates of a home-built circular cuvette of $7 \mathrm{~cm}$ diameter ${ }^{[26]}$. The cuvette was simultaneously rotated at 40 r.p.m. and translated over a distance of $4 \mathrm{~cm}$ at 20 times per minute. Streak-camera measurements upon PBS excitation $(577 \mathrm{~nm})$ and $\mathrm{Chl} a$ excitation $(430 \mathrm{~nm})$ and the analysis of resulting time-resolved fluorescence were performed in a similar way as described in the Materials and Methods section of chapter 2. All measurements were performed with closed PSII reaction-centres at room temperature as described in chapter 2. For all our measurements the time-resolved fluorescence is described with five components after $577 \mathrm{~nm}$ excitation. For each measurement the obtained DAS were summed, which provided the estimated fluorescence spectrum at $t=0$, i.e. immediately upon excitation and this $t=0$ spectrum was normalized to a value of 1 in the maximum. All the individual DAS were corrected with the same scaling factor. 
Steady-state fluorescence spectra were subsequently calculated as a sum of the products of amplitudes (DAS) and their respective decay times and below they are referred to as reconstructed steady-state fluorescence spectra.

\section{Kinetics of state I to state II transition}

Kinetics of state I to state II transition were probed both with PBS and Chl $a$ excitation in pure WT Synechococcus cells (no addition of salt buffer). These cells were first fully adapted to state I in the presence of blue light and the fluorescence decay was recorded. The blue light was then switched off to drive the cells to state II and the PSII fluorescence kinetics were measured during $60 \mathrm{~s}$ after 10, 20, 30 and 40 minutes (Fig. 2A and 2B). The experiments were repeated after incubation with different concentrations of $\mathrm{KH}_{2} \mathrm{PO}_{4}(0.1$, 0.2 and $0.3 \mathrm{M}$ ), but only the data of $0.2 \mathrm{M} \mathrm{KH}_{2} \mathrm{PO}_{4}$ is shown here (Figures $2 \mathrm{C}$ and $2 \mathrm{D}$ ). At 0.1 $\mathrm{M} \mathrm{KH}_{2} \mathrm{PO}_{4}$ there was a large variation in the results of different biological replicates. At 0.3 $\mathrm{M} \mathrm{KH}_{2} \mathrm{PO}_{4}$ state transitions were almost fully inhibited. The cells were fully adapted to state I prior to the addition of salt. The salt incubation time was $\sim 30$ minutes.

Probing the kinetics of the state I to state II transition both, upon PBS excitation (Fig. 2A) and $\mathrm{Chl} a$ excitation (Fig. 2B) shows that in pure Synechococcus WT cells the transition to state II is nearly complete within 10 minutes of dark adaptation and after 20 minutes the cells are fully equilibrated in state II. However, in the potassium-phosphate-incubated Synechococcus WT cells (Fig. 2C and 2D) the transition to state II is rather small after 10 minutes of incubation in the dark (see red line in Fig. 2C and 2D) and the transition to state II continues up to 30-40 minutes in the dark (see blue and magenta lines in Fig. 2C and 2D). Furthermore, in $0.2 \mathrm{M} \mathrm{KH}_{2} \mathrm{PO}_{4}$-incubated cells, the amount of PSIl quenching is significantly reduced (Fig. 2C and 2D) as compared to the pure cells (Fig. 2A and Fig. 2B). In a previous study it has been proposed that in $\mathrm{KH}_{2} \mathrm{PO}_{4}$-incubated Synechococcus cells, $\mathrm{KH}_{2} \mathrm{PO}_{4}$ replaces water molecules from the vicinity of PBSs thus reducing the mobility of the water soluble PBSs ${ }^{[6]}$. It is difficult to assume that the incubation of Synechococcus with $\mathrm{KH}_{2} \mathrm{PO}_{4}$ would inhibit the diffusion (if there is) or reorganization of photosystems which are embedded in the membrane lipid bilayer. Therefore, the slowdown of state I to state II kinetics and the reduced magnitude of overall PSII quenching in Fig. $2 \mathrm{C}$ and 2D can be ascribed to the inhibited PBS mobility and stronger/stable coupling of PBSs and photosystems. 


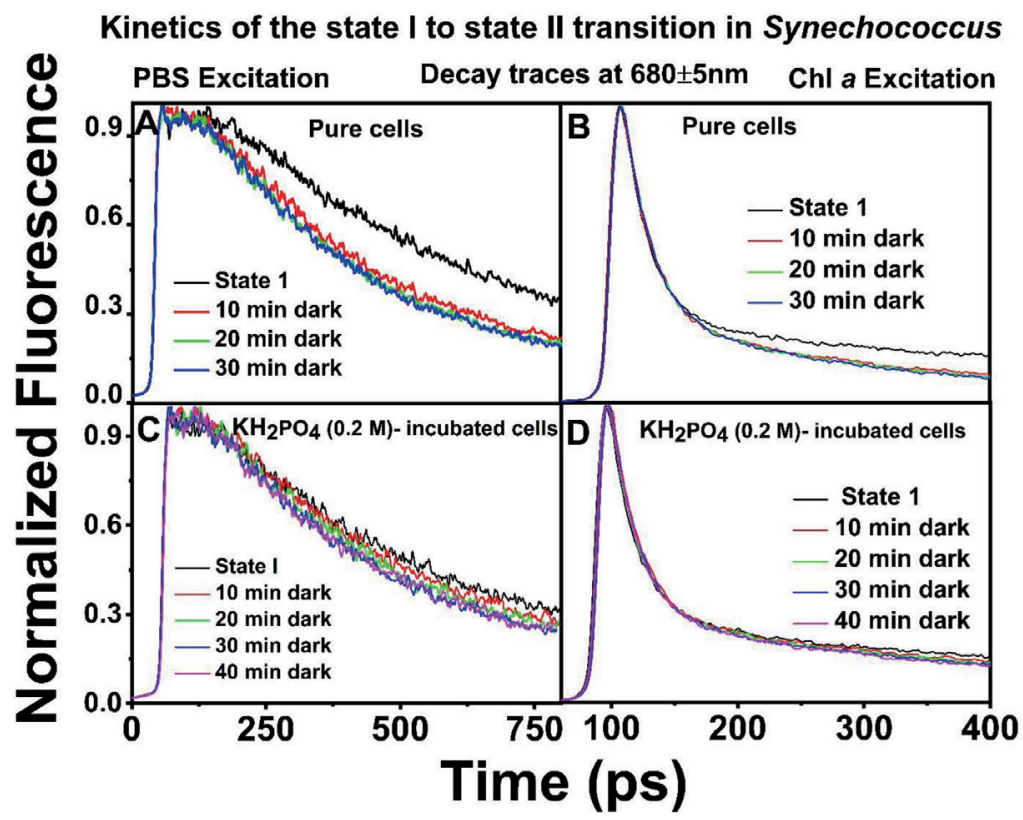

Fig. 2. Upon PBS excitation $(A, C)$ and $C h l$ a excitation $(B, D)$, fluorescence decay traces at $\sim 680 \pm 5 \mathrm{~nm}$ with closed PSII reaction centers of (A, B) pure Synechococcus cells and (C, D) $0.2 \mathrm{M} \mathrm{KH}_{2} \mathrm{PO}_{4}$-incubated cells. Black lines correspond to cells in state I. Red, green, blue and magenta correspond to cells after 10,20, 30 and 40, minutes of dark adaptation, respectively.

The conclusion here that the inhibition of the PSII quenching in state II in potassiumphosphate-incubated cells is due to the stable PBS-PSII units, in principle reconciles with the proposition in the hypothesis section that, in Synechocystis $\triangle A p c D$ stable binding of PBS-PSII units inhibits PSII quenching.

\section{Potassium phosphate as a probe to investigate ultrastructural details}

The initial purpose of using potassium phosphate was to control the PBS diffusion and then measure the kinetics of the state I to state II transition, leading to the results presented in the previous section. However, already at low concentrations of potassium phosphate $(0.1-0.2 \mathrm{M})$ it became noticeable that the salt induces some fluorescence quenching both in Synechococcus and Synechocystis (Fig. S2). Surprisingly, the same concentration of salt had different effects on the reconstructed steady-state fluorescence spectra of Synechococcus and Synechocystis (Fig. S2). Steady-state fluorescence spectra, in principle, contain information about the structural/physical state of the system. Therefore, it is reasonable to assume that the different effects of the potassium phosphate on the steadystate fluorescence profiles of Synechococcus and Synechocystis (Fig. S2) might reflect some structural/physical differences between the two species. Potassium-phosphate-induced quenching is concluded to be structure/physical state sensitive and is used in this work to detect the proposed (see hypothesis section) i) ultrastructural differences between the 
Synechococcus and Synechocystis regarding PBS and photosystems interaction/coupling and ii) physical/structural reorganization of the PBS-PSII unit during state transitions.

Fluorescence upon PBS Excitation

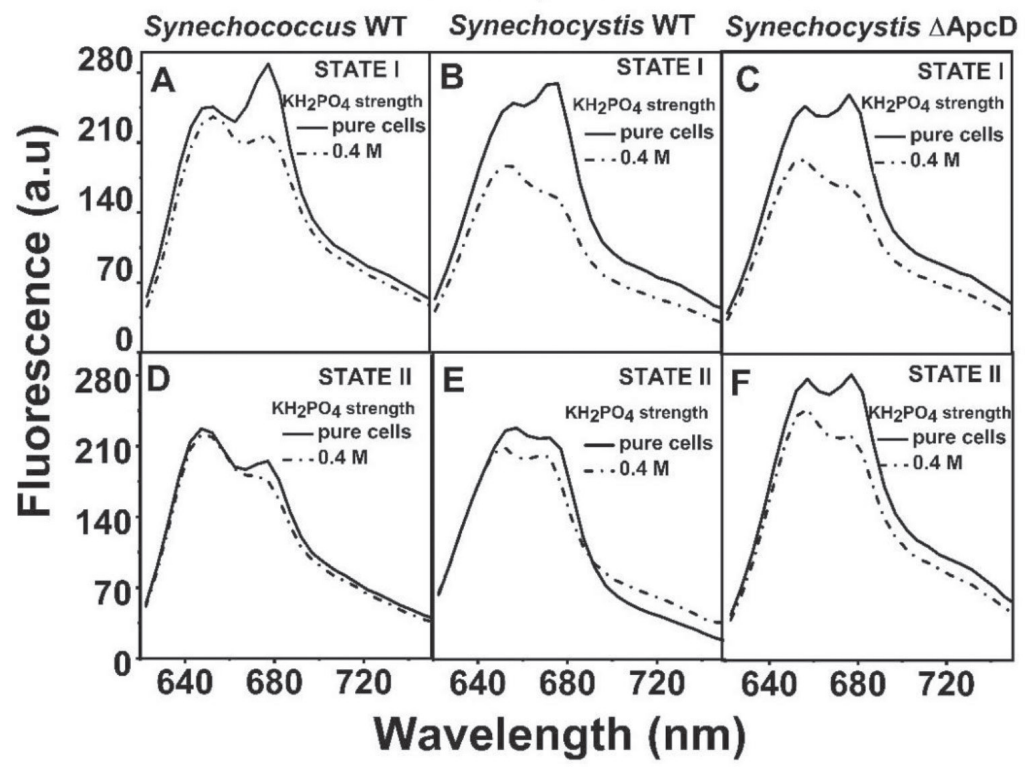

Fig. 3. Steady-state fluorescence spectra reconstructed from the time-resolved fluorescence data (see Materials and Methods for explanation) from cells in state I, i.e. illuminated with blue light (A) Synechococcus WT (B) Synechocystis WT and (C) Synechocystis $\triangle$ ApcD and state II, i.e. kept in the dark (D) Synechococcus WT (E) Synechocystis WT and (F) Synechocystis $\triangle A p C D$. In each case the solid line represents the fluorescence of pure cells and the broken line represents the fluorescence of potassium-phosphate-incubated cells. Measurements on $\mathrm{KH}_{2} \mathrm{PO}_{4}$-incubated Synechococcus cells were performed after 30 minutes of incubation and the incubation time for Synechocystis in each case was at least 60 minutes (see Fig. S4). All measurements were performed at room temperature upon PBS excitation.

Incubation with potassium phosphate induces quenching of PSII in state-I cells of Synechococcus (Fig. 3A) and Synechocystis (Fig. 3B) leading to a fluorescence decrease around $680 \mathrm{~nm}$ (at $\mathrm{APC}_{680}$ and PSII emission). The salt-induced PSII quenching was also observed upon direct excitation of the photosystems (data not shown). However, two significant differences are observed for Synechococcus and Synechocystis: i) in Synechocystis cells, salt-induced PSIl quenching is much stronger than in Synechococcus and ii) in Synechocystis cells there is significant quenching of the PBSs ( $\sim \mathrm{APC}_{655}$ emission) which is not observed for Synechococcus. It is concluded that the salt-induced quenching is not a result of some chemical reaction of salt in cells and the higher salt-induced quenching in Synechocystis as compared to Synechococcus is not related to factors such as variations in membrane-surface charges, or differences in the photosynthetic activity between the two species. These conclusions were reached in supplemetary information. It would be particularly difficult to explain the strong quenching of $\mathrm{PBSs}$ in $\mathrm{KH}_{2} \mathrm{PO}_{4}$-incubated Synechocystis cells and the absence of it in $\mathrm{KH}_{2} \mathrm{PO}_{4}$-incubated Synechococcus cells in one 
of these ways. Instead, it is concluded that the origin of salt-induced quenching (in Fig. $3 \mathrm{~A}-\mathrm{F}$ ) is the modified interaction of PBSs and photosystems in salt-incubated cells as compared to pure cells. The higher salt-induced quenching in Synechocystis as compared to Synechococcus is concluded to show the differences in the interaction details of PBSs with photosystem II in both species. In other words the configuration of PBS-PSII unit in Synechocystis is different than in Synechococcus.

State transitions are characterized by a higher PSII fluorescence intensity in state I as compared to state II. However, at $0.4 \mathrm{M}$ potassium phosphate concentration the PSII quenching in state I cells of Synechocystis is so pronounced that the fluorescence from state I cells (Fig. 3B) is significantly lower than from state II cells (Fig. 3E). This comparison shows that the $\mathrm{KH}_{2} \mathrm{PO}_{4}$-induced quenching of PSII is not related to state II quenching. In the supplementary information it is concluded that (see Fig. S4 and related description) the potassium-phosphate-induced quenching is independent of the dark-light state and the redox state of the plastoquinone pool. Consequently, the much stronger $\mathrm{KH}_{2} \mathrm{PO}_{4}-$ induced quenching of PSII and PBSs in state-I Synechocystis cells (Fig. 3B) as compared to state II cells (Fig. 3E) is concluded to reflect the structural/organizational/physical differences of the PBS-PSII unit in both states. In Synechocystis, the PBS-PSII unit/structure is much more susceptible to the potassium-phosphate-induced quenching in state I than in state II. This conclusion reconciles with the proposition made in the hypothesis section that a structural/conformational change in PSII in state II involves reconfiguration/ reorganization of the PBS-PSII unit. Potassium-phosphate-incubated Synechocystis $\triangle \mathrm{ApcD}$ appears to be the best test of the proposition that the deletion of ApcD subunit locks a population of the PBS-PSII units in the physical configuration of state I while cells are driven to state II conditions and the conclusion that PBS-PSII units in the state-I configuration are more susceptible to potassium-phosphate-induced quenching as compared to the state-II configuration. According to the above-mentioned proposition and conclusion, a higher-potassium-phosphate-induced quenching should be observed in state II cells of Synechocystis $\triangle \mathrm{ApcD}$ as compared to state II cells of Synechocystis WT. Therefore, state transitions in potassium-phosphate-induced Synechocystis $\triangle \mathrm{ApCD}$ were characterized and the results are presented in Fig. $3 \mathrm{C}$ for the cells in state I and in Fig. $3 \mathrm{~F}$ for the cells in state II. In state I a pronounced quenching of PBSs and PSII observed in potassium-phosphateinduced Synechocystis $\triangle A p c D$ appears to be characteristic for this species. However, in state I the quenching in the potassium-phosphate-induced Synechocystis $\triangle A p c D$ (Fig 3C) is somewhat smaller than in the potassium-phosphate-induced Synechocystis WT (Fig. 3B). This difference in quenching can be explained with regard to the proposed differences between the PBS-PSII complexes of Synechocystis WT and Synechocystis $\triangle A p c D$ as a result of the $A p c D$ deletion in the mutant. In chapter 2 a partial disconnection of PBSs and PSII is concluded in Synechocystis $\triangle \mathrm{ApcD}$ as compared to Synechocystis WT. In state II, the potassium-phosphate-induced quenching in Synechocystis $\triangle A p c D$ is less 
pronounced than in state-I (Fig. $3 \mathrm{~B}$ and $3 \mathrm{C}$ ) cells but more pronounced than in state-II cells of Synechocystis WT (Fig. 3E). Higher-salt-induced quenching in Synechocystis $\triangle A p c D$ as compared to Synechocystis WT in state II, is consistent with the proposition that in the Synechocystis $\triangle \mathrm{ApCD}$ cells some PBS-PSII units are physically locked in state I. The results of Synechocystis $\triangle \mathrm{ApcD}$ further confirm the conclusion that the PBS-PSII apparatus locked in the state-I configuration, is more susceptible to the potassium-phosphate-induced quenching than in the state-II configuration. Synechocystis $\triangle A p c D$ results also confirm that potassium-phosphate-induced quenching observed in Fig. 3 is independent of the dark/ light state or the redox state of the plastoquinone pool and indeed reflects the structural/ physical state of the PBSs and PSII.

The main conclusions from this section are summarized as follows. i) The origin of saltinduced quenching in Synechococcus and Synechocystis (Fig. 3) is the modified interaction of PBSs and PSII complexes. ii) The PBS-PSII structure in Synechocystis is more susceptible to the salt-induced quenching than in Synechococcus. iii) The PBS-PSII structure is much more susceptible to salt-induced quenching in state I than in state II, thus revealing the reversible structural changes in PBS-PSII complexes during state transitions in cyanobacteria.

\section{Discussion}

The aim of this work was to elucidate the mechanism of state transitions in cyanobacteria. As a starting point of the investigation, steady-state fluorescence spectra of Synechocystis WT, Synechocystis $\triangle A p c D$ and Synechococcus $\triangle A p c D$ were compared both, in state I and state II, upon PBS excitation (Fig. 1A). From the comparisons made from Fig. 1, a hypothesis was proposed about the nature, origin and mechanism of PSII fluorescence decrease in state II, entailing the following propositions: i) quenching of the PSII complexes in state II explains the PSII fluorescence decrease in Synechocystis WT (Fig. 1A) and Synechococcus $\triangle \mathrm{ApcD}$ (Fig. $1 \mathrm{C}$ ), ii) a structural change in PSIl complexes involving a reconfiguration of the PBS-PSII complex is responsible for the PSII excited-state quenching in state II, iii) the deletion of the ApcD unit from the PBS core, structurally locks the majority of the PSII complexes in the state-I configuration (conformation) in Synechocystis but not in Synechococcus, possibly pointing out some subtle difference between the two species regarding PBS and PSII interaction.

\section{Structural flexibility induces reversible quenching in PSII during state transitions}

As first discussed for Synechocystis WT case in the hypothesis section, in principle, two possibilities can be considered to explain the PSII quenching in state II in Synechococcus WT (Fig. 2A and, 2B). First, PSIl quenching occurs without any structural/conformational change in the PSII complexes. In this case, PBSs have no possibility to influence the PSII 
quenching in state II. However, Fig. 2( $C$ and $D)$ show that the inhibition of the PBS mobility in $0.2 \mathrm{M} \mathrm{KH}_{2} \mathrm{PO}_{4}$-incubated Synechococcus cells significantly reduces the extent of PSII quenching in addition to slowing down the state I to state II kinetics. The kinetics of the state I to state II transition and the extent of PSII quenching in salt-incubated cells (Fig. $2 \mathrm{C}$ and 2D) correlate with the kinetics of PBS diffusion and the amount of uncoupled PBSs. This experiment leads to the conclusion that PSII quenching in state II (Fig. 2A and 2B) is due to a conformational change in the PSII complexes whereas, the interaction between PSIl complexes and PBSs is transient. Incubation of state-1 adapted Synechococcus cells with $0.2 \mathrm{M} \mathrm{KH}_{2} \mathrm{PO}_{4}$ transforms the nature of the interaction between the PBSs and PSII complexes, from transient in pure cells to relatively stable in salt-incubated cells. At all times there is a dynamic equilibrium between the distributions of stable and unstable PBS-PSII complexes. The stable PBS-PSII complexes are concluded to become structurally locked in the state-I configuration, leaving only a small population of unstable/transient PBS-PSIl complexes to take part in the state transitions (Fig. 2C and Fig. 2D). This conclusion is consistent with the analysis of the time-resolved fluorescence data (chapter 2) which shows that the PSII fluorescence decrease in state II is not due to homogenous quenching of all PSII complexes. Rather, PSII quenching in state II is a local phenomenon and there is a distribution of quenched and unquenched PSII complexes. Thus, the mechanism of PSII fluorescence quenching in state II concluded for Synechococcus, from the results shown in Fig. 2 is in full agreement with the one hypothesized for Synechocystis from the results shown in Fig. 1. Therefore, in state II, from the pronounced PSIl quenching in Synechococcus $\triangle \mathrm{ApcD}$ (Fig. $1 \mathrm{C}$ ) and the inhibition of quenching in Synechocystis $\Delta \mathrm{ApcD}$ (Fig. 1B and Fig. S1) it cannot be proposed that the mechanism of state transitions is different in both species..

\section{Reorganization of PBS-PSII complexes during state transitions}

The results in Fig. 2 confirm that PSII complexes exhibit structural flexibility for the reversible excited-state quenching during state transitions. However, it is not clear from the experiment shown in Fig. 2 whether reorganization/reorientation of PBS-PSII complexes during state transitions is also involved to (possibly) keep the PSII complexes in an unquenched conformation in state $I$ and in a quenched conformation in state II. In the steady-state fluorescence spectra of $0.4 \mathrm{M} \mathrm{KH}_{2} \mathrm{PO}_{4}$-incubated cells of Synechococcus and Synechocystis a pronounced fluorescence quenching was observed in state I as compared to state II (Fig. 3). This potassium-phosphate-induced quenching is concluded in this work to be unrelated to the photosynthetic activity ( $\mathrm{pH}$ or redox state of the $\mathrm{PQ}$ pool) or some salt-induced chemical activity. Steady-state fluorescence quenching is a standard method/tool to characterize the protein structures and conformation changes [27]. In the presence of $0.4 \mathrm{M} \mathrm{KH}_{2} \mathrm{PO}_{4^{\prime}}$ the PBS-PSIl complexes are very stable and the steadystate fluorescence spectra represent the different configurations in which the PBS-PSII complexes are locked in both states. Therefore, the results shown in Fig. 3, confirm the 
prediction based on the Synechocystis WT and Synechocystis $\triangle \mathrm{ApcD}$ results in Fig. 1, that a reorganization of PBS-PSII complexes occurs during state transitions. This conclusion is consistent with freeze-fracture electron microscopy data which shows that in state I $40 \%$ of the PSII particles are arranged in rows, whereas this number is less than $20 \%$ in state II in Synechocystis $6714{ }^{[9]}$. The organizational changes in the arrangement of PSII particles were also found in Synechocytsis $6803^{[20]}$. The immediate cause of PSIl quenching in state II cannot be determined from the results shown in this chapter. However, it can be proposed that some structural changes in the thylakoid membrane in state II might induce a quenched conformation in an increased population of PSII complexes as compared to state I. This possibility is further discussed in chapter 6 .

\section{Interaction of PBSs and PSII complexes in Synechococcus and Synechocystis}

Fig. 3 shows that potassium-phosphate-induced fluorescence quenching of PBSs is negligible in Synechococcus as compared to Synechocystis. Furthermore, potassiumphosphate-induced fluorescence quenching of PSII complexes is also much more pronounced in Synechocystis as compared to Synechococcus. In fact, in Synechocystis the fluorescence quenching of both PBSs and PSII complexes, increases proportionally as a function of increasing $\mathrm{KH}_{2} \mathrm{PO}_{4}$ concentrations. PBS-PSIl complexes, of Synechocystis state-1 cells incubated with $\mathrm{KH}_{2} \mathrm{PO}_{4}$ are locked in a configuration which is much more susceptible to the potassium-phosphate-induced quenching than those of Synechococcus. Therefore, the results in Fig. 3 confirm the proposition in the hypothesis section that the interaction of PBSs with PSII complexes is different in Synechococcus and Synechocystis.

\section{Conclusions}

A salt-induced quenching method is introduced in this work to probe the interaction of PBSs with photosystems and conformational states of PBSs and photosystems in cyanobacteria in vivo. From the two different species of cyanobacteria, i.e., Synechococcus and Synechocystis it is found that in state II an increased amount of PSII complexes become quenched as observed both upon PBS and $\mathrm{Chl} a$ excitation. The structural flexibility of the core antenna complexes allows them to switch conformation from unquenched in state I to quenched in state II. Transient interactions of PBSs and PSII complexes ensures that PSII complexes are transiently free to change their orientation in the membrane and undergo conformational changes during state transitions. PBS-PSII complexes, locked in two different configurations in state I and state II, show that PBS mobility is not related to modulation of the PSII antenna size during state transitions. Different observations regarding state transitions in Synechocystis WT, Synechocystis $\triangle \mathrm{ApcD}$, and Synechococcus $\triangle \mathrm{ApcD}$ are mere manifestations of some difference in the interaction details of PBSs and PSIl complexes between Synechococcus and Synechocystis. 


\section{References}

1. Bellafiore, S., et al., State transitions and light adaptation require chloroplast thylakoid protein kinase STN7. Nature, 2005. 433(7028): p. 892-895.

2. Lemeille, S., et al., Analysis of the chloroplast protein kinase Stt7 during state transitions. PLoS biology, 2009. 7(3): p. e1000045.

3. Allen, J.F., Protein phosphorylation in regulation of photosynthesis. Biochimica et Biophysica Acta (BBA)-Bioenergetics, 1992. 1098(3): p. 275-335.

4. Wientjes, E., H. van Amerongen, and R. Croce, LHCIl is an antenna of both photosystems after long-term acclimation. Biochimica et Biophysica Acta (BBA) - Bioenergetics, 2013. 1827(3): p. 420-426.

5. Ünlü, C., et al., State transitions in \&lt;em\&gt;Chlamydomonas reinhardtii\&/t;/em\&gt; strongly modulate the functional size of photosystem II but not of photosystem I. Proceedings of the National Academy of Sciences, 2014. 111(9): p. 3460.

6. Joshua, S. and C.W. Mullineaux, Phycobilisome Diffusion Is Required for Light-State Transitions in Cyanobacteria. Plant Physiology, 2004. 135(4): p. 2112.

7. Arteni, A.A., et al., Structure and organization of phycobilisomes on membranes of the red alga Porphyridium cruentum. Photosynthesis Research, 2008. 95(2): p. 169-174.

8. Chang, L., et al., Structural organization of an intact phycobilisome and its association with photosystem II. Cell research, 2015. 25(6): p. 726-737.

9. Olive, J., et al., Randomization of the EF particles in thylakoid membranes of Synechocystis 6714 upon transition from state I to state II. FEBS letters, 1986. 208(2): p. 308-312.

10. Mullineaux, C.W. and J.F. Allen, Fluorescence induction transients indicate dissociation of photosystem II from the phycobilisome during the state-2 transition in the cyanobacterium Synechococcus 6301. Biochimica et Biophysica Acta (BBA)-Bioenergetics, 1988. 934(1): p. 96107.

11. Sanders, C.E. and J.F. Allen, The 18.5 kDa phosphoprotein of the cyanobacterium Synechococcus 6301: A component of the phycobilisome, in Progress in Photosynthesis Research. 1987, Springer. p. 761-764.

12. Allen, J.F. and N.G. Holmes, A general model for regulation of photosynthetic unit function by protein phosphorylation. FEBS letters, 1986. 202(2): p. 175-181.

13. $\mathrm{Li}, \mathrm{H}$. , et al., The state transition mechanism — simply depending on light-on and-off in Spirulina platensis. Biochimica et Biophysica Acta (BBA)-Bioenergetics, 2006. 1757(11): p. 1512-1519.

14. Li, D., et al., Light-induced excitation energy redistribution in Spirulina platensis cells: "spillover" or "mobile PBSs"? Biochimica et Biophysica Acta (BBA)-Bioenergetics, 2004. 1608(2-3): p. 114121.

15. Mullineaux, C.W., Excitation energy transfer from phycobilisomes to Photosystem I in a cyanobacterium. Biochimica et Biophysica Acta (BBA) - Bioenergetics, 1992. 1100(3): p. 285292. 
16. Bald, D., J. Kruip, and M. Rögner, Supramolecular architecture of cyanobacterial thylakoid membranes: How is the phycobilisome connected with the photosystems? Photosynthesis Research, 1996. 49(2): p. 103-118.

17. Bruce, D., et al., Mechanism of the light state transition in photosynthesis. IV. Picosecond fluorescence spectroscopy of Anacystis nidulans and Porphyridium cruentum in state 1 and state 2 at 77 K. Biochimica et Biophysica Acta (BBA)-Bioenergetics, 1985. 806(2): p. 237-246.

18. Olive, J., et al., Ultrastructure and light adaptation of phycobilisome mutants of Synechocystis PCC 6803. Biochimica et Biophysica Acta (BBA)-Bioenergetics, 1997. 1319(2-3): p. 275-282.

19. Bruce, D., S. Brimble, and D.A. Bryant, State transitions in a phycobilisome-less mutant of the cyanobacterium Synechococcus sp. PCC 7002. Biochimica et Biophysica Acta (BBA)Bioenergetics, 1989. 974(1): p. 66-73.

20. Olive, J., et al., Ultrastructure and light adaptation of phycobilisome mutants of Synechocystis PCC 6803. Biochimica et Biophysica Acta (BBA) - Bioenergetics, 1997. 1319(2): p. 275-282.

21. Folea, I.M., et al., Domain organization of photosystem II in membranes of the cyanobacterium Synechocystis PCC6803 investigated by electron microscopy. FEBS Letters, 2008. 582(12): p. 1749-1754.

22. Vernotte, C., C. Astier, and J. Olive, State 1-state 2 adaptation in the cyanobacteria Synechocystis PCC 6714 wild type and Synechocystis PCC 6803 wild type and phycocyanin-less mutant. Photosynthesis Research, 1990. 26(3): p. 203-212.

23. El Bissati, K., et al., Photosystem II fluorescence quenching in the cyanobacterium Synechocystis PCC 6803: involvement of two different mechanisms. Biochimica et Biophysica Acta (BBA) Bioenergetics, 2000. 1457(3): p. 229-242.

24. Ley, A. and W. Butler, Energy distribution in the photochemical apparatus of Porphyridium cruentum in state I and state II. Biochimica et Biophysica Acta (BBA)-Bioenergetics, 1980. 592(2): p. 349-363.

25. Acuña, A.M., et al., Resolving the contribution of the uncoupled phycobilisomes to cyanobacterial pulse-amplitude modulated (PAM) fluorometry signals. Photosynth Res, 2016. 127(1): p. 91-102.

26. Farooq, S., et al., Dynamic feedback of the photosystem II reaction centre on photoprotection in plants. Nature Plants, 2018. 4(4): p. 225-231.

27. Mátyus, L., J. Szöllősi, and A. Jenei, Steady-state fluorescence quenching applications for studying protein structure and dynamics. Journal of Photochemistry and Photobiology B: Biology, 2006. 83(3): p. 223-236. 


\section{Supplementary Information}

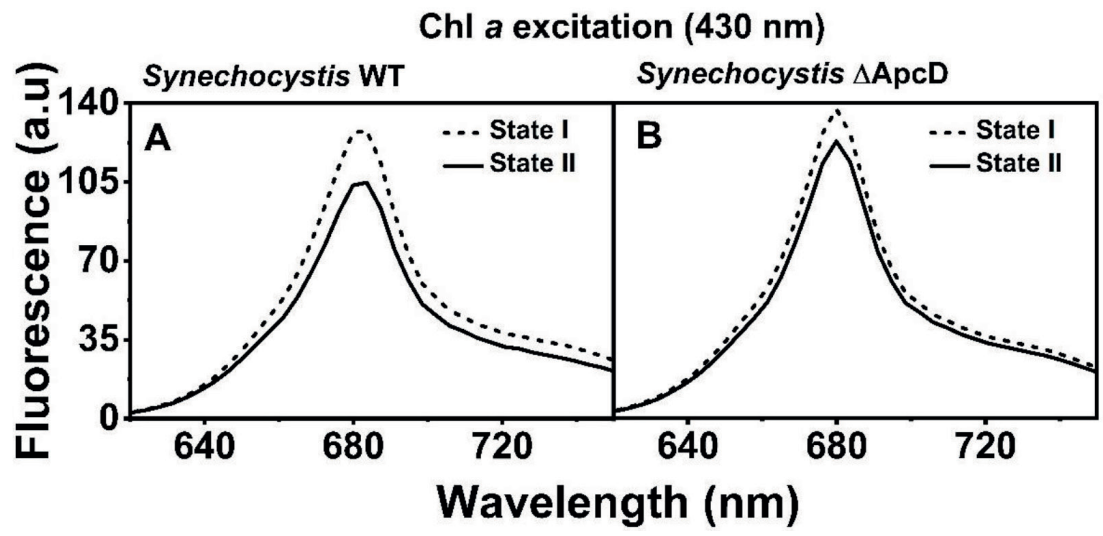

Fig. S1. Reconstructed steady-state fluorescence of (A) Synechocystis WT cells in state I (dotted line) and state II (solid line), (B) Synechocystis $\triangle \mathrm{ApcD}$ cells in state I (dotted line) and state II (solid line).

$\mathrm{KH}_{2} \mathrm{PO}_{4}$-induced quenching in state-I cells PBS Excitation (577 nm)

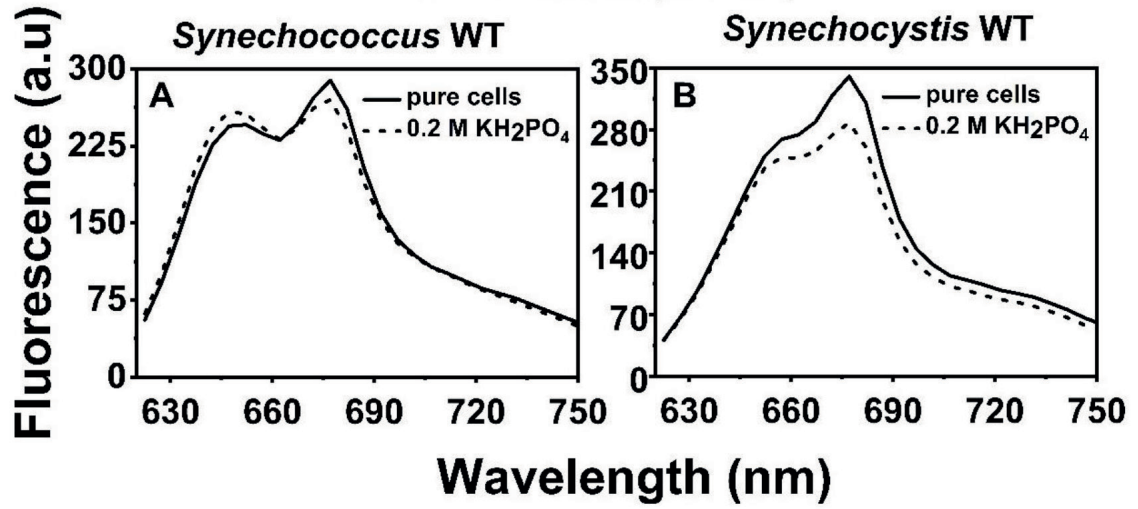

Fig. S2. Reconstructed steady-state fluorescence of (A) pure Synechococcus WT cells (solid line) and $0.2 \mathrm{M}$ $\mathrm{KH}_{2} \mathrm{PO}_{4}$-incubated cells (broken line) in state I (in blue light).(B) Reconstructed steady-state fluorescence in state I (in blue light) of pure Synechocystis WT cells (solid line) and $0.2 \mathrm{M} \mathrm{KH}_{2} \mathrm{PO}_{4}$-incubated cells (broken line). 


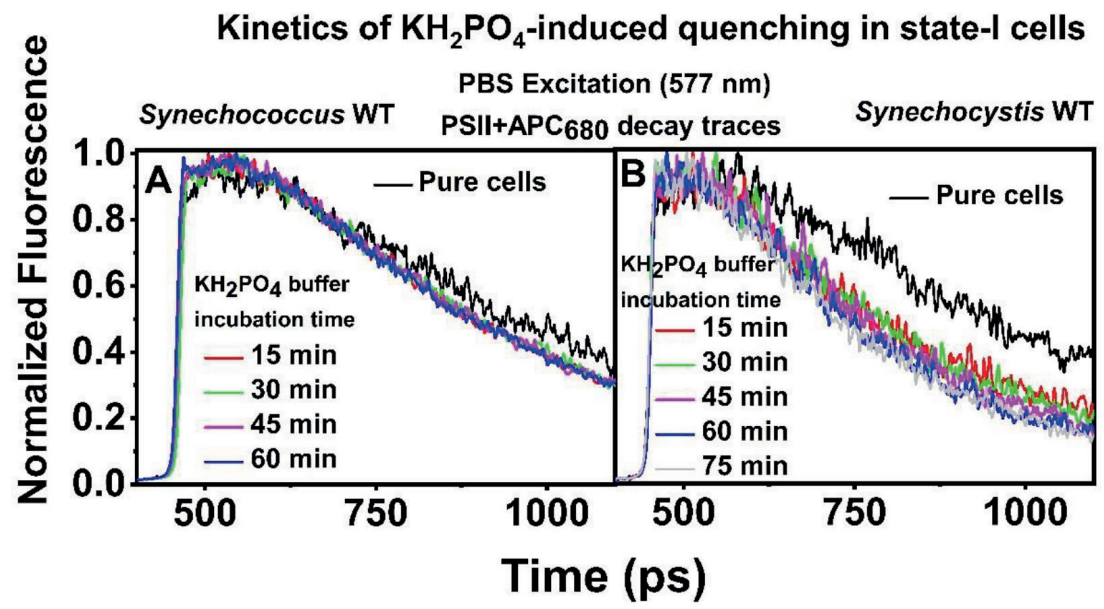

Fig. S3. Kinetics of potassium-phosphate-induced quenching in state-I cells of (A) Synechococcus and (B) Synechocystis. (A) Fluorescence decay traces recorded at $\sim 680 \pm 5 \mathrm{~nm}$ (PSIl and APC $\mathrm{Gro}_{60}$ emission maxima) upon PBS excitation ( $577 \mathrm{~nm}$ ) from WT cells of (A) Synechococcus and (B) Synechocystis. Black line in both cases represents fluorescence decay from pure cells. $\mathrm{KH}_{2} \mathrm{PO}_{4}(0.4 \mathrm{M})$ was then added to the pure cells and decay traces were recorded as a function of salt-incubation time (15-75 minutes) as represented by colored lines in both, $A$ and $B$.

Incubation with potassium phosphate (0.4) resulted in fluorescence quenching at $\sim 680$ $\mathrm{nm}$ (PSII and $\mathrm{APC}_{680}$ ) in both, Synechococcus and Synechocystis (Fig. S3). In Synechococcus the potassium-phosphate-induced quenching is very similar from 15 minutes (red line) to 60 minutes incubation times (blue line). Although in Synechocystis cells a significant amount of salt-induced quenching can be observed after 15 minutes of incubation, the full effect of the salt on fluorescence quenching is reached after $\sim 60$ minutes. Both Synechococcus and Synechocystis were fully equilibrated in state I prior to the addition of $\mathrm{KH}_{2} \mathrm{PO}_{4}$ and measurements were performed at $\sim 7.5 \mathrm{pH}$. These conditions rule out the possibility that the difference in the kinetics and amount of salt-induced quenching between both species could be due to differences in photosynthetic activity and/or variation in membrane-surface charges. It was shown in the previous study ${ }^{[6]}$ that the state transitions related fluorescence changes in Synechococcus were fully reversible upon extraction of salt from salt-incubated cells. Therefore, the salt-induced quenching and the difference in the kinetics and strength of quenching between both species as observed in Fig. S3 cannot be ascribed to some chemical reaction of salt in cells. All the measurements in Fig. 3 were performed after complete incubation of the cells with the buffer. 


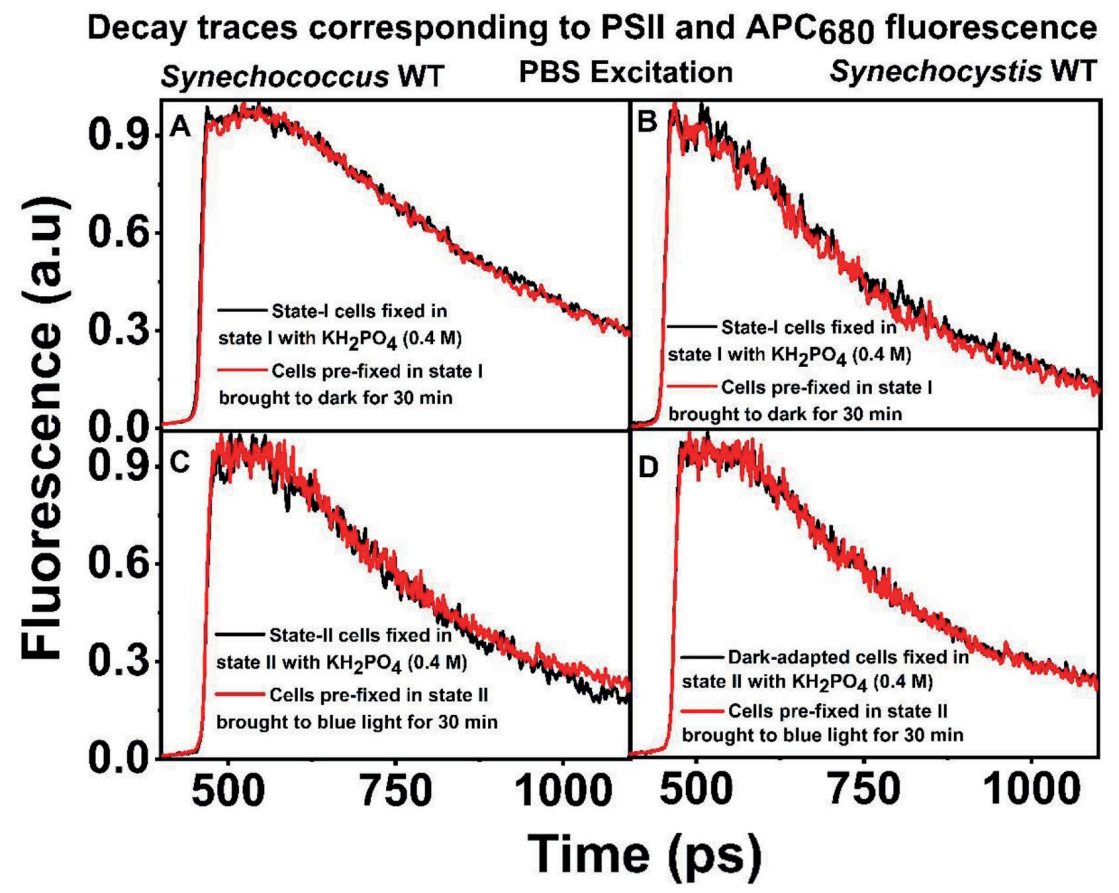

Fig. S4. Fluorescence decay traces recorded at $\sim 680 \pm 5 \mathrm{~nm}$ (PSIl and $A P C_{680}$ emission maxima) upon PBS excitation from WT cells of (A, C) Synechococcus and (B, D) Synechocystis. (A, B), black line represents decay trace from cells in state I, kept in blue light and incubated in $\mathrm{KH}_{2} \mathrm{PO}_{4}(0.4 \mathrm{M})$ buffer to fix cells in the same state. Cells priorly fixed in state I (with $\mathrm{KH}_{2} \mathrm{PO}_{4}$ buffer) were later adapted to dark for $\sim 30$ minutes and fluorescence decay was recorded (red line in both, A and B). (C, D), black line represents decay trace from cells in state II, kept in dark and incubated in $\mathrm{KH}_{2} \mathrm{PO}_{4}(0.4 \mathrm{M})$ buffer to fix cells in the same state. Cells priorly fixed in state II (with $\mathrm{KH}_{2} \mathrm{PO}_{4}$ buffer) were later adapted to light for $\sim 30$ minutes and fluorescence decay was recorded (red line in both, $C$ and $D$ ).

In Synechocystis WT the amount of salt-induced quenching is much stronger in state I cells as compared to state II cells (Fig. 3). To check whether the higher quenching in state I is the effect of blue light, first, fluorescence decay from cells fixed in state I (with incubation in $\mathrm{KH}_{2} \mathrm{PO}_{4}$ ) was recorded at $\sim 680 \mathrm{~nm}$ in the presence of blue light for both, Synechococcus and Synechocystis (black lines in Fig. S4(A,B)). The same cells were then adapted to dark for $\sim 30$ minutes and the decay trace was recorded again at $\sim 680 \mathrm{~nm}$ (red lines). Fluorescence decay from cells pre-fixed in state $\mathrm{I}$, was found comparable both, in light-adapted and dark-adapted cells (Fig. S4 (A,B)), showing that the higher salt-induced quenching in state $I$ is not the effect of blue light. Fluorescence decay traces recorded from cells prefixed in state II (with incubation in $\mathrm{KH}_{2} \mathrm{PO}_{4}$ ) were also found to be comparable, both from dark-adapted and dark-to-light-adapted cells (Fig. S4(C, D)). Therefore from Fig. S4 it is concluded that salt-induced quenching is in principle independent of dark/light state and the redox state of the plastoquinone pool. 


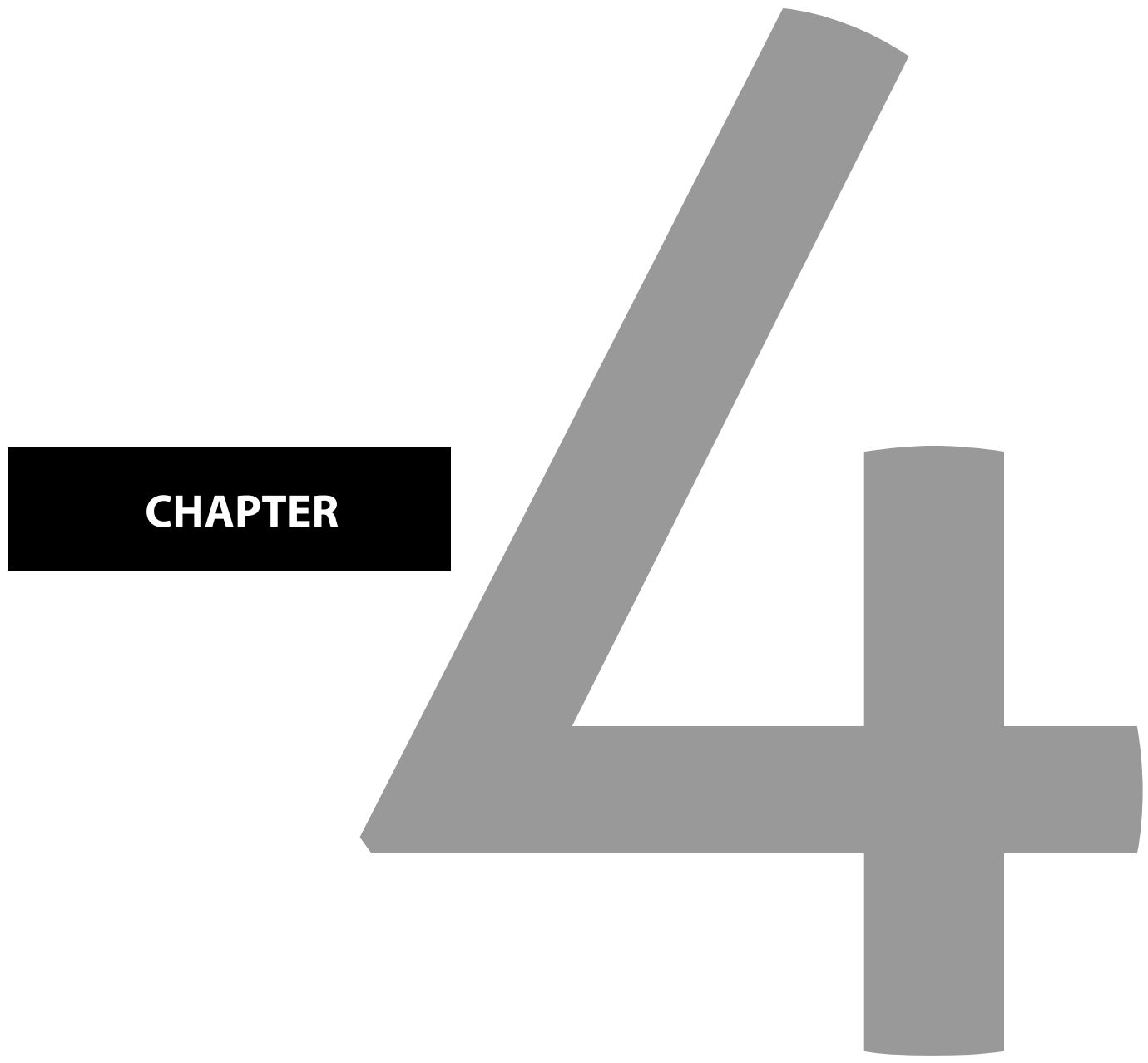




\section{State transitions and photosystems spatially resolved in individual cells of the cyanobacterium Synechococcus elongatus}

Ahmad Farhan Bhatti, Diana Kirilovsky, Herbert van Amerongen, Emilie Wientjes 
State transitions are a low-light acclimation response through which the excitation of Photosystem I (PSI) and Photosystem II (PSII) is balanced; however, our understanding of this process in cyanobacteria remains poor. Here, picosecond fluorescence kinetics were recorded for the cyanobacterium Synechococcus elongatus using fluorescence-lifetime-imaging microscopy (FLIM), both upon chlorophyll $a$ and phycobilisome (PBS) excitation. Fluorescence kinetics of single cells obtained using FLIM were compared with those of ensembles of cells obtained with time-resolved fluorescence spectroscopy. The global distribution of photosystems I (PSI) and II (PSII) and PBSs was mapped making use of their fluorescence kinetics. Both radial and lateral heterogeneity was found in the distribution of the photosystems. State transitions ware studied at the level of single cells. FLIM results show that PSII quenching occurs in all cells, irrespective of their state (I or II). In S. elongatus cells this quenching is enhanced in state II. Furthermore, the decrease of the PSII fluorescence in state II was found to be homogeneous throughout the cells, despite the inhomogeneous PSI/PSII ratio. Finally, some disconnected PBSs were resolved in most state-II cells. Taken together our data shows that PSI is enriched in the inner thylakoid(s), while state transitions occur homogeneously throughout the cell. 


\section{Introduction}

Virtually all life in our biosphere is sustained by sunlight which is harvested and transformed into chemical energy by the process of photosynthesis. In oxygenic photosynthesis Photosystem I (PSI) and Photosystem II (PSII) are embedded in the thylakoid membrane and work in series to drive the electron transport from water to NAD $(P)^{+}$. Photosynthetic organisms are subject to various types of light stress due to ever changing illumination conditions (in terms of duration, intensity and spectral composition). In response to light stress, photosynthetic organisms have developed strategies to avoid photodamage and/ or to maintain the optimal rate of photochemistry. State transitions form a low-light acclimation response by which plants, algae and cyanobacteria balance the excitation of PSI and PSII. The light that preferentially excites PSI, leads to oxidation of the plastoquinone pool and drives the photosynthetic system to state I ${ }^{[1,2]}$. The light that preferentially excites PSII, reduces the plastoquinone pool and drives the photosynthetic system to state II ${ }^{[1,2]}$.

In green plants state transitions occur via the redistribution of light-harvesting complexes II (LHCII) between PSI and PSII ${ }^{[3-6]}$. In state I, LHCII is closely associated with PSIl and transfers most of the absorbed energy to PSII ${ }^{[3,4]}{ }^{4}$ In state II, the reduction of the plastoquinone pool results in phosphorylation and decoupling of a proportion of LHCII from PSII and its association with PSI ${ }^{[3,4]}$. In cyanobacteria phycobilisomes (PBSs) are considered to be the functional equivalents of LHCII in plants. Despite the fact that state transitions in cyanobacteria were already discovered in $1969^{[7]}$ a comprehensive understanding of the process is still lacking.

State transitions are characterized by a decrease in the fluorescence of PSII in state II ${ }^{8-}$ ${ }^{10]}$. In cyanobacteria this is observed when probed with light that is selectively absorbed by either PBSs or chlorophyll (Chl) $a^{[11-13]}$. A strong decrease of PSIl fluorescence in state II observed upon Chl $a$ excitation cannot be explained by the redistribution of PBSs between PSII and PSI ${ }^{[10,14]}$. This immediately distinguishes the process of state transitions in cyanobacteria from that in plants. Generally, the proposed mechanism for state transitions in cyanobacteria has been conceptually similar to the mechanism in plants and green algae. An increase of the excitation-energy transfer (EET) to PSI at the expense of PSII in state II was suggested to occur in plants and green algae ${ }^{[3-6]}$. In cyanobacteria the proposed reduced EET to PSII reaction centers in state II was explained by different hypotheses (i) (Re)Distribution of PBSs between PSII and PSI in Synechococcus 6301 was concluded to regulate the PBS-absorbed excitation energy [15]; (ii) The fluorescence decrease in state II in the cyanobacteria Anacystis nidulans and Synechocystis PCC 6803 was ascribed to the direct spillover of excitation energy from PSII to PSI ${ }^{[16,17]}$; (iii) In ${ }^{[10]}$ it was proposed that in a megacomplex of PBS-PSI-PSII a decreased distance in photosystems in state II would result both in increased transfer of excitation energy from PBS to PSI and 
spillover of excitation energy from PSII to PSI in Synechococcus 7002 and Synechocystis 6803. However, in none of the above-mentioned studies, the expected increase of PSI fluorescence was observed in state II at the expense of PSII fluorescence. On the contrary, in plants an increase in the PSI fluorescence at the expense of PSII fluorescence in state II is clearly observed using time-resolved fluorescence spectroscopy ${ }^{[18]}$. This casts doubt whether a redistribution of excitation energy between PSI and PSII occurs during state transitions in cyanobacteria. In ${ }^{[12]}$, state transitions in Synechococcus elongatus were induced at RT and by rapidly freezing the various states were "captured/stabilized" and studied at 77K. No evidence was found for the migration of PBSs from PSII to PSI in state II nor for the spillover of energy from PSII to PSI. Instead the observed decrease in PSII fluorescence in state II was ascribed to direct quenching of PSII complexes. However, it is still not clear if reversible physical changes in the association of PBSs and PSII are involved in the process which leads to quenching of PSII in state II. The role of PBSs in state transitions is particularly ambiguous, since in Synechocystis 6803, Synechococcus 7002 and Synechococcus 7120, the mutants lacking a terminal emitter protein named ApcD show no fluorescence changes upon PBS excitation and a reduced PSII fluorescence quenching upon $\mathrm{Chl} a$ excitation in state II ${ }^{[13,19-22]}$. In our recent work ${ }^{[13]}$, it was indeed shown that in Synechocystis 6803 the PSII fluorescence decrease in state II observed upon photosystems excitation ( $\mathrm{Chl}$ ), was only $\sim 9 \%$ in the Synechocystis $\triangle \mathrm{ApcD}$ as compared to $21 \%$ for WT cells. This suggests that PBSs are somehow involved in the process of state transitions in cyanobacteria.

Time-resolved fluorescence spectroscopy can in principle distinguish between the different scenarios discussed above to explain the decrease in the PSII fluorescence in state II. However, this approach usually provides an average over a very large ensemble of cells. Thus, the information about possible inhomogeneities in the spatial distribution of photosynthetic complexes in cyanobacteria cells is inaccessible with this method. In recent years, microscopy studies using either hyperspectral imaging ${ }^{[23]}$ or labelling with fluorescence proteins ${ }^{[24,25]}$ have shown that PSI, PSII and PBSs are inhomogeneously distributed over the thylakoid membranes of cyanobacteria. In a fluorescence lifetime imaging microscopy (FLIM) study on Synechocystis 6803 cells, disconnected PBSs were observed in a fraction of the cells and large variations regarding the disconnection of PBSs were found between cells ${ }^{[26]}$. In line with these results it appears very purposeful to study state transitions by mapping the fluorescence kinetics at the sub-cellular level in living cyanobacteria. To our knowledge any such study is lacking so far.

In the present work we used confocal FLIM to study state transitions in Synechococcus elongatus cells at room temperature. These cells have a cylindrical shape, with typical dimensions of $\sim 2.5 \mu \mathrm{m}^{*} 1.1 \mu \mathrm{m}^{*} 1.1 \mu \mathrm{m}{ }^{[27]}$. The thylakoid membrane is located near the periphery of the cell and consists typically of 4 membrane layers, which are connected 
and enclose a single lumen ${ }^{[28]}$. To investigate the spatial distribution of PBSs, PSI and PSII in the thylakoid membrane and their role in state transitions, selective excitation of PBSs and $\mathrm{Chl} a$ was used. The results were compared with ensemble time-resolved fluorescence measurements which we reported earlier in ${ }^{[13]}$.

\section{Materials and Methods}

\section{Sample Preparation}

Synechococcus PCC 7942 (S. elongatus) cells were grown in BG-11 medium (20 ml/l) supplemented with sodium bicarbonate $(0.85 \mathrm{~g} / \mathrm{l})$ and sodium nitrate $(1.75 \mathrm{~g} / \mathrm{l})$ at $\mathrm{pH}$ $\sim 8.0$. Cells were grown at $30^{\circ} \mathrm{C}$ under white light illumination at $50 \mu \mathrm{mol}$ photons $\mathrm{m}^{-2} \mathrm{~s}^{-1}$ in $250 \mathrm{ml}$ flasks shaken at $100 \mathrm{rpm}$, containing culture volumes of $60 \mathrm{ml}$ as described in ${ }^{[12]}$. Cells in the logarithmic phase of growth at optical density $\sim 0.6-0.7$ at $800 \mathrm{~nm}\left(O D_{800}\right.$ for a path length of $1 \mathrm{~cm}$ ) were harvested for the measurements. Harvested cells were then centrifuged at $2500 \mathrm{rpm}$ for 10 minutes and immobilized on agar coated microscopic slides ( 2\% invitrogen select agar mixed with growth medium). Immediately prior to the measurements, microscopic slides were illuminated for 30 minutes with blue light $\left(438 \pm 22 \mathrm{~nm}\right.$ at $\sim 40 \mu \mathrm{mol}$ photons $\left.\mathrm{m}^{-2} \mathrm{~s}^{-1}\right)$ to induce state I. Cells adapted to state I were kept under the same light condition for the duration of the measurement as in ${ }^{[13]}$. The combined sample handling and measurement time was less than 5 minutes. Afterwards, the same slides were kept in darkness for 30 minutes to induce state II and the same cells were measured.

\section{FLIM measurements}

Measurements were performed on an (inverted) confocal Leica TCS SP8 Hyd fluorescence microscope. A $63 \times 1.20$ NA water immersion objective was used to image the cells. The confocal pinhole diameter of the microscope was between 0.5-0.8 AU. The lateral and the axial resolution of the microscope were $\sim 0.2 \mu \mathrm{m}$ and $\sim 0.6 \mu \mathrm{m}$, respectively. Chl $a$ was excited at $440 \mathrm{~nm}$ using a diode pulsed laser and PBSs were excited at $577 \mathrm{~nm}$ using a pulsed super continuum laser. At $440 \mathrm{~nm}$ excitation $0.025 \mu \mathrm{W}$ laser power was used. At $577 \mathrm{~nm}$ excitation $0.010 \mu \mathrm{W}$ laser power was used. The laser repetition rate in both cases was $40 \mathrm{MHz}$ and the scan rate was $400 \mathrm{~Hz}$. Fluorescence was detected at $685-720 \mathrm{~nm}$ with internal hybrid detectors coupled to time-correlated single photon counting (TCSPC) boards (SPC-152, Becker \& Hickl GmbH), as described in ${ }^{[29]}$. Images were recorded for 60 sec upon $\mathrm{Chl} a$ excitation and for $20 \mathrm{sec}$ upon PBS excitation. The total image size was either $48 \mu \mathrm{m}^{*} 48 \mu \mathrm{m}$ or $29 \mu \mathrm{m}^{*} 29 \mu \mathrm{m}$ with $256^{*} 256$ pixels. The time step for the TCSPC detection was $12 \mathrm{ps} /$ channel.

FLIM images were analyzed using image analysis software (SPClmage, Becker \& Hickl $\mathrm{GmbH}$ ). Pixel binning of 5 or higher (maximum 8 ) was used. A set of very reproducible 
lifetimes for different cells and different biological samples was found from the global analysis of FLIM images. Pinacyanol chloride dissolved in methanol with lifetime of $6 \mathrm{ps}$ ${ }^{[30]}$ was used to measure the instrument response function for the FLIM measurements. All measurements were performed at room temperature.

\section{Results}

\section{Fluorescence lifetime imaging of Synechococcus elongatus}

The aim of this work is to refine the understanding of the state-transitions process in cyanobacteria by investigating the role of PSI, PSII and PBSs at the cellular level using FLIM. For this purpose, the cells were probed in state I and state II after selective excitation of photosystems (440 nm excitation) and PBSs (577 nm excitation). All measurements were performed at room temperature. State I was induced by keeping the cells for 30 minutes in blue light. State II was induced by keeping the cells in darkness. The same cells were imaged for State I and State II. respectively. The state-I cells were also illuminated with blue light during the measurement time of 60 seconds. The fluorescence kinetics at the cellular level obtained in this work are compared with the fluorescence kinetics of an ensemble of S. elongatus cells reported earlier ${ }^{[13]}$.

\section{Experimental conditions for FLIM}

Direct excitation of the photosystems at $440 \mathrm{~nm}$ allowed us to image the fluorescence kinetics of PSI and PSII from S. elongatus cells in state I and state II. $577 \mathrm{~nm}$ excitation was used to probe the interaction of PBSs with photosystems in different states. The timeresolved fluorescence was recorded in the $685 \mathrm{~nm}-720 \mathrm{~nm}$ range, where both PSI and PSII have significant emission.

Our previous time-resolved spectroscopic studies at room temperature and 77K have revealed that state transitions in cyanobacteria are characterized by a reversible decrease in the average PSII fluorescence lifetime in state II ${ }^{[11-13]}$. However, the fluorescence kinetics of PSII is also influenced by the laser light intensity. At very low excitation intensities, due to photochemical quenching of excitations by open PSII reaction centers, PSII fluorescence shows faster kinetics ${ }^{[31]}$. At relatively high light intensities, the PSII fluorescence kinetics becomes slower due to the closure of PSII reaction centers ${ }^{[31]}$. The PSI fluorescence kinetics are equally fast ( $20 \mathrm{ps}$ ) for open and closed RCs and they are thus independent of the light intensity ${ }^{[32]}$. In this work all measurements were done with closed PSII reaction centers to completely separate the PSI and PSII fluorescence kinetics from each other. In laser scanning confocal microscopy a diffraction limited laser spot scans the sample to obtain a fluorescence based image. An accurate determination of the excitation scanning parameters is essential to reliably image the state transitions in individual cells of cyanobacteria. On the one hand the laser intensity should be high enough to close 
the PSII reaction centers. On the other hand the laser intensity should be low enough to avoid singlet-singlet and singlet-triplet annihilation, which give rise to additional decay pathways. Furthermore, the dose of laser light that the cells receive should be limited to avoid photodamage, which would lead to the creation of quenchers and as such would shorten the excited-state lifetime ${ }^{[29]}$. The control experiments performed to find the correct illumination conditions are described in Fig. S1 and Fig. S2 for both $440 \mathrm{~nm}$ and $577 \mathrm{~nm}$ excitation, respectively. Based on these measurements the $440 \mathrm{~nm}$ and $577 \mathrm{~nm}$ laser light intensities were selected which close the PSII RCs, do not lead to photodamage during the measurement time and allow for reversible state transitions.

\section{Results after Chl $a$ excitation}

Fluorescence intensity and fluorescence lifetime images upon $440 \mathrm{~nm}$ excitation were recorded (Fig. 1) for the same S. elongatus cells first brought to state I (30 min blue light) and next to state II (30 min darkness). As the thylakoid membrane of S. elongatus cells are aligned with the cell wall most of the fluorescence is measured at the periphery of the cells, while the inner regions of the cells show very little fluorescence. State I cells (Figure 1A) show significantly higher fluorescence as compared to state II cells (Figure 1C). Fluorescence decays were recorded using the TCSPC method for each pixel and three lifetimes ( $\boldsymbol{\tau}_{1}=\sim 20-25 \mathrm{ps}, \boldsymbol{\tau}_{2}=\sim 170-180 \mathrm{ps}$ and $\boldsymbol{\tau}_{3}=\sim 700-900 \mathrm{ps}$ ) were sufficient to

\section{S. elongatus}

\section{$440 \mathrm{~nm}$ Excitation}
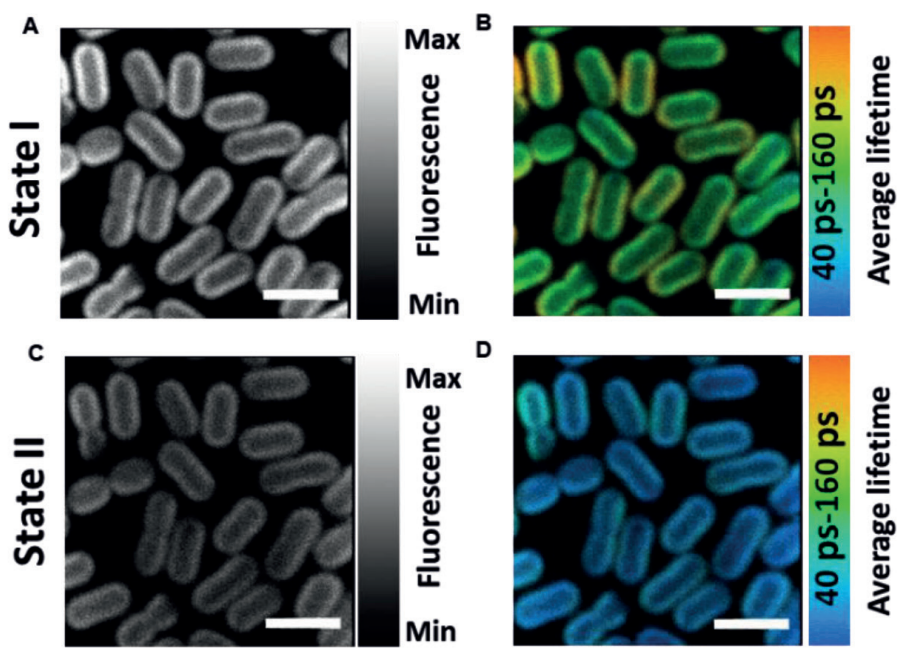

Figure 1. Confocal fluorescence microscope images of $S$. elongatus cells adapted to sate I $(A, B)$ and, state II (C,D). $A, C$ show steady-state intensity images of cells in state I and state II respectively. $(C, D)$ show average lifetime distributions in state I and state II adapted cells. Same cells were imaged for both state I and State II measurements. All images are equally adjusted for brightness and contrast. Excitation wavelength was $440 \mathrm{~nm}$ and fluorescence was recorded in $685-720 \mathrm{~nm}$ range. Measurements were done at room temperature. Scale bar is $4 \mu \mathrm{m}$. See supplemental figure $\mathrm{S} 6$ for reproducibility of state I and II observed upon selective excitation of Chl $a$. 
fit the fluorescence decay across the whole image. Values of these lifetimes are in good agreement with the ones determined from the measurements on the bulk of cells using a streak camera (Figures S3). The short lifetime component ( $\boldsymbol{\tau}_{1}=\sim 20-25 \mathrm{ps}$ ) corresponds to the kinetics of PSI excitation trapping (Fig. S3; ${ }^{[13,32]}$ ). The other two lifetimes $\left(\boldsymbol{\tau}_{2}=\sim 170\right.$ $180 \mathrm{ps}$ and $\boldsymbol{\tau}_{3}=\sim 700-900 \mathrm{ps}$ ) represent the biphasic fluorescence decay of PSII with closed reaction centres (Fig. S3 and ${ }^{[13,31]}$. Figures 1B and 1D show the distribution of the average lifetime for cells in state I and state II, respectively. Cells in state II show a shorter average fluorescence lifetime (Fig. 1D) than cells in state I (Fig. 1B) which is consistent with the lower amount of steady-state fluorescence from state II cells in Fig. 1C.

The amplitude of each lifetime components reflects the relative contribution of the component to the total fluorescence decay. Amplitudes associated with the obtained lifetimes are shown in Figures $2(A, B, C)$ for cells in state $I$ and in Figures $2(D, E, F)$ for cells in state II. The $\sim 20$ ps component, representing the fluorescence kinetics of PSI, has the largest amplitude and contributes $\sim 80 \%$ to the total decay in both states (Figures $2(A, D)$ ).

Characterization of state II using FLIM can reveal if there is a relation between the PSI/ PSII ratio and quenching of PSII. In five cells (i-v) in state II, enclosed in white rectangles in Fig. 2(D-F) a profile of PSI distribution and PSIl quenching was acquired across the width of the cells (Fig. 2(G,H) and Fig. S5). The profiles of PSI distribution and PSIl quenching were acquired in the same way as the thylakoids fluorescence was mapped across the short axis of the Synechococcus cells in ${ }^{[33]}$. Fig. $2 \mathrm{G}$ shows an increase in the percentage distribution of PSI from the periphery towards the centre of the cell across three lines along the short axis of one such representative cell. However, Fig. $2 \mathrm{H}$ shows a nearly homogeneous distribution of quenched PSII/total PSII along the same three lines as in Fig. 2G. Thus the comparison of Fig. $2 \mathrm{G}$ and $2 \mathrm{H}$ shows that the increased quenching of PSII in state II is independent of the PSI distribution (see Fig. S5 for the analysis of 4 different cells). It must be noted that the central region of the cyanobacteria is free of thylakoids and therefore no fluorescence is emitted from this region. However, cyanobacterial cells are cylinderical in shape and the thylakoid membranes follow this shape in the cell (Fig. 2 I). Due to the poor axial resolution of the objective lens $(\Delta z \sim 0.6 \mu \mathrm{m})$, fluorescence will be observed from the inner thylakoids which are present above and below the focal plane of the focal volume. This fluoresence then appears to originate from the center of the cells as depicted in Fig. 2l. This explains the highest amplitude of the a1 component (representing PSI) in the middle of the cells in fig. $2 \mathrm{G}$ and Fig. S5. Fig. S8 (showing analysis at a lower pixel binning) shows that the high amplitude of the a1 component in the middle of the cells is not an artefact of the binning. Schematic in Fig. 21 illustrates that the diffraction limited lateral and axial resolutions of the microscope combined with the pixel binning greatly reduce the observation of PSI heterogeneity between the inner and peripheral thylakoid layers which are spaced by $\sim 80 \mathrm{~nm}$. This means that the modest difference in a1, of 5-10\%, 
that we observe between the outside and the inside of the cell should be caused by a far larger heterogeneity in the PSI distribution.

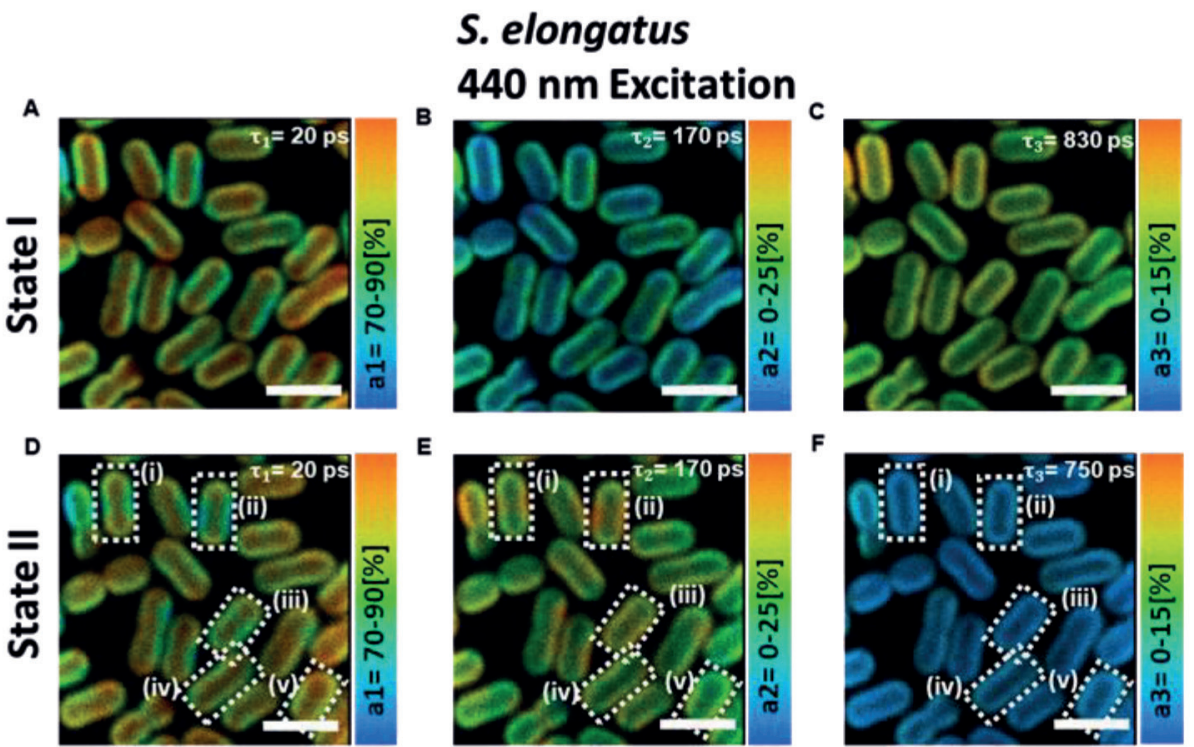

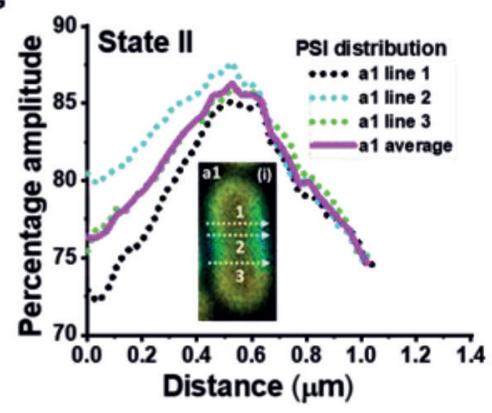

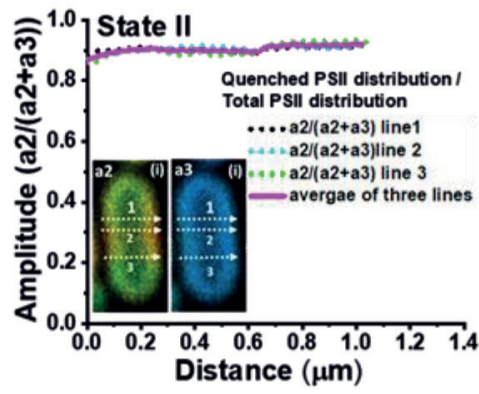

I

Top view: $x, y$ plane

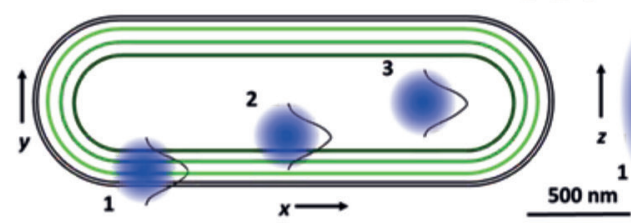

Side view: $\mathbf{z}, y$ plane

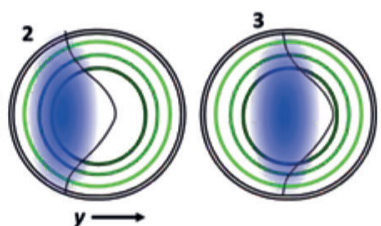

Figure 2. Distribution of amplitudes associated with the fluorescence lifetimes in S. elongatus cells in state I $(A, B, C)$ and state II $(D, E, F)$. Corresponding lifetimes are written in the figures. Excitation wavelength was $440 \mathrm{~nm}$ and fluorescence was detected in $685 \mathrm{~nm}-720 \mathrm{~nm}$ range. Images are adjusted for brightness and contrast. Measurement were done at room temperature. Scale bar is $4 \mu \mathrm{m}$. On five representative cells ( $\mathrm{i}-\mathrm{v}$ ) in state II (lower panel) enclosed in white rectangles, further analysis for state II characterization was performed. (G) shows the profile of amplitude (a1) across three lines along the short axis of the first cell "(i)". (H) shows the distribution of a2/(a2+a3) across the same three lines as in (G). Cell "i" is shown in inset in $\mathrm{G}$ and, $\mathrm{H}$. Analysis on cells "ii-v" is shown in Fig. S5. (I) Schematic representation of the dimensions of the thylakoids (green) in S. elongatus with respect to the confocal observation volume (blue). Three observation volumes are indicated in the $x, y$ and $y, z$ plane. 
Furthermore, in most of the cells there are clearly identifiable regions which show a lower or higher amplitude of the 20 ps (PSI) lifetime component, indicating a lower or higher PSI/ PSII ratio. In Fig. 3A in four cells (i-iv) in state II enclosed in white rectangles, a comparison of PSII quenching (a2/(a2+a3)) was made between the regions of high PSI concentration and low PSI concentration. In Fig. 3(B-E) cells "i" to "iv" are shown, respectively with regions of high PSI content (REG 1 and REG 3) marked with red rectangles and, regions of low PSI content (REG 2 and REG 4) marked with white rectangles. Fig. 3B-E show that the quenching of PSII in state II is similar in the regions of low PSI/PSII ratio and in those of high PSI/PSII ratio in agreement with the results in Fig. 2(G,H).
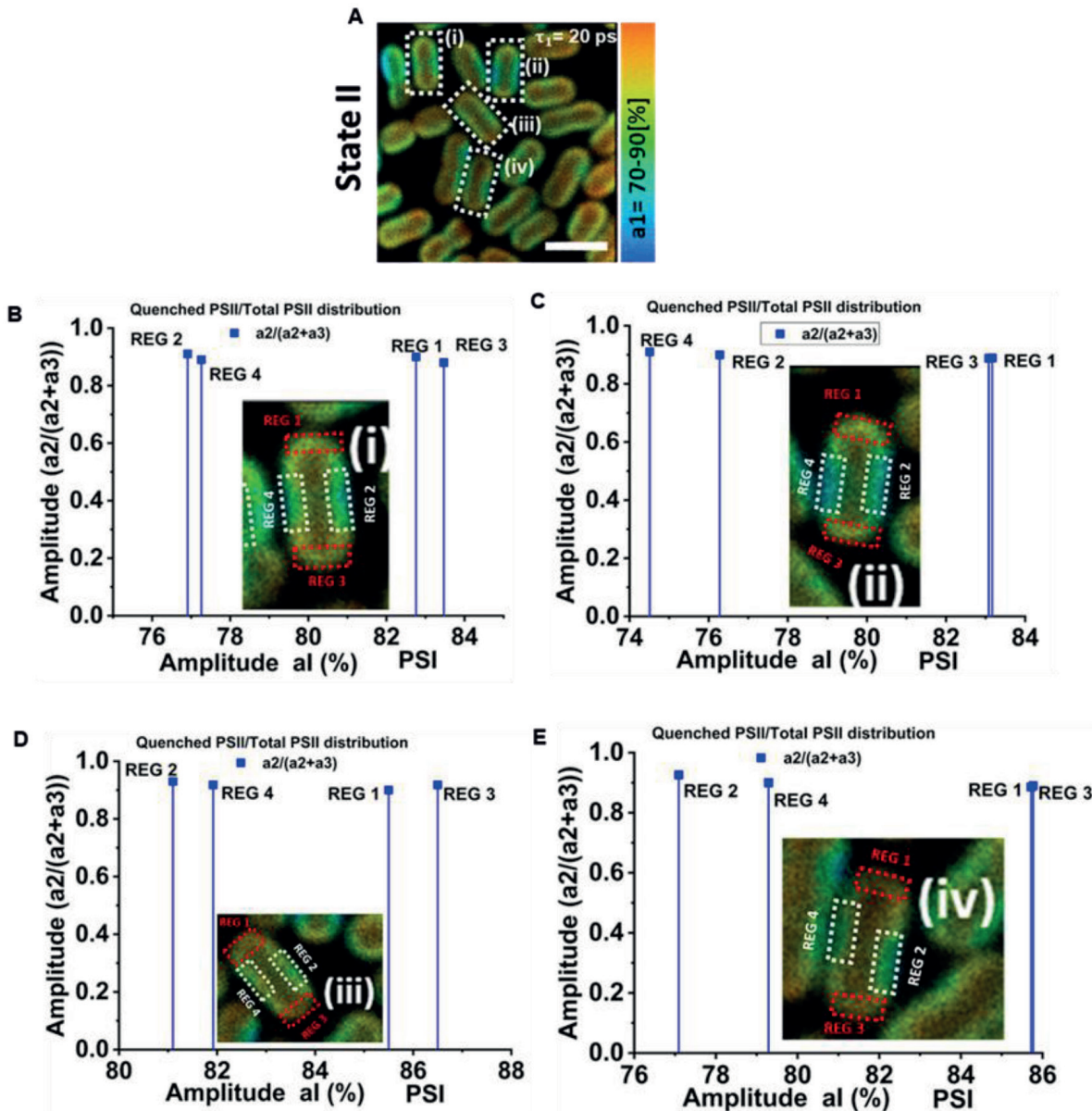

Figure. 3. (A) Distribution of amplitude associated with PSI fluoresence lifetime component (20 ps) in state II cells. On cells "i-iv" marked within white rectangles further analysis was performed to investigate the correlation between the PSI percentage and ratio of quenched PSII/total PSII (a2/(a2+a3)) in specific cell regions. (B, C, D, E) Fraction of quenched PSII of (a2/(a2+a3) vs. the average PSI amplitude in REG 1, REG 2, REG 3 and REG 4 of the cells "i" to "iv", respectively. Cells "i-iv" are shown in inset. "REG 1" and "REG 3" shown with red rectangles represent domains in the cell with higher PSI content. "REG 2" and "REG 4" shown with white rectangles represent domains in the cell with lower PSI content. 
Table I shows the comparison of lifetimes and amplitudes obtained from the analysis of FLIM and streak-camera data in the $685-720 \mathrm{~nm}$ range. The fluorescence decrease in state II as observed in the FLIM measurements was found to be $39.4 \pm 5.1 \%$ (for three biological replicates) which is comparable with the $39.6 \pm 1.1 \%$ decrease observed in streak-camera measurements. The large similarity of the measured values shows that there is a good agreement between the amplitudes resolved with FLIM and streak.

Table 1:

\begin{tabular}{|c|c|c|c|c|c|}
\hline & Lif & components & Amplitudes & State I & State II \\
\hline \multirow{3}{*}{ 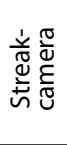 } & $\tau_{1}$ & $\sim 20-25 \mathrm{ps}$ & $\mathrm{A} 1\left(\boldsymbol{\tau}_{1}\right)$ & $80.1 \pm 1.4 \%$ & $79.9 \pm 1.2 \%$ \\
\hline & $\boldsymbol{\tau}_{2}$ & $\sim 180-200 \mathrm{ps}$ & $\mathrm{A} 2\left(\boldsymbol{\tau}_{2}\right)$ & $9.7 \pm 0.6 \%$ & $16.2 \pm 1.1 \%$ \\
\hline & $\tau_{3}$ & $\sim 0.8 \mathrm{~ns}-1.0 \mathrm{~ns}$ & $\mathrm{~A} 3\left(\boldsymbol{\tau}_{3}\right)$ & $10 \pm 0.8 \%$ & $3.7 \pm 0.8 \%$ \\
\hline \multirow{3}{*}{ 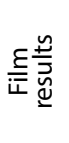 } & $\mathbf{\tau}_{1}$ & $\sim 20 \mathrm{ps}$ & $\mathrm{A} 1\left(\boldsymbol{\tau}_{1}\right)$ & $83.3 \pm 0.7 \%$ & $81.9 \pm 1.9 \%$ \\
\hline & $\boldsymbol{\tau}_{2}$ & $\sim 170-200$ ps & $\mathrm{A} 2\left(\boldsymbol{\tau}_{2}\right)$ & $8.75 \pm 1.3 \%$ & $15.2 \pm 1.8 \%$ \\
\hline & $\tau_{3}$ & $\sim 0.75-0.9 \mathrm{~ns}$ & $\mathrm{~A} 3\left(\boldsymbol{\tau}_{3}\right)$ & $8.9 \pm 1.5 \%$ & $3.4 \pm 1.0 \%$ \\
\hline
\end{tabular}

Comparison of lifetime components and lifetime associated amplitudes obtained from streak-camera measurements and FLIM measurements. $430 \mathrm{~nm}$ and $440 \mathrm{~nm}$ excitation was used for streak-camera measurements and FLIM measurements, respectively. For the streak-camera results, amplitudes A1 $\left(\boldsymbol{\tau}_{1}\right), \mathrm{A} 2\left(\boldsymbol{\tau}_{2}\right)$, and A3 $\left(\boldsymbol{\tau}_{3}\right)$, represent the average percentage contribution of individual amplitudes to the total fluorescence in the $685-720 \mathrm{~nm}$ fluorescence detection range. For the FLIM results, amplitudes $\mathrm{A} 1\left(\boldsymbol{\tau}_{1}\right), \mathrm{A} 2\left(\boldsymbol{\tau}_{2}\right)$, and $\mathrm{A} 3\left(\boldsymbol{\tau}_{3}\right)$, represent the average of the percentage distributions of a1, a2 and, a3, respectively. Average values and standard deviations for amplitudes are for three biological replicates.

\section{PBS excitation}

In cyanobacteria PBSs increase the light absorption and are the major antennas of PSII [34]. The role of PBSs in state transitions is largely ambiguous so far. To selectively excite the PBSs $577 \mathrm{~nm}$ excitation pulses were used for the lifetime imaging of state transitions in S. elongatus cells. The steady-state fluorescence intensity and average fluorescence lifetime of cells in state I (Fig. $4(A, B)$ ) are both higher than those of state II cells (Fig. 4(C,D)), similar to the case of $\mathrm{Chl} a$ excitation.

Upon PBS excitation in a small fraction of cells some regions of high fluorescence are observed, see Fig. 4A (state I) and 4C (state II) "REG 1". Fig. 4B and 4D show that the average lifetime in this region also has a higher value. The fluorescence of PBSs which are connected to the photosystems decays with a lifetime of $120 \pm 20$ ps ${ }^{[26]}$. However, fluorescence decay kinetics of PBSs become slower in case of free and/or partially connected PBSs. Therefore, a higher value of the average lifetime and fluorescence in some regions of a few cells, observed only after PBS excitation is ascribed to free and/or partially connected PBSs. In state II, both fluorescence and average lifetime in "REG 1" are reduced as compared to state I, 
indicating that PSII is also present in this region. In state II another region of high fluorescene and average lifetime is identified in another cell, which is designated as "REG 2".

\section{S. elongatus \\ $577 \mathrm{~nm}$ Excitation}

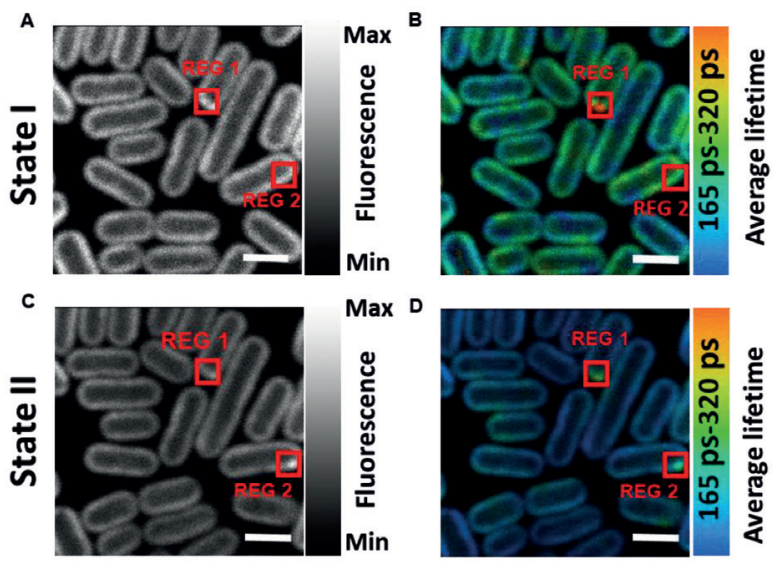

Figure 4. Confocal fluorescence microscope images of $S$. elongatus cells adapted to state I $(A, B)$ and, state II (C,D). A, C show steady-state intensity images of cells in state I and state II, respectively. B, D show average lifetime distributions in state I and state II adapted cells. Same cells were imaged for both state I and State II measurements. Excitation wavelength was $577 \mathrm{~nm}$ and fluorescence was recorded in $685-720 \mathrm{~nm}$ range. Measurements were done at room temperature. "REG 1" in all figures, enclosed in red box shows the area where fluorescence $(A, C)$ and average lifetime $(B, D)$ is higher than the rest of the cell. "REG 2 " shows the area of high fluorescence (C) and average lifetime (D) in state Il cells. "REG 2" in (A,B) is drawn for comparison with "REG 2" in (C,D). All images are equally adjusted for the brightness and contrast. Scale bar is $2 \mu \mathrm{m}$. See supplemental figure S7 for reproducibility of state I and II observed upon selective excitation of PBSs.

\section{S. elongatus \\ 577 nm Excitation}
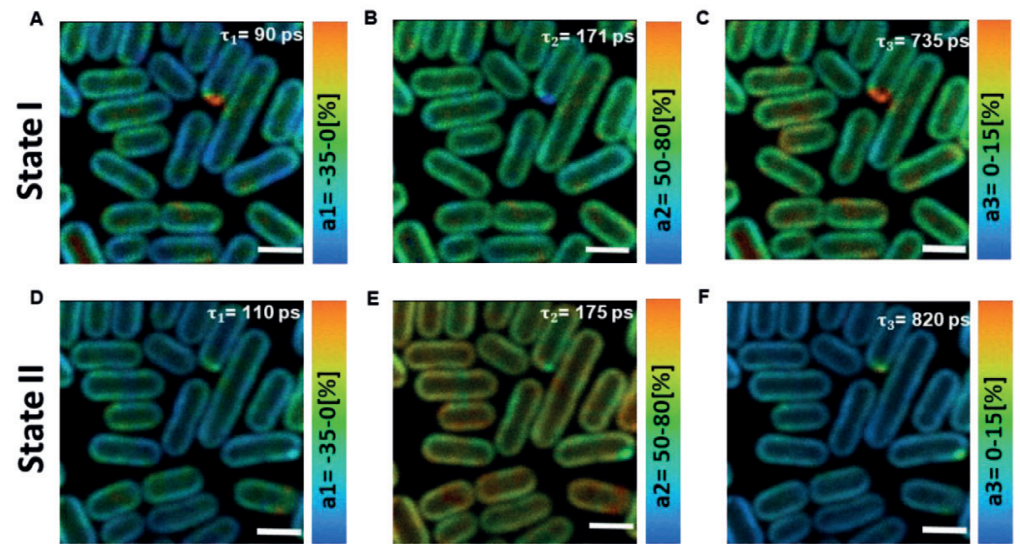

Figure 5. Distribution of amplitudes associated with the fluorescence lifetimes in S. elongatus cells in state I $(A, B, C)$ and state II $(D, E, F)$. Corresponding lifetimes are written in the figures. Images are adjusted for brightness and contrast. Excitation wavelength was $577 \mathrm{~nm}$ and fluorescence was detected in $685 \mathrm{~nm}-720 \mathrm{~nm}$ range. Measurements were performed at room temperature. Scale bar is $2 \mu \mathrm{m}$. 
In the detection range of $685 \mathrm{~nm}-720 \mathrm{~nm}$ three lifetime components $\left(\boldsymbol{\tau}_{1}=\sim 90-110 \mathrm{ps}\right.$, $\boldsymbol{\tau}_{2}=\sim 170-190 \mathrm{ps}$ and $\boldsymbol{\tau}_{3}=\sim 700-1000 \mathrm{ps}$ ) were found sufficient to fit the fluorescence decay across the whole image. These lifetimes are in very good agreement with the ones obtained from the streak-camera measurements on the bulk of S. elongatus cells (Fig. S4). Fig. 5 presents the distribution of the fluorescence amplitudes associated with the obtained lifetime components for cells in state I (Fig.5 (A,B,C)) and state II (Fig. 5(D,E,F)), respectively. The $90-110$ ps component with mostly negative amplitude is very similar for cells in state I (Fig. 5A) and state II (Fig. 5D) and corresponds well with the $\sim 90$ ps component in Fig. S4 streak-camera measurements. This component has been reported to represent excitation energy transfer within PBSs and from PBSs to photosystems ${ }^{[13]}$. The $\sim 170$ ps decay component has the highest amplitude in both states and corresponds with the $\sim 180$ ps component $\left(\lambda_{\max }=683 \mathrm{~nm}\right)$ in streak-camera measurements in Fig. $\mathrm{S} 4$. In state II this component has a higher amplitude (Fig. 5(E)) than in state I (Fig. 5B) across the whole cell and corresponds with the $\sim 180$ ps component in ${ }^{[13]}$. Based on our previous work ${ }^{[13]}$ the $\sim 170$ ps component is ascribed to quenched PSII.
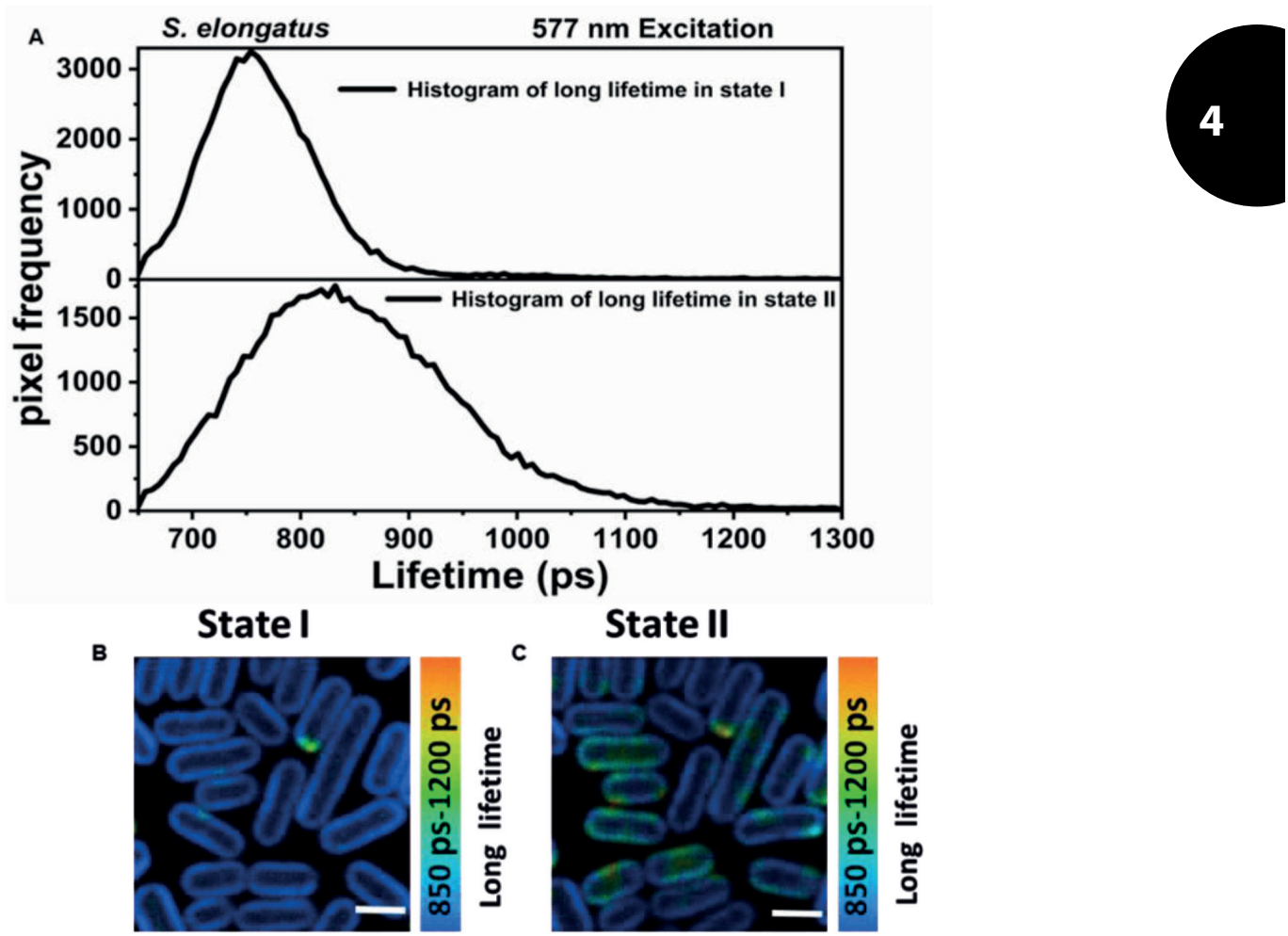

Figure 6. $577 \mathrm{~nm}$ excitation (A) the histogram of long lifetime in state I (upper panel) and state II (lower panel), respectively. Distribution of the long lifetime in range of 850 ps to 1200 ps in state I (B) and state II (C). B and C are corrected for the brightness and contrast. Distribution of the slow lifetime was obtained by fixing the first two lifetimes in the analysis, while the third lifetime was left free. Scale bar in B and C is $2 \mu \mathrm{m}$. 
The third lifetime component ( $\boldsymbol{\tau}_{3}=\sim 735 \mathrm{ps}$ (state I) $820 \mathrm{ps}$ (state II)) in Fig. 5 has considerably higher amplitude in state I as compared to state II and is comparable with the $\sim 0.8-1.0 \mathrm{~ns}$ component $\left(\lambda_{\max }=683 \mathrm{~nm}\right)$ in streak measurements in Fig. $S 4$ representing slow PSII decay and decay from some disconnected PBSs. The lifetime of this component reproducibly had a slightly higher value in state II as compared to state I in our FLIM measurements. This observation is in agreement with the previous time-resolved spectroscopic works on S. elongatus ${ }^{[13]}$ and Synechococcus $6301^{[35]}$. Interestingly, this was not the case upon Chl $a$ excitation.

The histogram of the long lifetime (Fig. 6A) shows that for state II cells, the amount of pixels with longer lifetimes ( 850 ps to 1200 ps) is higher than for state I cells. The distribution of the long lifetime in the range of $850-1200$ ps for cells in state I and state II is shown in Fig. $6 \mathrm{~B}$ and $6 \mathrm{C}$ respectively.

\section{Discussion}

State transitions in cyanobacteria have been extensively studied for many decades, however, no consensus has been reached so far about the exact roles of PSI, PSII and PBSs during the process. In this work we apply FLIM to investigate state transitions at the cellular level in living S. elongatus cells. FLIM was used to visualize the distribution of PSI, PSII and PBSs, based on their autofluorescence. As such the need to introduce fluorescent tags on photosynthetic proteins was avoided and state transitions could be followed under near native conditions. The fluoresence kinetics of cells in state I were compared with the same cells in state II. The FLIM results were also compared with the streak-camera data to better interpret the fluorescence kinetics obtained from the cell ensembles. The main objective of this work was to bring clarity about the roles of PSI, PSII and PBSs by correlating the distribution of fluorescence changes during state transitions with the distribution of photosynthetic pigments in thylakoid membranes.

\section{Distribution of photosystems and state transitions}

Upon selective excitation of the photosystems (440 nm excitation) a $\sim 20$ ps fluorescence component from PSI and two lifetimes components of $\sim 180$ ps and $\sim 750-820$ ps from PSII (Fig. 2) were resolved. FLIM results (Fig. 2) show that heterogeneity of the PSI and PSII distribution in S. elongatus cells occurs in two ways. Firstly, PSI appears to be more abundunt in the inner thylakoid(s) as indicated by the gradual increase in the percentage amplitude of the PSI representative fluorescence signal ( 20 ps component) from periphery towards the inner regions of the five representative cells (Fig. 2 and Fig. S5). The percentage distribution of PSII decreases from outer thylakoid(s) to inner thylakoids. The enrichement of PSI in the inner thylakoid layers has been reported before for Synechococcus PCC $79422^{[24]}$ and Synechocystis $6803^{[23,36]}$. Additionaly, pockets of high PSI (low PSII) content and low PSI (high PSII) content 
(Fig. 3) were found in both states in the thylakoids of individual cells. Despite $\sim 30$ minutes of difference in state I and state II measurements, the location of regions of high/low PSI/PSII ratio was the same in both states. This type of lateral and partial segregation of PSI and PSII, composing a mosaic like strutcure of stable microdomains in thylakoids of Synechocystis sp. PCC 6803 was recently reported in ${ }^{[37]}$. This microscale heterogeneous organization of PSI and PSII in cyanobacterial thylakoids was speculated to be an evoloutionary/functional precurser for the granal/stromal hetrogeneity in photosystems in higher plants ${ }^{[25,37]}$. Clusters enriched in PSI and PSII in thylakoids of S. elongatus were also visualized in ${ }^{[24]}$. In various species of cyanobacteria a lateral segregation of PSI in thylakoid membranes was resolved in [38]. However, a complete separation of PSI and PSII has not been observed in this study or the previous studies ${ }^{[37]}$.

Upon state I to state II transition, a decrease in the amplitude of the slowest component (735-820 ps) was observed with a concomittant increase in the amplitude of the $\sim 180 \mathrm{ps}$ component as compared to state I (Fig. 2), similar to the streak-camera measurements on an ensemble of cells. As a result the average lifetime and fluorescence intensity for state II was reduced compared to cells in state I (Fig. 1). The reduced PSII fluorescence is nearly homogeneous (Fig. 2-3, Fig. S5) in all parts of the cells irrespective of the inhomogeneous distribution of PSI and PSII troughout the cell. This observation show that the amount of PSI does not correlate with the level of state II transition in S. elongatus.

\section{PSII quenching in state I and II}

In this work, upon selective $\mathrm{Chl} a$ excitation a component of 170-190 ps was observed in both states. Recent studies have shown that this component represents fluorescence decay of PSII ${ }^{[13,31]}$. An increase in the amplitude of this component in state II at the expense of the slowest component (Fig. 2) was concluded to show the quenching of PSII in state II ${ }^{[13]}$. However, as this decay lifetime is present in both states, it can be suggested that also in state I the quenching of PSII complexes exists already for part of the complexes. Based on these observations we propose that in S. elongatus a certain quenching process intrinsic to the PSII core exists independent of state I or II. Only the extent of PSII quenching is increased in State II to balance the photochemistry of PSI and PSII. Furthermore, very similar fluorescence kinetics observed at room temperature for state I and state II in $S$. elongatus, Synechocystis $6803^{[13]}$ and, Synechococcus $6301{ }^{[35]}$ suggest that the state transitions process is similar in different cyanobacteria species.

\section{$577 \mathbf{~ n m}$ excitation: fluorescence quenching and disconnection of PBS}

$577 \mathrm{~nm}$ excitation was used to selectively excite the PBSs in FLIM measurements and fluorescence was detected between $685 \mathrm{~nm}$-and $720 \mathrm{~nm}$. Based on the fluorescence kinetics the distribution of PBSs and PSII was imaged across the cells. As was found for Chl $a$ excitation, a significant decrease in the average lifetime and fluorescence intensity was 
observed in state II as compared to state I (Fig. 4), which is ascribed to PSII quenching in state II in agreement with earlier results ${ }^{[13]}$.

The FLIM results show that in some cells, there are regions where the average lifetime is significantly longer than in the rest of the cell. We ascribe this to the contribution of disconnected PBSs which have a lifetime of 1.4-1.6 ns ${ }^{[39]}$. Fig. 4B shows one such region "REG 1" in state I cells. In state II an additional region, designated as "REG 2" is identified (Fig. 4D). At least two clearly identifiable regions of disconnected PBSs in state II (Fig. 4D) as compared to only one prominent region in state I (Fig. 4B) show that, the disconnection of the PBSs can occur during the time of the experiment. It is important to note that with FLIM not only the presence, but also the location of disconnected PBSs can be resolved, which is not the case for ensemble measurements. However, it should be noted that such type of disconnection of PBSs was only observed in some of the replicates.

After $577 \mathrm{~nm}$ excitation the longest lifetime in state II cells represents the average of unquenched PSII and slowly decaying disconnected/poorly connected PBSs. The comparison of the distribution of the longest lifetime component in the 850 ps -1200 ps range in state I (Fig. 6B) and state II (Fig. 6C) indicates that regions of disconnected/ poorly connected PBSs are present in a majority of state II cells. Fluorescence from such regions in state II slightly offsets the fluorescence decrease observed at $683 \mathrm{~nm}$ in state II, due to the quenching of PSII. PBSs are water soluble proteins and their mobility along the thylakoid membranes of state transitions performing cyanobacterium S. elongatus ${ }^{[40]}$ and mesophilic red algae $P$. Cruentum ${ }^{[41]}$ has been shown with FRAP microscopy. A slow decoupling of PBSs in Synechocystis sp. PCC6803 after a long-term exposure ( $>1 \mathrm{~h}$ ) to high light was observed with confocal microscopy ${ }^{[42]}$. High intensities of light absorbed either by PBSs or photosystems, induce an excitonic decoupling of PBSs followed by their disassembly and detachment from thylakoid membrane ${ }^{[43]}$. This type of uncoupling of PBSs occurs at the timescale of a few seconds and the physiological purpose is the photoprotection of reaction-centre proteins ${ }^{[43]}$. A very small fraction of uncoupled PBSs resolved in this work and in ${ }^{[26]}$ at very low excitation powers does not appear to have any physiological significance for photoprotection or state transitions. It can however not completely be ruled out that the laser power used to close the reaction centres also induces some uncoupling of a very small amount of PBSs. The higher value of the longest lifetime in state II is most probably caused by the presence of a fraction of disconnected PBSs in both states. In state II the relative contribution of PSII to the longest lifetime is smaller and therefore the average value of the longest lifetime will be influenced more by the long lifetime of the disconnected PBSs 


\section{PBSs and PSII quenching in state II}

In this work we have shown that increased PSII quenching in state II as compared to state I occurs for state transitions in cyanobacteria and is responsible for the commonly observed change in the ratio of PSII/PSI fluorescence. As PBSs do not play a role in redistributing the energy between PSI and PSII ${ }^{[12]}$, it is tempting to conclude they are not involved at all in state transitions. However, upon selective excitation of PSs, the $\triangle A p C D$ mutant strains of Synechocystis $6803^{[13,22]}$ and, Synechococcus 7002 [10] show reduced PSIl quenching in state II as compared to their WT counterparts. The ApcD component is situated at the bottom of the PBS core and is in direct interaction with the PS complexes ${ }^{[44]}$. It thus appears that the interaction between PBS and PS plays a role in state transitions, possibly by regulating the reorganisation of PSII complexes. The exact mechanism by which PBSs are involved in state transitions remains to be investigated.

\section{Conclusions}

This study shows that in S. elongatus two (sub)populations of PSII, namely quenched and unquenched, exist in state I and state II. The equilibrium between quenched and unquenched PSII is changed upon state transitions. The PSII fluorescence quenching in state II was found to be nearly homogeneous throughout the cells. The exact mechanism of PSIl quenching in state II is yet to be determined. However, taken into consideration the observations from previous studies, it may be that the interaction between PBS and PSII needs to allow for a reorganisation of PSII complexes in state II which are required for PSII quenching. 


\section{References}

1. Mullineaux, C.W. and J.F. Allen, State 1-State 2 transitions in the cyanobacterium Synechococcus 6301 are controlled by the redox state of electron carriers between Photosystems I and II. Photosynthesis Research, 1990. 23(3): p. 297-311.

2. Mullineaux, C.W., Electron transport and light-harvesting switches in cyanobacteria. Frontiers in plant science, 2014. 5: p. 7-7.

3. Allen, J.F., et al., Chloroplast protein phosphorylation couples plastoquinone redox state to distribution of excitation energy between photosystems. Nature, 1981. 291 (5810): p. 25-29.

4. Rochaix, J.-D., Regulation and Dynamics of the Light-Harvesting System. Annual Review of Plant Biology, 2014. 65(1): p. 287-309.

5. Staehelin, L.A. and C.J. Arntzen, Regulation of chloroplast membrane function: protein phosphorylation changes the spatial organization of membrane components. The Journal of cell biology, 1983. 97(5 Pt 1): p. 1327-1337.

6. Wientjes, E., H. van Amerongen, and R. Croce, LHCII is an antenna of both photosystems after long-term acclimation. Biochimica et Biophysica Acta (BBA) - Bioenergetics, 2013. 1827(3): p. 420-426.

7. Murata, N., Control of excitation transfer in photosynthesis I. Light-induced change of chlorophyll a fluoresence in Porphyridium cruentum. Biochimica et Biophysica Acta (BBA) - Bioenergetics, 1969. 172(2): p. 242-251.

8. Kirilovsky, D., Modulating energy arriving at photochemical reaction centers: orange carotenoid protein-related photoprotection and state transitions. Photosynthesis research, 2015. 126(1): $\mathrm{p}$. 3-17.

9. Calzadilla,P.I.,etal.,The Cytochrome\&lt;em\&gt;b\&lt;/em\&gt;\&lt;sub\&gt;6\&/t;/sub\&gt;\&lt;em\&gt;\&/t;/ em\&gt; Complex Is Not Involved in Cyanobacterial State Transitions. The Plant Cell, 2019. 31(4): p. 911.

10. McConnell, M.D., et al., Regulation of the distribution of chlorophyll and phycobilin-absorbed excitation energy in cyanobacteria. A structure-based model for the light state transition. Plant physiology, 2002. 130(3): p. 1201-1212.

11. Chukhutsina, V., et al., Cyanobacterial Light-Harvesting Phycobilisomes Uncouple From Photosystem I During Dark-To-Light Transitions. Scientific reports, 2015. 5: p. 14193-14193.

12. Ranjbar Choubeh, R., et al., State transitions in the cyanobacterium Synechococcus elongatus 7942 involve reversible quenching of the photosystem II core. Biochimica et Biophysica Acta (BBA) - Bioenergetics, 2018. 1859(10): p. 1059-1066.

13. Bhatti, A.F., et al., State transitions in cyanobacteria studied with picosecond fluorescence at room temperature. Biochimica et Biophysica Acta (BBA) - Bioenergetics, 2020: p. 148255.

14. Mullineaux, C.W., M.J. Tobin, and G.R. Jones, Mobility of photosynthetic complexes in thylakoid membranes. Nature, 1997. 390(6658): p. 421-424. 
15. Allen, J.F., C.E. Sanders, and N.G. Holmes, Correlation of membrane protein phosphorylation with excitation energy distribution in the cyanobacterium Synechococcus 6301. FEBS Letters, 1985. 193(2): p. 271-275.

16. Bruce, D., et al., Mechanism of the light state transition in photosynthesis. IV. Picosecond fluorescence spectroscopy of Anacystis nidulans and Porphyridium cruentum in state 1 and state 2 at 77 K. Biochimica et Biophysica Acta (BBA) - Bioenergetics, 1985. 806(2): p. 237-246.

17. Olive, J., et al., Ultrastructure and light adaptation of phycobilisome mutants of Synechocystis PCC 6803. Biochimica et Biophysica Acta (BBA) - Bioenergetics, 1997. 1319(2): p. 275-282.

18. Bos, P., et al., Digitonin-sensitive LHCll enlarges the antenna of Photosystem I in stroma lamellae of Arabidopsis thaliana after far-red and blue-light treatment. Biochimica et Biophysica Acta (BBA) - Bioenergetics, 2019. 1860(8): p. 651-658.

19. Dong, C. and J. Zhao, ApCD is required for state transition but not involved in blue-light induced quenching in the cyanobacterium Anabaena sp. PCC7120. Chinese Science Bulletin, 2008. 53(21): p. 3422-3424.

20. Dong, C., et al., ApcD is necessary for efficient energy transfer from phycobilisomes to photosystem I and helps to prevent photoinhibition in the cyanobacterium Synechococcus sp. PCC 7002. Biochimica et Biophysica Acta (BBA) - Bioenergetics, 2009. 1787(9): p. 1122-1128.

21. Ashby, M.K. and C.W.Mullineaux, The role of ApcD and ApcFinenergy transfer from phycobilisomes to PS I and PS II in a cyanobacterium. Photosynthesis Research, 1999. 61(2): p. 169-179.

22. Calzadilla, P.I., et al., Different roles for ApcD and ApcF in Synechococcus elongatus and Synechocystis sp. PCC 6803 phycobilisomes. Biochimica et Biophysica Acta (BBA) - Bioenergetics, 2019. 1860.

23. Vermaas, W.F.J., et al., \&lt;em\&gt;In vivo\&/t;/em\&gt; hyperspectral confocal fluorescence imaging to determine pigment localization and distribution in cyanobacterial cells. Proceedings of the National Academy of Sciences, 2008. 105(10): p. 4050.

24. Casella, S., et al., Dissecting the Native Architecture and Dynamics of Cyanobacterial Photosynthetic Machinery. Molecular plant, 2017. 10(11): p. 1434-1448.

25. Konert, G., et al., Protein arrangement factor: a new photosynthetic parameter characterizing the organization of thylakoid membrane proteins. Physiologia Plantarum, 2019. 166(1): p. 264-277.

26. Krumova, S.B., et al., Monitoring photosynthesis in individual cells of Synechocystis sp. PCC 6803 on a picosecond timescale. Biophysical journal, 2010. 99(6): p. 2006-2015.

27. Moronta-Barrios, F., J. Espinosa, and A. Contreras, Negative control of cell size in the cyanobacterium Synechococcus elongatus PCC 7942 by the essential response regulator RpaB. FEBS Letters, 2013. 587(5): p. 504-509.

28. Nevo, R., et al., Thylakoid membrane perforations and connectivity enable intracellular traffic in cyanobacteria. The EMBO journal, 2007. 26(5): p. 1467-1473.

29. Nozue, S., et al., Characterization of thylakoid membrane in a heterocystous cyanobacterium and green alga with dual-detector fluorescence lifetime imaging microscopy with a systematic change of incident laser power. Biochimica et Biophysica Acta (BBA) - Bioenergetics, 2016. 1857(1): p. 46-59. 
30. van Oort, B., et al., Picosecond fluorescence of intact and dissolved PSI-LHCl crystals. Biophysical journal, 2008. 95(12): p. 5851-5861.

31. Tian, L., S. Farooq, and H. Van Amerongen, Probing the picosecond kinetics of the photosystem II core complex in vivo. Physical chemistry chemical physics : PCCP, 2013. 15.

32. Gobets, B., et al., Time-Resolved Fluorescence Emission Measurements of Photosystem I Particles of Various Cyanobacteria: A Unified Compartmental Model. Biophysical Journal, 2001. 81(1): p. 407-424.

33. Mahbub, M., et al., mRNA localization, reaction centre biogenesis and thylakoid membrane targeting in cyanobacteria. Nature Plants, 2020. 6(9): p. 1179-1191.

34. Watanabe, M., et al., Attachment of phycobilisomes in an antenna-photosystem I supercomplex of cyanobacteria. Proceedings of the National Academy of Sciences of the United States of America, 2014. 111(7): p. 2512-2517.

35. Mullineaux, C.W., et al., Picosecond time-resolved fluorescenceemission spectra indicate decreased energy transfer from the phycobilisome to Photosystem II in light-state 2 in the cyanobacterium Synechococcus 6301. Biochimica et Biophysica Acta (BBA) - Bioenergetics, 1990. 1015(2): p. 231-242.

36. Collins, A.M., et al., Photosynthetic pigment localization and thylakoid membrane morphology are altered in Synechocystis 6803 phycobilisome mutants. Plant physiology, 2012. 158(4): p. 1600-1609.

37. Strašková, A., et al., Pigment-protein complexes are organized into stable microdomains in cyanobacterial thylakoids. Biochimica et Biophysica Acta (BBA) - Bioenergetics, 2019. 1860(12): p. 148053.

38. MacGregor-Chatwin, C., et al., Lateral Segregation of Photosystem I in Cyanobacterial Thylakoids. The Plant Cell, 2017. 29(5): p. 1119.

39. Tian, L., et al., Picosecond kinetics of light harvesting and photoprotective quenching in wildtype and mutant phycobilisomes isolated from the cyanobacterium Synechocystis PCC 6803. Biophysical journal, 2012. 102(7): p. 1692-1700.

40. Joshua, S. and C.W. Mullineaux, Phycobilisome Diffusion Is Required for Light-State Transitions in Cyanobacteria. Plant Physiology, 2004. 135(4): p. 2112.

41. Kaňa, R., et al., Phycobilisome Mobility and Its Role in the Regulation of Light Harvesting in Red Algae. Plant physiology, 2014. 165(4): p. 1618-1631.

42. Steinbach, G. and R. Kaňa, Automated Microscopy: Macro Language Controlling a Confocal Microscope and its External Illumination: Adaptation for Photosynthetic Organisms. Microscopy and Microanalysis, 2016. 22: p. 258-263.

43. Tamary, E., et al., Structural and functional alterations of cyanobacterial phycobilisomes induced by high-light stress. Biochimica et Biophysica Acta (BBA) - Bioenergetics, 2012. 1817(2): p. 319327.

44. Zhang, J., et al., Structure of phycobilisome from the red alga Griffithsia pacifica. Nature, 2017. 551. 


\section{Supplementary Information}

\section{$440 \mathrm{~nm}$ Excitation}
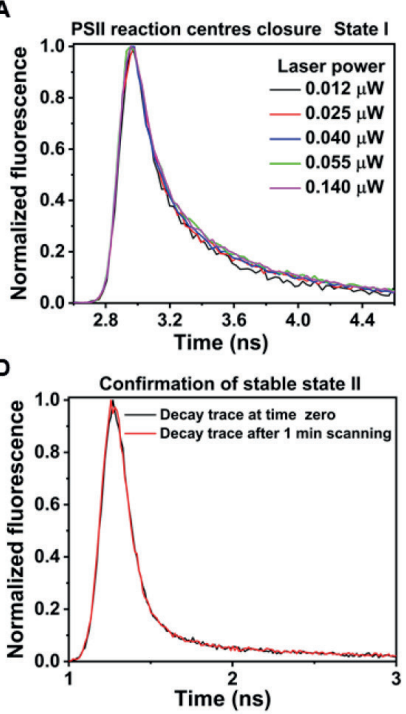

B

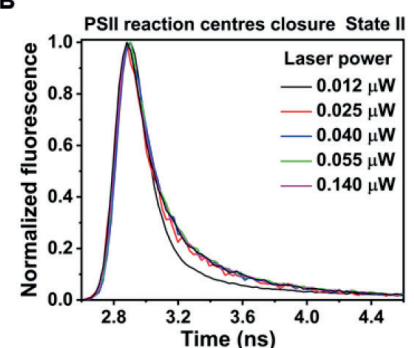

E

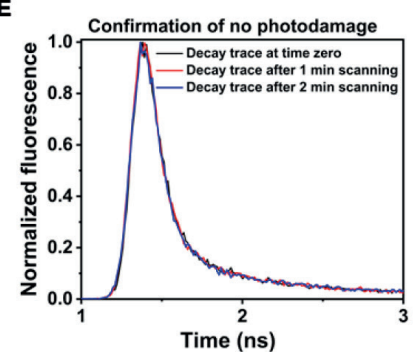

C

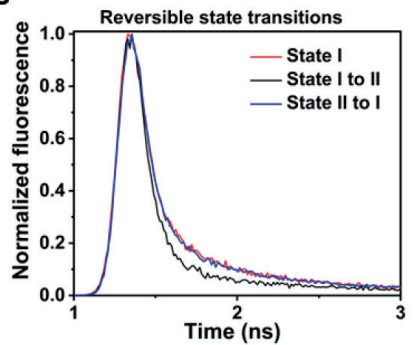

Figure. S1. Fluorescence kinetics at $440 \mathrm{~nm}$ laser scanning. Fluorescence kinetics as a function of power of excitation pulses for cells in state I (A) and state II (B). (C) Decay traces from cells equilibrated in state I (red solid line), cells brought from state I to state II (black line) and cells brought back to state I (blue line). (D) Black line shows a decay trace recorded for $1 \mathrm{sec}$ after fully adapting the cells in state II and is indicated as time zero trace. Red line shows the decay trace at the end of 1 minute of laser scanning. (E) Black, red and, blue lines show the decay traces at the beginning, after 1 minute and after 2 min of laser scanning of state I adapted cells. $440 \mathrm{~nm}$ excitation pulses were used and fluorescence was detected at $685-720 \mathrm{~nm}$. All measurements were performed at room temperature. Cells were adapted for 30 minutes to blue light to induce state I and were adapted to darkness for the same time to induce state II.

In Fig. S1A fluorescence kinetics for excitation powers of $\sim 0.01 \mu \mathrm{W}$ to $0.14 \mu \mathrm{W}$ (440 nm light) are relatively similar. For state II cells in Fig. S1B the PSII fluorescence kinetics is much faster at $\sim 0.01 \mu \mathrm{W}$ power as compared to the kinetics obtained after $0.025 \mu \mathrm{W}$ of laser power excitation. However, the fluorescence kinetics did not slow down further when the power was increased even up to $0.14 \mu \mathrm{W}$. It shows that the PSII reaction centers were closed at all powers except at the lowest one $(0.1 \mu \mathrm{W})$. In comparison in state I (Fig. S1A) PSIl reaction centers are predominantly closed for all laser powers tested. All further measurements at $440 \mathrm{~nm}$ excitation were performed with $0.025 \mu \mathrm{W}$ laser power. Fig. S1C shows the fluorescence decay traces from cells which were first adapted to state I (red line) and then state II (black line) and finally back to state I (blue line). Decay traces which are shown in red and blue are very similar, reflecting that the state transitions were reversible in our samples. We used $440 \mathrm{~nm}$ pulses to selectively excite the photosystems (Chl $a$ ); however the light of similar wavelengths (blue light) is used to bring the cells in state I. In order to ensure that cells adapted to state II remain in state II when $440 \mathrm{~nm}$ excitation light 
is used, a decay trace at the beginning of the laser scanning (black line in Fig. S1D) was compared with the trace after 1 min of scanning (red line in Fig. S1D). Both traces are very similar, showing that cells were stable in state II for the laser scanning parameters used in this work. Both decay traces were measured for $1 \mathrm{sec}$. In our measurements, the same cells were scanned for a total time of $\sim 2$ minutes ( $1 \mathrm{~min}$ for state I plus $1 \mathrm{~min}$ for state II). Fig. S1E shows the decay traces measured of light-adapted cells at the beginning of the scanning (black line), after 1 min of scanning (red line) and after 2 min of scanning (blue line). All these traces were found to be almost identical, showing that there was no detectable photodamage in the cells during the measurements.

\section{7 nm Excitation}

A
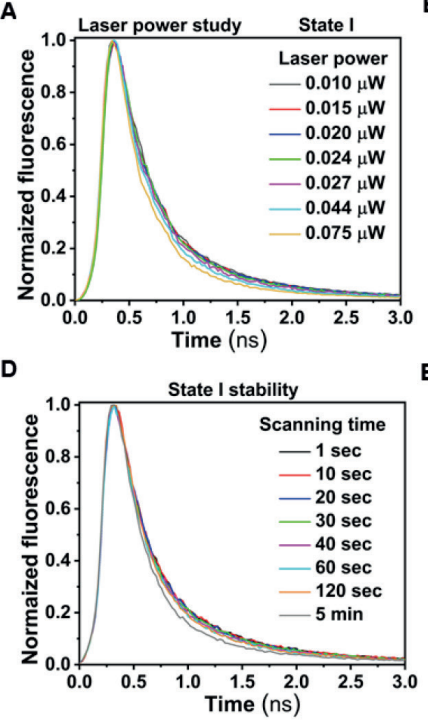

B

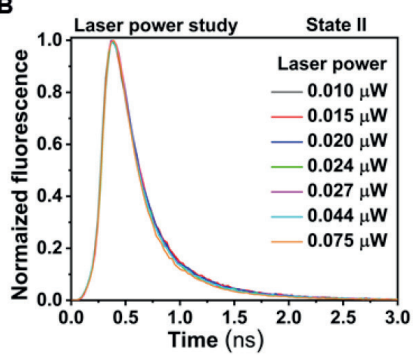

C

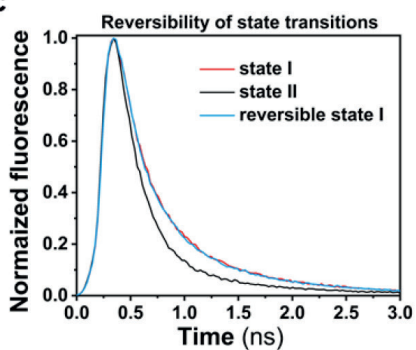

E

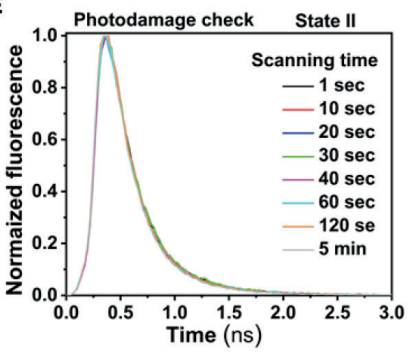

Figure. S2. Fluorescence kinetics at $577 \mathrm{~nm}$ laser scanning. Fluorescence decay traces of S. elongatus cells as a function of power of excitation pulses for cells in state I (A) and state II (B). (C) Decay traces from cells equilibrated in state I at various times of laser scanning. (D) Decay traces from cells equilibrated in state II at various times of laser scanning. (E) Decay traces from cells equilibrated in state I (red solid line), cells brought from state I to state II (black line). Cells brought back to state I (blue line). $577 \mathrm{~nm}$ excitation pulses were used and fluorescence was detected at $685-720 \mathrm{~nm}$. All measurements were performed at room temperature. Cells were adapted for 30 minutes to blue light to induce state I and were adapted to darkness for the same time to induce state II.

In Fig. S2A fluorescence kinetics for excitation powers of $0.010 \mu \mathrm{W}$ to $0.020 \mu \mathrm{W}$ are very similar for state I cells. At $0.024 \mu \mathrm{W}$ fluorescence decay started to become slightly faster (green line) and was significantly faster at laser powers of $0.044 \mu \mathrm{W}$ (cyan line) and 0.075 $\mu \mathrm{W}$ (orange line). In Fig. S2B fluorescence kinetics were very similar from $0.010 \mu \mathrm{W}$ to 0.027 $\mu \mathrm{W}$ and became a little faster at excitation powers of $0.044 \mu \mathrm{W}$ and $0.075 \mu \mathrm{W}$. In both cases (Fig. S1A and S1B) fluorescence kinetics did not slow down when excitation power was increased from $0.01 \mu \mathrm{W}$ showing that PSII reaction centres were closed at all powers 
tested. All further measurements at $577 \mathrm{~nm}$ were performed at $0.01 \mu \mathrm{W}$ laser power. Fig. S1C shows the fluorescence decay traces from cells which were first adapted to state I (red line) and then to state II (black line) and finally back to state I (blue line). Decay traces which are shown in red and blue are very similar, reflecting that the state transitions were reversible in our samples. For the preferential excitation of PBSs, $577 \mathrm{~nm}$ pulses were used; however the light of similar wavelengths (yellow-orange) can bring the cells in state II. To ensure that, cells adapted to state I remained in state I when $577 \mathrm{~nm}$ excitation was used, decay traces at various times of laser scanning were compared. Fig. S1D shows that decay traces measured at the beginning of laser scanning (black line) until 30 seconds of scanning (green line) are very similar. After 40 seconds of laser scanning fluorescence decay became noticeably faster (magenta line) and a significant fastening in fluorescence decay was observed only after 5 minutes of scanning (grey line). All further measurements at $577 \mathrm{~nm}$ excitation were done with 20 seconds of laser scanning. Fig. S1E shows that in state II cells, fluorescence decay traces at the beginning of laser scanning (black line) until 5 minutes of scanning (grey line) are very comparable, showing that laser power did not result in detectable photodamage to the samples.

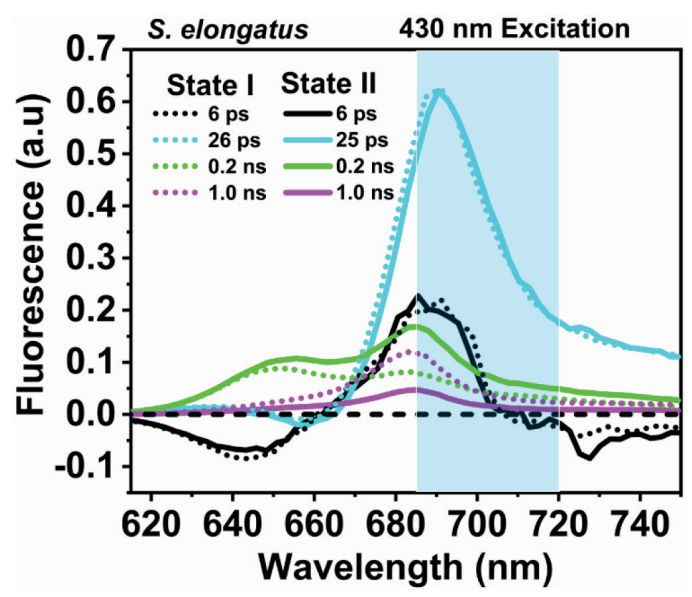

Figure. S3. Decay-associated spectra (DAS) obtained from global fitting of time-resolved fluorescence results upon $430 \mathrm{~nm}$ excitation of S. elongatus cells in state I (dotted lines) and state II (solid lines). The slowest lifetime in each case was fixed to $1.0 \mathrm{~ns}$. The 20 ps DAS in each case is multiplied with 0.5 to make the two slowest DAS better visible. DAS-associated lifetimes are shown in the inset with corresponding colours. The blueish rectangle shows the fluorescence detection region for FLIM measurements in this work. The figure is reproduced from our previous work (Bhatti et al., 2020), published under CC-BY-4.0 licence. 


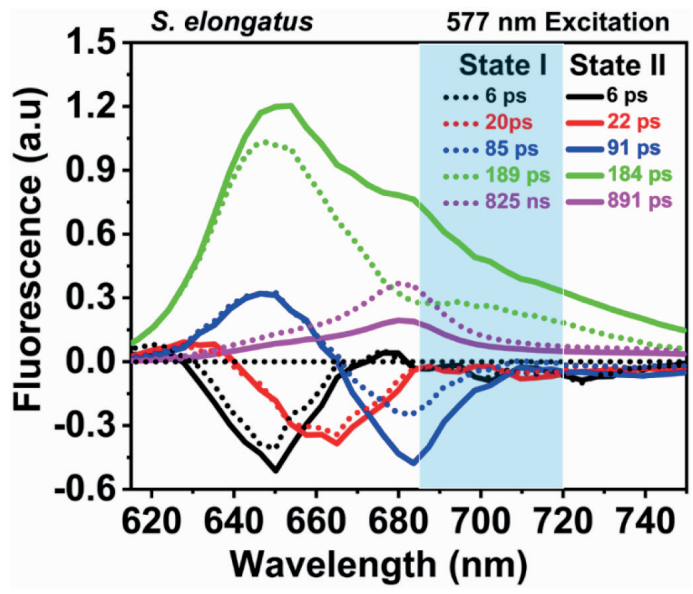

Figure. S4. Decay-associated spectra obtained from global fitting of time-resolved fluorescence results after $577 \mathrm{~nm}$ excitation of S. elongatus cells in state I (dotted lines) and state II (solid lines). DAS-associated lifetimes are presented in the inset with corresponding colours. The bluish rectangle shows the fluorescence detection region for FLIM measurements in this work. The figure is reproduced from our previous work (Bhatti et al., 2020), published under CC-BY-4.0 licence.
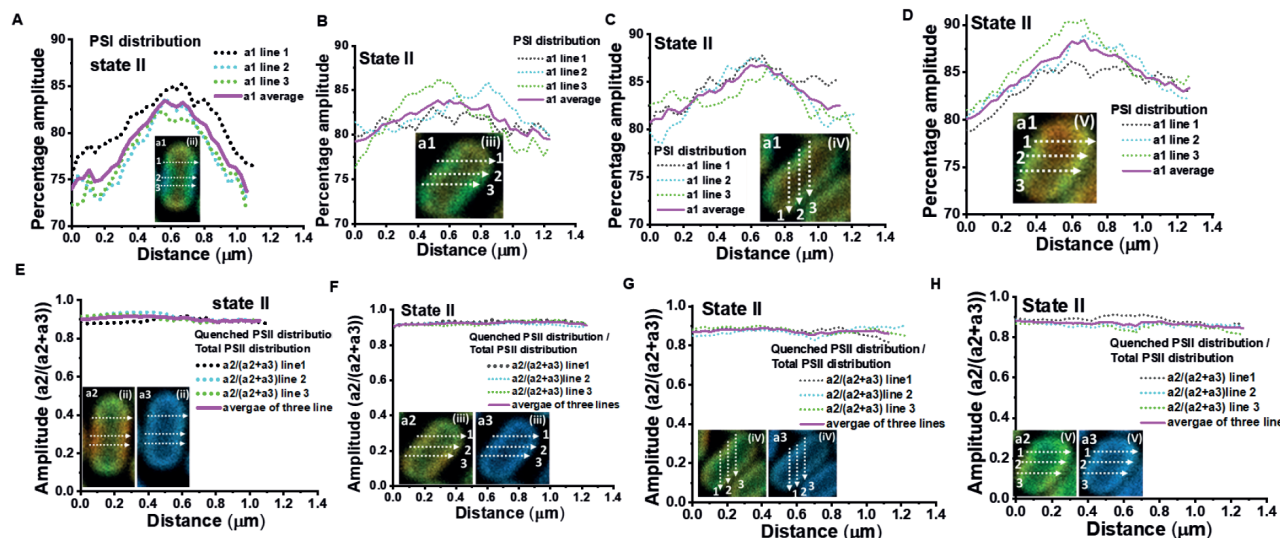

Figure. S5. (A,B,C,D) Profile of PSI percentage acquired across the width of the cells "ii-v" (marked with white rectangles in Fig. 2) along three lines (dotted black, dotted green and dotted cyan). Average of 3 lines is shown with solid magenta line. (E,F,G,H) Ratio of quenched PSII/total PSII (a2/(a2+a3) acquired across the width of the cells "ii-v" (marked with white rectangles in Fig. 2) along three lines (dotted black, dotted green and dotted cyan). Average of thrree lines is shown with the solid magenta line. Cells "ii- $v$ " are shown in inset. 


\section{S. elongatus}

\section{$440 \mathrm{~nm}$ Excitation}
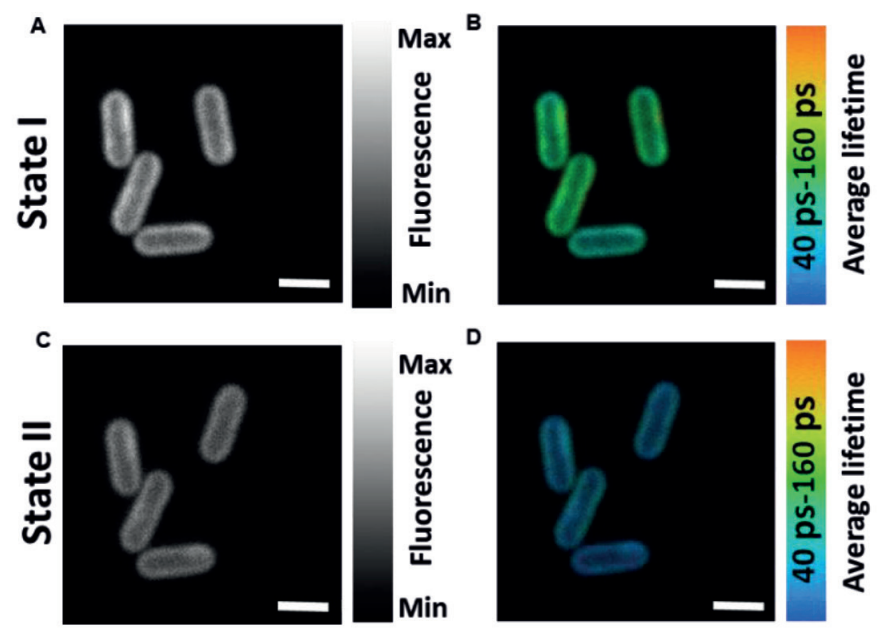

Fig. S6. Confocal fluorescence microscope images of $S$. elongatus cells adapted to state I (A,B) and state II (C,D). A, C show steady-state intensity images of cells in state I and state II, respectively. (C,D) show average lifetime distributions in state-I and state-II adapted cells. Same cells were imaged for both state I and State II measurements. Excitation wavelength was $440 \mathrm{~nm}$ and fluorescence was recorded in $685-720 \mathrm{~nm}$ range. Scale bar is $2 \mu \mathrm{m}$. Images are presented without adjustments for brightness and contrast. Measurements were performed at room temperature.

\section{S. elongatus}

\section{7 nm Excitation}

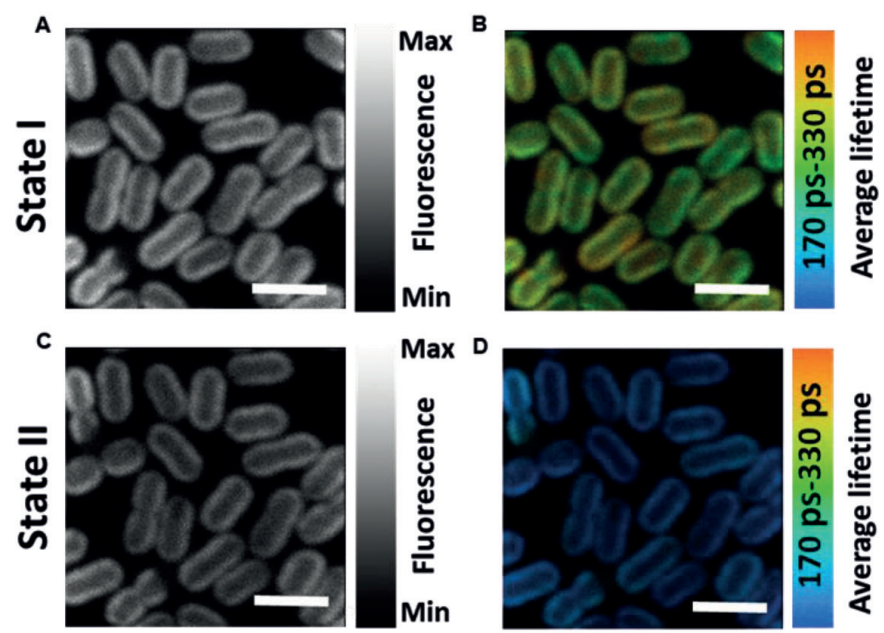

Fig. S7. Confocal fluorescence microscope images of $S$. elongatus cells adapted to state I $(A, B)$ and state II $(C, D)$. A, C show steady-state intensity images of cells in state I and state II, respectively. (C,D) show average lifetime distributions in state-I and state-II adapted cells. Same cells were imaged for both state I and State II measurements. Excitation wavelength was $577 \mathrm{~nm}$ and fluorescence was recorded in $685-720 \mathrm{~nm}$ range. Scale bar is $4 \mu \mathrm{m}$. Images are presented without adjustments for brightness and contrast. Measurements were performed at room temperature. 


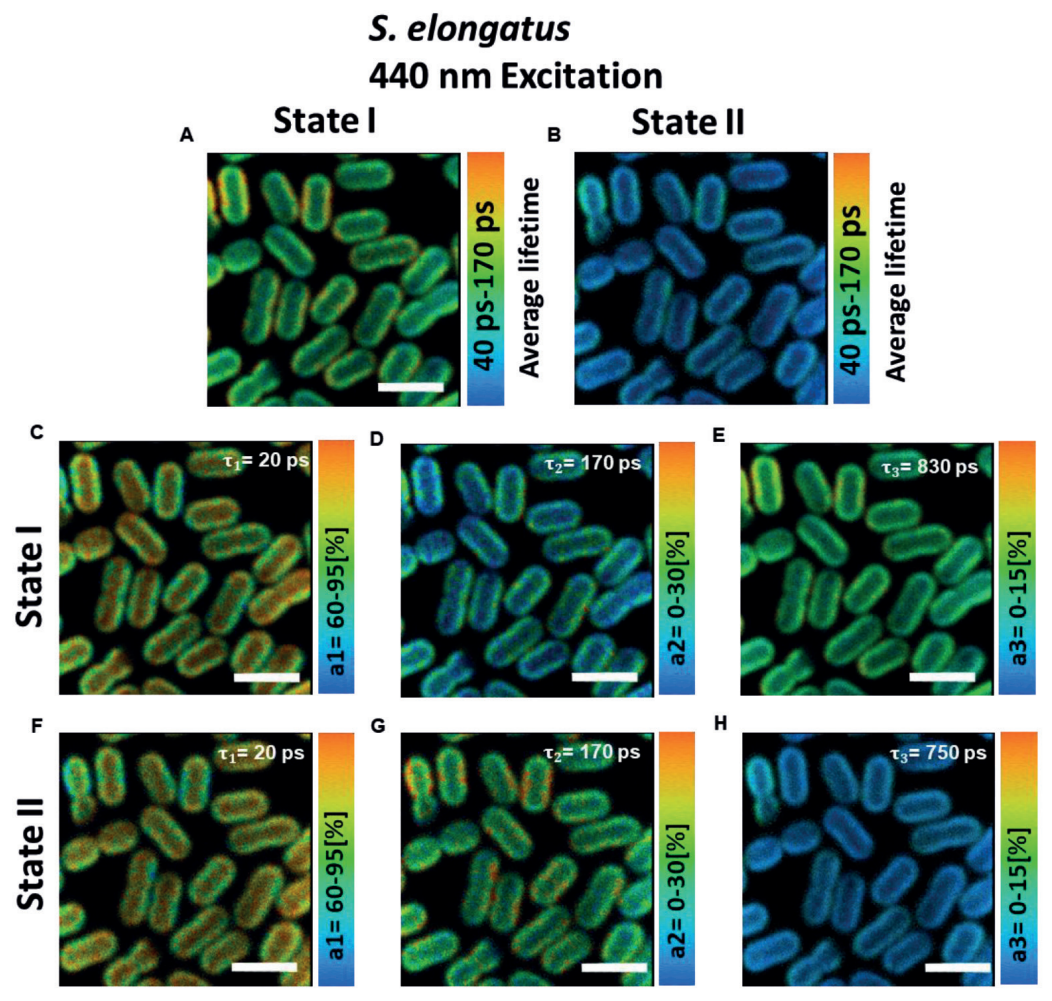

Figure. S8. (A,B) Average fluorescence lifetime distributions in state-I and state-II adapted cells. Distribution of amplitudes associated with the fluorescence lifetimes in $S$. elongatus cells in state I (C,D,E) and state II $(\mathrm{F}, \mathrm{G}, \mathrm{H})$. Corresponding lifetimes are written in the figures. Same cells were imaged for both state I and State II measurements. Excitation wavelength was $440 \mathrm{~nm}$ and fluorescence was recorded in $685-720 \mathrm{~nm}$ range. Scale bar is $4 \mu \mathrm{m}$. Images are adjusted for brightness and contrast. Measurements were performed at room temperature.

In Fig. 1 and Fig. 2, fluorescence lifetime analysis was performed at pixel binning of 7 to achieve better signal/noise ratio for the estimation of fit parameters (lifetimes and amplitudes). The lifetimes found with this analysis were fixed and pixel binning was reduced to 2. Fig. $S 8$ shows the average fluorescence lifetime images $(A, B)$ and amplitude profiles associated with the lifetimes $(\mathrm{C}-\mathrm{H})$ at the reduced pixel binning of 2. Comparison of Fig. $\mathrm{S} 8$ with the Fig. 1 and Fig. 2 shows that the results obtained at lower and higher binning are very similar. At binning 2 , slightly broader distribution of amplitudes was observed as compared to binning 7. A high amplitude of $\sim 20$ ps component (representing PSI) in the centre of the cells can therefore not be ascribed as an artefact of pixel averaging at higher binning. 
CHAPTER

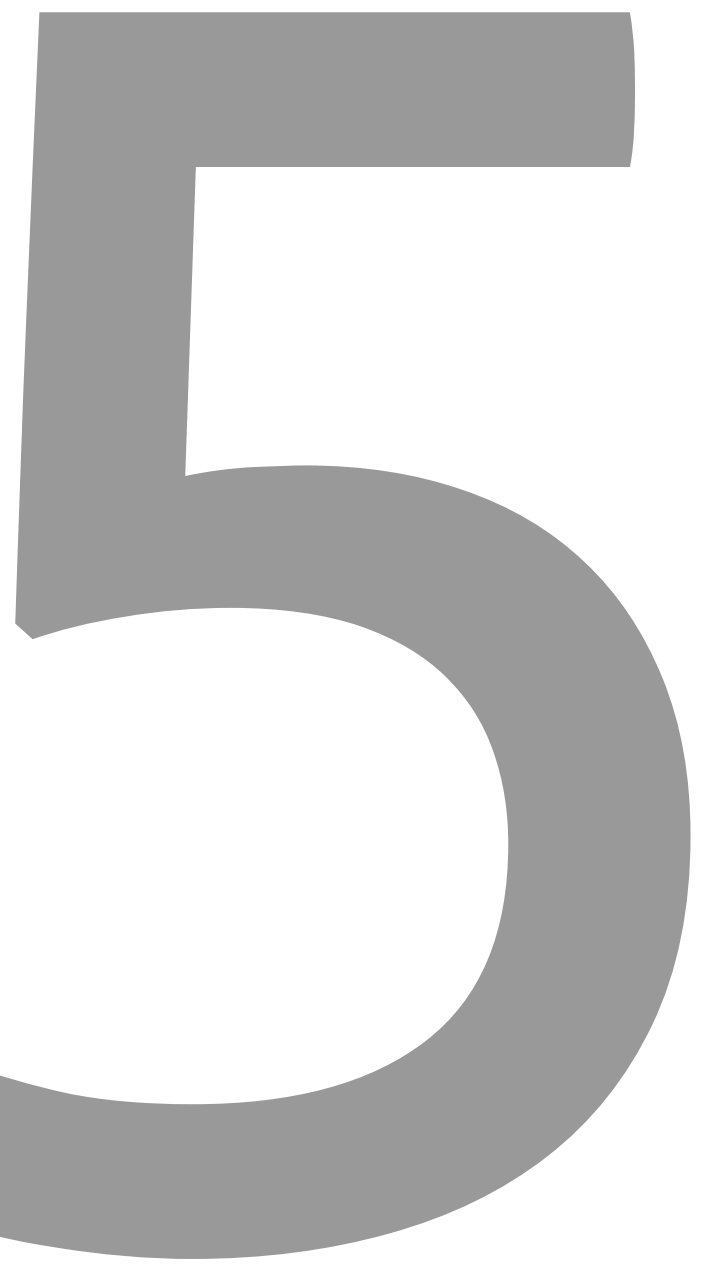




\title{
Far-red allophycocyanin increases both the absorption cross-section and photochemical efficiency of chlorophyll f-containing photosystem II
}

\author{
Vincenzo Mascoli, Ahmad Farhan Bhatti, Luca Bersanini, \\ Herbert van Amerongen, and Roberta Croce
}





\section{Introduction}

Cyanobacteria are oxygenic photoautotrophs that convert light energy into chemical energy and play an essential role as primary producers in various ecosystems. In cyanobacteria, the light-dependent reactions are carried out by the membraneembedded chlorophyll-binding photosystems I and II (PSI and PSII) and by the watersoluble phycobilisomes (PBS). The latter are relatively large complexes (typically several $\mathrm{MDa}$ ) of brilliantly-colored phycobiliproteins (PBPs) and associated linker proteins ${ }^{[1-4]}$. PBPs usually contain covalently-attached pigments named phycobilins. The PBSs, which can bind several hundred pigments, are formidable antennae that harvest photons and deliver their energy to the reaction centers $(R C s)$ of the photosystems ${ }^{[5-7]}$, where charge separation occurs. They therefore increase the absorption cross-section of the photosystems and expand the photosynthetic active spectrum to regions (commonly between 500 and $650 \mathrm{~nm}$ ) where the absorption by the chlorophylls (Chls) is weak. Cyanobacterial PBSs have different architectures, the most common being hemidiscoidal ${ }^{[8,9]}$. Hemidiscoidal PBSs consist of a core composed of two, three, or five cylinders, each containing four discs of trimeric allophycocyanin (APC) subunits ${ }^{[2,10,11]}$. Two of these cylinders, referred to as basal cylinders, are in contact with the membrane and contain the lowest energy bilins, which transfer energy to the Chls of PSI or PSII $[6,7,9,12,13]$. The APC core is connected to several rods of phycocyanin (PC) and, sometimes, phycoerythrin (PE). The downhill energy gradient between the peripheral rods and the APC core enhances the directionality of energy transfer towards the Chl-reach units ${ }^{[14]}$.

Oxygenic photosynthesis relies specifically on $\mathrm{Chl} a$ and is powered by visible light $(400-700 \mathrm{~nm})^{[15]}$. However, some cyanobacteria growing in deep-shaded environments that are highly enriched in far-red light (FRL, 700-800 nm) can harvest less energetic photons using the red-shifted Chls $f$ and $d^{[16-18]}$. While under visible light (such as white light, WL) these strains only produce $\mathrm{Chl} a$ like "conventional" cyanobacteria, under FRL they remodel their photosynthetic apparatus and synthesize $\mathrm{Chl} d$ and $\mathrm{Chl} f$ through an acclimation process called FRL-induced photoacclimation (FaRLiP) ${ }^{[17,19]}$. FaRLiP relies on the expression of a highly conserved 21-gene cluster encoding protein subunits of PSI, PSII, and PBPs, together with the enzyme $\mathrm{Chl} f$ synthase ${ }^{[20]}$. The photosystems assembled in FRL (hereafter referred as FRL-photosystems), which are paralogues of those expressed in WL (WL-photosystems), incorporate the red-shifted Chls in relatively low amounts, while $\mathrm{Chl} a$ remains the most abundant pigment (about $90 \%$ of total $\mathrm{Chls}$ ) ${ }^{[16,21-23]}$. FRL-PSII binds up to $4 \mathrm{Chls} f$ and $1 \mathrm{Chl} d$ per monomer (out of 35 total Chls) and absorbs up to 750 nm ${ }^{[23,24]}$, while FRL-PSI binds 7-8 Chls $f$ per monomer (out of $90 \mathrm{Chls}$ ) and absorbs even up to $800 \mathrm{~nm}$. FaRLiP ${ }^{[22,23,25,26]}$ also involves the expression of new PBPs that are paralogues of the APC subunits expressed in WL ${ }^{[27]}$. These FRL-APC subunits, together with some of the WL-APC subunits, organize into bicylindrical cores (FRL-BCs) ${ }^{[27,28]}$. While the lowest energy 
pigments in the core of the WL-PBS emit at $680 \mathrm{~nm}$, the FRL-BCs contain more red-shifted phycobilins: their absorption peak is located around $710 \mathrm{~nm}$ and their terminal emitters at about $730 \mathrm{~nm}^{[24,27,28]}$. The organization of PBPs in FaRLiP organisms is species-dependent. Some strains possess only FRL-BCs in FRL ${ }^{[28]}$, while others can assemble the FRL-BCs to peripheral rods of PC and PE ${ }^{[29]}$. Finally, besides producing FRL-photosystems and FRL$B C s$, some strains were shown to maintain variable amounts of WL-photosystems and/or WL-PBS after weeks of acclimation to FRL $[23,24,27]$.

The use of red-shifted pigments represents a promising strategy for extending the photosynthetic active spectrum of other organisms, such as plants and algae. In order to achieve high biomass yields, however, the newly engineered photosynthetic units need to preserve high photochemical yields. In this respect, we have recently shown that the insertion of $\mathrm{Chl} f$ slows down charge separation in both photosystems and significantly decreases the efficiency of FRL-PSII in comparison to WL-PSII ${ }^{[23]}$. These reduced performances, especially in the case of FRL-PSII, can be ascribed to (i) a slower charge separation by the RCs due to a red-shifted primary electron donor and (ii) a less effective connectivity between the few Chls $f / d$ surrounded by a majority of Chls $a$. Little is known, however, about how efficiently the RCs process the photons harvested by the FRL-BCs, especially at physiological temperatures. This knowledge is particularly relevant because FRL-BCs absorb a substantial amount of FRL in the cells, implying that the fate of their excitations has a considerable impact on the overall performances. To shed more light on these aspects, we measured time-resolved fluorescence (TRF) of intact cells of two FaRLiP organisms acclimated to either WL of FRL. We performed experiments at two different excitation wavelengths to selectively excite the Chls or the bilins, thereby investigating the fate of the energy absorbed by either the photosystems or the PBPs. We use these data to quantify the efficiency of light conversion after exciting different units and, finally, we propose a structural model for the connectivity between the FRL-BCs and FRL-PSII that qualitatively accounts for the experimental findings. In the manuscript, we will only show the results from one of the two strains, Chroococcidiopsis thermalis (CT). The data of Chlorogloeopsis fritschii (CF), which are presented in the supplementary information and are fundamentally consistent with those from $C T$, will only be mentioned in the manuscript to highlight some relevant differences in the chromatic responses of the two strains.

\section{Materials and Methods}

\section{Cell cultures}

The strains Chlorogloepsis fritschi PCC 6912 (CF) and Chroococcidiopsis thermalis PCC 7203

(CT) were obtained from the Pasteur Culture Collection (Institut Pasteur, Paris, France) and grown at $30^{\circ} \mathrm{C}$ in $\mathrm{BG} 11^{[30]}$ medium with addition of $20 \mathrm{mM}$ HEPES-NaOH $(\mathrm{pH}=8.0)$. Both strains were grown under white light (WL) of $30 \mu \mathrm{mol}$ photons $\mathrm{m}^{-2} \mathrm{~s}^{-1}$ starting from $\mathrm{OD}_{750}$ 
$=0.1$ and collecting cells for experiments at $\mathrm{OD}_{750}=0.8-1.0$. The strains were also grown under far-red light (FRL, $738 \mathrm{~nm}$; Jazz) of $45 \mu \mathrm{mol}$ photons $\mathrm{m}^{-2} \mathrm{~s}^{-1}$ for 2.5 to 3 weeks prior to experiments, starting from $\mathrm{OD}_{750}=0.3-0.4$, with the medium being refreshed every week keeping an $\mathrm{OD}_{750}=1.0-1.5$. The cultures were grown in Erlenmeyer flasks shaking at $100 \mathrm{rpm}$.

\section{Steady-State Spectroscopy}

Room-temperature (RT) absorption spectra on cells were acquired on a Varian Cary 4000 UV-Vis-spectrophotometer (Agilent technologies) equipped with an integrating diffuse reflectance sphere (DRA-CA-50, Labsphere) to correct for light scattering. RT fluorescence spectra were acquired at an OD $<0.05 \mathrm{~cm}^{-1}$ at Qy maximum on a HORIBA Jobin-Yvon FluoroLog-3 spectrofluorometer. Absorption and fluorescence spectra were measured on at least three biological replicates for both FRL- and WL- acclimated cells, yielding similar results.

\section{Time-resolved fluorescence}

Time-resolved fluorescence (TRF) measurements with 400-nm excitation were performed as in Mascoli et al. ${ }^{[23]}$. TRF measurements with 577-nm excitation were recorded with a synchro scan streak camera setup ${ }^{[31,32]}$. In brief, the laser repetition rate was $3.8 \mathrm{MHz}$ and the time window $2.0 \mathrm{~ns}$. Measurements with nearly all PSII RCs open were performed with an excitation power of $0.2 \mu \mathrm{W}$ (with a sample volume of $2 \mathrm{~mL}$ ), while those with closed PSII $\mathrm{RCs}$ were performed at $2 \mu \mathrm{W}$, after addition of 3-(3,4-dichlorophenyl)-1,1-dimethylurea (DCMU) and pre-illumination with white light for one minute (with a sample volume of 1 $\mathrm{mL}$ ). The experiments were performed at RT in a magnetically stirred $1 \mathrm{~cm} \times 1 \mathrm{~cm}$ cuvette with a sample OD of about $0.5 \mathrm{~cm}^{-1}$ (excitation and detection from the sample at the edge of the cuvette, thereby avoiding self absorption) and a measuring time between 15 minutes and 1 hour of CCD exposure (no sample degradation was observed during the measuring time). The averaged images were corrected for background and shading, and then sliced into traces of $~ 1.5-\mathrm{nm}$ width prior to analysis. 400-nm excitation experiments consisted of three (two) biological replicas for FRL- (WL-) adapted cells, while 577-nm experiments consisted of two (one) biological replicas for FRL- (WL-) adapted cells. The biological replicas yielded very similar results, the only relevant difference between them being the amount of unconnected PBS, especially for cells of CF.

\section{Data analysis}

Fluorescence time traces were globally analyzed with Glotaran and the TIMP package for $\mathrm{R}^{[40]}$ using a number of parallel kinetic components. The total dataset can be described by the fitting function $f(t, \lambda)$ :

$$
f(t, \lambda)=\sum_{k=1}^{n} D A S_{k}(\lambda) \cdot \exp \left(-\frac{t}{\tau_{k}}\right) \otimes \operatorname{IRF}(t, \lambda)
$$


where each decay-associated spectrum $\left(D A S_{k}\right)$ is the amplitude factor associated with a decay component $k$ having a decay lifetime $\boldsymbol{\tau}_{k}$. The instrument response function $\operatorname{IRF}(t, \lambda)$ was estimated from the fitting (FWHM $\sim 20 \mathrm{ps}$ ) using a Gaussian profile. The fitting also accounts for time-zero dispersion. In some cases, a double Gaussian was required, consisting of a $\sim 20$ ps FWHM (90\% of IRF area) on top of a Gaussian of $\sim 100$ ps FWHM ( $10 \%$ of IRF area). For each experiment, the time-zero spectrum was obtained by summing all DAS. The steady-state fluorescence spectra were reconstructed by integrating the TRF data as:

$$
F(\lambda)=\sum_{k=1}^{n} D A S_{k}(\lambda) \cdot \tau_{k}
$$

In order to compare results from different measurements, the DAS and reconstructed steady-state fluorescence spectra were normalized to the area of the time-zero spectrum (corresponding to the same amount of initial excitation) in each dataset. In the following, we will mostly show the DAS resulting from the global analysis of TRF data. Overlays of the raw and fitted kinetic traces can be found in Figures S8-11.

\section{Results}

\section{Steady-state spectroscopy}

In comparison with cells acclimated to WL (WL-cells), CT cells acclimated to FRL (FRLcells) display an additional absorption band above $700 \mathrm{~nm}$ (Figure 1A). This is caused by the integration of $\mathrm{Chl} f$ in the FRL-photosystems and by the synthesis of red-shifted allophycocyanin (APC) forming bi-cylindrical cores (FRL-BCs) ${ }^{[17]}$. The extra absorption in the blue-green region $(\lambda<530 \mathrm{~nm}$ ) has been observed in other FaRLiP strain acclimated to $F R L$ and ascribed to an increased carotenoid content ${ }^{[1,25,33]}$. Furthermore, the relative decrease in the $560-630 \mathrm{~nm}$ region upon acclimation to FRL reflects a partial loss of the blue-most PBPs.
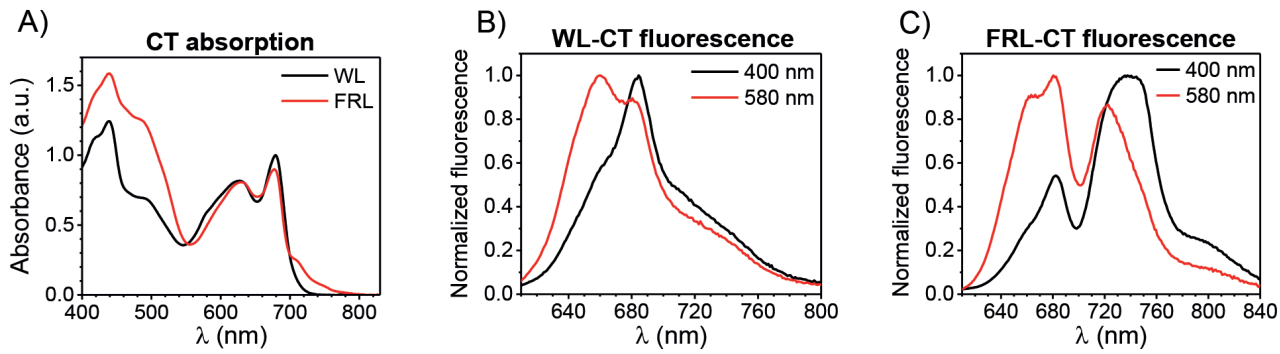

Figure 1. Steady-state spectroscopy of intact cells of CT acclimated to WL or FRL. A) Absorption spectra of WLand FRL-cells (normalized to the area in the region above $580 \mathrm{~nm}$ ). B) Fluorescence spectra of WL-cells excited at 400 and $580 \mathrm{~nm}$. C) Fluorescence spectra of FRL-cells excited at 400 and $580 \mathrm{~nm}$. 
Steady-state fluorescence of CT WL-cells excited at $400 \mathrm{~nm}$ (where Chls are prevalently excited) is dominated by WL-PSII emission peaking at $\sim 680 \mathrm{~nm}$ (Figure 1B, black line). Two shoulders are also observed, one at longer wavelengths due to WL-PSI and one at shorter wavelengths due to PBPs, which are excited to a minor extent at $400 \mathrm{~nm}$. Upon preferential PBS excitation (580 $\mathrm{nm}$, red line), the emission spectrum has two partially overlapping bands peaking at $\sim 660 \mathrm{~nm}$ (due to APC) and $\sim 680 \mathrm{~nm}$ (due to WL-PSII), whereas the relative contribution of WL-PSI at longer wavelengths decreases as compared to $400 \mathrm{~nm}$ excitation.

CT FRL-cells excited at $400 \mathrm{~nm}$ show two distinct fluorescence bands (Figure $1 \mathrm{C}$, black line). The less intense band peaking at $680 \mathrm{~nm}$ can be ascribed to WL-PSII maintained in $\mathrm{FRL}$, while the dominant band has a broad maximum near $740 \mathrm{~nm}$ and originates from the Chls $f$ in the FRL-photosystems. The short-wavelength side of this band, which is sensitized by $580 \mathrm{~nm}$ excitation, is mainly contributed to by the FRL-BCs ${ }^{[23]}$. The shoulder extending up to $800 \mathrm{~nm}$ stems from the extremely red-shifted Chls $f$ typical for FRL-PSI ${ }^{[22,23,26]}$. The $580 \mathrm{~nm}$ excitation is selective for the PBPs with a preference for PC, although APC is also excited. Two distinct fluorescence bands are observed also in this case (Figure $1 C$, red line). The short-wavelength band peaks at $680 \mathrm{~nm}$ (WL-PSII) with a shoulder at $660 \mathrm{~nm}$ due to APC and is reminiscent of the one observed in WL-cells upon $580 \mathrm{~nm}$ excitation (though the relative amplitudes at 660 and $680 \mathrm{~nm}$ are different). This means that a substantial amount of WL-PBS is still present in the FRL-cells as observed before in another FaRLiP strain ${ }^{\text {[24]. }}$ On the other hand, FRL-BCs harvest light for the FRL-photosystems, giving rise to the farred fluorescence band. The latter, however, is blue-shifted and sharper than upon $400 \mathrm{~nm}$ excitation due to a higher contribution from FRL-APC upon $580 \mathrm{~nm}$ excitation. The strong dependency of far-red fluorescence on the excitation wavelength demonstrates that FRL$\mathrm{BCs}$ and FRL-photosystems do not fully equilibrate before their excitations decay. The 800$\mathrm{nm}$ fluorescence is also substantially reduced when exciting at $580 \mathrm{~nm}$, suggesting that the excited PBPs are less well connected to FRL-PSI than to other photosynthetic units. Interestingly, the relative intensities of the visible and far-red emission bands upon 580 $\mathrm{nm}$ excitation are inverted in comparison to the $400 \mathrm{~nm}$ excitation case. Evidently, 580 $\mathrm{nm}$ excitation is more selective for the WL-PBSs that are preferentially connected to the WL-photosystems, while only a smaller fraction of the initial excitations is emitted by FRLBCs and FRL-photosystems. This is consistent with the absorption by the PC contained in WL-PBS being markedly higher than the absorption by FRL-BCs at $580 \mathrm{~nm}{ }^{[27,28]}$.

\section{Time-resolved fluorescence experiments}

Absorption and fluorescence measurements show that CT FRL-cells have a very heterogeneous photosynthetic apparatus. This includes both WL- and FRL-photosystems, together with WL-PBSs and FRL-BCs, which can give rise to a variety of associations. In order to investigate the connectivity between photosynthetic units and its effect on the 
photosynthetic performances, the excited-state dynamics of intact cells were probed via TRF (Figures 2-3, and S1-2). Cells were excited both at low power, when most PSII RCs are open, and at high power in the presence of DCMU, when PSII RCs are closed, to disentangle the spectroscopic signatures of PSII from those of PSI and estimate its photochemical yield. Experiments were performed at two different excitation wavelengths, $400 \mathrm{~nm}$ (more selective for the photosystems) and $577 \mathrm{~nm}$ (more selective for the PBPs). In this work we focus on the results from the 577-nm excitation experiments, while those from the 400 $\mathrm{nm}$ excitation experiments, which are the subject of our previous work ${ }^{[23]}$, are only used here to make relevant comparisons. A more detailed analysis of these data can be found in the supplementary information (Figures S1 and S2).

A)

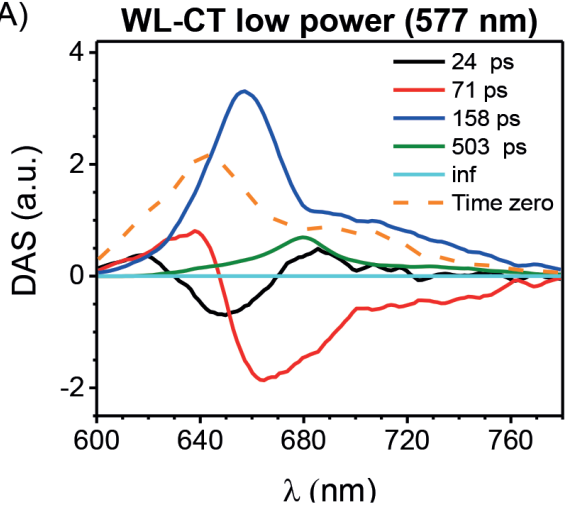

B)

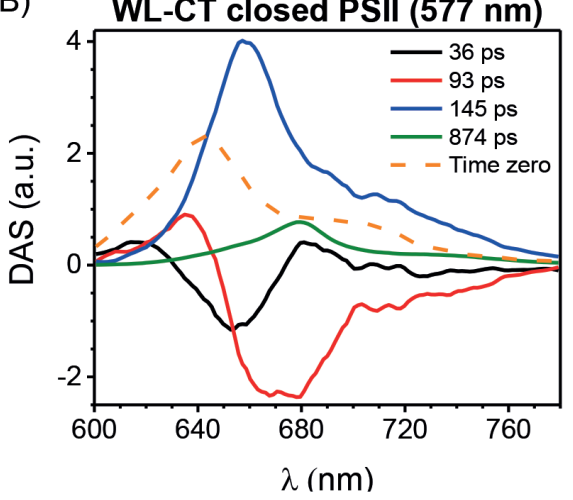

Figure 2. TRF measurements of WL-acclimated cells of CT. DAS from TRF measurements on WL-cells excited at $577 \mathrm{~nm}$ with (nearly) open (A) and closed (B) PSII RCs. The small long-lived (inf) component shown in (A) reflects some background signal. The time-zero spectrum (orange dashed lines) is calculated as the sum of all other DAS. The DAS are normalized to the area of the time-zero spectrum for better comparison.

\section{WL-Cells}

TRF data of WL-cells excited at $577 \mathrm{~nm}$ were fitted with four kinetic components (excluding the background signal, see Figure 2). The first three DAS (black, red and blue lines), whose lifetime and shape are very similar in open and closed state, stem mostly from PBPs. The first two DAS describe the funneling of excitations from the peripheral rods to the inner core of WL-PBS, occurring in less than $100 \mathrm{ps}$. The second DAS, with a negative amplitude above $680 \mathrm{~nm}$, might also include energy transfer from the PBS core to the Chls in WLPSII and WL-PSI. The third DAS (blue lines), with a lifetime of about 150 ps, is entirely positive and represents, therefore, an excited state decay. It is also very similar to the 150 ps DAS observed upon $400 \mathrm{~nm}$ excitation (Figure S1), suggesting a common origin for these components. These DAS can be largely ascribed to WL-PBS decay, although contributions from the two photosystems are probably present above $680 \mathrm{~nm}$. This decay is substantially shorter than in isolated PBS (typically ns) due to the connectivity of the PBS to PSI and PSII (photochemical quenching) ${ }^{[32,34]}$ in vivo. The lifetime of $\sim 150$ ps, which is 


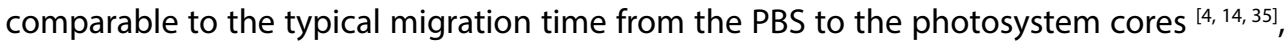
and the prevalent PBS contribution to these DAS, support the hypothesis that trapping of the PBS excitations by the RCs is to a large extent migration limited. This is particularly true for the WL-PBS connected to WL-PSI, as the latter can perform charge separation on a faster timescale than that of energy migration ( $30 \mathrm{ps}$, see Figure $\mathrm{S} 1)$. Furthermore, the weak dependency on the state of PSII RCs implies that the $\sim 150$ ps DAS largely stems from WL-PBSs that transfer excitations to WL-PSI. At the same time, some contribution from WL-PBS quenched by WL-PSII RCs might also be present, since the fluorescence decay of PSII in cyanobacteria is inherently multi-exponential and displays relatively short-lived components also in the closed state [36]. The slowest DAS (Figure 2, green lines), whose lifetime increases when PSII RCs close, can be attributed to WL-PSIl connected to WL-PBS. The lifetime of this DAS in open state ( $500 \mathrm{ps})$ is about $100 \mathrm{ps}$ longer than the lifetime of open WL-PSII measured after $400 \mathrm{~nm}$ excitation (below 400 ps, see Figure S1). This confirms that the diffusion of excitations from the periphery of the PBS to PSIl delays the trapping at the PSII RCs by 100 ps or more. On the other hand, the lifetime and spectra of the long-lived DAS for the closed state for both excitation wavelengths (Figure 4A) are very similar, indicating energy equilibration between WL-PBS and WL-PSII before excited state decay (i.e. within $1 \mathrm{~ns}$ ). The results presented so far for CT WL-cells (and those for CF WL-cells in Figures S4 and S5) are also consistent with those obtained previously for Synechocystis sp. PCC6803 ${ }^{[37,38]}$ and Synechococcus elongatus ${ }^{[32]}$.
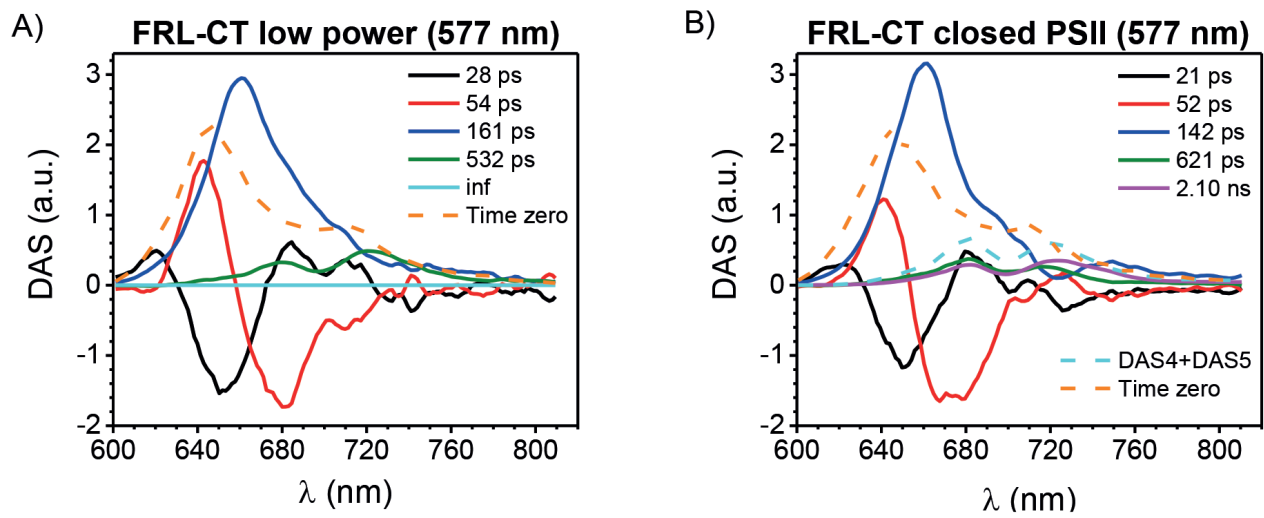

Figure 3. TRF measurements of FRL-acclimated cells of CT. DAS from TRF measurements on FRL-cells excited at $577 \mathrm{~nm}$ with (nearly) open (A) and closed (B) PSII RCs. The small long-lived (inf) component shown in (A) reflects some background signal. The time-zero spectrum (orange dashed lines) is calculated as the sum of all other DAS. The DAS are normalized to the area of the time-zero spectrum for better comparison.

\section{FRL-Cells}

According to the steady-state fluorescence spectra, FRL-cells contain WL-PBSs and FRL$\mathrm{BCs}$, which are both excited at $577 \mathrm{~nm}$ (with a preference for PC and, therefore, for the WLPBSs). Note that it is nearly impossible to excite FRL-BCs with a higher selectivity, as their 
absorption maxima (around $650 \mathrm{~nm}$ and $710 \mathrm{~nm}$ ) ${ }^{[21,28]}$ overlap with the absorption bands of the Chls (and with the detection window) to a much larger extent. After $577 \mathrm{~nm}$ excitation, four components were needed to globally fit the TRF data of FRL-cells with nearly all PSII RCs open (excluding the background signal, see Figure 3A), and five for the measurements on cells in closed state (Figure 3B). Similar to what was observed for WL-cells (Figure 2), the first two DAS (black and red lines) are dominated by downhill energy transfer in the WL-PBS and from them, possibly, to WL-PSII. Some energy transfer to far-red emitting units (FRL-BCs and FRL-photosystems) might also contribute to these components. The third DAS (blue lines), with a lifetime of $\sim 150 \mathrm{ps}$, is weakly sensitive to the state of the PSII RCs and reminiscent of the $\sim 150$ ps DAS observed in WL-cells (Figure 2, blue lines). This component largely represents photochemical quenching of WL-PBS excitations by the RCs of WL-photosystems (trapping by FRL-photosystems can be excluded, as it is substantially slower) ${ }^{[23]}$. Its amplitude relative to the time-zero spectrum, however, is reduced in FRL-cells (as the 577-nm laser also excites FRL-BCs, which decay more slowly and contribute to the longer-lived DAS, see below). The $\sim 150$ ps DAS in FRL-cells also have a lower relative amplitude above $700 \mathrm{~nm}$ and show a dip at about $725 \mathrm{~nm}$ (more evident in closed state, Figure 3B), indicating that some energy transfer to the FRL-units also takes place on a similar time scale. Some contribution from WL-PSII is probably present at $680 \mathrm{~nm}$, as the DAS amplitude at this wavelength decreases in favor of the longer-lived components when PSII RCs close (cfr blue and green/magenta lines in Figures $3 \mathrm{~A}$ and $\mathrm{BB}$ ). Both in open and closed state, the long-lived components are mostly associated with fluorescence from WL-PSII (peaking at $680 \mathrm{~nm}$ ) connected to WL-PBS and, at longer wavelengths, FRL-BCs and FRL-PSII. In open state, fluorescence from WL-PSII and FRL-BCs/FRL-PSII decays in about 500 ps (Figure 3A, green line), similar to what observed for WL-PSII in WL-cells. This lifetime is substantially shorter than the $\sim 900$ ps lifetime exhibited by FRL-PSII upon preferential Chl excitation ( $400 \mathrm{~nm}$, Figure S2A). Furthermore, the $\sim 500 \mathrm{ps}$ DAS obtained after $577 \mathrm{~nm}$ excitation peaks at $720 \mathrm{~nm}$ and is blue-shifted by $18 \mathrm{~nm}$ with respect to the $\sim 900$ ps DAS excited at $400 \mathrm{~nm}$ (Figure 4B) and by more than $25 \mathrm{~nm}$ with respect to the spectrum of isolated FRL-PSII of $C T{ }^{[23]}$. This indicates that FRL-BCs are the main contributors to the $\sim 500$ ps DAS excited at $577 \mathrm{~nm}$, while $\mathrm{Chl} f$ fluorescence dominates the $\sim 900$ ps DAS excited at $400 \mathrm{~nm}$. It is also evident that a large amount of FRL-BCs is functionally connected to FRL-PSII, since the average fluorescence lifetime at $720 \mathrm{~nm}$ upon 577-nm excitation clearly increases when PSII RCs close (Figure 4C). All these observations imply that trapping of FRL-APC excitations by FRL-PSII RCs is, on average, faster than after Chl excitation. In closed state, two long-lived DAS can be resolved after 577-nm excitation: one has a lifetime of about 600 ps (Figure 3B, green) and is enriched in WL-PSII and FRL-BCs, the other (magenta), with a lifetime above $2 \mathrm{~ns}$, contains contributions from WL-PSII, FRL-BCs and FRL-PSII. The amount of PBPs connected to FRL-PSI is likely to be small since all 577-nm excited DAS have a very low amplitude above $750 \mathrm{~nm}$. Overall, the observation that WL-PBSs remain preferentially connected to WL-photosystems, whereas FRL-BCs are coupled to FRLphotosystems (prevalently FRL-PSII) is in agreement with the spatial segregation between 
FRL- and WL-units observed in another FaRLiP strain after acclimation to FRL ${ }^{[39]}$. Notably, both long-lived DAS for the closed state (577-nm excitation) are significantly blue-shifted with respect to the long-lived DAS observed after 400-nm excitation (also in closed state, see Figure 4D). The same effect is observed for the open state (Figure 4B). This confirms that, even if FRL-BCs are functionally connected to FRL-PSII (as they display variable fluorescence), the two units do not fully equilibrate even when the PSII RCs are closed and the excitedstate lifetime is much longer (up to 2 ns).
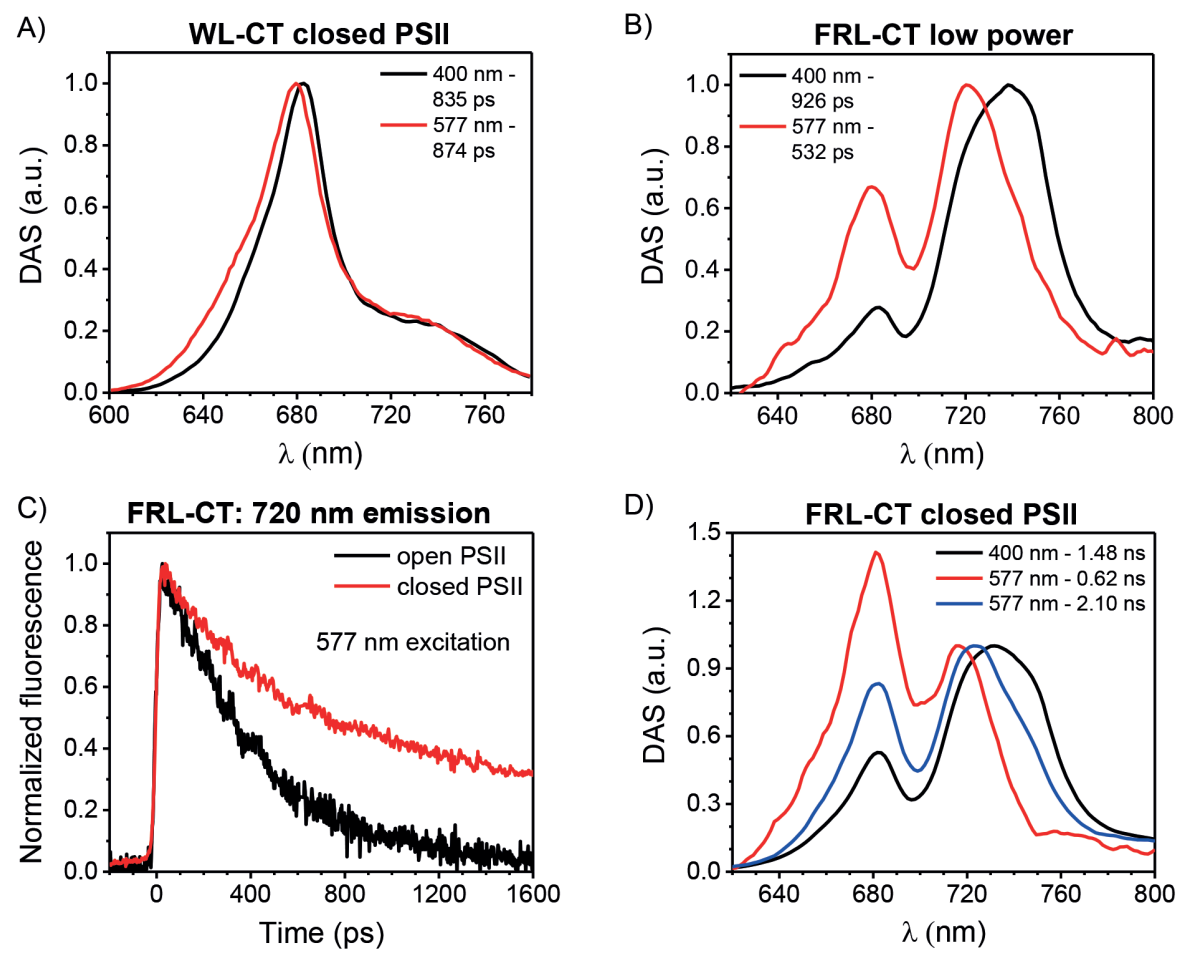

Figure 4. TRF measurements of WL- and FRL-acclimated cells of CT. A) Normalized long-lived DAS from TRF measurements of WL-cells with closed PSII RCs after 400 and $577 \mathrm{~nm}$ excitation (corresponding to the green DAS in Figures S1B and 2B). B) Normalized long-lived DAS from TRF measurements of FRL-cells with nearly all PSII RCs open after 400 and $577 \mathrm{~nm}$ excitation (corresponding to the green DAS in Figures S2A and 3A). C) Normalized fluorescence traces detected at $720 \mathrm{~nm}$ after $577 \mathrm{~nm}$ excitation of FRL-cells with open and closed PSII RCs. D) Long-lived DAS (normalized to the peak in the far-red region) from TRF measurements of FRL-cells with closed PSII RCs after 400 and $577 \mathrm{~nm}$ excitation (corresponding to the green DAS in Figure S2B and to the green and magenta DAS in Figure 3B).

Efficiency of PSII photochemistry under different conditions. The TRF data measured under different PSII RC conditions can be integrated to obtain steady-state fluorescence spectra in open $\left(F_{o}\right)$ and closed $\left(F_{m}\right)$ state and calculate the variable fluorescence $\left(F_{v}=\right.$ $F_{m}-F_{o}$ ) at various emission wavelengths (Figure 5). The variable fluorescence spectrum is highly informative of the connectivity between photosynthetic units because it only stems from the pigments that transfer energy to the PSII RCs. Furthermore, assuming that 
the contribution of PSI fluorescence can be neglected in the spectral regions where PSII emission is maximal, the ratio $F_{v} / F_{m}$ in these regions can be used to estimate the efficiency of PSII photochemistry, $\Phi_{\text {PSII }}$

When PSII RCs close, WL-cells show a relative increase in the emission at $680 \mathrm{~nm}$, where the contribution of WL-PSII is maximal. This effect is observed at both excitation wavelengths (Figures 5A-B). The variable fluorescence spectrum of WL-cells essentially reproduces that of WL-PSIl but is enriched at shorter wavelengths due to the WL-PBS. Consequently, $F_{v} / F_{m}$ has a maximum of about 0.6 around $680 \mathrm{~nm}$ and decreases at longer wavelengths, where WL-PSI emission is stronger, and at shorter wavelengths, due to the to the slower trapping of the excitation formed in the WL-PBSs by the PSII RCs and, possibly, some WL-PBSs that are not connected to WL-PSII (Figure 5C). Similar $F_{v}$ spectra are obtained upon $400 \mathrm{~nm}$ and $577 \mathrm{~nm}$ excitation, but the extent of variable fluorescence is clearly lower after PBP excitation. As a result, the maximal $\mathrm{F}_{\mathrm{v}} / \mathrm{F}_{\mathrm{m}}$ drops from 0.58 upon preferential $\mathrm{Chl}$ excitation to 0.41 upon PBP excitation (Figure 5C). This difference is the result of two simultaneous effects: (i) only a fraction of WL-PBS transfer energy to WL-PSII and (ii) trapping by WL-PSII after PBS excitation is slower than trapping after direct WL-PSII excitation due to the finite time required for energy migration from the WL-PBS to WL-PSII. This means that, in WLcells, WL-PBSs increase the absorption cross-section of WL-PSIl at the expense of some photochemical efficiency.
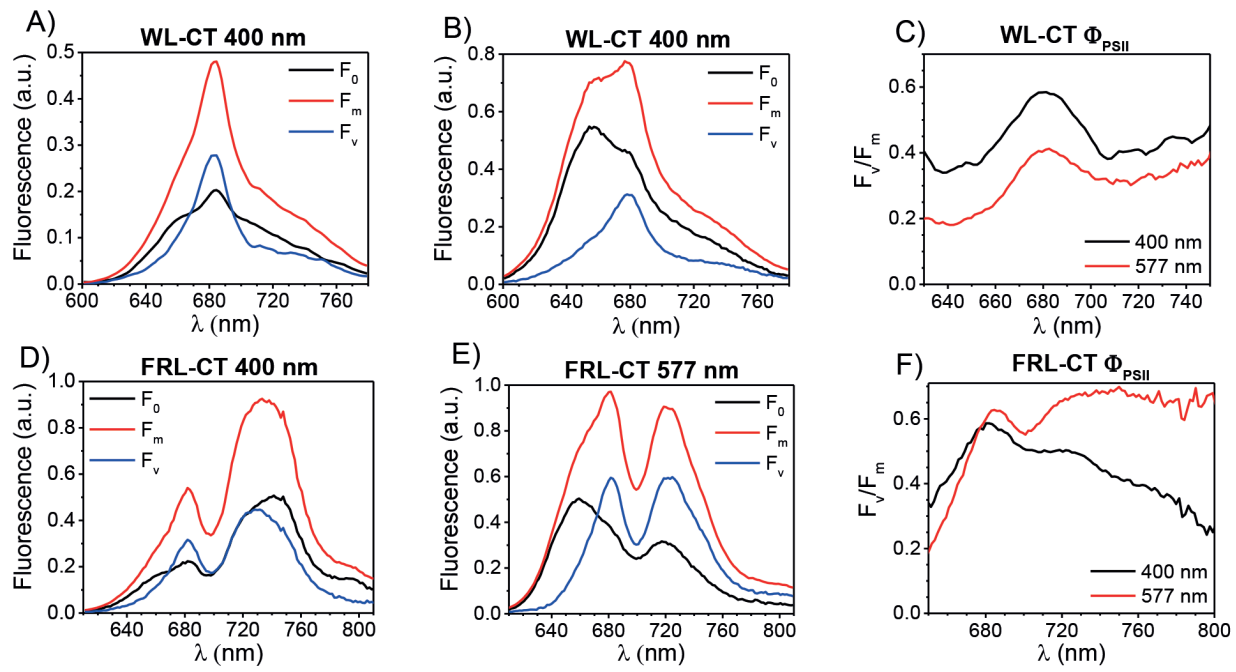

Figure 5. Variable fluorescence and PSII photochemical efficiency of WL-and FRL-cells of CT excited at different wavelengths. A,B) Steady-state fluorescence spectra of WL-cells in open $\left(F_{o}\right)$ and closed $\left(F_{m}\right)$ state excited at 400 $\mathrm{nm}(\mathrm{A})$ and $577 \mathrm{~nm}(\mathrm{~B})$ obtained from the DAS in Figures S1 and 2, respectively. Variable fluorescence spectra $\left(\mathrm{F}_{\mathrm{v}}\right)$ are calculated as the difference between $F_{m}$ and $\left.F_{0} . C\right) F_{v} / F_{m}$ of WL-cells at different wavelengths calculated from the spectra in $(A)$ and $(B)$. $D, E)$ Steady-state fluorescence spectra of FRL-cells in open $\left(F_{o}\right)$ and closed $\left(F_{m}\right)$ state excited at $400 \mathrm{~nm}(D)$ and $577 \mathrm{~nm}$ (E) obtained from the DAS in Figures S2 and 3, respectively. F) $F_{v} / F_{m}$ of FRL-cells at different wavelengths calculated from the spectra in (D) and (E). 
The time-integrated spectra of FRL-cells are highly congested due to the heterogeneity of their photosynthetic apparatus, particularly in the far-red region, where the contributions of WL-PSI, FRL-BCs, FRL-PSII, and FRL-PSI overlap substantially. In the following analysis, we will neglect the contributions from both types of PSI and use the steady-state $F_{\circ}$ and $F_{m}$ spectra to estimate $\Phi_{\text {FRL-PSII }}$. The validity of this approximation is supported by the fact that very similar results are obtained when the short-lived components from the TRF data (where the contribution from PSI is dominant) are excluded from the calculation (Figure S3). The variable fluorescence of FRL-cells shows two distinct peaks (Figure 5DE): one around $680 \mathrm{~nm}$, which we ascribe to WL-PSII and its WL-PBS antenna, and one in the far-red due to FRL-PSII and its FRL-BC antenna. Upon $400 \mathrm{~nm}$ excitation (Figure 5D), the $F_{v}$ spectrum in the far-red region is clearly blue-shifted with respect to both $F_{o}$ and $\mathrm{F}_{\mathrm{m}}$ and also relative to the spectrum of isolated FRL-PSII ${ }^{[23]}$. This indicates that trapping by FRL-PSII is more efficient at shorter wavelengths, where the contribution of FRL-BCs is larger. Upon $400 \mathrm{~nm}$ excitation, $F_{v} / F_{m}$ is therefore 0.50 at $720 \mathrm{~nm}$ (Figure 5F), it drops to about 0.44 at $740-750 \mathrm{~nm}$, where fluorescence from the Chls $f$ of FRL-PSII is maximal, and decreases even more at longer wavelengths, where FRL-PSI fluorescence dominates. Upon $577 \mathrm{~nm}$ excitation, as a consequence of the larger contribution from FRL-BCs, $\mathrm{F}_{\mathrm{o}^{\prime}}$ $F_{m}$ and $F_{v}$ all peak around $720 \mathrm{~nm}$ in the far-red region (Figure $5 E$ ). In addition, $F_{v} / F_{m}$ at $\lambda$ $>700 \mathrm{~nm}$ is substantially higher than after $400 \mathrm{~nm}$ excitation, rising to 0.68 (Figure 5F). This implies that FRL-PSII photochemistry after FRL-BCs excitation is more efficient than after direct Chl excitation. The opposite is normally observed for WL-PSIl of cyanobacteria ${ }^{[40]}$. The main cause of this somehow counter-intuitive finding is that trapping by FRL-PSII after excitation of FRL-BCs (requiring about 500 ps) is remarkably faster than after Chl excitation (requiring about $900 \mathrm{ps}$ ).

The spectroscopic data from WL- and FRL-cells of CF are shown in Figures S4-8 and are consistent with those presented above for $C T$. The major difference between these two strains is that CF contains a higher amount of PBPs/PBSs that are not functionally connected to the photosystems. The reason for retaining a conspicuous amount of functionally unconnected PBS in this strain is not known yet, but they might constitute a nitrogen reserve. This feature is common to both WL- and FRL-acclimated cells of CF and is responsible for a reduced $\mathrm{F}_{\mathrm{v}} / \mathrm{F}_{\mathrm{m}}$ when the PBPs are selectively excited (in comparison to $C T$ ). As pointed out in our previous work, FRL-cells of CF also retain a substantially

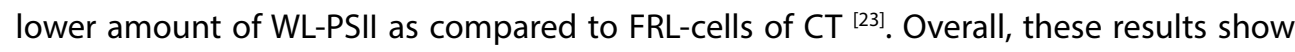
that, although the photosynthetic complexes of CF and CT display different degrees of heterogeneity upon FaRLiP, the association between FRL-BCs and FRL-PSIl gives rise to a similar energy landscape and excited-state kinetics. 


\section{Discussion}

\section{Excitations formed in FRL bicylindrical cores use a shortcut to reach the RCs of FRL-PSII}

Our experimental results can be summarized as follows:

- After several weeks of acclimation to $F R L$, the cells have a very heterogeneous photosynthetic apparatus comprising FRL- and WL-photosystems, FRL-BCs and WLPBSs.

- In WL-cells, the PSII RCs trap excitations formed in the adjacent PBSs more slowly (and, therefore, less efficiently) than the excitations formed directly on the Chls. Furthermore, excitations in the WL-PBSs and WL-PSII equilibrate to a large extent before decaying.

- Conversely, FRL-BCs can deliver excitations to the RC of FRL-PSII and power charge separation with a higher rate and efficiency than at least a fraction of the Chls $f$ found in the FRL-PSII antenna (CP43 and CP47 paralogs). Furthermore, the excitations formed in the FRL-BCs do not equilibrate entirely with the Chls $f$ of FRL-PSII, even after $2 \mathrm{~ns}$.

The lack of energy equilibration is usually ascribed to disrupted energetic connectivity between pigments. However, FRL-BCs display variable fluorescence $F_{v}$ and their excitations are trapped within 500 ps, implying that they are energetically well connected to the RCs of FRL-PSII. The only way to account for these seemingly contrasting results is to assume that, for structural reasons, the energy transfer route from FRL-BCs to the RC of FRL-PSII is somehow faster than that connecting some of the Chls $f$ in the antenna and the RC itself. This would also explain why excitations in the FRL-BCs can reach the RC but not all Chls $f$ in the FRL-PSIl antenna, resulting in the lack of energy equilibration between the two units. The shortcut connecting the FRL-BCs to the FRL-PSII RCs is likely to involve an intermediate Chl in the antenna (CP43/47 paralogs) acting as a bridge. Indeed, structural data on canonical PBSs have shown that the pigments in the APC core are too far from those of the PSII RC to allow direct energy transfer between them ${ }^{[7,9,41]}$. Since the trapping in the RCs of FRL-PSII after PBP excitation is faster (on average) than after Chl excitation, it is clear that the bridging $\mathrm{Chl}$ in the antenna must be better connected to the RC than some of the Chls $f$. In order to avoid energetic barriers, it is reasonable that this pivotal $\mathrm{Chl}$ accepting excitations from farred emitting APC is also red-shifted (i.e. it is either a $\mathrm{Chl} d$ or $f$ ). 


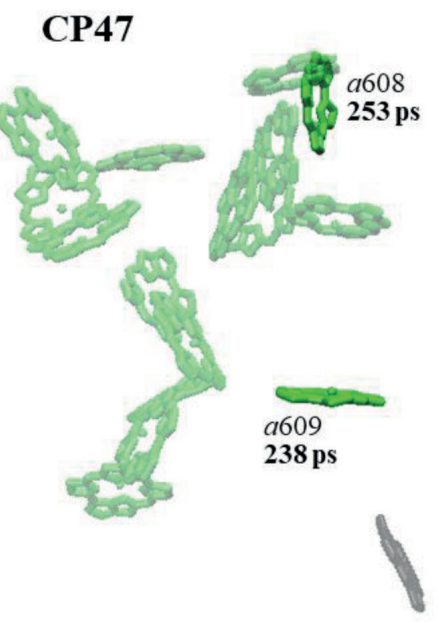

RC

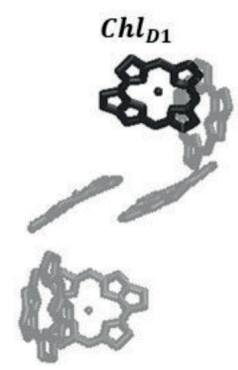

N

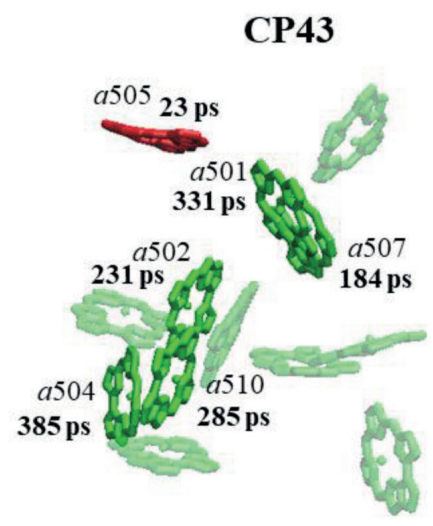

Figure 6. Hopping times between potential binding sites for red-shifted Chls in the antenna and the primary donor $\mathrm{Chl}_{\mathrm{D} 1}$. The $\mathrm{Chl}$ binding sites are taken from the PSIl structure of $T$. vulcanus by Umena et al. [42]. The hopping times (inverse of energy transfer rates) are taken from Mascoli et al. [23] and are calculated in the case where the energy donor in the antenna is a Chl f emitting at $740 \mathrm{~nm}$ (based on the average emission wavelength of FRLPSII) and the acceptor ( $\mathrm{Chl}_{\mathrm{D} 1}$ ) is a Chl f absorbing at $727 \mathrm{~nm}$ (based on Nurnberg et al.) [22]. The RC pigments are shown in transparent black, and those in the antenna (CP43/47) in transparent green. Chl is shown in solid black, while the antenna Chls with a hopping time below 500 ps are in solid green. Chl505, which exhibits the fastest energy transfer towards $\mathrm{ChI}_{\mathrm{D} 1^{\prime}}$, is colored in red.

All these findings further support our previous hypothesis ${ }^{[23]}$ that the Chls $f / d$ of FRL-PSII are generally not well connected to each other and to the RC. Indeed, trapping by FRL-PSII is drastically slower than in WL-PSII due to two concurring effects: (i) the slower charge separation by the FRL-PSII RCs and (ii) the slower energy migration towards the RCs caused by a less effective connectivity between pigments. The latter is mainly a consequence of the small number of red-shifted pigments surrounded by a majority of Chls $a$. In this view, the few Chls $f / d$ in the antenna can promptly trap the excitations from the surrounding Chls $a$ but, for charge separation to occur, they need to transfer them to the single redshifted pigment found in the RC (either a Chl $f$ or $d$ absorbing at $727 \mathrm{~nm}$ ), most likely at the position of the primary donor, $\mathrm{Chl}_{\mathrm{D} 1}{ }^{[22]}$. These conditions drastically reduce the connectivity between the antenna and the $\mathrm{RC}$, as most antenna Chls are only weakly coupled to $\mathrm{Chl}_{\mathrm{D} 1}$. In the above discussion, however, we inferred that at least one red-shifted $\mathrm{Chl}$ in the antenna, acting as an energy conduit between FRL-PSII and FRL-BCs, should be effectively connected to the RC. This is in agreement with our previous measurements on purified FRL-PSII, whose fluorescence decay in open state is multi-exponential: this can be interpreted in terms of the RCs trapping excitations from different red-shifted Chls in the antenna on different timescales, from 100-200 ps to about $1 \mathrm{~ns}$. $\mathrm{Chl}_{\mathrm{D} 1}$ is spatially closer to $\mathrm{CP} 43^{[42]}$ and should therefore trap excitations from its Chls $\mathrm{f} / \mathrm{d}$ more rapidly than from CP47 (if any). Based on the calculated couplings, only one binding site of CP43 (Chl505; 
nomenclature based on the structure with PDB code $3 W U 2^{[42]}$ ) should be able to transfer excitations to $\mathrm{Chl}_{\mathrm{D} 1}$ in less than $100 \mathrm{ps}$ (if occupied by a red-shifted $\mathrm{Chl}$ ), whereas five more Chls of CP43 and two of CP47 could transfer in 200-400 ps (if Chls f/d). By contrast, the migration time in canonical PSII is in the order of 50 ps or less ${ }^{[43]}$, with no large differences between $\mathrm{CP} 43$ and $\mathrm{CP} 47^{[44]}$. The total fluorescence lifetime is then obtained by summing the migration time and the trapping time, which is also significantly longer than in WL-PSII. In this view, the lifetime heterogeneity of FRL-PSII can be explained by a faster trapping of the excitations from the Chls $f$ of CP43 and a slower trapping from the Chls $f$ of CP47. Furthermore, Chl505 of CP43 is located at the stromal side of the complex, where the PBS basal core cylinder attaches to PSII. Being also the most strongly coupled pigment to $\mathrm{Chl}_{\mathrm{D} 1}$, it is therefore the ideal candidate for bridging the FRL-BCs to the RCs of FRL-PSII, resulting in the relatively fast trapping observed after PBP excitation. This hypothesis is also consistent with structural data/models of supercomplexes of PBS and (canonical) PSII, indicating that the terminal emitters of the PBS, located in the ApcE subunit, are spatially closer to the Chls of CP43 than those of CP47 ${ }^{[9,45]}$. Finally, the weak connectivity between the Chls of CP43 and those of CP47 might account for the lack of equilibration observed in the TRF measurements. The evidence that, after $577 \mathrm{~nm}$ excitation, the emission in open state is dominated by APC might result from a kinetics where the bridging red-shifted $\mathrm{Chl}$ receives excitations from $A P C$ only for a short transient before transferring them to the RC. When PSII RCs are closed, the excitations formed in the PBPs would probably remain localized on the FRL-BCs and CP43 (with a preference for APC due to the larger amount of pigments), without reaching the Chls $f$ of CP47.

\section{Conclusions}

The FaRLiP strains investigated in this work possess a highly heterogeneous photosynthetic machinery when acclimating to FRL. Together with FRL-photosystems, incorporating redshifted Chls, and FRL-BCs, containing red-shifted APC, they also maintain considerable amounts of WL-photosystems and WL-PBSs. TRF measurements demonstrate that WL-PBS remain to a large extent coupled to WL-photosystems, whereas FRL-BCs transfer energy to FRL-PSII mostly. In comparison to the association between WL-PBSs and WL-PSII, the one between FRL-BCs and FRL-PSII shows two unique spectroscopic features: first, trapping of the excitations formed in the FRL-BC antenna is, on average, faster than trapping of Chl excitations formed in the photosystem cores; second, the excitations on the phycobilins do not fully equilibrate with the Chls even after $2 \mathrm{~ns}$. These two remarkable results imply that the excitations formed in the FRL-BCs can use a shortcut to reach the RCs faster than from some of the red-shifted Chls in the FRL-PSII antenna. Furthermore, different pools of Chls $f$ coexist in FRL-PSII, which cannot transfer excitations between each other. An appealing hypothesis that rationalizes these findings and is supported by structural data 
is that FRL-BCs are energetically connected to the FRL-PSII RC via a specific red-shifted ChI of CP43 that shows the largest coupling to $\mathrm{Chl}_{\mathrm{D} 1}$.

The result of this peculiar energetics and pigment connectivity is that trapping by FRLPSII RCs has higher photochemical yields when exciting the PBPs than when exciting the Chls directly. Evidently, the positioning of the red-shifted pigments has been optimized for FRL-PSII to be more efficient when associated with FRL-BCs rather than being on its own. This would also be advantageous in terms of absorption cross-sections in FRL, as the FRL-BCs carry the largest number of far-red absorbing pigments. This observation might partially explain why in the absence of FRL-BCs, FRL-PSIl does not accumulate in FaRLiP organisms ${ }^{[46]}$. 


\section{Literature}

1. Glazer, A.N., Phycobilisome a macromolecular complex optimized for light energy transfer. Biochimica et Biophysica Acta (BBA)-Reviews on Bioenergetics, 1984. 768(1): p. 29-51.

2. Sidler, W.A., Phycobilisome and phycobiliprotein structures, in The molecular biology of cyanobacteria. 1994, Springer. p. 139-216.

3. Watanabe, M. and M. Ikeuchi, Phycobilisome: architecture of a light-harvesting supercomplex. Photosynthesis research, 2013. 116(2): p. 265-276.

4. Bryant, D.A. and D.P. Canniffe, How nature designs light-harvesting antenna systems: design principles and functional realization in chlorophototrophic prokaryotes. Journal of Physics B: Atomic, Molecular and Optical Physics, 2018. 51(3): p. 033001.

5. Glazer, A.N., Light guides: directional energy transfer in a photosynthetic antenna. Journal of Biological Chemistry, 1989. 264(1): p. 1-4.

6. Dong, C., et al., ApcD is necessary for efficient energy transfer from phycobilisomes to photosystem $I$ and helps to prevent photoinhibition in the cyanobacterium Synechococcus sp. PCC 7002. Biochimica et Biophysica Acta (BBA)-Bioenergetics, 2009. 1787(9): p. 1122-1128.

7. Liu, H., et al., Phycobilisomes supply excitations to both photosystems in a megacomplex in cyanobacteria. Science, 2013. 342(6162): p. 1104-1107.

8. Arteni, A.A., G. Ajlani, and E.J. Boekema, Structural organisation of phycobilisomes from Synechocystis sp. strain PCC6803 and their interaction with the membrane. Biochimica et Biophysica Acta (BBA)-Bioenergetics, 2009. 1787(4): p. 272-279.

9. Chang, L., et al., Structural organization of an intact phycobilisome and its association with photosystem II. Cell research, 2015. 25(6): p. 726-737.

10. Schirmer, T., W. Bode, and R. Huber, Refined three-dimensional structures of two cyanobacterial C-phycocyanins at 2.1 and $2.5 \AA$ resolution: a common principle of phycobilin-protein interaction. Journal of molecular biology, 1987. 196(3): p. 677-695.

11. Reuter, W., et al., Structural analysis at $2.2 \AA$ of orthorhombic crystals presents the asymmetry of the allophycocyanin-linker complex, APQ LC7. 8, from phycobilisomes of Mastigocladus laminosus. Proceedings of the National Academy of Sciences, 1999. 96(4): p. 1363-1368.

12. Gindt, Y.M., et al., Core mutations of Synechococcus sp. PCC 7002 phycobilisomes: a spectroscopic study. Journal of Photochemistry and Photobiology B: Biology, 1992. 15(1-2): p. 75-89.

13. Gindt, Y.M., et al., Spectroscopic studies of phycobilisome subcore preparations lacking key core chromophores: assignment of excited state energies to the $L \mathrm{Cm}, \beta 18$ and $A A P-B$ chromophores. Biochimica et Biophysica Acta (BBA)-Bioenergetics, 1994. 1186(3): p. 153-162.

14. van Grondelle, R., et al., Energy transfer and trapping in photosynthesis. Biochimica et Biophysica Acta (BBA)-Bioenergetics, 1994. 1187(1): p. 1-65.

15. Bjijrn, L., et al., viewpoint: Why chlorophyll a. Photosynth. Res, 2009. 99: p. 85-98.

16. Chen, M., et al., A cyanobacterium that contains chlorophyll $f$-a red-absorbing photopigment. FEBS letters, 2012. 586(19): p. 3249-3254. 
17. Gan, F., et al., Extensive remodeling of a cyanobacterial photosynthetic apparatus in far-red light. Science, 2014. 345(6202): p. 1312-1317.

18. Gan, F., G. Shen, and D.A. Bryant, Occurrence of far-red light photoacclimation (FaRLiP) in diverse cyanobacteria. Life, 2015. 5(1): p. 4-24.

19. Gan, F. and D.A. Bryant, Adaptive and acclimative responses of cyanobacteria to far-red light. Environmental microbiology, 2015. 17(10): p. 3450-3465.

20. Ho, M.-Y., et al., Light-dependent chlorophyll $f$ synthase is a highly divergent paralog of PsbA of photosystem II. Science, 2016. 353(6302).

21. Ho, M., et al., Light regulation of cyanobacterial pigment and photosystem biosynthesis. Curr. Opin. Plant Biol., 2017. 37: p. 24-33.

22. Nürnberg, D.J., et al., Photochemistry beyond the red limit in chlorophyll f-containing photosystems. Science, 2018. 360(6394): p. 1210-1213.

23. Mascoli, V., L. Bersanini, and R. Croce, Far-red absorption and light-use efficiency trade-offs in chlorophyll f photosynthesis. Nature Plants, 2020. 6(8): p. 1044-1053.

24. Ho, M.-Y., et al., Extensive remodeling of the photosynthetic apparatus alters energy transfer among photosynthetic complexes when cyanobacteria acclimate to far-red light. Biochimica et Biophysica Acta (BBA)-Bioenergetics, 2020. 1861(4): p. 148064.

25. Li, Y., N. Vella, and M. Chen, Characterization of isolated photosystem I from Halomicronema hongdechloris, a chlorophyll f-producing cyanobacterium. Photosynthetica, 2018. 56(1): p. 306315.

26. Kurashov, V., et al., Energy transfer from chlorophyll $f$ to the trapping center in naturally occurring and engineered Photosystem I complexes. Photosynthesis research, 2019. 141(2): p. 151-163.

27. Ho, M.-Y., et al., Far-red light photoacclimation (FaRLiP) in Synechococcus sp. PCC 7335. II. Characterization of phycobiliproteins produced during acclimation to far-red light. Photosynthesis research, 2017. 131(2): p. 187-202.

28. Li, Y., et al., Characterization of red-shifted phycobilisomes isolated from the chlorophyll f-containing cyanobacterium Halomicronema hongdechloris. Biochimica et Biophysica Acta (BBA)-Bioenergetics, 2016. 1857(1): p. 107-114.

29. Soulier, N., T.N. Laremore, and D.A. Bryant, Characterization of cyanobacterial allophycocyanins absorbing far-red light. Photosynthesis Research, 2020. 145(3): p. 189-207.

30. Rippka, R., et al., Generic assignments, strain histories and properties of pure cultures of cyanobacteria. Microbiology, 1979. 111 (1): p. 1-61.

31. Choubeh, R.R., et al., State transitions in the cyanobacterium Synechococcus elongatus 7942 involve reversible quenching of the photosystem II core. Biochimica et Biophysica Acta (BBA)Bioenergetics, 2018. 1859(10): p. 1059-1066.

32. Bhatti, A.F., et al., State transitions in cyanobacteria studied with picosecond fluorescence at room temperature. Biochimica et Biophysica Acta (BBA) - Bioenergetics, 2020. 1861(10): p. 148255.

33. Schmitt, F.-J., et al., Photosynthesis supported by a chlorophyll f-dependent, entropy-driven uphill energy transfer in Halomicronema hongdechloris cells adapted to far-red light. Photosynthesis research, 2019. 139(1): p. 185-201. 
34. Tian, L., et al., Site, Rate, and Mechanism of Photoprotective Quenching in Cyanobacteria. Journal of the American Chemical Society, 2011. 133(45): p. 18304-18311.

35. Akhtar, P., et al., Time-resolved fluorescence study of excitation energy transfer in the cyanobacterium Anabaena PCC 7120. Photosynthesis research, 2020. 144(2): p. 247.

36. Tian, L., S. Farooq, and H. van Amerongen, Probing the picosecond kinetics of the photosystem II core complex in vivo. Physical chemistry chemical physics, 2013. 15(9): p. 3146-3154.

37. Krumova, S., et al., Monitoring photosynthesis in individual cells of Synechocystis sp. PCC 6803 on a picosecond timescale. Biophysical journal, 2010. 99(6): p. 2006-2015.

38. Gottmann, P., et al., A computational biology approach of a genome-wide screen connected miRNAs to obesity and type 2 diabetes. Molecular metabolism, 2018. 11: p. 145-159.

39. Majumder, E.L.-W., et al., Subcellular pigment distribution is altered under far-red light acclimation in cyanobacteria that contain chlorophyll f. Photosynthesis research, 2017. 134(2): p. 183-192.

40. Remelli, W. and S. Santabarbara, Excitation and emission wavelength dependence of fluorescence spectra in whole cells of the cyanobacterium Synechocystis sp. PPC6803: Influence on the estimation of photosystem II maximal quantum efficiency. Biochimica et Biophysica Acta (BBA)Bioenergetics, 2018. 1859(11): p. 1207-1222.

41. Krasilnikov, P.M., D.V. Zlenko, and I.N. Stadnichuk, Rates and pathways of energy migration from the phycobilisome to the photosystem II and to the orange carotenoid protein in cyanobacteria. FEBS letters, 2020. 594(7): p. 1145-1154.

42. Umena, Y., et al., Crystal structure of oxygen-evolving photosystem II at a resolution of $1.9 \AA$. Nature, 2011. 473(7345): p. 55-60.

43. van der Weij- de Wit, C., et al., Charge separation is virtually irreversible in photosystem II core complexes with oxidized primary quinone acceptor. The Journal of Physical Chemistry A, 2011. 115(16): p. 3947-3956.

44. Raszewski, G. and T. Renger, Light harvesting in photosystem II core complexes is limited by the transfer to the trap: can the core complex turn into a photoprotective mode? Journal of the American Chemical Society, 2008. 130(13): p. 4431-4446.

45. Zlenko, D., P.M. Krasilnikov, and I.N. Stadnichuk, Structural modeling of the phycobilisome core and its association with the photosystems. Photosynthesis research, 2016. 130(1): p. 347-356.

46. Bryant, D.A., et al., Far-red light allophycocyanin subunits play a role in chlorophyll d accumulation in far-red light. Photosynthesis research, 2020. 143(1): p. 81-95. 


\section{Supplementary Information}

A)

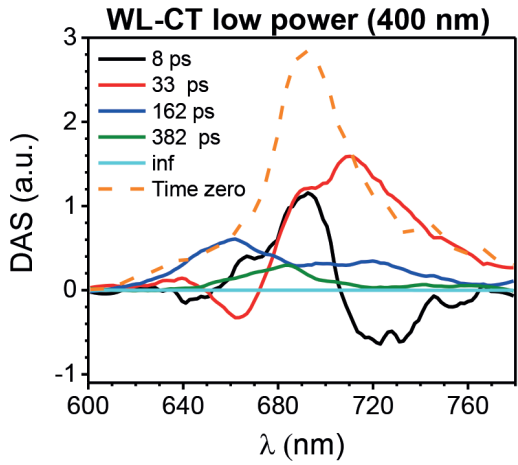

B)

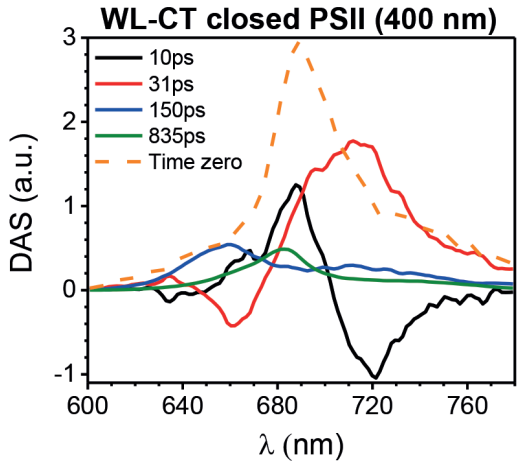

Supplementary Figure 1. TRF measurements of WL-acclimated cells of CT excited at $400 \mathrm{~nm}$. DAS from TRF measurements on WL-cells excited at $400 \mathrm{~nm}$ with (nearly) open (A) and closed (B) PSII RCs (taken from Mascoli et al) [1]. The small long-lived (inf) component shown in (A) reflects some background signal. The time-zero spectrum (orange dashed lines) is calculated as the sum of all other DAS. The DAS are normalized to the area of the time-zero spectrum for better comparison.

The two fastest DAS (black and red lines), with a lifetime of about 10 and $30 \mathrm{ps,} \mathrm{respectively,}$ are nearly independent of the state of the PSII RCs, and can be largely ascribed to downhill energy transfer and trapping by WL-PSI. At shorter wavelengths, smaller band shift features due to downhill energy transfer in the WL-PBS are also observed (as PBPs are excited to a minor extent at this wavelength). The $\sim 150$ ps DAS (blue lines) is entirely positive and stems prevalently from the decay of the excitations in the WL-PBS, though its amplitude at $680 \mathrm{~nm}$ slightly decreases in favor of the longer-lived component when PSII RCs close, indicating some contribution from WL-PSII. The long-lived component (green lines), whose lifetime and amplitude increase in closed state, can be attributed to WLPSII, with some WL-PBS contribution at shorter wavelengths. The ratio between the area of the long-lived PSII DAS and that of the time-zero spectrum in closed state represents a low estimate for the amount of initial excitations that are emitted by WL-PSII (as the shorter-lived DASs also contain minor contributions from WL-PSII). The amplitude of this DAS is much smaller than that of the 30 ps DAS (representing WL-PSI decay) and accounts for only $15 \%$ of the total initial excitations (based on the area of the time-zero spectrum, orange dashed lines). This is consistent with the evidence that PSI commonly binds the majority of total Chls in cyanobacterial cells ${ }^{[2,3]}$. Conversely, after 577-nm excitation this ratio increases to $29 \%$. When accounting for the fact that the oscillator strength of the Chls in WL-PSII is only about $60 \%$ of that of the initially excited bilins ${ }^{[4,5]}$, it follows that at least $50 \%$ of the initial WL-PBS excitations $(577 \mathrm{~nm}$ ) are transferred to WL-PSII, which is similar to what found in Synechocystis ${ }^{[6]}$. This finding confirms that PBS increase the antenna size of PSII relative to PSI ${ }^{[7,8]}$. 

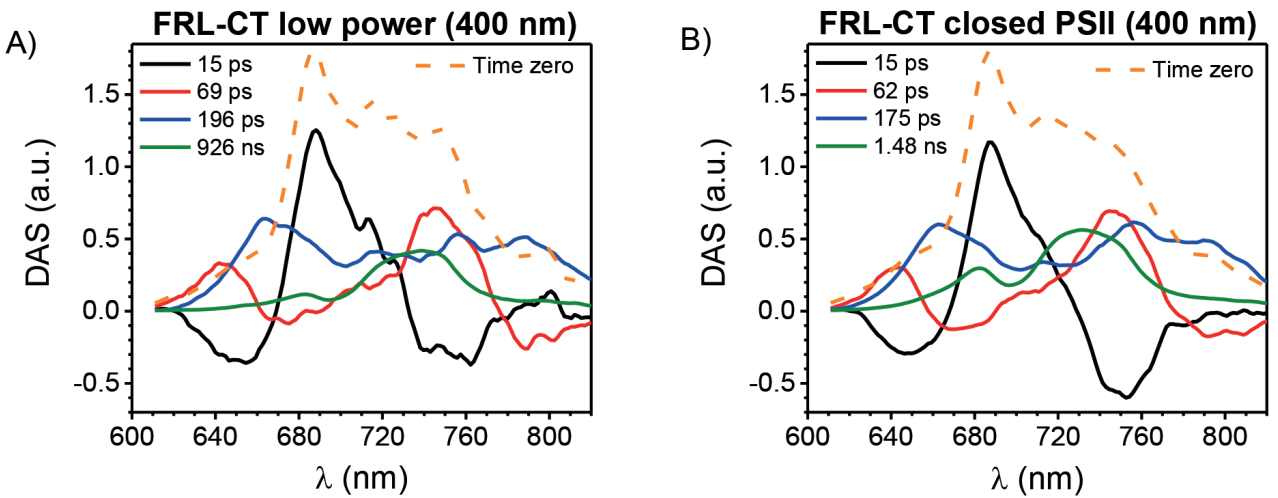

Supplementary Figure 2. TRF measurements of FRL-acclimated cells of CT excited at $400 \mathrm{~nm}$. DAS from TRF measurements on FRL-cells excited at $400 \mathrm{~nm}$ with (nearly) open (A) and closed (B) PSII RCs. The time-zero spectrum (orange dashed lines) is calculated as the sum of all other DAS. The DAS are normalized to the area of the time-zero spectrum for better comparison.

The first two components mostly describe downhill energy transfer from $\mathrm{Chl} a$ to $\mathrm{Chl} f$ (in both FRL-PSI and FRL-PSII, black DAS) and from the majority of Chls $f$ to strongly redshifted Chls $f$ (in FRL-PSI only, red DAS), respectively. The 400-nm laser also excites the PBPs to some extent, as witnessed by the downhill energy transfer features observed in both DASs at shorter wavelengths (similar features dominate the early kinetics when exciting the phycobilisomes prevalently, see Figure 3). A third component, with a lifetime $<200$ ps (blue lines), can be largely assigned to FRL-PSI trapping (in the $750-800 \mathrm{~nm}$ region) and WL-PBS/WL-PSII decay (in the $640-690 \mathrm{~nm}$ region). Some contribution from FRL-BCs and FRL-PSII is probably present at intermediate wavelengths $(710-740 \mathrm{~nm})$. The overall shape/amplitude of this component is not very sensitive to the state of PSII RCs, though the relative amplitude around $680 \mathrm{~nm}$ decreases in closed state in favor of the longer-lived components (cfr blue and green lines in (A) and (B)), confirming the contribution from WLPSIl at these wavelengths. The long-lived DAS (green lines) increases in amplitude and lifetime (from less than $1 \mathrm{~ns}$ in open state to almost $1.5 \mathrm{~ns}$ in closed state) when PSII RCs close and can be mostly ascribed to WL-PSII (with a peak at $680 \mathrm{~nm}$ ) and FRL-PSII (with a peak at 735-740 nm). As we showed in our previous work ${ }^{[1]}$, the spectrum of FRL-PSII in vivo is enriched at shorter wavelengths in comparison to isolated FRL-PSII in vitro due to the contribution of FRL-BCs, advocating for an association between these two units in the cells. This substantial emission by FRL-BCs after 400-nm excitation might result from their direct excitation and, possibly, from a partial equilibration of the excitations formed in FRL-PSII with the redshifted APC of FRL-BCs. Energy transfer from FRL-PSII to FRL-BCs is probably more favored than that from WL-PSII to the WL-PBS core, for two reasons: (i) an entropic factor due to the small number of redshifted Chls in FRL-PSII and (ii) a kinetic factor, as the excitations in FRL-PSII are trapped more slowly than in WL-PSII and have more time to equilibrate with the nearby APC. The ratio between the area of the long-lived 
PSII-related DAS in closed state (Figure 3B, green line) and that of the time zero spectrum (orange dashed line) is about 0.28 for FRL-cells, which is almost two times that observed in WL-cells (0.15). This suggests that FRL-cells have more PSII relative to PSI in comparison to WL-cells.
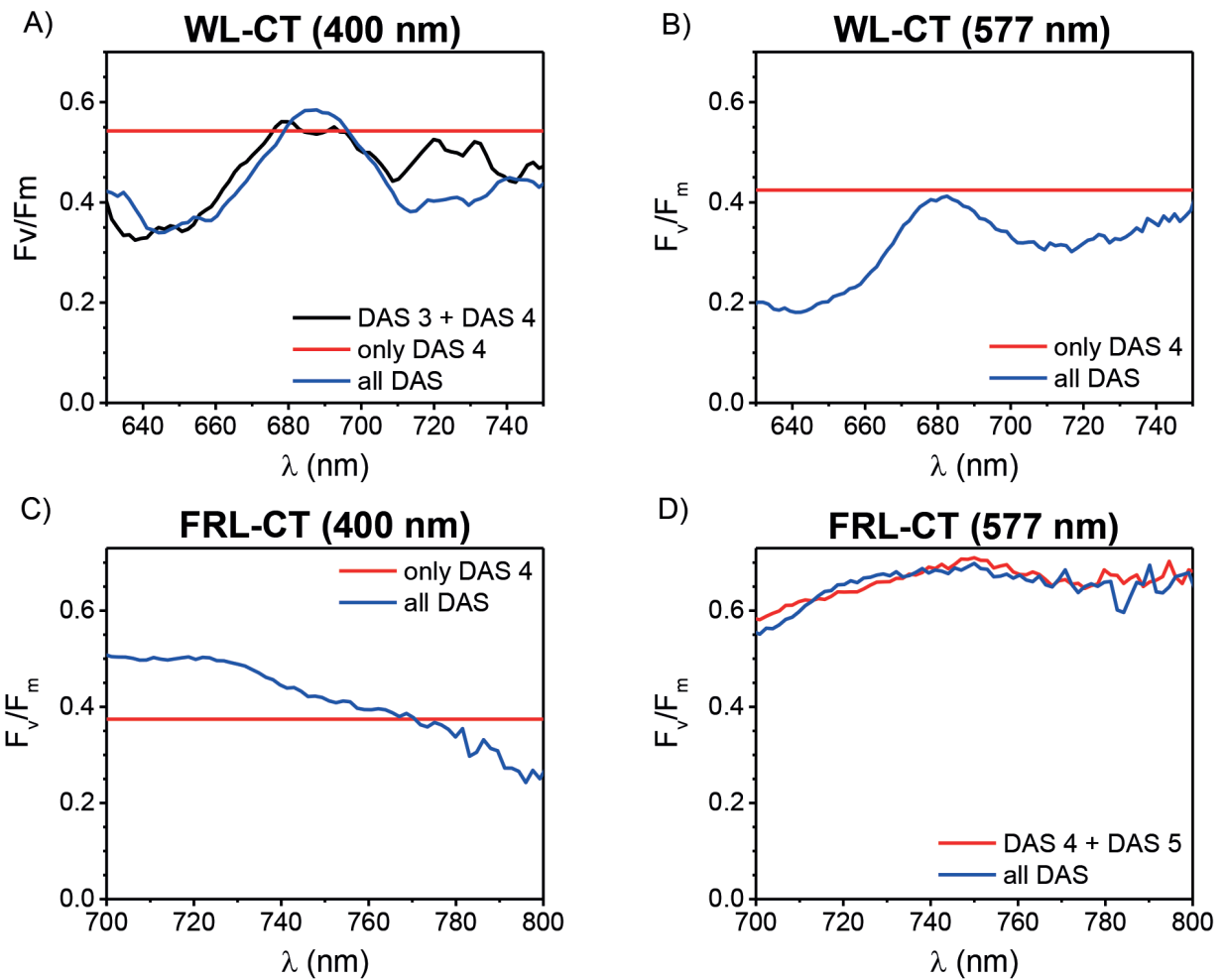

Supplementary Figure 3. PSIl photochemical efficiency of CT cells. $F / F_{m}$ of WL-cells of $C T$ at different wavelengths calculated with different methods from the DAS of TRF measurements (Figures 2, 3, S1, and S2). The aim of these calculations is to evaluate the impact of PSI fluorescence on the estimation of the PSIl efficiency. For this purpose, we compared the $F_{V} / F_{m}$ obtained from the whole TRF data (i.e. using all the DAS, as in Figure 5) to that obtained based on the longest-lived components only, as they are mostly contributed by PSII (and, possibly, the PBPs). Note that both methods are approximations, as in the first case the contribution of PSI is also included in the estimation of the variable fluorescence (which is, in principle, a property of PSII only), whereas in the latter case, the contribution of PSII to the shortest-lived DAS (which is generally not zero) is neglected. In the following, the wavelength-dependent average lifetime is obtained from the DAS as: $\boldsymbol{\tau} \boldsymbol{a v g}(\lambda)=\left(\boldsymbol{\Sigma}_{\boldsymbol{k}} \boldsymbol{D A S} \boldsymbol{S}_{\boldsymbol{k}}(\lambda) \cdot \boldsymbol{\tau}_{\boldsymbol{k}}\right) / \boldsymbol{\Sigma}_{\boldsymbol{k}} \boldsymbol{D A S _ { k }}(\lambda)$, where $\mathrm{k}$ designates the DAS that participate in the average.

A,B) WL-CT upon different excitations: in (A), the black line shows $F_{v} / F_{m}$ obtained as $(1-$ $\tau_{o} / \tau_{m}$ ), where $\tau_{o}$ and $\tau_{m}$ are the average lifetimes in open and closed state, respectively, calculated using only the DAS 3 and 4 of Figure S1 (those with the longest lifetime), which do not contain contributions from PSI (both in the open and in the closed state). The red line displays $1-\tau_{o} / \tau_{m}$, where only the longest lifetime has been used, for both the open and the closed state (as the longest-lived DAS mostly contains contributions from PSII). The blue line is obtained by using all DAS from both measurements (identical to what 
shown in Figure 5C). The data in (B) are obtained in a similar way from the DAS in Figure 2, using only the longest-lived DAS (red line), which are mostly contributed by PSII, and all DAS (blue lines), as also shown in Figure 5C of the manuscript.

$C, D) F_{v} / F_{m}$ of $F R L$-cells of $C T$ at different wavelengths calculated with different methods from the DAS of TRF measurements (Figures S2 and 3). In (C), the red line is obtained as in the previous plots, using only the lifetimes of the longest-lived DAS of Figure S2 in open and closed state (which are mostly contributed by FRL-PSIl; note that FRL-PSII also contributes to the shortest-lived DAS to some extent), while the blue line is obtained using all DAS (as in Figure 5F). In (D), the red line is obtained from the average lifetimes in open and closed state calculated from the longest-lived DAS in open state (Figure 3A) and the two longest-lived DAS in closed state (Figure 3B). The blue line shows $F_{v} / F_{m}$ calculated from all DAS as in Figure 5F.

A)

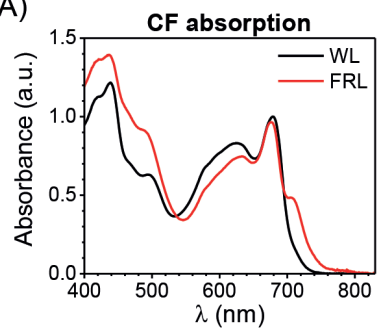

B)

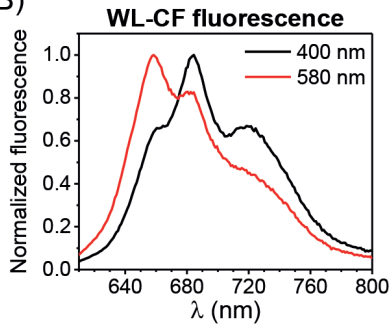

C)

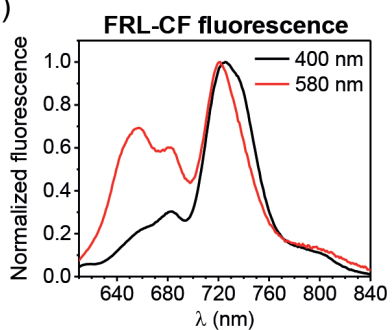

Supplementary Figure 4. Steady-state spectroscopy of intact cells of CF acclimated to WL or FRL. A) Absorption spectra of WL- and FRL-cells (normalized to the area in the region above $580 \mathrm{~nm}$ ) and FRL-WL difference absorption spectrum. B) Florescence spectra of WL-cells excited at 400 and $580 \mathrm{~nm}$. C) Fluorescence spectra of FRL-cells excited at 400 and $580 \mathrm{~nm}$. In comparison to FRL-CT, FRL-CF retains a substantially smaller amount of WL-PSII (compare the emission at $680 \mathrm{~nm}$ in (C) and in Figure 1C of the manuscript). 
A)

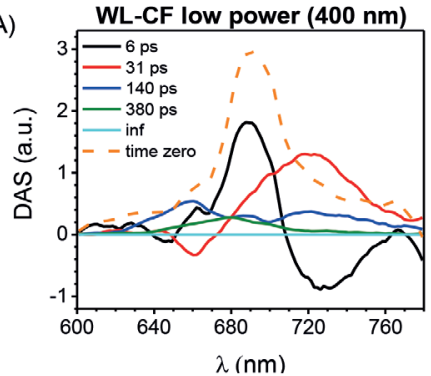

C)

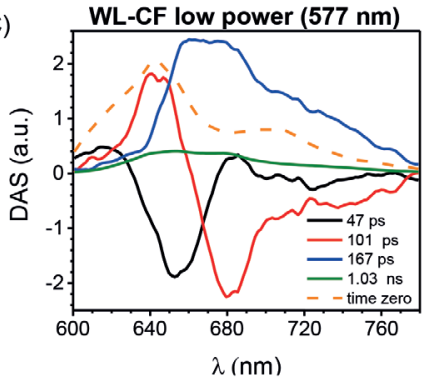

B)

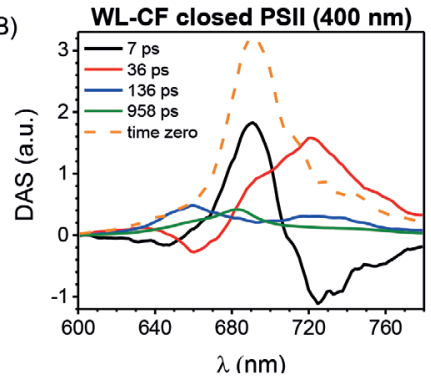

D)

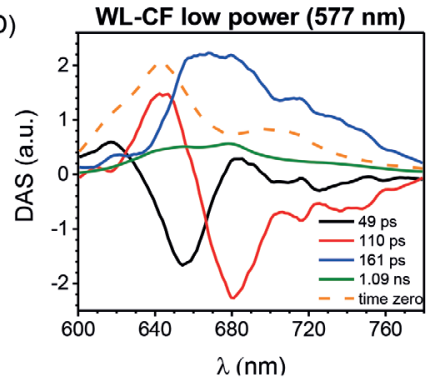

Supplementary Figure 5. TRF measurements of WL-acclimated cells of CF. A,B) DAS from TRF measurements on WL-cells excited at $400 \mathrm{~nm}$ with (nearly) open (A) and closed (B) PSII RCs (as in Mascoli et al.) ${ }^{[1]}$. Note that for the measurement in closed state (B), the DAS were obtained with the lifetime of the first component fixed to a lower value ( $7 \mathrm{ps}$ ) than in our previous work, where all lifetimes where free (with the dataset analyzed being the same). This fixed lower lifetime was needed to obtain a time zero spectrum similar to that obtained in the measurement in open state (A), which is required here for a proper comparison between the two experiments. This is particularly relevant for normalizing the DAS and directly compare the time-integrated steady-state spectra $F_{0}$ and $F_{m}$. This change in the first lifetime did not affect the fitting quality substantially, as the RMS error only increased by $1 \%$. C,D) DAS from TRF measurements on WL-cells excited at $577 \mathrm{~nm}$ with (nearly) open (A) and closed (B) PSII RCs. The small long-lived (inf) component shown in (A) reflects some background signal. For each experiment, the time-zero spectrum (orange dashed lines) is calculated as the sum of all other DAS. The DAS are always normalized to the area of the time-zero spectrum for better comparison.

These results are qualitatively similar to those presented in Figures $\mathrm{S} 1$ and 2 for WL-CT. The main difference is that in WL-CF there is a larger amount of unconnected PBPs/PBS. This is evident from the longest-lived DAS in open state $(C)$, which has a peak at $650 \mathrm{~nm}$ and a lifetime of about $1 \mathrm{~ns}$, indicating that this component is mostly contributed by long-lived PBPs (possibly disconnected PC) rather than open PSII (whose spectrum and lifetime can be obtained from the green DAS in (A)). Based on the area of the green DAS in $(C)$ and that of the time-zero spectrum, it can be estimated that about $15 \%$ of the initially excited PBPs are functionally unconnected to the photosystems and emit at $650 \mathrm{~nm}$. In comparison to the green DAS in (C), the longer-lived (green) DAS in closed state after $577 \mathrm{~nm}$ excitation (D) is enriched in the $680 \mathrm{~nm}$ region, indicating an increased contribution from closed PSII to this component. The presence of a substantial amount of unconnected PBPs is such that the $F_{v} / F_{m}$ after $577 \mathrm{~nm}$ excitation is sizably lower in WL-CF than in WL-CT (cfr. Figure 5 and Figure S8). 

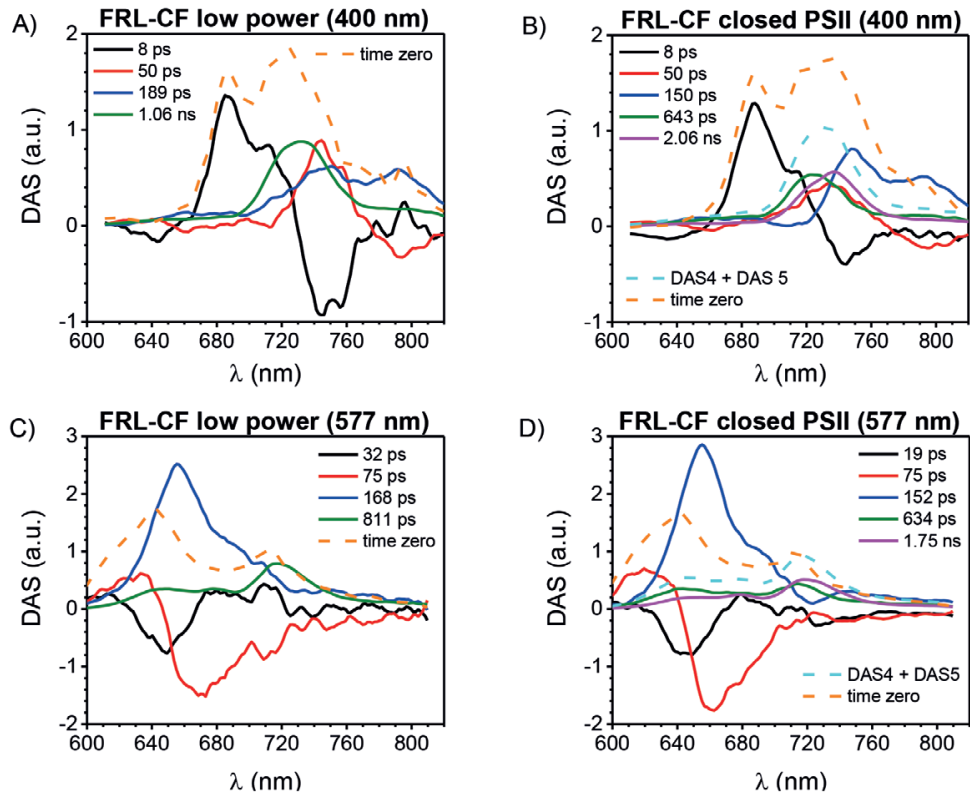

Supplementary Figure 6. TRF measurements of FRL-acclimated cells of CF. A,B) DAS from TRF measurements on FRL-cells excited at $400 \mathrm{~nm}$ with (nearly) open (A) and closed (B) PSII RCs. Note that the lifetimes of the first two components in open state black and red DAS in (A)) were fixed to the values fitted for the dataset with closed PSII $\mathrm{RCs}(\mathrm{B})$. This was required for obtaining similar time-zero spectra in (A) and (B) and make the two measurements more easily comparable. The fitting quality of the data in (A) did not change significantly when fixing the first two lifetimes, as the RMS of the error increased by less than $2 \%$ in comparison to the fitting where all lifetimes were free parameters. C,D) DAS from TRF measurements on FRL-cells excited at $577 \mathrm{~nm}$ with (nearly) open (A) and closed (B) PSII RCs. The small long-lived (inf) component shown in (C) accounts for some background signal. For each experiment, the time-zero spectrum (orange dashed lines) is calculated as the sum of all other DAS. The DAS are always normalized to the area of the time-zero spectrum for better comparison.

These results are qualitatively similar to those presented in Figures S2 and 3 for FRL-CT. The main difference is that FRL-CF retains a much lower amount of WL-PSII (compare (A) and (B) here to Figures S2A and S2B in the $680 \mathrm{~nm}$ region). Furthermore, FRL-CF contains substantial amounts of long-lived unconnected PBPs/PBS, similar to those observed in WL-cells (manifested as the short-wavelength side of the green and magenta DAS in (C) and (D). 
A)

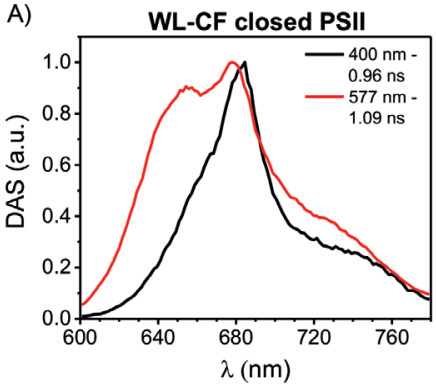

C) FRL-CF: $720 \mathrm{~nm}$ emission

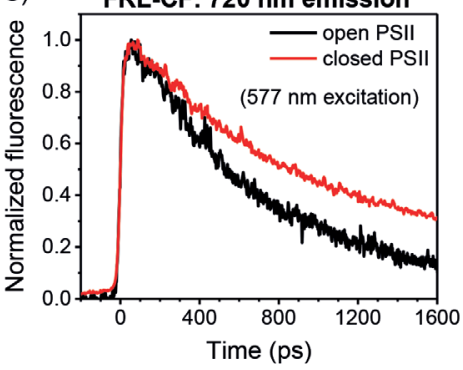

B)

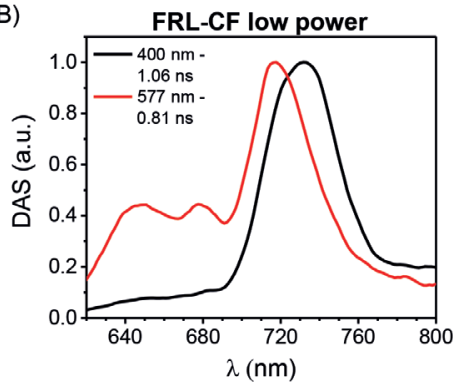

D)

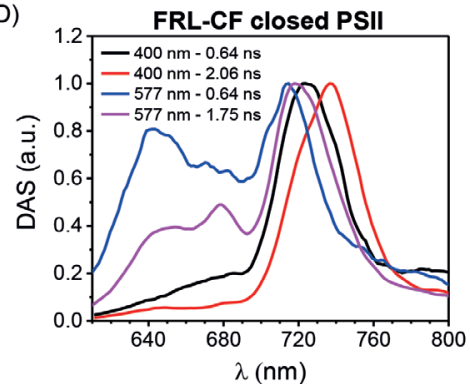

Supplementary Figure 7. TRF measurements of WL- and FRL-acclimated cells of CF. A) Normalized long-lived DAS from TRF measurements of WL-cells with closed PSII RCs after 400 and $577 \mathrm{~nm}$ excitation (corresponding to the green DAS in Figures S5B and S5D). B) Normalized long-lived DAS from TRF measurements of FRL-cells with (nearly) open PSII RCs after 400 and $577 \mathrm{~nm}$ excitation (corresponding to the green DAS in Figures S6A and S6C). C) Normalized fluorescence traces detected at $720 \mathrm{~nm}$ after $577 \mathrm{~nm}$ excitation of FRL-cells with (nearly) open and closed PSII RCs. D) Long-lived DAS (normalized to the peak in the far-red region) from TRF measurements of FRL-cells with closed PSII RCs after 400 and $577 \mathrm{~nm}$ excitation (corresponding to the green and magenta DAS in Figures S6B and S6D).

These results are qualitatively similar to those presented in Figure 4 for CT. One of the main difference, as already pointed out in the captions of the previous figures, is the larger amount of unconnected PBPs found in CF. This is evident, for instance, in the extra shoulder at short wavelengths observed in the 577-nm excited long-lived DAS in WL-CF, which is almost missing after $400 \mathrm{~nm}$ excitation (A). On the other hand, the 400-nm and 577-nm excited long-lived DAS are much more similar in WL-CT (Figure 4A). Similar to FRL-cells of $\mathrm{CT}$, also in FRL-cells of CF the emission at $720 \mathrm{~nm}$ after $577-\mathrm{nm}$ excitation (i.e. emission by FRL-BCs) becomes significantly longer-lived when PSII RCs close (C), i.e. a substantial amount of FRL-BCs are connected to FRL-PSII. The extent of variable fluorescence at 720 $\mathrm{nm}$ is, however, reduced in comparison to FRL-cells of CT (compare (C) to Figure $4 C$ ), suggesting that the connectivity between FRL-BCs and FRL-PSII might be less effective in FRL-CF. In addition, the long-lived far-red fluorescence excited at $577 \mathrm{~nm}$ is blue-shifted in comparison to that excited at $400 \mathrm{~nm}(B, D)$. This points to a larger contribution of FRL-BCs in the far-red fluorescence emitted after 577-nm excitation, consistent with what found for CT. This is also consistent with the observation that the FRL-BCs and FRL-PSII do not fully equilibrate before excited state decay also in FRL-CF. Finally, excited-state decay by FRL-PSII/FRL-BCs in open state is sizably faster after $577 \mathrm{~nm}$ excitation than after $400 \mathrm{~nm}$ 
excitation (B), implying that trapping by FRL-PSII RCs is faster when PBPs (rather than the Chls) are excited. This is also observed in FRL-CT (Figure 4B).

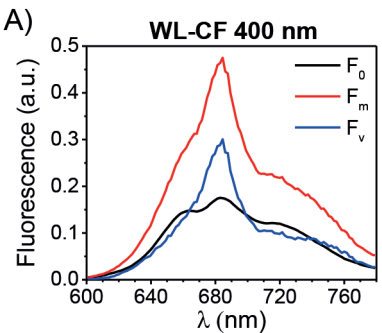

D)

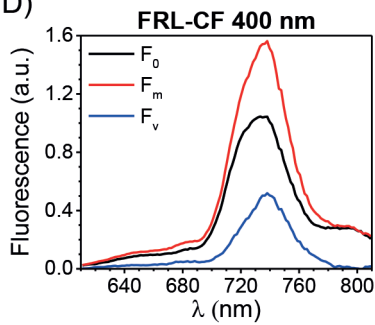

B)

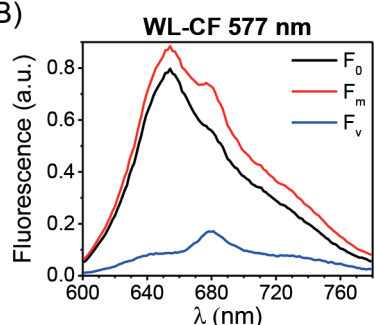

E)

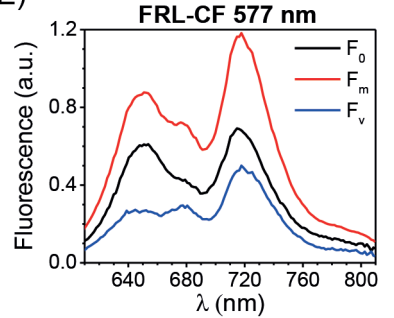

C)

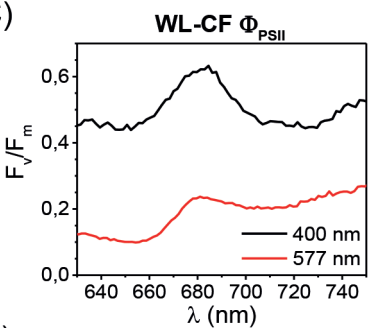

F)

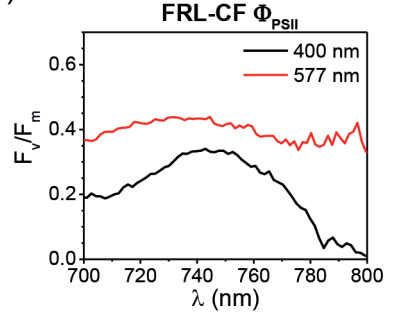

Supplementary Figure 8. Variable fluorescence and PSII photochemical efficiency of WL- and FRL-cells of CF excited at different wavelengths. A,B) Steady-state fluorescence spectra of WL-cells excited at $400 \mathrm{~nm}$ (A) and 577 $\mathrm{nm}(\mathrm{B})$ in open $\left(\mathrm{F}_{\mathrm{o}}\right)$ and closed $\left(\mathrm{F}_{\mathrm{m}}\right)$ state obtained from the DAS in Figure $S 5$. Variable fluorescence spectra $\left(\mathrm{F}_{\mathrm{v}}\right)$ are calculated as the difference between $\mathrm{F}_{\mathrm{m}}$ and $\mathrm{F}_{\mathrm{s}} . \mathrm{C}$ ) $\mathrm{F}_{\mathrm{v}} / \mathrm{F}_{\mathrm{m}}$ of WL-cells at different wavelengths calculated from the spectra in (A) and (B). D,E) Steady-state fluorescence spectra of FRL-cells excited at $400 \mathrm{~nm}(D)$ and $577 \mathrm{~nm}(\mathrm{E})$ in open $\left(F_{o}\right)$ and closed $\left(F_{m}\right)$ state obtained from the DAS in Figure $\left.S 6 . F\right) F_{v} / F_{m}$ of FRL-cells at different wavelengths calculated from the spectra in (D) and (E).

These results are qualitatively similar to those presented in Figure 5 for CT. In the case of the WL-cells, the major difference is the larger amount of unconnected PBPs in CF resulting in a drop in variable fluorescence when the PBPs are excited (compare (B) here and Figure 5B) and, consequently, a much lower $\mathrm{F}_{\mathrm{v}} / \mathrm{F}_{\mathrm{m}}$ after $577 \mathrm{~nm}$ excitation in WL-CF in comparison to WL-CT (compare $(C)$ here and Figure $5 \mathrm{C}$ ).

Panel (F) shows that, similar to FRL-CT, FRL-cells of CF also display higher variable fluorescence after $577 \mathrm{~nm}$ excitation than after $400 \mathrm{~nm}$ excitation (i.e. charge separation by FRL-PSII is more efficient when exciting the PBPs rather than the Chls). This is consistent with trapping by FRL-PSII RCs being faster after PBP excitation than after Chl excitation (as discussed in the caption of Figure S7). In comparison to FRL-CT, the extent of variable fluorescence (and $F_{v} / F_{m}$ ) in FRL-CF is lower in the far-red region, which might be the result of a less effective connectivity between FRL-BCs and FRL-PSII in CF (indeed, after $577 \mathrm{~nm}$ excitation, $F_{v} / F_{m}$ in the $720-740 \mathrm{~nm}$ region is slightly above 0.4 in FRL-CF and nearly 0.7 in FRL-CT). 

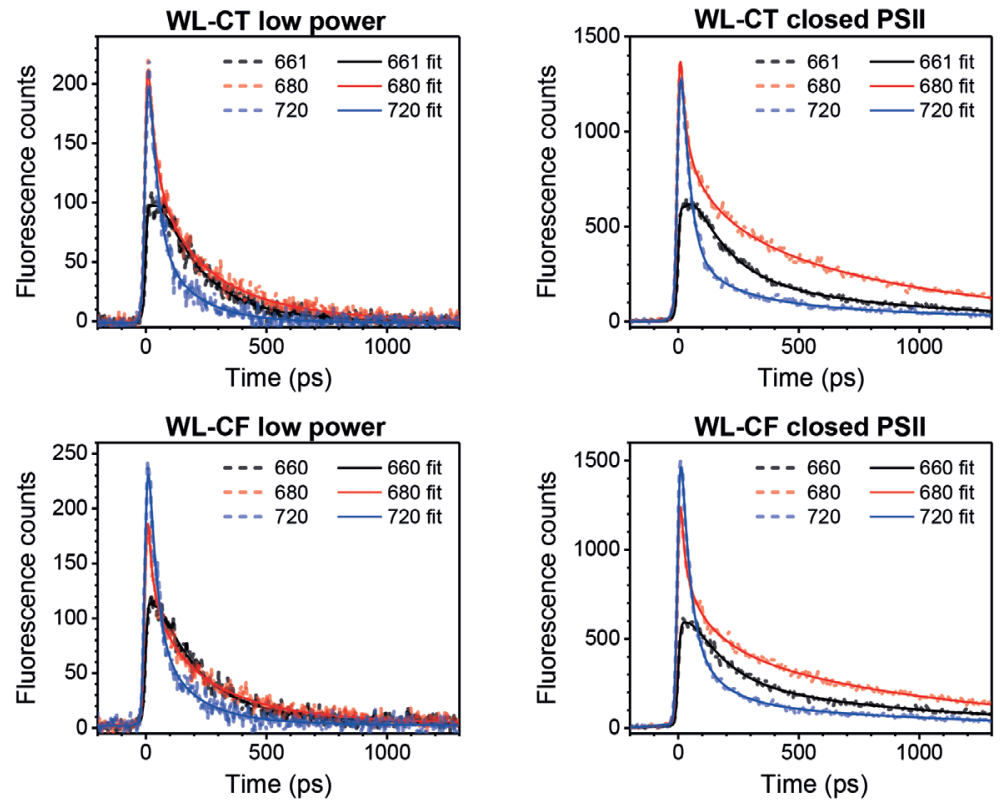

Supplementary Figure 9. TRF measurements of intact cells of WL-acclimated strains excited at $400 \mathrm{~nm}$. Overlay of raw and globally fitted fluorescence time traces excited at $400 \mathrm{~nm}$ at selected emission wavelengths (see Figures S1 and S5 for the DAS from global analysis). The selected emission wavelengths highlight contributions from APC (660 nm), WL-PSII (680 nm), and WL-PSI (720 nm).
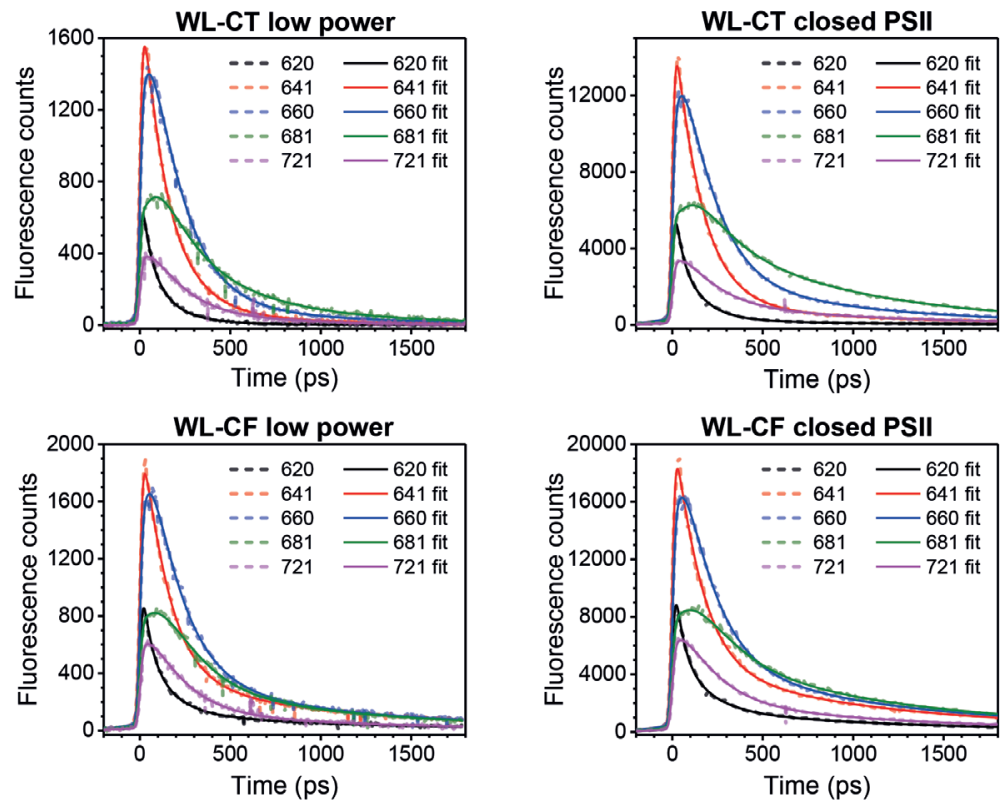

Supplementary Figure 10. TRF measurements of intact cells of WL-acclimated strains excited at $577 \mathrm{~nm}$. Overlay of raw and globally fitted fluorescence time traces excited at $577 \mathrm{~nm}$ at selected emission wavelengths (see Figures 2 and S4 for the DAS from global analysis). The selected emission wavelengths highlight contributions from short-wavelength PC (620 nm), long-wavelength PC (641 nm), APC (660 nm), WL-PSII (681 nm), and WL-PSI $(721 \mathrm{~nm})$. 

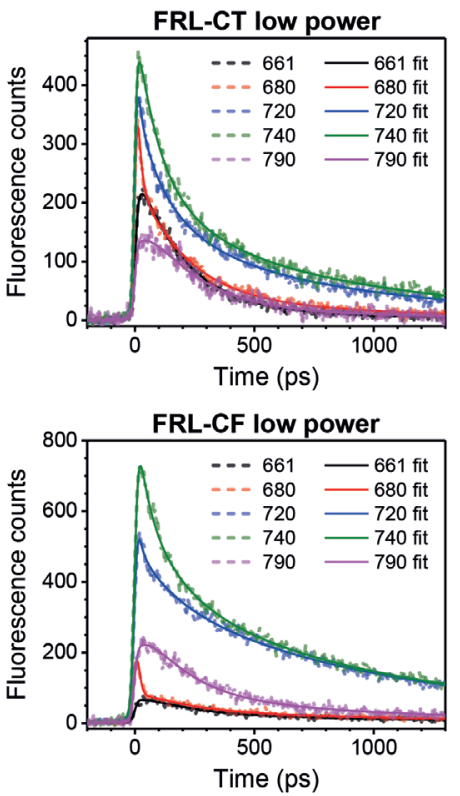

FRL-CT closed PSII

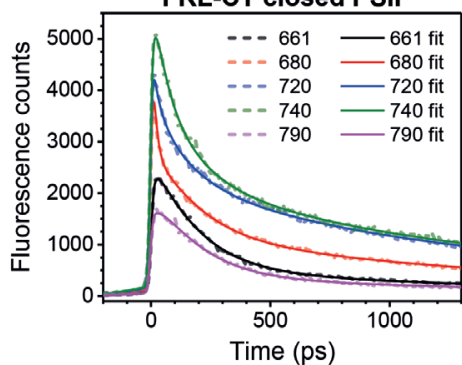

FRL-CF closed PSII

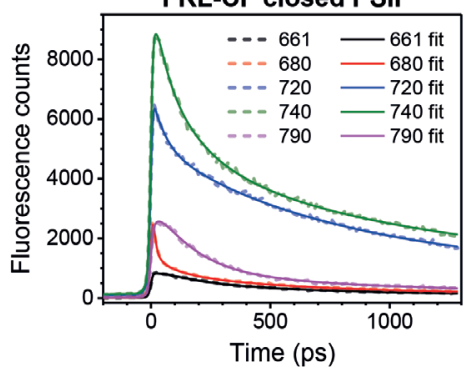

Supplementary Figure 11. TRF measurements of intact cells of FRL-acclimated strains excited at $400 \mathrm{~nm}$. Overlay of raw and globally fitted fluorescence time traces excited at $400 \mathrm{~nm}$ at selected emission wavelengths (see Figures S2 and S6 for the DAS from global analysis). The selected emission wavelengths highlight contributions from APC (660 nm), WL-PSII (681 nm), red-shifted APC (720 nm), FRL-PSII (740 nm), and FRL-PSI (790 nm).
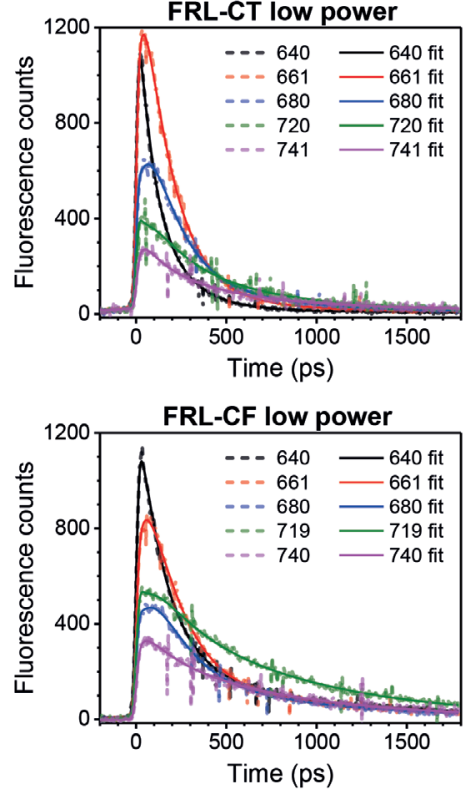

FRL-CT closed PSII
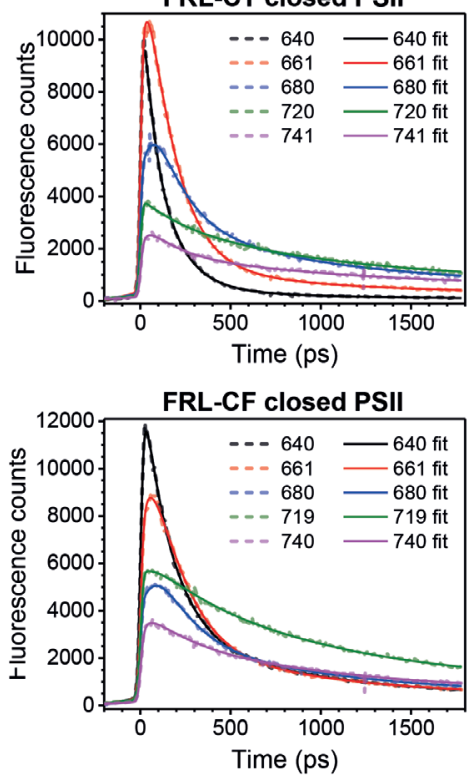

Supplementary Figure 12. TRF measurements of intact cells of FRL-acclimated strains excited at $577 \mathrm{~nm}$. Overlay of raw and globally fitted fluorescence time traces excited at $577 \mathrm{~nm}$ at selected emission wavelengths (see Figures 3 and S5 for the DAS from global analysis). The selected emission wavelengths highlight contributions from PC (640 nm), APC (661 nm), WL-PSII $(680 \mathrm{~nm})$, red-shifted APC (719-720 nm), and FRL-PSII $(740-741 \mathrm{~nm})$. 


\section{CHAPTER}

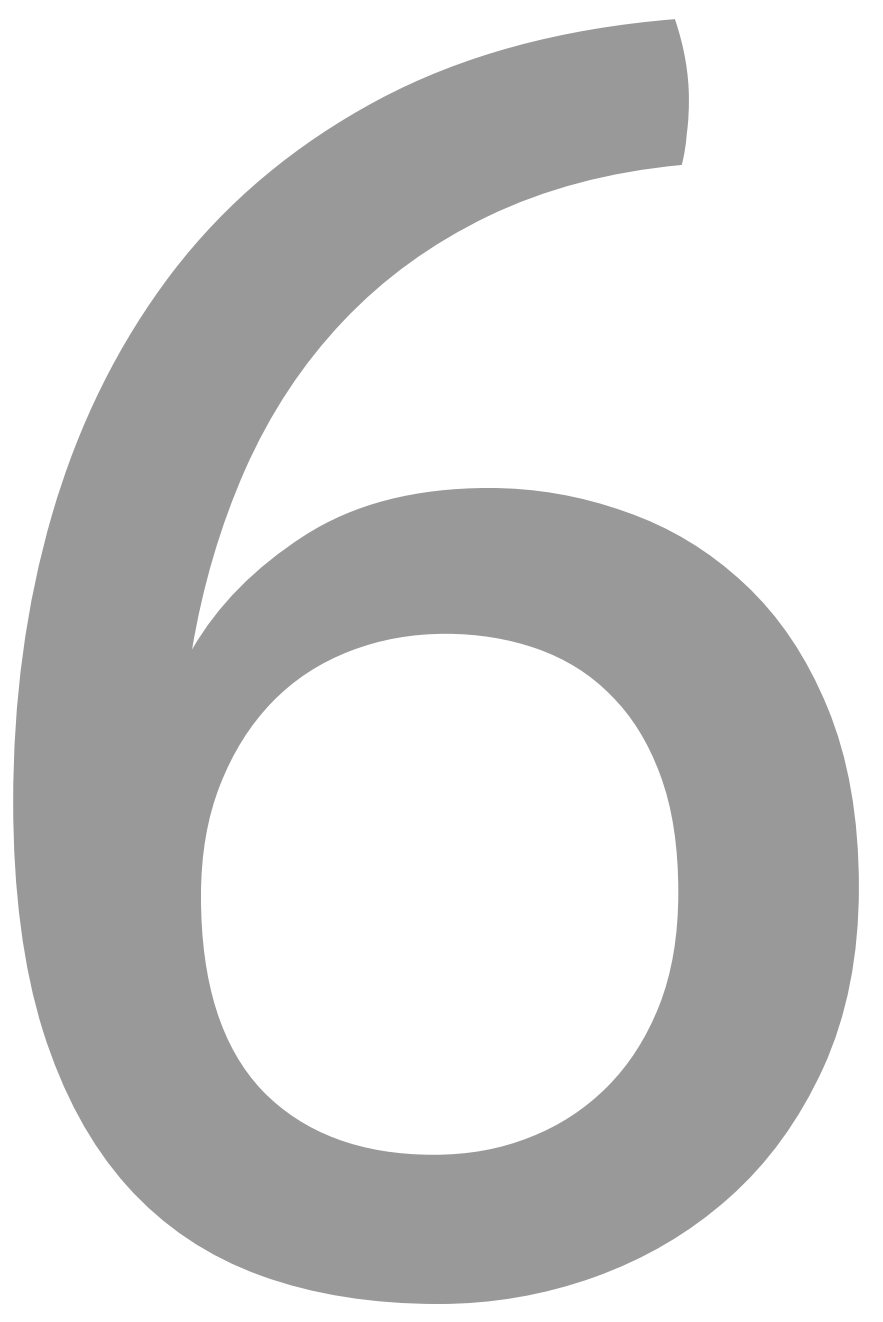


General Discussion 



\section{General Discussion}

Cyanobacteria, living in aquatic habitats, need to regulate their photosynthesis in a constantly changing light environment. They need to harvest enough light for their metabolic needs, while avoiding photodamage. The requirement of sufficient light harvesting at very low light intensities is met by the large amount of photosynthetic pigments embedded in the membrane-bound and membrane-external antennas. However, the changing spectral composition of the light often creates an imbalance between the excitation of PSI and PSII, thereby also creating a redox imbalance between the photochemistry of the two photosystems. State transitions form a mechanism to restore the balance of excitation between PSI and PSII, when the ambient light overexcites one photosystem relative to the other. State transitions in Synechocystis and Synechococcus are studied in chapters 2,3 , and 4 of this thesis.

Excitations created in the pigments of the light-harvesting antenna are eventually transferred to the reaction-center Chls of PSI and PSII. Charge separation in open reaction centers (RCs) is the major de-excitation pathway, handling more than 90 percent ${ }^{[1]}$ of the excitations. Intersystem crossing, thermal energy dissipation and fluorescence also contribute to the excited-state decay. Photochemical RCs of the photosystems can either be in the open state when they are ready for charge separation or in the closed state when charge separation has just occurred. Fluorescence can be used to monitor the state of the RCs. It is minimal when all RCs are open and maximal when all RCs are closed. It has an inverse relation with the other de-excitation pathways, both photochemical and nonphotochemical. Therefore, the study of fluorescence from photosynthetic organisms is a fast, direct and non-destructive method to monitor the light harvesting, photosynthetic performance and, various excitation-energy regulatory processes.

Over the last decades, advances in optical spectroscopy techniques have helped to explore the ultrafast dynamics of light harvesting, (regulation of) excitation-energy transfer (EET) from antenna pigments to the RCs, charge separation and photoprotection. Furthermore, the molecular mechanisms and physical and functional changes underlying the EET regulation mechanism can also be probed with a variety of spectroscopic techniques. Throughout this thesis, picosecond fluorescence spectroscopy is used to investigate the mechanism of state transitions in Synechococcus and Synechocystis and to study the light harvesting and the functional connectivity of the photosynthetic machinery in far-red light acclimated strains of Chlorogloepsis fritschi PCC 6912 (CF) and Chroococcidiopsis thermalis PCC 7203. However, spectroscopy data alone can by no means provide a full picture of the structure and functioning of the photosynthetic apparatus. Spectroscopic methods, in combination with other techniques, such as biochemical and microscopy methods are being used to better understand the structure and functioning of 
photosynthetic organisms from the molecular level up to the cellular level. Fluorescencelifetime-imaging microscopy (FLIM) is used in chapter 4 to study light harvesting, state transitions and distribution of photosystems at the level of individual cells. Previously, FLIM has been used to determine the role of LHCII during state transitions in the green alga Chlamydomonas reinhardtii ${ }^{[2]}$ and a method to study state transition in plant leaves using FLIM was introduced by Wientjes et al ${ }^{[3]}$.

\section{A method to probe the interaction between phycobilisomes and photosystems}

Phycobilisomes (PBSs) are major and very efficient light-harvesting antennas in most cyanobacteria and some red algae. Their diversity and adaptability to different environments is crucial for the success of the cyanobacteria in diverse habitats and environments. Over the last decades, biochemical, biophysical and electron-microscopy techniques have revealed the structure, composition and photophysical properties of PBSs. From these characterizations, various light harvesting and photoprotection strategies in various environments and conditions have been revealed. However, the molecular details about the interaction of PBSs with the thylakoid membrane and photosystems have remained very much in the dark so far. The in vivo characterization of the interaction of PBSs with the membrane and photosystems have been particularly difficult with the biochemical and EM methods. As a working hypothesis, it has been suggested that multiple weak ionic interactions result in the binding of PBSs with the thylakoid membrane and photosystems ${ }^{[4]}$. At low-light intensities, with spectral composition favorable for PBS excitation in comparison with Chl excitation, a transition from state I to state II is induced in cyanobacteria to balance the light harvesting of both photosystems. However, the role of PBSs in the regulation of light harvesting at lowlight intensities (state-transitions conditions) has not been satisfactorily determined yet. We have concluded in chapter 3 that much of the ambiguity about the mechanism of state transitions in cyanobacteria can be traced back to the original ambiguity about the interaction of PBSs with the photosystems. In contrast, in higher plants and green algae, the molecular details of interaction of light-harvesting complexes (LHCII) with photosystems and their phosphorylation/dephosphorylation during state transitions are relatively well understood. To resolve the mechanism of state transitions in cyanobacteria, we aimed at clarifying the role of the PBSs during state transitions. This was done with the use of phosphate buffers. Phosphate buffers are often used to isolate the intact and functional PBSs and to stabilize the interaction of PBSs with the membrane and photosystems. In chapter 3, it is shown that the ultrastructural differences between the PBS-PSII complexes of Synechococcus and Synechocystis are discernible through the comparison of potassiumphosphate-induced fluorescence quenching in both species. By using the same method, also a relative change in the PBS-PSII interaction during state transitions is observed. A hypothesis for the state transitions mechanism presented earlier in chapter 3, was tested to validate the potassium-phosphate-induced quenching method for characterizing the 
PBSs-PSII interaction in different species and the reorganization of the PBS-PSII complex during state transitions. Therefore, the salt-induced-quenching method introduced in chapter 3, appears to be a very simple and useful tool for the in vivo characterization of the ultrastructural differences between the species and possible structural changes in photosynthetic components. With the help of this method we were able to answer/ probe some fundamental questions regarding state transitions in cyanobacteria and provided a different explanation for some data in the literature. It is shown in chapter 3 that the observations such as the presence of a fluorescence decrease in Synechococcus $7942 \triangle A p c D$ and inhibition of fluorescence decrease in Synechocystis $6803 \triangle A p c D$ and Synechococcus $7002 \triangle A p c D$ in state II are artefacts of these mutants. Therefore, these observations do not provide any basis for the state transitions model of McConnell et al [5] which suggests that in a megacomplex of PBS-PSII-PSI, there is increased EET from PBS to PSI in state II besides spillover of EE from PSII to PSI. According to their model, in Synechococcus $7002 \triangle A p c D$ the EET from PBS to PSI is impaired, therefore, in state II, upon PBS excitation the PSII fluorescence decrease does not occur, whereas, spillover of EE from PSII to PSI for Chl a absorbed EE is possible. Note that, prior to the work presented in this thesis, there was only the aforementioned model that attempted to explain the fluorescence changes during state transitions, both in the WT and $\triangle A p C D$ mutant cells upon selective excitation of PBSs and $\mathrm{Chl} a$. The recent proposal that the contribution of PBSs during state transitions is important in Synechocystis but not in Synechococcus [6] is also based on the results of the $\triangle A p c D$ mutants of both species and can be ruled out. Furthermore, it is shown with this method that the PBS mobility does not result in reversible migration of PBSs from PSII to PSI during state transitions as suggested in ${ }^{[7]}$ and concluded in ${ }^{[8,9]}$.

\section{Protein conformational changes in cyanobacteria}

Finding the mechanism of PSII fluorescence quenching in state II upon selective Chl excitation has been of long-standing interest. Since, the apparent purpose of state transitions is to balance the excitation of PSI and PSII, a spillover of EE from PSII to PSI in state II has often been proposed as an intuitive explanation. Nonetheless, the experimental evidence for the spillover of EE from PSII to PSI has never been unambiguously demonstrated. Choubeh et al ${ }^{[10]}$ recently concluded that the PSII quenching in state II occurs without spillover to PSI. In chapter 2 state transitions were characterized in WT and the $\triangle A p c D$ mutant strain of Synechococcus and Synechocystis. Based on these results it was proposed in chapter 3 that the PSII quenching occurs via a conformational change, probably in CP43 or CP47. This hypothesis was confirmed by the kinetics study of state I to state II transition in pure and potassium-phosphate-incubated Synechococcus and Synechocystis (chapter 3). 
Conformational switching of proteins is of a special interest in biology because of its importance for various cellular processes. In photosynthetic organisms, conformational changes in the antenna ${ }^{[11-13]}$ and reaction-center proteins are involved in excitationenergy regulation and electron transfer processes ${ }^{[14]}$. Conformational changes/differences in protein structures can be monitored via changes in the steady-state fluorescence spectra ${ }^{[15,16]}$. The potassium-phosphate-induced fluorescence quenching method as discussed in the previous section, helped us to further confirm and identify the reversible conformational changes in PSII during state transitions. This method might be useful in testing/proposing protein conformational changes in PBSs or in the sub-structures of photosystems.

\section{Thylakoid membrane plasticity and state transitions}

It is concluded in this thesis that in state II a quenched conformation is induced in an increased amount of PSII complexes as compared to state I. It raises the question by what mechanism PSII complexes avoid unnecessary quenching in state I and what induces the quenching in state II? In principle it is possible that the transient disconnection of PBSs from PSII is the immediate cause for the transition from an unquenched to a quenched conformation of PSII. However, it appears to be not very likely because the work of Tian et al ${ }^{[17]}$ showed that there is a significant amplitude of $\sim 1$ ns component (representing unquenched PSII) in the PAL mutant of Synechocystis, which does not contain PBSs. Therefore, most probably, the immediate cause of PSIl quenching is a change in/of the thylakoid membrane in state II. This would require a certain plasticity of the membrane. An earlier study showed that in the Synechocystis 6803 desD/des $A^{-}$mutant, which has more rigid membranes, due to the absence of di- and tri-unsaturated fatty acids ${ }^{[18]}$, state transitions were inhibited at higher temperatures than in WT cells ${ }^{[19]}$. The authors proposed that the inhibition of state transitions in the mutant with modified membrane rigidity are due to the inhibition of the mobility of photosystems in the membrane. However, we have shown in chapter 3 , that both upon PBS and Chl excitation, the quenching of PSIl complexes shows a correlation with PBS mobility instead of mobility of the photosystems. Therefore, we propose that the membrane plasticity required for state transitions in Synechocystis is required to induce the reversible conformational changes in the PSII complexes. Liberton et al ${ }^{[20]}$, investigated the structural changes in thylakoid membranes of Synechocystis upon dark-to-light and light-to-dark transitions. There is a strong correlation between the kinetics of state transitions (Fig. 1, Chapter 2) and the kinetics of reversible reorganization of thylakoid membranes during dark-to-light or light-to-dark transition in Synechcysis cells. Both, the transition from state I to state II (chapter 2) and increase in inter-thylakoid distance are fully completed in $\sim 20$ minutes. Similarly, both the transition from state II to state $I$ and a decrease in inter-thylakoid distance are completed in $\sim 10$ minutes. State transitions are triggered by the redox imbalance in the electron transfer chain between the two photosystems. It was indicated that the structural plasticity in photosynthetic 
membranes of Synechocystis is tied to the function of the photosynthetic electron transfer chain ${ }^{[20]}$. Li et al ${ }^{[8]}$, showed that the fluorescence changes during state transitions are at least partially regulated by changes in the proton concentration on the cytosol side of the thylakoids. The probable origin of the membrane reorganization was also ascribed to variations of surface charges during dark-light transitions ${ }^{[21]}$. These parallels between state transitions and structural changes during dark-to-light/light-to-dark transitions suggest that the thylakoid membranes are involved in the state transitions mechanism. Although small-angle-neutron-scattering (SANS) experiments revealed only the reversible changes in the inter-thylakoid distances ${ }^{[20,21]}$ that were suggested to regulate the PBS interaction with the membrane and the photosystems, it cannot be ruled out that much more nuanced intra-thylakoid changes also accompany state transitions. Reorganization of thylakoid membranes in plants during state transitions have been reported before ${ }^{[22-24]}$. In the work presented in chapter 4, we investigated whether any aggregation effect of ultrastructural changes in membranes during state transitions is observable in FLIM measurements on Synechococcus. However, our results in chapter 4 are unable to reveal any changes in thylakoid membranes related to state transitions. Therefore, this matter still requires further investigation.

\section{PSII kinetics}

Different values for the PSII fluorescence decay times have been reported in previous studies. In chapter 2, two components, with lifetimes $\sim 0.2 \mathrm{~ns}$ and $\sim 0.8-1.0 \mathrm{~ns}$ were resolved for PSII with closed reaction centres. Similar components were resolved before in a PAL mutant of Synechocystis which lacks PBSs ${ }^{[17]}$. The observed fluorescence kinetics were described by the earlier proposed in vitro trap-limited and transfer-to-trap limited models ${ }^{[25,26]}$. However, characterization of state transitions in Synechococcus and Synechocystis showed that only the amplitude of the $\sim 0.2$ ns component increases in state II, (whereas the lifetime of this component remains similar) at the expense of the amplitude of $\sim 0.8 \mathrm{~ns}$ 1.0 ns component (chapter 2). An increase in the fluorescence amplitude can be ascribed to an increase of the population of quenched PSII complexes in state II as compared to state I. In chapter 4 it is concluded that the $\sim 0.2 \mathrm{~ns}$ component represents a quenched population of PSII complexes in both state I and state II.

\section{State transitions and NPQ}

The apparent physiological significance of state transitions is to restore the redox balance in the photosynthetic electron transfer chain for optimal photosynthesis. Therefore, it is intriguing that the magnitude of state transitions in Synechococcus (Synechococcus 7942) is much larger than in Synechocystis (Synechocystis 6803). It was also observed in previous studies ${ }^{[5,27]}$ that state transitions are much more pronounced in Synechococcus $6301^{[27]}$ than in Synechococcus $7002{ }^{[5]}$. On the other hand, orange carotenoid protein (OCP) induced quenching $\left(\mathrm{OCP}_{\mathrm{npq}}\right)$ of PBSs is present in Synechocystis 6803 and Synechococcus 
7002 but not in Synechococcus 7942 and Synechococcus 6301. Therefore, it is very intriguing that for whatever reason, in Synechococcus 7942 and Synechococcus 6301 the OCP-induced quenching of PBSs is not required/present while the extent of quenching via state transition is far higher. Based on the mechanism of state transitions presented in chapter 3, the following explanation for the smaller magnitude of state transitions in Synechocystis is proposed: There are two types of PBSs in Synechocystis regarding their interaction with PSII. The first type has a transient interaction with PSII and therefore the reversible quenching in a fraction of PSII complexes occurs during state transitions. The second type of PBSs shows a stronger/permanent bonding to PSII and consequently that fraction of PBSs and PSII complexes does not participate in state transitions. It has been previously reported that in the mesophilic red alga P. cruentum both PBS mobility and fluorescence changes attributed to state transitions were observed, whereas they were absent in the extremophilic C. caldarium ${ }^{[28]}$. The immobility of PBSs in C. caldarium was attributed to the strong PBS-photosystems interaction. The process of state transitions in C. caldarium was suggested to be replaced by NPQ. However, it is difficult to suggest that in Synechocystis state transitions are partially replaced by $\mathrm{OCP}_{\mathrm{npq}}$, because OCP-induced quenching is activated by high-intensity blue/green light, whereas state transitions are triggered by redox changes in the PQ pool and PSII quenching in state II can be observed in dark-adapted cells. Furthermore, $\mathrm{OCP}_{\mathrm{npq}}$ in Synechocystis occurs in the PBSs, whereas quenching of PSII in state II can be observed upon selective excitation of the Chls. It appears that OCP-induced NPQ and state transitions do not replace each other in cyanobacteria but they might both provide photoprotection in case of overexcitation of PSII. The give and take between $\mathrm{OCP}_{\mathrm{npq}}$ and state transitions is perhaps due to the sharing of PBSs for both mechanisms. While a transient interaction of PBSs and photosystems is required for state transitions it is not certain that $\mathrm{OCP}_{\mathrm{npq}}$ is more/less efficient with stable/mobile PBSs. To disentangle the mechanisms of OCP-induced NPQ and state transitions it appears important to elucidate the structure and function of PBSs for the different species.

\section{Photosynthesis in far-red light}

In oxygenic photosynthesis, due to the energetic requirements of the PSI and PSII photochemistry red light $(680-700 \mathrm{~nm})$ was accepted as an energetic lower limit to drive photosynthesis ${ }^{[29]}$. This was until the discovery of cyanobacterium Acaryochloris in which $97 \%$ of $\mathrm{Chl}$ a is replaced by $\mathrm{Chl} \mathrm{d}$ which absorbs at longer wavelengths than $\mathrm{Chl} a^{[30]}$, even up to $40 \mathrm{~nm}$ more. The view of the "red limit" was completely changed by the discovery that a cyanobacterium Chroococcidiopsis thermalis employs the longest known absorbing pigment Chl f (absorbing up to $800 \mathrm{~nm}$ ) not only for light harvesting but also for CS in the RC of FaRLiP photosystems ${ }^{[30]}$. In FaRLip cyanobacteria, $\mathrm{Chl}$ a is still the dominant pigment comprising $\sim 90 \%$ of total antenna pigments, whereas the content of $\mathrm{Chl} \mathrm{f}$ and $\mathrm{Chl} \mathrm{d}$ is $\sim 10 \%$ and $\sim 1 \%$, respectively ${ }^{[30,31]}$. However, despite the low content of $\mathrm{Chl} \mathrm{f}$, light harvesting above $700 \mathrm{~nm}$ is substantially increased, such that FaRLip cyanobacteria 
can grow in far-red-light rich environment ${ }^{[32]}$, where other species of cyanobacteria, for instance, Synechococcus and Synechocystis cannot survive. However, the insertion of Chl $f$ significantly decreases the photochemical efficiency of PSII ${ }^{[32]}$. The reason for this is proposed to be, i) poor connectivity between the small amount of $\mathrm{Chl} f$ pigments and, ii) slow CS powered by $\mathrm{Chl} f$ in the RC ${ }^{[32]}$. Probably to avoid the low photosynthetic yield when FaRLip cyanobacteria are acclimated to WL, the FarLip photosynthetic machinery is not maintained anymore. In view of the trade-off between light harvesting and light use efficiency in $\mathrm{Chl} f$ containing photosystems ${ }^{[32]}$, the significance of inserting $\mathrm{Chl} f$ for increased crop productivity has yet to be properly investigated. Due to the fact that the discovery of FarLip strains of cyanobacteria was only very recent, the biophysical and structural studies of these systems are very limited so far. As the characterization of FaRLip strains is further extended, the understanding will possibly grow for engineering the high productivity FaRLip systems. For instance the work presented in chapter 5 argues that far-red PBSs have better energetic coupling with the FaRLip PSII reaction centres as compared with the pool of $\mathrm{Chl} f$ embedded in the FaRLip PSII antenna. Consequently, the photochemical efficiency of FaRLip PSII is increased due the incorporation of far-red allophycocyanin in FaRLip Chroococcidiopsis thermalis PCC 7203. 


\section{References}

1. Wientjes, E., H. van Amerongen, and R. Croce, Quantum Yield of Charge Separation in Photosystem II: Functional Effect of Changes in the Antenna Size upon Light Acclimation. The Journal of Physical Chemistry B, 2013. 117(38): p. 11200-11208.

2. Ünlü, C., et al., State transitions in Chlamydomonas reinhardtii strongly modulate the functional size of photosystem II but not of photosystem I. Proceedings of the National Academy of Sciences of the United States of America, 2014. 111(9): p. 3460-3465.

3. Wientjes, E., et al., Imaging the Photosystem I/Photosystem II chlorophyll ratio inside the leaf. Biochimica et Biophysica Acta (BBA) - Bioenergetics, 2017. 1858(3): p. 259-265.

4. Mullineaux, C.W., Phycobilisome-reaction centre interaction in cyanobacteria. Photosynthesis Research, 2007. 95(2): p. 175.

5. McConnell, M.D., et al., Regulation of the distribution of chlorophyll and phycobilin-absorbed excitation energy in cyanobacteria. A structure-based model for the light state transition. Plant physiology, 2002. 130(3): p. 1201-1212.

6. Calzadilla, P.l., et al., Different roles for ApCD and ApcF in Synechococcus elongatus and Synechocystis sp. PCC 6803 phycobilisomes. Biochimica et Biophysica Acta (BBA) - Bioenergetics, 2019. 1860(6): p. 488-498.

7. Joshua, S. and C.W. Mullineaux, Phycobilisome diffusion is required for light-state transitions in cyanobacteria. Plant physiology, 2004. 135(4): p. 2112-2119.

8. $\mathrm{Li}, \mathrm{H}$. , et al., The state transition mechanism—simply depending on light-on and -off in Spirulina platensis. Biochimica et Biophysica Acta (BBA) - Bioenergetics, 2006. 1757(11): p. 1512-1519.

9. Li, D., et al., Light-induced excitation energy redistribution in Spirulina platensis cells: "spillover" or "mobile PBSs"? Biochimica et Biophysica Acta (BBA) - Bioenergetics, 2004. 1608(2): p. 114-121.

10. Ranjbar Choubeh, R., et al., State transitions in the cyanobacterium Synechococcus elongatus 7942 involve reversible quenching of the photosystem II core. Biochimica et Biophysica Acta (BBA) - Bioenergetics, 2018. 1859(10): p. 1059-1066.

11. Croce, R., J. Breton, and R. Bassi, Conformational Changes Induced by Phosphorylation in the CP29 Subunit of Photosystem II. Biochemistry, 1996. 35(34): p. 11142-11148.

12. Liguori, N., et al., From light-harvesting to photoprotection: structural basis of the dynamic switch of the major antenna complex of plants (LHCII). Scientific Reports, 2015. 5(1): p. 15661.

13. Sunku, K., H.J. de Groot, and A. Pandit, Insights into the photoprotective switch of the major light-harvesting complex II (LHCII): a preserved core of arginine-glutamate interlocked helices complemented by adjustable loops. J Biol Chem, 2013. 288(27): p. 19796-804.

14. Shlyk, $\mathrm{O}$, et al., $A$ single residue controls electron transfer gating in photosynthetic reaction centers. Scientific Reports, 2017. 7(1): p. 44580.

15. van Oort, B., et al., Equilibrium between Quenched and Nonquenched Conformations of the Major Plant Light-Harvesting Complex Studied with High-Pressure Time-Resolved Fluorescence. The Journal of Physical Chemistry B, 2007. 111 (26): p. 7631-7637. 
16. Mátyus, L., J. Szöllősi, and A. Jenei, Steady-state fluorescence quenching applications for studying protein structure and dynamics. Journal of Photochemistry and Photobiology B: Biology, 2006. 83(3): p. 223-236.

17. Tian, L., S. Farooq, and H. van Amerongen, Probing the picosecond kinetics of the photosystem II core complex in vivo. Phys Chem Chem Phys, 2013. 15(9): p. 3146-54.

18. Tasaka, Y., et al., Targeted mutagenesis of acyl-lipid desaturases in Synechocystis: evidence for the important roles of polyunsaturated membrane lipids in growth, respiration and photosynthesis. The EMBO journal, 1996. 15(23): p. 6416-6425.

19. El Bissati, K., et al., Photosystem II fluorescence quenching in the cyanobacterium Synechocystis PCC 6803: involvement of two different mechanisms. Biochimica et Biophysica Acta (BBA) Bioenergetics, 2000. 1457(3): p. 229-242.

20. Liberton, M., et al., Organization and flexibility of cyanobacterial thylakoid membranes examined by neutron scattering. The Journal of biological chemistry, 2013. 288(5): p. 3632-3640.

21. Nagy, G., et al., Reversible membrane reorganizations during photosynthesis in vivo: revealed by small-angle neutron scattering. Biochemical Journal, 2011. 436(2): p. 225-230.

22. Chuartzman, S.G., et al., Thylakoid membrane remodeling during state transitions in Arabidopsis. The Plant cell, 2008. 20(4): p. 1029-1039.

23. Tikkanen, M., et al., Post-genomic insight into thylakoid membrane lateral heterogeneity and redox balance. FEBS Lett, 2012. 586(18): p. 2911-6.

24. Minagawa, J., State transitions-The molecular remodeling of photosynthetic supercomplexes that controls energy flow in the chloroplast. Biochimica et Biophysica Acta (BBA) - Bioenergetics, 2011. 1807(8): p. 897-905.

25. Szczepaniak, M., et al., Charge Separation, Stabilization, and Protein Relaxation in Photosystem II Core Particles with Closed Reaction Center. Biophysical Journal, 2009. 96(2): p. 621-631.

26. van der Weij-de Wit, C.D., et al., Charge Separation is Virtually Irreversible in Photosystem II Core Complexes with Oxidized Primary Quinone Acceptor. The Journal of Physical Chemistry A, 2011. 115(16): p. 3947-3956.

27. Mullineaux, C.W. and J.F. Allen, State 1-State 2 transitions in the cyanobacterium Synechococcus 6301 are controlled by the redox state of electron carriers between Photosystems I and II. Photosynthesis Research, 1990. 23(3): p. 297-311.

28. Kaňa, R., et al., Phycobilisome mobility and its role in regulation of light-harvesting in red algae. Plant physiology, accepted, 2013.

29. Björn, L.O., G.C. Papageorgiou, and R.E. Blankenship, A viewpoint: why chlorophyll a? Photosynthesis research, 2009. 99(2): p. 85-98.

30. Nürnberg, D.J., et al., Photochemistry beyond the red limit in chlorophyllf-containing photosystems. Science, 2018. 360(6394): p. 1210-1213.

31. Chen, M., et al., A cyanobacterium that contains chlorophyll $f$ - a red-absorbing photopigment. FEBS Letters, 2012. 586(19): p. 3249-3254.

32. Mascoli, V., L. Bersanini, and R. Croce, Far-red absorption and light-use efficiency trade-offs in chlorophyll f photosynthesis. Nat Plants, 2020. 6(8): p. 1044-1053. 
CHAPTER

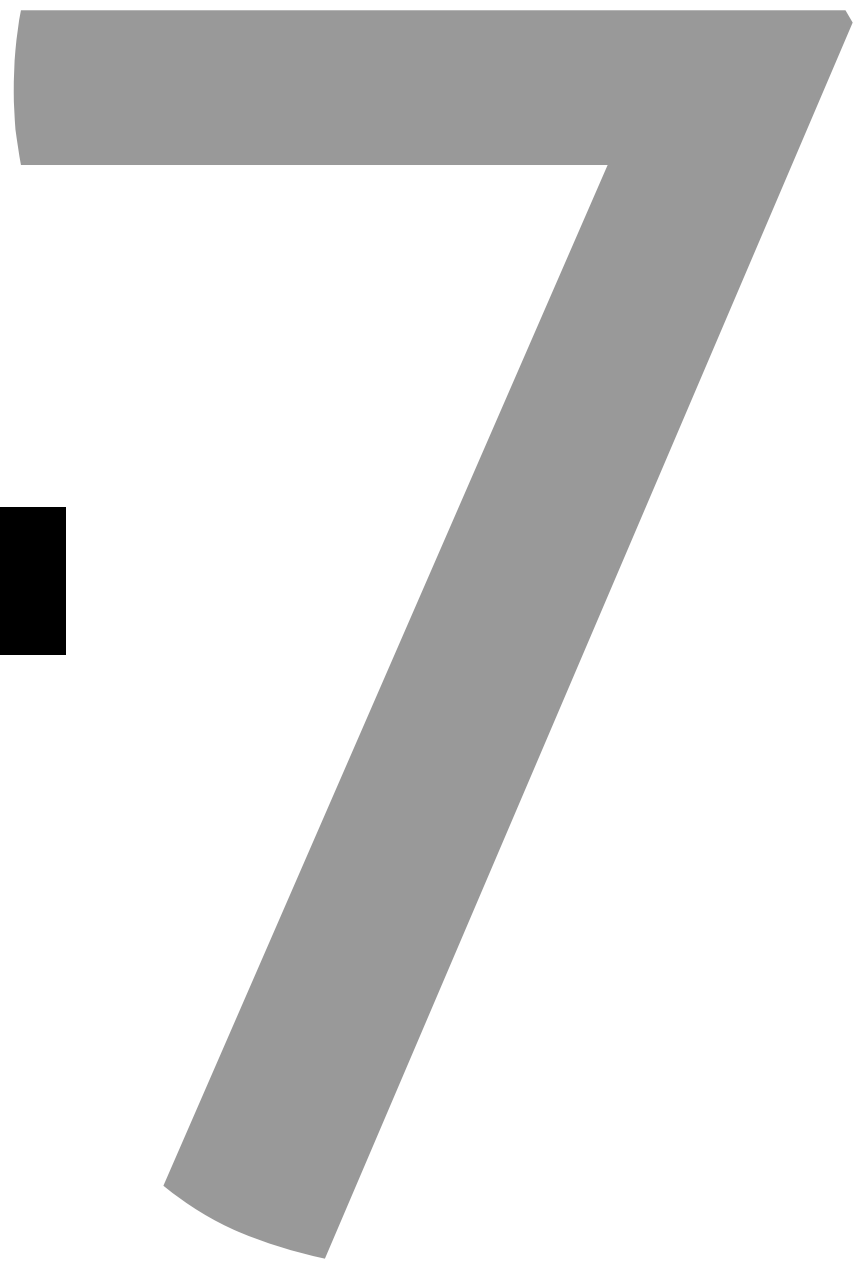


Summary 



\section{Summary}

In oxygenic photosynthetic organisms two photosystems, namely photosystem I (PSI) and photosystem II (PSII) independently harvest the sunlight and drive the primary photochemistry. The two photosystems work in series and for the optimal/maximum photosynthetic efficiency their excitations must be balanced. State transitions form a very important regulatory mechanism in oxygenic photosynthetic organism (green plants, algae, cyanobacteria) which contributes to an optimal photosynthetic performance in constantly changing light conditions. Despite the rigorous research on cyanobacterial state transitions for many decades, the systematic understanding of the process is still elusive. The main purpose of this thesis was to resolve the mechanism and structural basis of phycobilisome (PBS)-absorbed and chlorophyll (Chl) $a$ - absorbed excitation-energy (EE) regulation during state transitions in two different species of cyanobacteria, i.e., Synechococcus and Synechocystis. Consequently, a mechanism for the state transitions in cyanobacteria is determined in this work. Furthermore, a salt-induced-quenching method is introduced to probe the interaction of PBSs with membrane and photosystems in vivo and to characterize the conformational states of PBSs and PSII. Time-resolved fluorescence spectroscopy and fluorescence-lifetime-imaging microscopy were used as main techniques. The main findings from all chapters are summarized below.

In chapter 2 light harvesting, excitation-energy transfer (EET) and state transitions in wildtype (WT), $\triangle \mathrm{ApcD}$ and $\triangle \mathrm{ApcF}$ strains of Synechococcus and Synechocystis were probed at physiological conditions. In the $\triangle \mathrm{ApcF}$ strains EET from PBSs to PSII (or probably both photosystems) was found to be significantly impaired as compared to $\triangle A p c D$ strains. In Synechococcus in both $\triangle \mathrm{ApcD}$ and $\triangle \mathrm{ApcF}$ strains the state transitions were pronounced (slightly stronger than in the WT strain), showing that the impairment of excitationenergy transfer (EET) form PBS to photosystems due to the deletion of $\triangle A p c D$ and $\triangle A p c F$ units does not make the state transitions process inefficient. From the analysis of the timeresolved fluorescence data it was concluded that in cyanobacteria, the light harvesting by PSII remains similar in both state I and state II and the PSII fluorescence decrease in state II can be explained by PSII quenching.

In chapter 3 the physiological basis of PSII quenching and the (non-)involvement of PBSs during state transitions was investigated. It was hypothesized that certain subtle differences in the molecular details of PBSs to PSII interaction between Synechococcus and Synechocystis can explain as to how the fluorescence changes attributed to state transitions are inhibited in Synechocystis $\triangle A p c D$ but not in Synechococcus $\triangle A p c D$. An unstable/ transient interaction between PBS and PSIl was proposed to allow the reconfiguration of PBS-PSII complexes and reversible conformational changes in PSII during state transitions. Reversible ultrastructural changes in thylakoid membranes were also proposed to induce 
reversible conformational and organizational changes in PSII complexes. From the kinetic study of the state I to state II transition in pure and potassium-phosphate-incubated cells of Synechococcus it was demonstrated that at the basis of PSIl quenching is structural flexibility of PSII core antenna complexes. The potassium-phosphate-induced quenching was shown to be very sensitive to the interaction details of PBSs with PSII and the conformational state of the PSII complexes. With the help of the salt-induced-quenching method it was demonstrated that the apparent differences regarding the mechanism of state transitions between Synechococcus and Synechocystis suggested by their $\triangle A p c D$ strains are mere artefacts of these mutants.

In chapter 4 the global distribution of photosystems and state transitions were mapped in individual cells of cyanobacterium Synechococcus. In thylakoid membranes, the microdomains relatively rich either in PSI or PSII were found to be stable when a transition from state I to state II was induced in cells. No large scale (sub-micrometre) changes in the distribution of photosystems were observed during state transitions. The ratio of unquenched PSII/quenched PSII was found nearly homogenous throughout the cells despite an inhomogeneous distribution of photosystems. PSI was concluded to have a higher concentration in the inner thylakoids as compared to the outer ones.

In Chapter 5 the characterization of white-light (WL) and far-red light (FRL) photoacclimated (FaRLip) strains of Chlorogloepsis fritschi (CF) and Chroococcidiopsis thermalis PCC 7203 (CT) has shown that the photosynthetic machinery of the FaRLip strains of these species is very heterogeneous. After several weeks of acclimation to far-red light, FRL-photosystems incorporating red-shifted Chls and FRL-bi-cylinderical cores (FRL-BCs) containing redshifted APC are synthesized. The analysis of the time-resolved fluorescence data shows that the incorporation of far-red APC increases both the absorption cross-section and photochemical efficiency of chlorophyll $f$-containing PSII. 
Summary 

Acknowledgements 

For the completion of this work I am thankful to many people who hepled me in different ways.

Herbert, first and foremost my gratitude to you for initiating me into the interdisciplinary and rich field of photosynthesis and offering the Ph.D. position under your supervision. Your inexhaustible patience, compassion, guidance and encouragement were indeed a great help over the years, particularly when things were not moving forward for a long time. Discussions with you whether brief or lengthy were unfailingly insightful and pleasant. I take those meetings as an important part of my training as a scientist. Interestingly, often it was those short comments, which were apparently made casually, that were the ones which would reveal the fallacy of my arguments and made me rethink the problem. Above all, I found in Herbert a remarkable human being which during the COVID period was confirmed yet again, when he immediately prioritized the safety of lives much above the research output of the lab.

Emilie, I am very thankful to you for being my co-supervisor. You have been always kind, friendly, helpful, appreciative and available for discussions.

Yashar, I cannot thank you enough for teaching me everything, from cell culturing to the use of several instruments at the beginning of my Ph.D. You were also very helpful to me in many other matters regarding my stay in Wageningen. I still cherish the discussions we used to have on a myriad of topics on our way to FORUM for lunch. It was quite fun to go to conferences together. Arjen, none of my experimental works would have had a chance of being successful if it were not for your unwavering help, technical support and assistance. You were always a pleasant company. Cor thank you for your help in growing bacteria and for the lab supplies. I always found you in good humor. John Philippi, thank you for always helping me with technical matters. I would also like to thank Johannes, Frank, Rob, Netty, Brenda, Camilla, Sonja, John, Jeremy, Sam, Cleo, Vincent and Dana for creating a nice atmosphere in BIP. Hildert and Madelief, it was a pleasure working with you during your stay in BIP for the thesis work.

Being part of the SE2B network was a wonderful and invaluable experience. Thank you all ESRs and PIs for the most pleasant environment during the meetings. Christo, thank you, for being a wonderful friend and colleague for all these years. Thank you and Manja for hosting me in Verona and for showing around that cute city. I am particularly grateful for the collaboration work with Prof. Roberta Croce and Vincenzo, without your generous help this thesis would be literally incomplete. I learned a great deal from both of you. Thank you, Vincenzo, for your visits to Wageningen, you were a great company. I would also like to thank Fernando and Diana Kirilovsky, for successful collaborations, for providing the strains of cyanobacteria and for the helpful discussions during the meetings. 
Our Ph.D. room was indeed the most lively and pleasant place in BIP. For this lovely atmosphere, I must thank Abbas, Yashar, Suyeon, Carel, Tatiana, Donny, Koen, Klaudia, Raquel, Wouter, Benjamin, Sasan, Peter, Christo, Ludovico, Lavanya, Mattia and Morwarid. Suyeon it was an absolute pleasure to share the office with you. We did not manage to go to New Zealand and France, but we can consider our trips to the campus plaza for the Doppio coffee a nice substitute for that. Abbas and Ludovico, you brought a lot of positivity and optimism in the office. Klaudia, thank you for being an excellent neighbor both in Plantsoen and in the office. Du, I have many fond memories of you from one year of your stay as my neighbor in the office. Thank you for all those heartening and insightful conversations. I look forward to meeting you again.

Living in Wageningen, gave me a unique opportunity to develop worthy friendships and acquaintances from all over the World. Thank you very much my Plantsoen family for the rich multicultural atmosphere, for excellent dinners and lighthearted chatting. I would particularly like to thank Monica, Simen, Stamatis, Kazim, Bea, Marta, Mirella, Manolis, Caro, Pepe, Catherine, Caixai, Andrea, Amit, Antonio, Alessandro, Alvaro, Lucas, Enrico and Helena, whose presence in Plantsoen made it feel like home. Thank you, Monica and Stamatis, for your constant support and friendship even though you left the Netherlands quite some time ago. The Pakistani community in Wageningen, though small never made me feel homesick. I would particularly like to thank Haris, Munawar and Hasnain Shah for their pleasant company and delicious dinners. I would also like to thank Naeem, Qaisar and Shehzad who always found time to meet me despite living in different countries with busy schedules.

Brenda, many thanks to you for being very helpful in many administrative matters.

I lack words to express my deepest gratitude to my family in Pakistan. Their love, unconditional support, encouragement and advises made it possible for me to live happily and work in foreign countries. 
The research described in this thesis was financially supported by the European Union's Horizon 2020 research and innovation program under grant agreement no. 675006 (SE2B)

Research presented in this thesis was mainly carried out at the Laboratory of Biophysics, Wageningen University \& Research, Wageningen, The Netherlands

Research presented in this thesis was partly carried out at VU, Amsterdam, The Netherlands 